Florida International University

FIU Digital Commons

FIU Electronic Theses and Dissertations

University Graduate School

$11-12-2014$

\title{
Movement Effects on the Flow Physics and Nutrient Delivery in Engineered Valvular Tissues
}

Manuel Salinas

msalinas@nova.edu

Follow this and additional works at: https://digitalcommons.fiu.edu/etd

Part of the Biological Engineering Commons, Biomechanical Engineering Commons, Biomechanics and Biotransport Commons, Biotechnology Commons, and the Other Biomedical Engineering and Bioengineering Commons

\section{Recommended Citation}

Salinas, Manuel, "Movement Effects on the Flow Physics and Nutrient Delivery in Engineered Valvular Tissues" (2014). FIU Electronic Theses and Dissertations. 1924.

https://digitalcommons.fiu.edu/etd/1924

This work is brought to you for free and open access by the University Graduate School at FIU Digital Commons. It has been accepted for inclusion in FIU Electronic Theses and Dissertations by an authorized administrator of FIU Digital Commons. For more information, please contact dcc@fiu.edu. 


\title{
FLORIDA INTERNATIONAL UNIVERSITY
}

Miami, Florida

\section{MOVEMENT EFFECTS ON THE FLOW PHYSICS AND NUTRIENT DELIVERY \\ IN ENGINEERED VALVULAR TISSUES}

\author{
A dissertation submitted in partial fulfillment of \\ the requirements for the degree of \\ DOCTOR OF PHILOSOPHY \\ in \\ BIOMEDICAL ENGINEERING \\ by
}

Manuel Salinas

2015 
To: Dean Amir Mirmiran

College of Engineering and Computing

This dissertation, written by Manuel Salinas, and entitled Movement Effects on the Flow Physics and Nutrient Delivery in Engineered Valvular Tissues, having been approved in respect to style and intellectual content, is referred to you for judgment.

We have read this dissertation and recommend that it be approved.

Vinu Unnikrishnan

Nikolaos Tsoukias

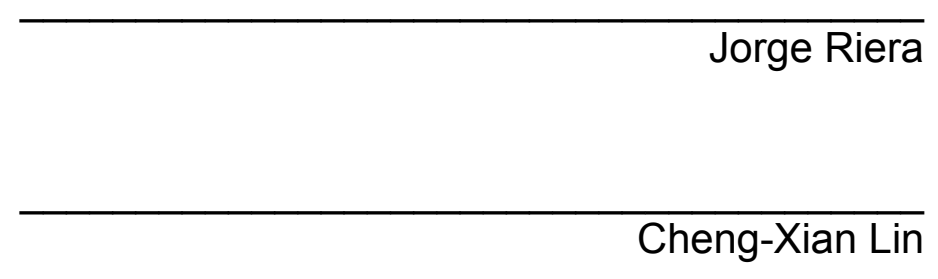

Date of Defense: November 12, 2014

Sharan Ramaswamy, Major Professor

The dissertation of Manuel Salinas is approved.

Dean Amir Mirmiran

Dean of the College of Engineering and computing

Dean Lakshmi N. Reddi

University Graduate School

Florida International University, 2015 


\section{ACKNOWLEDGMENTS}

I wish to thank my advisor Dr. Sharan Ramaswamy for his continuous guidance. I would also like to thank my committee members Dr. Vinu Unnikrishnan, Dr. Nikolaos Tsoukias, Dr. Jorge Riera, and Dr. Cheng-Xian Lin for directing my research in the right direction, the MBRS-RISE program for its generous support, and the BME faculty and Staff. Finally, I wish to thank my family for their patience and support. 


\section{ABSTRACT OF THE DISSERTATION \\ MOVEMENT EFFECTS ON THE FLOW PHYSICS AND NUTRIENT DELIVERY \\ IN ENGINEERED VALVULAR TISSUES}

by

Manuel Salinas

Florida International University, 2015

Miami, Florida

\section{Professor Sharan Ramaswamy, Major Professor}

Mechanical conditioning has been shown to promote tissue formation in a wide variety of tissue engineering efforts. However the underlying mechanisms by which external mechanical stimuli regulate cells and tissues are not known. This is particularly relevant in the area of heart valve tissue engineering (HVTE) owing to the intense hemodynamic environments that surround native valves. Some studies suggest that oscillatory shear stress (OSS) caused by steady flow and scaffold flexure play a critical role in engineered tissue formation derived from bone marrow derived stem cells (BMSCs). In addition, scaffold flexure may enhance nutrient (e.g. oxygen, glucose) transport. In this study, we computationally quantified the i) magnitude of fluid-induced shear stresses; ii) the extent of temporal fluid oscillations in the flow field using the oscillatory shear index (OSI) parameter, and iii) glucose and oxygen mass transport profiles. Noting that sample cyclic flexure induces a high degree of oscillatory shear stress (OSS), we incorporated moving boundary computational fluid dynamic simulations of samples housed within a bioreactor to consider the effects of: 1) 
no flow, no flexure (control group), 2) steady flow-alone, 3) cyclic flexure-alone and 4) combined steady flow and cyclic flexure environments. We also coupled a diffusion and convention mass transport equation to the simulated system. We found that the coexistence of both OSS and appreciable shear stress magnitudes, described by the newly introduced parameter OSI- $\overrightarrow{|\tau|}$, explained the high levels of engineered collagen previously observed from combining cyclic flexure and steady flow states. On the other hand, each of these metrics on its own showed no association. This finding suggests that cyclic flexure and steady flow synergistically promote engineered heart valve tissue production via OSS, so long as the oscillations are accompanied by a critical magnitude of shear stress. In addition, our simulations showed that mass transport of glucose and oxygen is enhanced by sample movement at low sample porosities, but did not play a role in highly porous scaffolds. Preliminary in-house in vitro experiments showed that cell proliferation and phenotype is enhanced in OSI- $\mid \overrightarrow{\tau \mid}$ environments. 
CHAPTER 2: HEART VALVES ANATOMY, DISEASE AND PROSTHETIC DEVICES

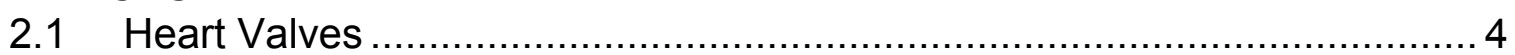

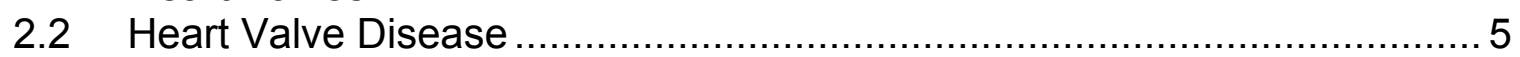

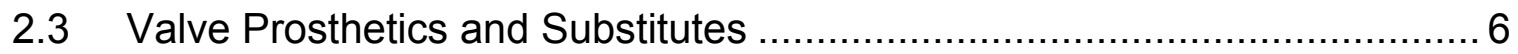

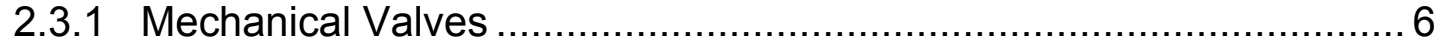

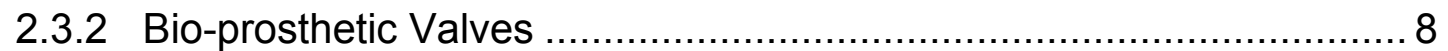

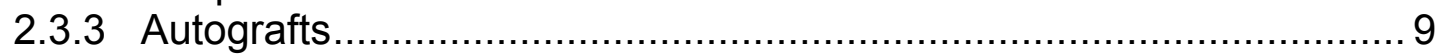

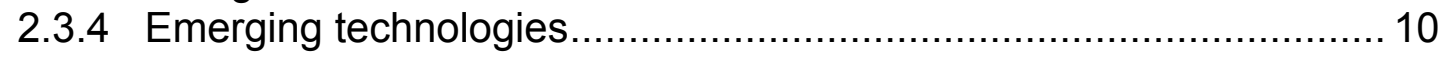

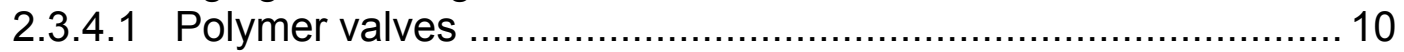

2.3.4.2 Tissue Engineered Heart Valves ......................................... 11

CHAPTER 3: COMPUTATIONAL FLUID DYNAMICS AND ITS

APPLICATIONS IN HEART VALVE STUDIES .......................................... 13

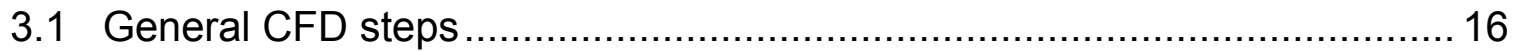

3.2 Moving Boundary Problems.......................................................... 17

3.3 Computer Simulations of mass transport............................................ 22

3.3.1 Overview of governing equations in CFD ................................... 25

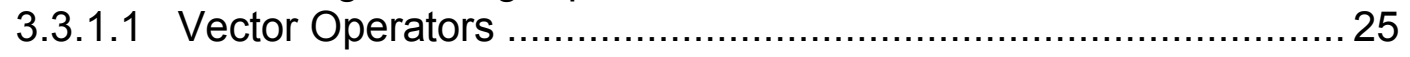

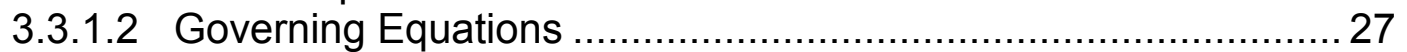

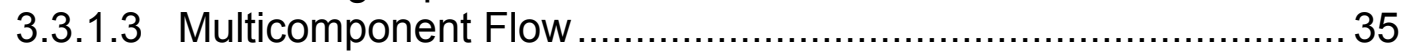

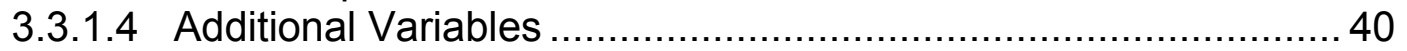

3.3.1.5 Directional Loss Model........................................................ 42

3.3.1.6 General Momentum Sources .............................................. 43

3.3.1.7 Immersed Solids ................................................................. 43

3.4 CFD usage for emerging technologies in Heart Valve Research.............. 44

3.5 Recent Advances in CFD Applications for Heart Valve Research ............. 46

3.5.1 Imaging to Meshing to CFD ..................................................... 46

3.6 Conclusions and Future Outlook ................................................ 58

CHAPTER 4: COMPUTER SIMULATIONS OF

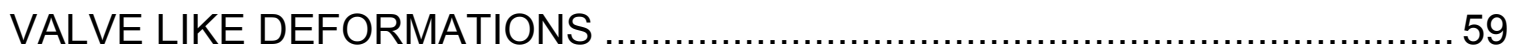

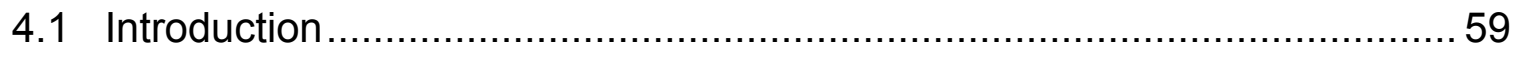

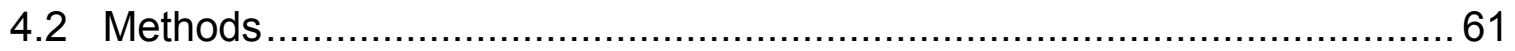

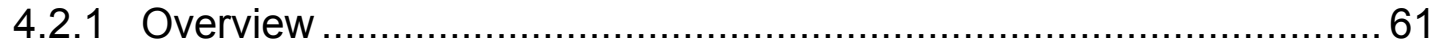

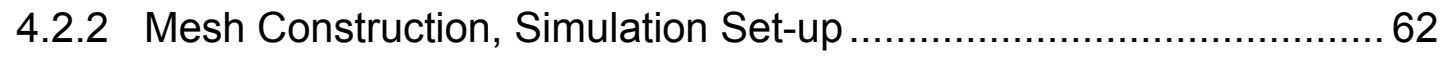

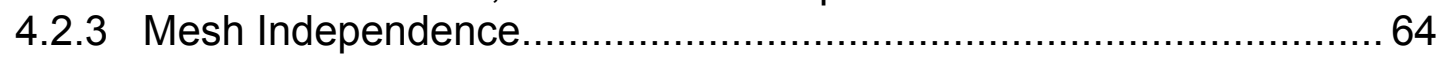

4.2.4 Simulations involving non-moving samples

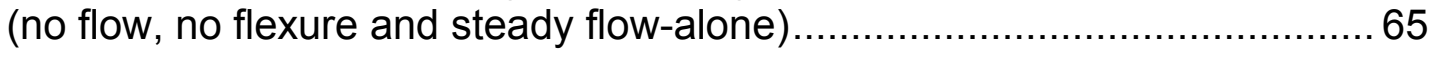


4.2.5 Simulations involving moving samples (cyclic flexure-alone

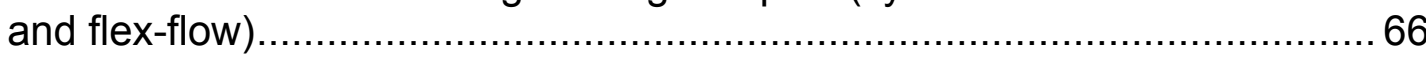

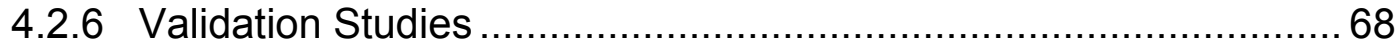

4.2.6.1 CFD Simulation of a moving indentation in a 2-D Channel ........... 68

4.2.6.2 CFD validation of specimen dynamic simulations ........................ 72

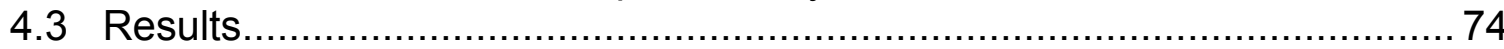

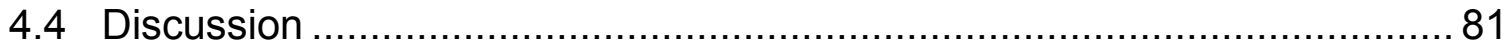

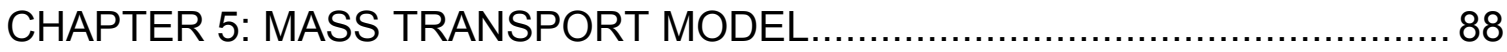

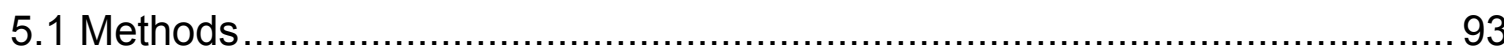

5.1.1 Geometry, Mesh, and Simulation Set-up …….............................. 93

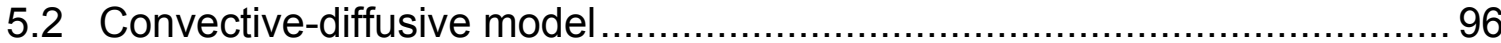

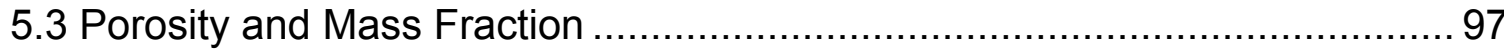

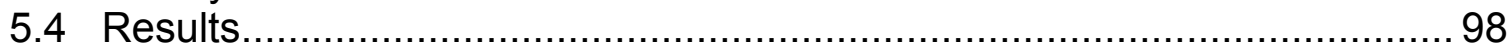

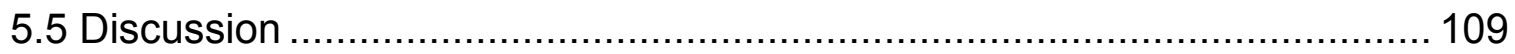

CHAPTER 6: PRELIMINARY EXPERIMENTAL OBSERVATIONS

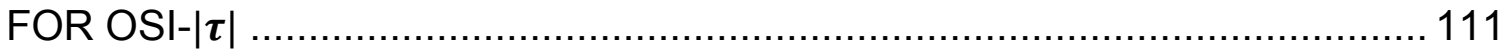

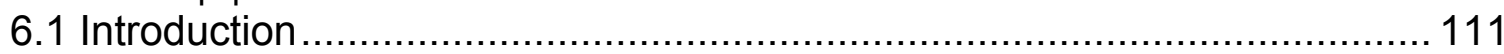

6.2 Methodology for Cell Culture experiments......................................... 112

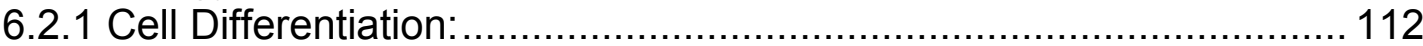

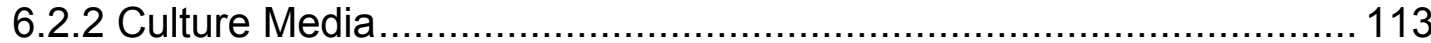

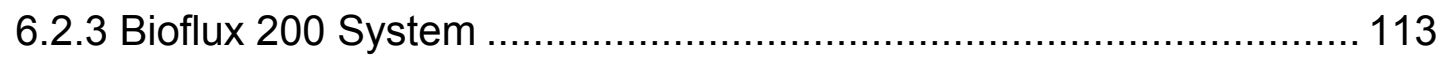

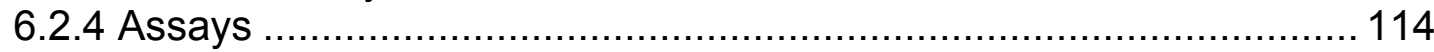

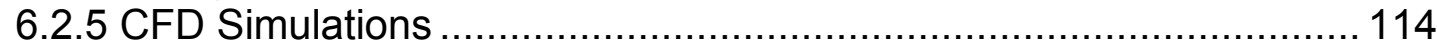

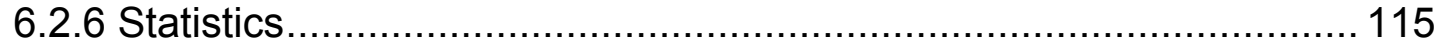

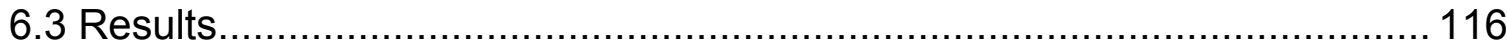

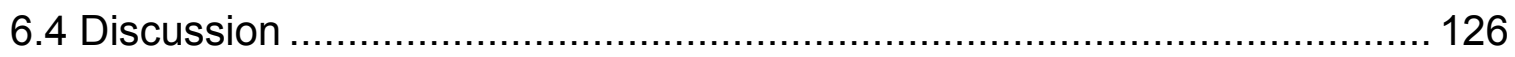

CHAPTER 7: CONCLUSIONS, LIMITATIONS, FUTURE WORK ................... 129 


\section{LIST OF TABLES}

TABLE

PAGE

Table 1: Summary of the advantages and disadvantages of different CFD methodologies for moving boundary problems.

Table 2. Boundary conditions used for the CFD transport model .....................95

Table 3. Parameters used in the mass transport model. ..................................97

Table 4. At 75\% porosity: Anova results for normalized glucose.................... 106

Table 5. At 75\% porosity: Anova results for normalized glucose.................... 106

Table 6. At 5\% porosity: Anova results for normalized glucose ..................... 107

Table 7. At 5\% porosity: Multiple comparisons using Tukey's test for normalized glucose.

Table 8. At 5\% porosity: Anova results for normalized oxygen...................... 108

Table 9. At 5\% porosity: Multiple comparisons using Tukey's test for normalized oxygen.

Table 10. Anova results for cell count from control, steady and pulsatile flow conditions

Table 11. Post hoc tests. Multiple comparisons using Tukey's test for cell counts from control, steady and pulsatile flow conditions. 


\section{LIST OF FIGURES}

FIGURE

PAGE

Figure 1. Mechanical and Biological Heart valves...................................... 7

Figure 2. Tissue Engineering Approach .................................................. 12

Figure 3. Custom made mechanical conditioning bioreactor for

HVTE studies (Engelmayr et al., 2006). Modes: Flow, stretch, flexure.

Figure 4. Computational fluid dynamic simulations on native heart valve (a) and rectangular (b) Geometries (Ramaswamy et al., 2010).

Figure 5. The formation of coherent structures inside the left ventricular chamber during diastole in (a). The small scale structures are advected into the aorta during systole in (d). The red dot in the inset shows the time instance in the cardiac cycle. (From Le TB, Sotiropoulos F. Fluid-structure interaction of an aortic heart valve prosthesis driven by an animated anatomic left ventricle. J Comput Phys. 2013(244):41-62. With permission from Elsevier) ..... 49

Figure 6. The patterns associated with accumulative shear stress applied on particles passing through: (a) a normal BMHV and (b) a dysfunctional BMHV.(From Shahriari S, Maleki H, Hassan I, Kadem L. Evaluation of shear stress accumulation on blood components in normal and dysfunctional bileaflet mechanical heart valves using smoothed particle hydrodynamics. J Biomech. 2012;45(15):2637-2644. With permission from Elsevier).....

Figure 7. FSI simulations with peak $\operatorname{Re}=6000$ : Comparison of instantaneous local maximum shear sloc contours over the midplane of the valve for an MHV vs. BHV implanted in a straight aorta at time $t=(a) 52$, (b) 93 , (c) 186, and (d) $334 \mathrm{~ms}$ within the cardiac cycle. (From Borazjani I. Fluid-structure interaction, immersed boundary-finite element method simulations of bioprosthetic heart valves. Comput Methods Appl Mech Eng. 2013;257(0): 103-116. With permission from Elsevier).

Figure 8. FSI simulations with peak $\mathrm{Re}=6000: 3 \mathrm{D}$ wake structure visualized by the iso-surfaces of q-criteria for a MHV vs. BHV implanted in a straight aorta at time $\mathrm{t}=$ (a) 52, (b) 93 , (c) 186, and (d) $334 \mathrm{~ms}$ within the cardiac cycle. (From Borazjani I. Fluid-structure interaction, immersed boundary-finite element method simulations of bio-prosthetic heart valves. Comput Methods Appl Mech Eng. 2013;257(0):103-116. With permission from Elsevier) .

Figure 9. FSI simulations of BHV: The shape of the BHV and the magnitude of the non-dimensional second Piola-Kirchhoff stress S on the BHV at time 
instants $t=$ (a) 52, (b) 93 , (c) 186, and (d) 334 ms within the cardiac cycle. (From Borazjani I. Fluid-structure interaction, immersed boundary-finite element method simulations of bio-prosthetic heart valves.

Comput Methods Appl Mech Eng. 2013;257(0):103-116. With permission from Elsevier)

Figure 10. (A) Peristaltic pump used to induce flow through the FSF bioreactor. (B) FSF bioreactor set up. (C) Single FSF bioreactor chamber. (D) Bioreactor fluid domain showing the position of the three rectangular specimens.

Figure 11: (a) U-shaped bioreactor showing the three samples.

(b) Front view of the bioreactor inlet, outlet and sample position. The Table indicates the parameters for steady and pulsatile simulations.

Figure 12: OSI distribution along the centerline of samples for (a): Inner wall and (b) Outer wall.Notice how the OSI variability was lowest along the inner and outer walls of straight samples $(\mathrm{N}=3)$ subjected to pulsatile flow. The average OSI in the inner surface was found to be 0.2627 , $0.2121,0.2396$, and 0.1009 for straight, semi-bent, fully-bent, and quasi-static, respectively. In that same order, the outer wall values were $0.2689,0.1859,0.2159$ and 0.2485 (Salinas et al., 2014a; Salinas et al., 2014b). 57

Figure 13: a) Shows a single bioreactor chamber connected to media source. The media is pumped into the bioreactor by a peristaltic pump (Boronyak et al., 2009; Ramaswamy et al., 2009a; Ramaswamy et al., 2009b; Ramaswamy et al., 2010; Ramaswamy et al., 2011; Ramaswamy et al., 2014). An external actuator attached to one end of the samples provides movement. b) Shows an inside look of the bioreactor. The chamber can hold three specimens. Each sample has one end fixed while the other is allowed to move linearly by means of the actuator.

Figure 14: a) Depicts the entire fluid domain of the bioreactor chamber. Note the three scaffolds in a straight configuration and the plane (in red) at $x=0.075 \mathrm{~m}$ corresponding to the longitudinal center line of the samples. b) Cross section $(x=0.0175 \mathrm{~m})$ of the structured mesh (which consisted of 441,370 elements and 472,728 nodes) showing the distribution of quadrilateral elements and nodes. Note that the element density is higher around the samples and lower as it approaches the outlet. c) A close-up of the mesh around a single sample. The elements around the vicinity of the samples were aligned to conform to the flexing shape of the specimens. d) Close-up of the sample edge during $t=0 \mathrm{~s}, t=0.25 \mathrm{~s}, \mathrm{t}=0.5 \mathrm{~s}, \mathrm{t}=0.75 \mathrm{~s}, \mathrm{t}=1 \mathrm{~s}$. 
Figure 15. Schematic representation of the 2-D channel used in the experiment

Figure 16. CFD Mesh - 20,000 Hexagonal Elements. 0 seconds (undeformed).

Figure 17. CFD Mesh - 20,000 Hexagonal Elements. 70

Figure 18. Simplified sketch of the flow patterns observed by (Pedley and Stephanoff, 1985). Source: Pedley T.J. and Stephanoff K.D., (1985), Flow along a Channel with a Time-Dependent Indentation in One Wall: the Generation of Vorticitv Waves. Journalof Fluid Mechanics, Volume 160, pp.337-367.

Figure 19. CFD replication of flow experiments performed by (Pedley and Stephanoff, 1985).

Figure 20: Fluid-induced, time-averaged axial shear stresses over one cycle on specimen inner and outer walls for the following six cases: i) no flow and no flexure ii) steady flow-alone (straight sample), iii) steady flow-alone (semi-bent sample), iv) steady flow-alone (fully-bent sample), v) cyclic flexure-alone and vi) flex-flow states. Shear stresses were found to be higher along the outer wall except in the case of no flow and no flexure. The highest shear stresses were observed around the vertex of the sample. In comparing the two dynamic cases (v) versus (vi), the flex-flow state displayed much higher shear stress values.

Figure 21: Contour of velocity magnitudes and velocity streamlines in the plane $(x=17.5 \mathrm{~mm})$ surrounding the specimens for the following cases: a) steady flow-alone (straight sample), b) cyclic flexure-alone and c) flex-flow states. The inlet velocity for a) and c) was set to $0.022 \mathrm{~m} / \mathrm{s}$ while for b) it was set to $0 \mathrm{~m} / \mathrm{s}$. A large degree of vortices formed in cyclic flexure-alone case, but the magnitude of velocity field was clearly lower when compared to steady flow-alone and flex-flow cases. It was also noted that the flow physics during the specimen flexing and straightening events (in both b) and c) at the instance when the specimen shape was identical, i.e., at $t=0.25$ and $0.75 \mathrm{~s}$ respectively were not the same.

Figure 22: a) OSI on the inner and outer specimen surface for the dynamic cyclic flexure-alone and flex-flow cases. The node averaged OSI along the inner walls were 0.433 (cyclic flexure-alone) and 0.230 (flex-flow) while for the outer walls the OSI was 0.313 (cyclic flexure-alone) and 0.076 (flex-flow). b) OSI-scaled shear stress magnitude (osi-| $\boldsymbol{\tau} \mid$ ) on the inner and outer specimen surface for the dynamic cyclic flexure-alone and flex-flow cases. The node averaged osi- $|\tau|$ along the inner walls were $0.01 \mathrm{~Pa}$ (cyclic flexure-alone) and 
$0.066 \mathrm{~Pa}$ (flex-flow) while for the outer walls the osi-| $|\boldsymbol{t}|$ was $0.016 \mathrm{~Pa}$ (cyclic flexure-alone) and $0.028 \mathrm{~Pa}$ (flex-flow). Effectively the osi- $|\tau|$ metric served to combine both the time averaged and oscillatory shear stress components, demonstrating that its magnitude was much lower in cyclic flexure-alone states compared to flex-flow states, even though the OSI of the former was substantially higher compared to the latter.

Figure 23: For the following three cases of 1) steady flow-alone (straight sample), 2) cyclic flexure-alone and 3) flex-flow states, the specimen area

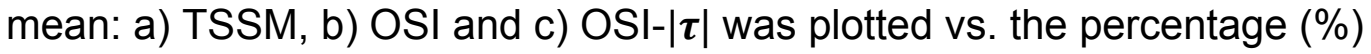
collagen increase. In a) b) and c), the \% increase in collagen was observed relative to the average of the sum of no flexure and no flow, steady flow-alone (straight sample) and cyclic flexure-alone, as previously reported by (Engelmayr et al., 2006) Only the OSI-| $\boldsymbol{\tau} \mid$ parameter demonstrated a convincing correlation to the collagen production found in flex-flow states (Engelmayr et al., 2006), which resulted in significantly higher engineered tissue collagen content.

Figure 24: a) Depiction of the entire fluid domain of the bioreactor chamber. Note the three scaffolds in a straight configuration and the plane (in red) at $x=0.0175 \mathrm{~m}$ corresponding to the longitudinal center line of the samples. b) Cross section $(x=0.0175 \mathrm{~m})$ of the mesh. Note that the element density is higher inside the samples in order to better capture mass transport events.

c) A close-up of the mesh around a single sample.

Figure 25. Contour of velocity magnitudes and velocity streamlines in the plane $(x=17.5 \mathrm{~mm})$ surrounding the specimens during flex-flow states

Figure 26. Contour of velocity magnitudes and velocity streamlines in the plane $(x=17.5 \mathrm{~mm})$ surrounding the specimens during cyclic flexure-alone states.

Figure 27. Contour of velocity magnitudes and velocity streamlines in the plane $(x=17.5 \mathrm{~mm})$ surrounding the specimens during steady flow-alone states.

Figure 28. Time averaged axial velocities (plane $x=-0.0175 \mathrm{~m}$ ) from line at $0.00015 \mathrm{~m}$ below the inner wall.

Figure 29. Time averaged axial velocities (plane $x=-0.0175 \mathrm{~m}$ ) from line at $0.00015 \mathrm{~m}$ above the outer wall. 100

Figure 30. Time averaged normalized mass fraction for glucose at different porosity percentages. 
Figure 31. Time averaged normalized mass fraction for oxygen at different porosity percentages.

Figure 32 . At $75 \%$ porosity: Area and time averaged normalized mass fraction at sample longitudinal wall at $x=-0.0175 \mathrm{~m}$ for glucose .

Figure 33 . At $75 \%$ porosity: Area and time averaged normalized mass fraction at sample longitudinal wall at $x=-0.0175 m$ for oxygen. 104

Figure 34 . At $5 \%$ porosity: Time averaged normalized glucose mf (sample longitudinal wall at $x=-0.0175 \mathrm{~m}$ ) for cases of flex-flow, cyclic flexure-alone, steady flow-alone and control.

Figure 35. Time averaged normalized oxygen $\mathrm{mf}$ (sample longitudinal wall at $x=-0.0175 \mathrm{~m}$ ) for cases of flex-flow, cyclic flexure-alone, flow-alone and control.

Figure 36 . At $5 \%$ porosity: Area and time averaged normalized mass fraction at sample longitudinal wall at $x=-0.0175 \mathrm{~m}$ for glucose .

Figure 37 . At $5 \%$ porosity: Area and time averaged normalized mass fraction at sample longitudinal wall at $x=-0.0175 \mathrm{~m}$ for oxygen. 106

Figure 38. Velocity waveform with a velocity average of $0.199386 \mathrm{~cm} / \mathrm{s}$. It was used as the inlet velocity for the transient pulsatile simulations at 2 millisecond intervals.

Figure 39. Control group. Cell attached to the walls of the bioflux 200 system day 1 .

Figure 40. Control group. Cell attached to the walls of the bioflux 200 system day 5 .

Figure 41. Control group. Cell attached to the walls of the bioflux 200 system day 6 .

Figure 42. Control group. Cell attached to the walls of the bioflux 200 system day 7 .

Figure 43. Steady flow group. Cell attached to the walls of the bioflux 200 system day 1 .

Figure 44. Steady flow group. Cell attached to the walls of the bioflux 200 system day 2. 
Figure 45. Steady flow group. Cell attached to the walls of the bioflux 200 system day 3 .

Figure 46. Steady flow group. Cell attached to the walls of the bioflux 200 system day 4 .

Figure 47. Steady flow group. Cell attached to the walls of the bioflux 200 system day 5 .

Figure 48 Steady flow group. Cell attached to the walls of the bioflux 200 system day 6 .

Figure 49. Steady flow group. Cell attached to the walls of the bioflux 200 system day 7 .

Figure 50. Pulsatile flow group. Cell attached to the walls of the bioflux 200 system day 1 .

Figure 51. Pulsatile flow group. Cell attached to the walls of the bioflux 200 system day 2 .

Figure 52. Pulsatile flow group. Cell attached to the walls of the bioflux 200 system day 3 .

Figure 53. Pulsatile flow group. Cell attached to the walls of the bioflux 200 system day 4 .

Figure 54. Pulsatile flow group. Cell attached to the walls of the bioflux 200 system day 5 .

Figure 55. Pulsatile flow group. Cell attached to the walls of the bioflux 200 system day 6 .

Figure 56. Pulsatile flow group. Cell attached to the walls of the bioflux 200 system day 7 .

Figure 57. Bar chart showing the number of cells during control, steady, and pusatile flow experiments.

Figure 58. Genes expressed after control conditions. 124

Figure 59. Genes expressed after steady flow conditions. 125

Figure 60. Genes expressed after pulsatile flow conditions 125 
Figure 61. Contours of a) OSI (Rath et al., 2014) , b) Time averaged shear stress, and c) OSI- $|\tau|$ of the bioflux 200 rectangular channel seeded with BMSCs.

Figure 62. Idealized velocity waveform................................................... 132

Figure 63. Contours of the inner and outer walls of the fixed rectangular

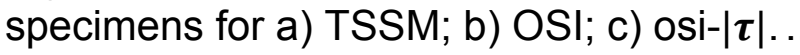




\section{CHAPTER 1: INTRODUCTION}

Heart valve disease is a major health care concern around the world. It is estimated that over 290,000 damaged heart valves are replaced with prosthetic valves annually, and it is expected to triple in the next four decades (Gandaglia et al., 2011). Native heart valves (NHVs) are multilayered highly adaptable and highly regulated structures. NHVs open and close nearly three billion times over the time course of the average human lifespan, and its motion is driven by surrounding hemodynamic forces (Schoen, 2008). NHVs are subjected to complex time-varying stresses due to combinations of flow, stretch and flexure (FSF), and their closing is aided by flow vortices behind the cusps on the arterial side (Schoen, 2008). NHVs are tightly regulated with continuous tissue remodeling activity capable of adapting to different hemodynamic loads (Kleinstreuer, 2006).

The use of prosthetic heart valves substantially restores valve functionality. However, mechanical heart valves (MHVs) may result in hemolysis and possible platelet activation due to abnormal hemodynamics (Ratner et al., ). Similarly, biological heart valves (BHVs) can fail due to calcification and immune rejection (Kleinstreuer, 2006; Silverthorn, 2005). Other emerging techniques such as polymeric substitutes need to be further evaluated. Heart valve tissue engineering aims at the development of an in vitro implant with similar mechanical properties and anatomy as native valves. Moreover, a primary goal of this approach is to promote engineered tissue remodeling and growth (Ramaswamy et al., 2008). However, no long term/ perpetual reliable engineered 
valve has been obtained thus far due to the complexity in mimicking the environments that native valves are exposed to (El-Hamamsy et al., 2010; Gandaglia et al., 2011; Sutherland et al., 2005). The use of computational fluid dynamics (CFD) has provided with insights on these complex time varying flow environments that NHVs are exposed to, but complete and exhaustive data from dynamic simulations are still needed today as well as simulation data correlation to experimental findings. The objectives of this proposal are to study the effects of valve-like movement on resulting fluid-induced stresses and mass transport efficiency (Oxygen and glucose) in developing engineered heart valve tissues. Our underlying hypothesis is that specimen movement effects will result in heighted, fluid-induced, oscillatory shear stresses on the specimens as well as an enhancement in the mass transport of key gas and nutrient species to the developing heart valve tissues. We will test this hypothesis by carrying out the following specific aims:

\section{Specific Aims}

\section{Aim 1: Identify fluid-induced shear stress, velocity, and oscillatory stress distributions on engineered tissue specimens during valve-like deformations.}

Valve-like movement introduces spatial and time varying effects that may be important for the development of tissue engineered heart valve (TEHV) constructs. We will perform specimen dynamic and static steady state CFD simulations, and we will evaluate the flow physics surrounding the constructs. 


\section{Aim 2: Quantify oxygen and glucose concentration profiles on}

engineered tissue specimen geometry during valve-like deformations. Valve-like movement produces varying velocities and pressures around and within the sample. This may aid in the flux of nutrients (glucose and oxygen) through the walls and into the cells and tissue. With data from the dynamic simulations, we will determine if specimen movement improves mass transport of 1 gas (oxygen) and 1 nutrient species (glucose) through the constructs.

\section{Aim 3: Co-relate findings derived from aims 1 and 2 to supporting experiments on engineered tissue formation under static and dynamic tissue culture states, specifically engineered collagen production by the scaffold-seeded, bone marrow derived, mesenchymal stem cells.} Spatially and time varying fluid-induced stresses and mass transport (Oxygen and glucose) effects may improve tissue formation in TEHV constructs but the underlying mechanisms yielding this improvement are not known. To provide further insights on this process, we will compare transport phenomena-related data from the simulations (aims 1 and 2) with collagen assay experimental data obtained from the literature. 


\section{CHAPTER 2: HEART VALVES ANATOMY, DISEASE AND PROSTHETIC DEVICES}

\section{$2.1 \quad$ Heart Valves}

Healthy heart valves are essential for proper blood circulation. Their role is to ensure uni-directional blood flow in the circulation. They consist of different tissue layers and are capable of adapting and remodeling their tissue composition according to the hemodynamic environments surrounding them (Waterbolk et al., 2006; Westhoff-Bleck et al., 2011; Zilla et al., 2008). The mitral and tricuspid valves are located between the atria and ventricles while the pulmonary valve is found intersecting the right ventricle and the pulmonary artery. The aortic valve resides between the left ventricle and the aorta.

When blood enters the heart through the vena cava into the right atrium, it creates an increase in intra-chamber pressure. When the right atrial pressure exceeds the pressure in the right ventricle, the tricuspid valve opens thereby allowing blood to flow into the right ventricle. Simultaneously, the mitral valve also opens and permits blood to flow from the left atrium into the left ventricle. When the right ventricular pressure exceeds the pressure of the pulmonary arteries, the pulmonary valve opens, allowing blood to flow towards the lungs permitting exchange of oxygen and carbon dioxide. Concurrently, the aortic valve allows oxygen-rich blood to flow to the aorta and towards systemic organs and tissues. It closes once the pressure in the aorta exceeds the ventricular pressure.

Over the course of the average human lifespan, native heart valves (NHVs) open and close an estimated three billion times (Schoen, 2008). During 
each heartbeat, they are subjected to different hemodynamic loads in the form of flow, stretch and flexure (Ramaswamy et al., 2010; Salinas et al., 2014a; Salinas et al., 2014b). In past decades, NHVs have been thought to be passive structures. However, recent studies have shown tremendous cell and tissue activity occurring on its surface and within its layered structure (Ramaswamy et al., 2010; Sacks et al., 2009a; Sacks et al., 2009b; Salinas et al., 2014a; Salinas et al., 2014b).

\subsection{Heart Valve Disease}

For simplicity, we refer from this point forward to any aberrant condition caused by anomalous structures and/or disease manifestation on heart valves as heart valve disease (HVD). HVD can be caused by immune disorders, trauma to the valve or surrounding tissues, hypertention, degenerative valve calcification, endocarditis, rheumatic fever and by congenital birth defects (Bernard, 2008; Dal Pan et al., 2005; Elkayam and Bitar, 2005; Frederick J., 2006; lyengar et al., 2004; Lorna, 2010; Mohammadi and Mequanint, 2011; Neuenschwander and P. Hoerstrup, 2004; Shahbudin H., 2010; Siu and Silversides, 2010; Talwar et al., 2005; Waterbolk et al., 2006; Westhoff-Bleck et al., 2011). It can be diagnosed by listening to the opening and closing of the valves through a stethoscope for the identification of heart murmurs. Other methods of detection include echocardiography, electrocardiogram, chest X-ray, cardiac catheterization, stress testing, or magnetic resonance imaging (Elkayam and Bitar, 2005; lyengar et al., 2004; Lorna, 2010; Siu and Silversides, 2010; Talwar et al., 2005). 
Common symptoms of HVD include aortic valve regurgitation, wherein one or more of the three leaflets do not close properly, thereby causing blood to flow back through the valve into the left ventricle (Baig and Punjabi, 2008; Bernard, 2008; Dal Pan et al., 2005; Elkayam and Bitar, 2005). When regurgitation fractions are large, the left ventricle will overwork to pump more blood out of the heart, which may result in left ventricular hypertrophy and possibly heart failure, if left untreated. Another condition, aortic valve stenosis is a state in which the valve leaflets become stiff or even fuse together resulting in narrowing of the aortic valve (Shahbudin H., 2010; Talwar et al., 2005; Waterbolk et al., 2006). This in turn causes a reduction in the valve orifice area which exhibits disturbances in the normal hemodynamics (lyengar et al., 2004; Lorna, 2010; Mohammadi and Mequanint, 2011; Siu and Silversides, 2010; Talwar et al., 2005; Waterbolk et al., 2006; Westhoff-Bleck et al., 2011). Valve stenosis can be congenital or be caused later in life due to, for example, onset of calcification of leaflets. Importantly, the workload on the left ventricle increases considerably which can result in serious complications, as mentioned previously.

\subsection{Valve Prosthetics and Substitutes}

\subsubsection{Mechanical Valves}

Mechanical heart valves (MHV) are prosthetic devices made out of high strength, artificial materials such as titanium and pyrolytic carbon (Mohammadi and Mequanint, 2011; Shahbudin H., 2010; Sotiropoulos and Borazjani, 2009). The most common MHV designs include the ball and cage, tilting disk, and the bileaflet (Butcher et al., 2011; Gandaglia et al., 2011; Shahbudin H., 2010; Zilla et 
al., 2008) mechanical heart valve. The bileaflet design (e.g. bileaflet valve from St. Jude Medical) is currently very popular because of its superior hemodynamics, such as the ability to exhibit low transvalvular pressure gradients (Lorna, 2010; Mohammadi and Mequanint, 2011; Shahbudin H., 2010).

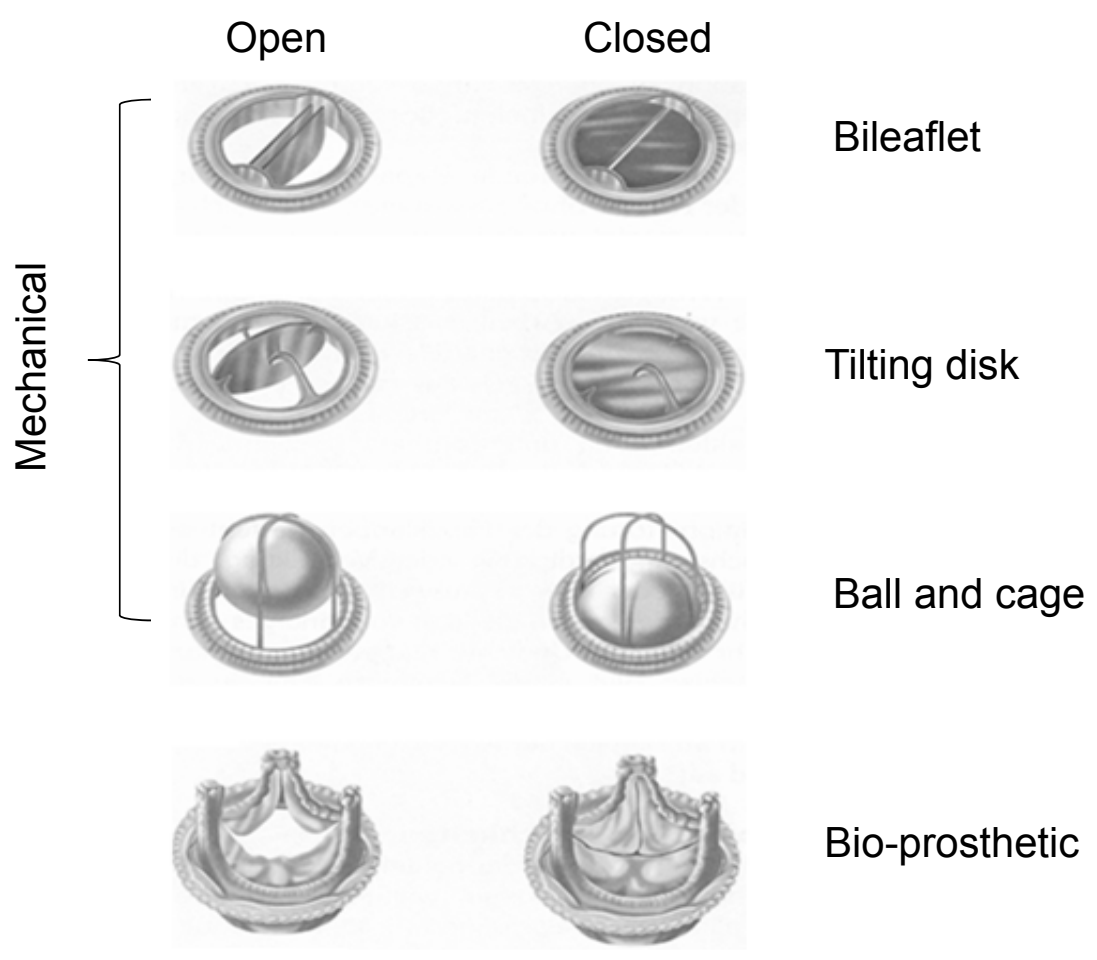

Figure 1. Mechanical and Biological Heart valves.

MHVs have shown to be reliable and long lasting. However, there are some serious limitations that tend to exclude several segments of the population affected by severe HVD. One problem is a predisposition to hemolysis and platelet activation which can trigger thrombus nucleation that could potentially cause blood flow disturbances (Arcidiacono et al., 2005; David and Hsu, 1996a; David and Hsu, 1996b). Furthermore, blood clots could potentially separate from the valve surface and cause a stroke if the clot migrates to the vasculature in the 
brain. Anticoagulation therapy (e.g. heparin) is required for as long as the MHV is implanted. The use of MHVs are mainly suggested for adults and are not recommended for young children who are prone to injury, women who wish to have children, individuals who cannot tolerate anticoagulation medication (e.g. heparin) and other sub-population groups who are at risk for traumatic episodes of bleeding (Baig and Punjabi, 2008; Talwar et al., 2005).

\subsubsection{Bio-prosthetic Valves}

Bio-prosthetic valves (BPVs) are created by forming the native tissue found in animals (Neuenschwander and P. Hoerstrup, 2004; Sha, 2010) into a valve-shaped construct. Sources of animal tissue include the porcine aortic valve and the bovine pericardium (Neuenschwander and P. Hoerstrup, 2004; Zilla et al., 2008). BPVs offer superior hemodynamics over any mechanical valve including the bileaflet design. However, they are pre-disposed to leaflet calcification and early structural breakdown (Ramaswamy et al., 2010; Salinas et al., 2014a; Salinas et al., 2014b).

Homografts, derived from human cadavers serve as allografts (Anastasiadis et al., 2004; Shahbudin H., 2010). The first aortic valve replacement with a homograft occurred in 1962 by Ross (Ungerleider et al., 2010). These substitutes do not pose a risk of coagulation problems thereby eliminating the need for anticoagulation therapies (Anastasiadis et al., 2004). Other advantages include an absence of hemolysis, very good hemodynamics, good implant integration, resistance to infection and a low trans-valvular pressure 
(Anastasiadis et al., 2004; Shahbudin H., 2010). However homografts are also calcification prone and are very limited in supply.

\subsubsection{Autografts}

Valve autografts are valve implants wherein a pulmonary valve is dissected and secured in the aortic position to compensate for a malfunctioning aortic valve. Meanwhile a homograft is implanted in the pulmonary artery to substitute for the native valve that is removed. This process, clinically known as the Ross procedure has demonstrated extensive benefits (Athanasiou et al., 2004; Brown et al., 2004; Gamez et al., 2011; Jaggers et al., 1998; Khwaja et al., 2005; Pretorius et al., 2008; Rus et al., 2004; Sarioglu et al., 2003; Slater et al., 2005; Stelzer, 2011; Ungerleider et al., 2010) such as: i) no necessity for lifelong coagulation therapy, ii) minimal risk of calcification, iii) durability, iv) minimal scaring, v) adequate hemodynamics, vi) growth potential and vii) a low risk of infections and tissue rejection. However, the critical shortcomings (Brown et al., 2004; Gamez et al., 2011; Jaggers et al., 1998; Khwaja et al., 2005; Pretorius et al., 2008; Rus et al., 2004; Sarioglu et al., 2003; Slater et al., 2005; Stelzer, 2011; Ungerleider et al., 2010) that restrict this approach are that it: i) requires a high level of technical skill from the surgeon, ii) data to support long-term outcomes is limited, iii) homograft in pulmonary circulation will likely require replacement over human lifetime, iv) it cannot be used in children with structural abnormalities on their pulmonary valve (due to Marfan syndrome, systemic lupus erythematosus and juvenile rheumatoid arthritis). 


\subsubsection{Emerging technologies}

\subsubsection{Polymer valves}

Development of polymer heart valves were investigated several years ago. However, the main challenge presented at that time was material durability, and this caused an abandonment of these technologies (Ghanbari et al., 2009). Recently, this area of research has been revisited due to more advanced state of the art polymer manufacturing technologies and the development of stronger, biocompatible polymers and polymer composite materials (Di Martino et al., 1997; Ghanbari et al., 2009; Kidane et al., 2009; Ramaswamy et al., 2013). There is also a need for developing valve substitutes that can be integrated with minimal invasive techniques, such as percutaneous valve technologies, which polymer valves are thought to be able to meet (Ramaswamy et al., 2012; Shahbudin H., 2010; Vyavahare et al., 1997).

Polymeric implants can be produced in large quantities and in multiple sizes with low manufacturing cost. The polymers of preference are highly biocompatible elastomers that can be formed to resemble valve-like structures and are able to mimic the deformation of native valve leaflets (Ghanbari et al., 2009; Kidane et al., 2009). However much needs to be done in development to ensure that these types of valves are durable long-term and this remains a subject of on-going research. To date, no polymer valve implant has been approved by the FDA. A critical limitation of polymer valves even if thrombosis, calcification, lack of functionality and weak structural properties can be overcome is that they cannot account for somatic growth in children who have severe HVD; 
in other words, the replacement valve cannot grow and remodel with the overall growth of the child. This means that multiple surgical procedures will still be needed for the pediatric patient with severe congenital HVD.

\subsubsection{Tissue Engineered Heart Valves}

The purpose of heart valve tissue engineering is to obtain an in vitro graft with mechanical properties and anatomical-like structures similar to those of native valves as well as to accommodate somatic growth (Martinez et al., 2013; Ramaswamy et al., 2010; Salinas et al., 2014a; Salinas et al., 2014b). This can be potentially achieved by seeding biomaterial scaffolds with autologous cells such as bone marrow derived stem cells (BMSCs) (Kadner et al., 2002; Leor et al., 2005; Perry et al., 2003; Ramaswamy et al., 2010; Salinas et al., 2014a; Salinas et al., 2014b) and subjecting them to mechanical conditioning paradigms until sufficient heart valve cells and tissue growth in vitro has been achieved (Ramaswamy et al., 2014). Suitable cell sources for tissue engineered heart valves (TEHVs) are primary endothelial (ECs) and smooth muscle cells (SMCs) from a vein that can be harvested without compromise to the vasculature (e.g. saphenous vein in the leg) (Schmidt et al., 2010; Siepe et al., 2008) as well as

progenitor cells sources such as periodontal ligament cells (PDLs) derived from wisdom teeth (Huang et al., 2009). Stem cells such as bone marrow derived stem cells (BMSCs) offer the advantage of being obtained in a much less invasive approach and are accessible whenever needed (Engelmayr et al., 2006; Ramaswamy et al., 2010). 

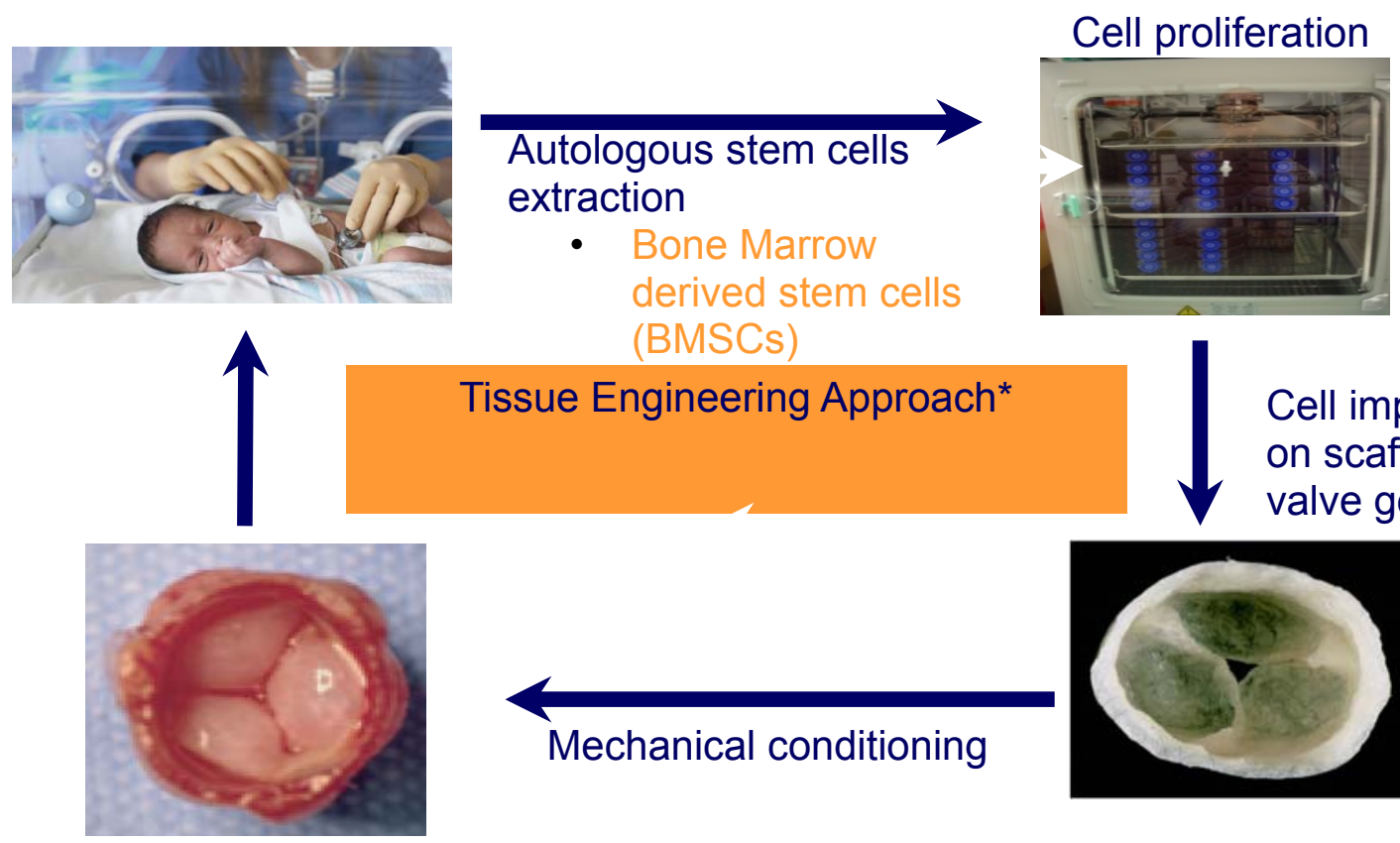

Tissue Engineering Approach*

Cell implantation on scaffold with valve geometry

Engineered heart valve

Figure 2. Tissue Engineering Approach

In terms of the scaffolding components, common biodegradable polymers such as polylactic acid (PLA), polyglycolic acid (PGA) and blends or co-polymers of these two materials, e.g., a 50:50 blend (by weight) of PGA:PLLA nonwoven fabric or poly( lactic-co-glycolic acid), PLGA, have been utilized for heart valve tissue engineering purposes because of their biocompatibility and biodegradable properties (Chen and Wu, 2005; Fong et al., 2006; Leor et al., 2005). However, as cell sources are being identified and as mechanical conditioning protocols are being optimized, it is also critical to at the same time tailor scaffold properties that will demonstrate resilience to cyclic tensile, bending and fluid-induced stress states, while still permitting adequate levels of opening and closing dynamics (Chen and Wu, 2005; Fong et al., 2006; Leor et al., 2005). In this regard, scaffolds made of decellularized animal valves have been utilized (Lichtenberg et 
al., 2006), but unfavorable interactions between the colonizing cells and tissue proteins such as collagen have persisted (Gandaglia et al., 2011; Kleinstreuer, 2006). Recent work has also focused on the idea of using elastomeric substrates that can recapitulate native valve deformation (Ramaswamy et al., 2010; Salinas et al., 2014a; Salinas et al., 2014b). Many challenges need to yet be overcome in optimizing in vitro grown TEHVs that are capable of withstanding in vivo hemodynamic environments and concomitantly permitting growth and tissue remodeling. These technical barriers need to be addressed in order to bring TEHVs from its current research state to that of clinical reality (El-Hamamsy et al., 2010; Gandaglia et al., 2011; Sutherland et al., 2005).

\section{CHAPTER 3: COMPUTATIONAL FLUID DYNAMICS AND ITS APPLICATIONS IN HEART VALVE STUDIES}

Heart valves are subjected to a complex hemodynamic environment. The loading states on native valves during healthy, diseased, pre and post-surgical interventional states as well as prosthetic valves and emerging technologies (e.g. tissue engineered heart valves) can be delineated using computational predictive models. In turn, several insights on how these results may translate to long term functionality can be made. In this context, computational fluid dynamic (CFD) and finite element (FE) model development in the heart valve arena remain a vibrant area of research. FE models are generally applied to evaluate solid surface strains due to cyclical stretching and bending. On the other hand, CFD models examine the effects of fluid induced stresses on the leaflet surfaces. In 
this chapter we focus on CFD models. However we also present fluid structure interaction approaches (FSI) which essentially combines both CFD and FE approaches to delineate both the fluid and solid mechanical responses of the leaflets.

CFD processes for heart valve analyses are based on two numerical methods: The finite element method (FEM) and finite volume method (FVM) (Krause, 1985; Peskin, 1972; Peskin and McQueen, 1980). Both of these techniques subdivide the flow domain into a large number of finite entities, elements, in the case of the FEM, and control volumes, in the case of FVM (Krause, 1985; Peskin, 1972; Peskin and McQueen, 1980). Later, an iterative method is used to obtain the solution for each spatial element or volume. The overall goal is to solve the governing equations of the fluid given by the 3dimensional (3-D) Navier-Stokes equations, by simplifying large complex partial differential equations for the flow physics into algebraic equations (Hose et al., 2006; Onate et al., 2004; Storti et al., 2008). Through this approach, CFD can numerically predict a solution for the nonlinear Navier-Stokes equations which allows the uncovering of the blood flow field surrounding a heart valve (Chandran et al., 2007; Kleinstreuer, 2006).

The usage of CFD to study heart valves can be traced back to the early 1970s. The first models accounted only for 2-dimensional (2D) analysis with oversimplified geometries. 3D models were constructed several years later in the late 1980 s and early 1990 s. We summarize some important milestones in CFD of heart valves and heart valve prostheses here: The flow through a tilting disk in 
a permanently half open position was simulated using a FEM approach by Shim and Cham (Shim and Chang, 1994). Ge et al. (Ge et al., 2005; Ge et al., 2003) performed computer simulations for mechanical valves at Reynolds numbers $(\mathrm{Re})$ describing laminar and turbulent flow; specifically at Reynolds numbers of 750 and 6,000 respectively. The leaflets of this simplified valve were fixed at a completely open state. McQueen et al. (McQueen and Peskin, 1985) described how computer modeling can be used to study design criteria in bileaflet valves with flat and curved leaflets. Lei et al. (Lei et al., 1992) developed a 2D flow model around a Björk-Shiley tilting disk valve under steady state conditions. Simultaneously, Lei et al. (Lei et al., 1992) conducted laser Doppler anemometry (LDA) on a experimental Björk-Shiley tilting disk valve setup that used a mixture of oil and kerosene as the fluid. However, helical vortices observed in the experimental data could not be predicted through the computational results.

Kiris et al. (Kiris et al., 1997) focused on flow past a tilting disk in a fixed position of 30 degrees while ranging Re between two and six thousand. This study used a FVM and solved the 3D Reynolds-averaged Navier-Stokes (RANS) equations that also accounted for turbulence. King et al. (King et al., 1996) described the presence of orifice jets during systole velocities in a CarboMedics ${ }^{\circledR}$ valve. The jet regions were observed to merge towards the aortic walls, and there was evidence of jets in the central flow downstream of the valve leaflets. Experimental results from this study by means of flow visualization and LDA reasonably supported these observations. 
King et al. (King et al., 1997) used a commercially available FEM based software called FIDAP (Fluent, Lebanon, $\mathrm{NH}$ ) to simulate a simplified one-quarter model of a fixed valve at a $R e=1500$. Different leaflet opening angles were used, and it was shown that downstream flow became more centralized and maximum velocity and maximum shear rate increased as well.

\subsection{General CFD steps}

Presently, there are a wide variety of commercially available software packages such as $\mathrm{CFX}{ }^{\circledR}$ (Ansys, Canonsburg, PA), CFD-ACE ${ }^{\circledR}$ (ESI Group, France), $\mathrm{COMSOL}^{\circledR}$ (Burlington, MA), FLEX PDE ${ }^{\circledR}$ (Scientific Software Solutions Int, GR - Europe ), FLUENT ${ }^{\circledR}$ (Ansys, Canonsburg, PA), etc. The operating stages of any of these software packages can be described in three basic steps: pre-processing, solving, and post-processing. The pre-processing stage deals with the construction of geometry; meshing parameters such as grid type and cell size; boundary conditions for all inlets, outlets, walls and interfaces, coupling of equations, and incorporation of physical, mechanical and chemical properties (if needed) into the system. The solving stage involves the setting up of the numerical model, selection of the right solver, and monitoring the computed solution. The post processing stage mainly deals with the creation of 2-D and 3-D plots as well as videos in order to present and interpret the results. If general, revisions and updates to the model will need to be done if the results are erroneous due to lack of numerical accuracy. As a result, a large part of the modeling process will require validation of the computational results with a corresponding experiment. 


\subsection{Moving Boundary Problems}

Presently, simulations involving moving interfaces between flowing or deforming media are some of the most challenging problems in computational science (Driessen et al., 2007; Nobili et al., 2008; Vierendeels et al., 2008). In the body, the movement of heart valves represents an active, dynamic system (Gilmanov and Sotiropoulos, 2005; Peskin, 1972). From a modeling perspective the challenges are linked directly to the numerous differential equations that need to be satisfied for different components within the system (e.g. blood, vessel wall, moving leaflets, etc.), and that these solutions need to also be satisfied through mathematical relationships and appropriate boundary conditions that must hold at the interface of two components (Hong et al., 2009; Sotiropoulos and Borazjani, 2009; Vierendeels et al., 2008). As the name implies, moving boundary methods elude to techniques that incorporate the movement of a solid component with the movement of the fluid in evaluating the fluid and in some cases, the solid stresses as well, as a function of the motion of the solid. In particular, $\mathrm{FSI}$ techniques comprise of scenarios where the movement of the solid-liquid interface is unknown in advance and must be determined as part of the solution. However to date, the ability to solve FSI problems accurately is limited largely to idealized cases, is not straight forward and is computationally expensive. As FSI techniques in heart valve research are generally timeconsuming, during the study planning stage, their usage has to be weighed against the added amount of accuracy that will be provided and level of additional insights that can be gained to benefit the clinical problem at hand. 
Initial algorithmic methods included the Eulerian and Lagrangian approach. In the Eularian case, the velocity field is provided for a fixed control volume. The generated grid is fixed and therefore has a grid velocity, $V_{g}=0$. It is suitable for scenarios with small displacement and conversely is not suitable for systems with massive boundary movement such as those that occur in valve leaflets (Kukreja et al., 2008; Oshima et al., 2001; Sacks et al., 2009b). In the Lagrangian method The generated grid can move and therefore has a grid velocity equal to the velocity of the fluid, $V_{g}=V_{f}$. It is suitable when the system can be easily identified (e.g. elastic solid) (Nguyen and Lim, 2007), but it is not appropriate for systems with randomized boundary movement as it cannot treat arbitrary motion which can occur for example in heart valve disease states (e.g. severe mitral valve prolapse) (Oshima et al., 2001; Sacks et al., 2009b).

Two moving boundary algorithms used for modeling of flow in deforming heart valves include the arbitrary Lagrangian-Eulerian (ALE) formulation and the immersed body method (IBM). The ALE formulation can be used to solve problems by incorporating the velocity of the mesh or grid $\mathrm{Vg}$ and coupling it to the velocity of the fluid Vf. Although the ALE approach has been extensively used, it presents the disadvantage that the mesh needs to be continuously deformed and updated as the control volume travels through the complete system. Given this fact, using the ALE approach could result in a large computational expense, especially for 3D FSI problems. The ALE method is most suitable when the definition of a fixed control volume is not sufficient, e.g. 
when dealing with large- displacement moving walls and in some cases, when the grid velocity can be accurately prescribed a priori (e.g. from patient specific medical images) (Sotiropoulos and Borazjani, 2009).

On the other hand, the IBM allows for accurate interpolations at the fluidstructure interface. This method is efficient as it requires lesser to no mesh update. It is flexible and it can be extended to multi-phase flows which makes it suitable for various applications (Kim and Choi, 2006; Zhang and Gay, 2007). Here, the entire fluid computational domain is discretized as an independent mesh. Similarly, the solid computational domain is also discretized as an independent mesh that is immersed inside the fluid domain. Although both domains are independently meshed, their governing equations are coupled by adding body forces to the Navier-Stokes equations that describe the fluid domain (Gilmanov and Sotiropoulos, 2005; Kim and Choi, 2006; Imanov and Sotiropoulos, 2005; Tai et al., 2007; Zhang and Gay, 2007). This creates a noslip boundary effect at the boundary between the fluid and the solid, and the system accounts for these body forces at the start of the simulation until all iterations have been run. In view of the fact that the grid used to discretize the fluid domain is independent and does not have to be deformed or updated, the IBM is mostly appropriate for moving boundary problems where the mesh displacements are not known beforehand, but rather has to be resolved during the simulation.

Hong et al. (Hong et al., 2009) developed a FSI model using the FLUENT commercial CFD package to create a moving-grid with re-meshing 
techniques based on the ALE approach. They wanted to observe the physical interactions of mechanical heart valves to investigate flows interacting with the moving leaflets. Different rotating positions and tilting angles of the bi-leaflet valves were considered. It was shown that the valve rotation had a significant effect on the transvalvular pressure drop and that valve tilting greatly affects the flow direction.

Ge and Sotiropolus (Ge et al., 2003) were the first to successfully use direct numerical simulations of pulsatile flow through bileaftet mechanical heart valves (BHMV). They simulated blood flow through a BHMV embedded within a straight aortic segment using an IBM approach, and they conducted in vitro studies using a similar experimental set-up. Specifically, they discretized the computational fluid domain with a curvilinear mesh, and the immersed bodies inside the mesh were treated as sharp-interface boundaries which were subsequently discretized using an unstructured meshing scheme. The IBMbased model was able to closely match flow data from in vitro laboratory tests. Sotiropolus, et al. (Sotiropoulos and Borazjani, 2009) also used the IBM to perform high-resolution FSI simulations of a physiologic pulsatile flow through a bi-leaflet mechanical heart valve (BMHV) in an anatomically realistic aorta and through an identical BMHV implanted in a straight aorta. The 3D aortic geometries were re-constructed from stacks of magnetic resonance imaging (MRI) 2D images. The comparisons showed that although some of the salient features of the flow remained the same, the specific geometry of the aorta (e.g. from patient to patient) could have a major effect on both the flow patterns and 
the motion of the valve leaflets. It was also shown that higher leaflet shear stresses were observed in the anatomical-like aorta rather than in the straight idealized case.

When the valve material is flexible, there is higher complexity in CFD simulations and therefore it becomes more challenging because the deformation is localized and motions of the leaflets are highly spatially varying. This is the case in the computational modeling of polymer tissue engineered valves. In 1972, Peskin et al. (Peskin, 1972) developed the first successful 2-D model of a simplified mechanical heart valve inside an artery in order to capture the movement of two solid leaflets embedded in a fluid domain. This was achieved by using an IBM approach. Peskin et al. continued to work on improving this method based on a finite difference scheme and later demonstrated its suitability in capturing interactions between deforming bodies in fluids (Peskin, 1972; Peskin and McQueen, 1980). 


\begin{tabular}{|c|c|c|}
\hline Method & Advantages & Disadvantages \\
\hline Eulerian & $\begin{array}{l}\text { Good for fixed system } \\
\text { analysis with low/none } \\
\text { boundary movement } \\
\text { - Computationally } \\
\text { feasible }\end{array}$ & $\begin{array}{ll}\text { - } & \text { Function of space only } \\
\text { - } & \text { Bad for large } \\
\text { deformations } \\
\text { - } & \text { No adaptive mesh } \\
\text { refinement }\end{array}$ \\
\hline Lagrangian & 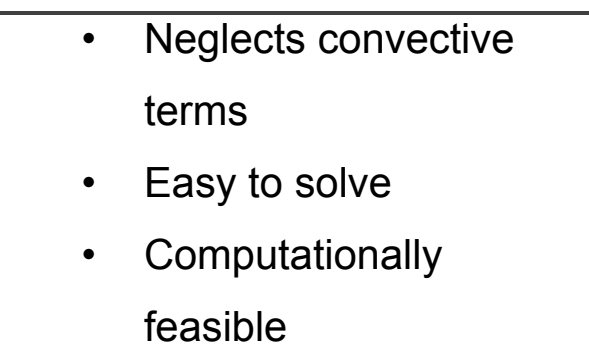 & $\begin{array}{ll}\text { - } & \text { Function of time only } \\
\text { - } & \text { Bad for large } \\
\text { deformations } \\
\text { - No adaptive mesh } \\
\text { refinement }\end{array}$ \\
\hline ALE & $\begin{array}{l}\text { - } \\
\text { Adaptive mesh( } f(x) \text { or } \\
f(t)) \\
\text { - } \text { Good for FSI with low } \\
\text { boundary movement } \\
\text { - } \text { adaptive mesh } \\
\text { refinement if needed }\end{array}$ & $\begin{array}{ll}\cdot & \text { Frequent Mesh } \\
\text { conformation } \\
\text { - } & \text { Takes long time } \\
\text { - } & \text { Expensive for large 3D } \\
\text { systems } \\
\text { - Computationally } \\
\text { challenging }\end{array}$ \\
\hline $\begin{array}{l}\text { Immersed } \\
\text { Body }\end{array}$ & $\begin{array}{ll} & \text { Versatile } \\
\text { - } & \text { Adequate for large } \\
\text { structural displacement } \\
\text { - } & \text { adaptive mesh } \\
& \text { refinement }\end{array}$ & $\begin{array}{l}\text { Requires deep } \\
\text { understanding of CFD } \\
\text { - Computationally } \\
\text { challenging }\end{array}$ \\
\hline
\end{tabular}

Table 1: Summary of the advantages and disadvantages of different CFD methodologies for moving boundary problems.

\subsection{Computer Simulations of mass transport}

In order for proper functioning and survival of cells and tissue, there must be an abundant supply of oxygen and nutrients. To our knowledge, there are no 
studies conducted on the mass transport environments in TEHV constructs. However, extensive mass transport studies have been performed in other cardiovascular tissues such as arteries. Therefore, we have investigated the different mass transport models used in arteries with the aim of finding a robust mass transport model that can be used to study mass transport phenomena happening around TEHV constructs. Arteries and other cardiovascular structures such as heart valves obtain oxygen and glucose by mass transfer between the tissue wall and flowing blood. Mass transfer occurs via by convective mass transport and diffusive processes (Stangeby and Ethier, 2002; Yang and Vafai, 2006). The first is associated with pressure-driven transmural flow while the latter is initiated by species-specific concentration gradients ( $\mathrm{Ai}$ and Vafai, 2006; Ethier and Moore, 1997). These gradients are caused by metabolic uptake and production of proteins by the cells embedded within tissues.

Mass transport through the arteries has been studied using various experimental, analytical, and computational methods. Extensive experimental investigations related to the mass transfer through the arterial wall have been pursued (Chung and Vafai, 2013; de Monte et al., 2013; Denny and Walsh, ; Prosi et al., 2005; Yang and Vafai, 2006; Yang and Vafai, 2008). Prosi et al. (Prosi et al., 2005) have classified these models as three major types, i.e. wallfree, homogenous-wall, and multi-layer-wall models. The simplest models are named wall-free models. This model solves for the blood flow in the lumen while accounting for the effects of the arterial wall simply by means of an appropriate 
set of boundary conditions. As such, the solution is independent of the mass transport processes inside the arterial wall. The values of boundary conditions, for instance the filtration velocity, are usually taken directly from the literature. Because of their simplicity, the wall-free models need a relatively small number of parameters, i.e. the diffusivity in plasma, the overall mass transfer coefficient of the wall with respect to the considered solute and the filtration velocity. Nonetheless, they cannot provide any information on the concentration profiles within the wall. This model has been used for the study of the dynamics of different solutes such as oxygen, albumin, and low-density lipoprotein (LDL) (Lantz and Karlsson, 2012; Yang and Vafai, 2006; Yang and Vafai, 2008).

The Second type of the mass transfer models are the homogeneous- wall models. In these models, the arterial wall is present; however, its complex heterogeneous structure is approximated by a simple homogeneous layer. The properties of wall are usually approximate values based on the assumption that the arterial wall is a homogeneous porous medium. Such models represent a reasonable compromise between the complexity of the input data and the accuracy of the results and are functional in cases where the concentration distribution across the arterial wall is not of primary importance. In addition to the study of hemodynamics and its localizing role, the homogenous-wall model can be used as a tool to investigate the interaction between the hemodynamical parameters and the arterial wall, for instance shear-dependent permeability of the endothelium. Homogeneous-wall models have been used by Ethier and Moore (Ethier and Moore, 1997) to study the concentration of oxygen and LDL 
within the arterial walls. Stangeby and Ethier (Stangeby and Ethier, 2002) modeled the fluid flow within both the lumen and wall of a constricted and axisymmetrically stenosed artery and utilized the resulting flow pattern to study LDL transport from blood to the arterial wall. They coupled luminal blood flow and transmural fluid flow through the solution of Brinkman's model. They prescribed a constant pressure at the adventitial vasa vasorum. In addition, they allowed variations in wall permeability due to the occurrence of plaque.

To date, the most comprehensive model used to characterize the arterial wall is the multi-layer model (Ai and Vafai, 2006; Karner and Perktold, 2000). This model represents the arterial wall to be composed of several heterogeneous porous layers, i.e. endothelium, intima, and media. The multi-layer model is advantageous over the homogeneous-wall model in that it accounts for the particular characteristics and properties of each porous layer. However, it requires a large number of parameters to characterize the transport properties of each layer.

\subsubsection{Overview of governing equations in CFD}

\subsubsection{Vector Operators}

Using a Cartesian coordinate system with $\mathrm{i}, \mathrm{j}$, and $\mathrm{k}$ unit vectors $\nabla$ can be defined as, $\nabla=\left[\frac{\partial}{\partial x}, \frac{\partial}{\partial y}, \frac{\partial}{\partial z}\right] \quad$ Eq. 1

\section{Gradient}

This can be used to define the gradient of a scalar function $\varphi(x, y, z)$ which results in: 
$\nabla \varphi=\frac{\partial \varphi}{\partial x} \mathrm{i}+\frac{\partial \varphi}{\partial y} j+\frac{\partial \varphi}{\partial z} \mathbf{k} \quad$ Eq. 2

The gradient is a generalized derivative that acts over multiple variables of a function.

\section{Divergence}

For a vector function $U(x, y, z)$ where,

$\mathbf{U}=\left[\begin{array}{c}\mathbf{U}_{\mathbf{x}} \\ \mathbf{U}_{\mathbf{y}} \\ \mathbf{U}_{\mathbf{z}}\end{array}\right] \quad$ Eq. 3

The divergence of $U$ can then be defined as:

$\boldsymbol{\nabla} \cdot \mathbf{U}=\frac{\partial U_{\mathrm{x}}}{\partial \mathrm{x}}+\frac{\partial \mathrm{U}_{\mathrm{y}}}{\partial \mathrm{y}}+\frac{\partial \mathrm{U}_{\mathrm{z}}}{\partial \mathrm{z}} \quad$ Eq. 4

The divergence of a vector field describes the flux at a point and therefore allows an inference of source or sink characteristic of the field.

\section{Dyadic Operator}

The dyadic operator (tensor product) of two vectors, $U$ and $V$, is defined as:

$\mathbf{U} \otimes \mathbf{V}=\left[\begin{array}{lll}\mathbf{U}_{\mathbf{x}} \mathbf{V}_{\mathbf{x}} & \mathbf{U}_{\mathbf{x}} \mathbf{V}_{\mathbf{y}} & \mathbf{U}_{\mathbf{x}} \mathbf{V}_{\mathbf{z}} \\ \mathbf{U}_{\mathbf{y}} \mathbf{V}_{\mathbf{x}} & \mathbf{U}_{\mathbf{y}} \mathbf{V}_{\mathbf{y}} & \mathbf{U}_{\mathbf{y}} \mathbf{V}_{\mathbf{z}} \\ \mathbf{U}_{\mathbf{z}} \mathbf{V}_{\mathbf{x}} & \mathbf{U}_{\mathbf{z}} \mathbf{V}_{\mathbf{y}} & \mathbf{U}_{\mathbf{z}} \mathbf{V}_{\mathbf{z}}\end{array}\right] \quad$ Eq. $\mathbf{5}$

Using specific tensor notation the vectors can be combined into a single equation which shows as:

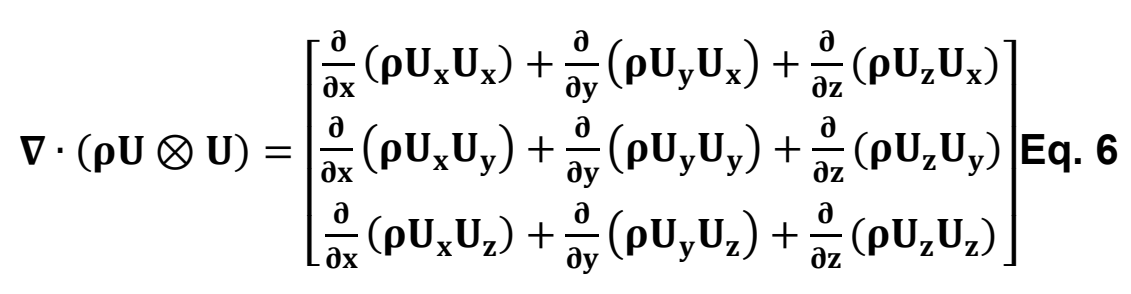


This allows the equations in each dimension to be written into a single equation. The tensor product is an expression of two vectors over a field that is analogous to the product of two scalar values.

\section{Matrix Transposition}

The operator $\mathrm{T}$ is used to denote the transpose of a matrix. So if a matrix defined as:

$\nabla \varphi=\left[\begin{array}{l}\frac{\partial \varphi}{\partial \mathrm{x}} \\ \frac{\partial \varphi}{\partial \mathrm{y}} \\ \frac{\partial \varphi}{\partial \mathrm{z}}\end{array}\right] \quad$ Eq. 7

Then the transposed matrix is:

$[\nabla \varphi]^{T}=\left[\begin{array}{lll}\frac{\partial \varphi}{\partial x} & \frac{\partial \varphi}{\partial y} & \frac{\partial \varphi}{\partial z}\end{array}\right] \quad$ Eq. 8

\section{The Identity Matrix}

The identity matrix (also called the Kronecker Delta function) is defined by:

$\delta=\left[\begin{array}{lll}1 & 0 & 0 \\ 0 & 1 & 0 \\ 0 & 0 & 1\end{array}\right] \quad$ Eq. 9

\subsubsection{Governing Equations}

\section{Transport Equations}

The instantaneous equations of mass, momentum, and energy conservation will be discussed. For turbulent flows an average of the instantaneous equations is used.

\section{The Continuity Equation (Mass)}

The continuity equation is stated as: 
$\frac{\partial \rho}{\partial t}+\nabla \cdot(\rho U)=0 \quad$ Eq. 10

$\rho$ is the fluid density, $\mathrm{t}$ is time, and $\mathrm{U}$ is the fluid velocity vector. The equation states that the amount of mass entering a system is equal to the amount of mass leaving the system.

\section{The Momentum Equations}

The momentum equation is defined as,

$\frac{\partial(\rho \mathbf{U})}{\partial t}+\nabla \cdot(\rho \mathbf{U} \otimes \mathbf{U})=-\nabla p+\nabla \cdot \tau+S_{M}$ Eq. 11

Where the stress tensor $\mathrm{T}$ is related to the strain rate as follows:

$\tau=\mu\left(\nabla \mathbf{U}+(\nabla \mathbf{U})^{\mathrm{T}}-\frac{2}{3} \delta \boldsymbol{\nabla} \cdot \mathbf{U}\right) \quad$ Eq. 12

$U$ represents the fluid velocity vector, $\rho$ is the fluid density, $\mathrm{p}$ is the pressure, $\mathrm{T}$ is the stress tensor and $S_{M}$ is the sum of other forces moving through the system. This equation is referred to as the Navier-Stokes equation and is used to describe the velocity of a fluid at a given space and time. It relates the movement of fluid across a system on the left side of the equation with the pressure, viscous, and other forces acting on that system on the left side of the equation.

\section{The Total Energy Equation}

The total energy equation is defined as:

$\frac{\partial\left(\rho h_{\text {tot })}\right.}{\partial t}-\frac{\partial p}{\partial t}+\nabla \cdot\left(\rho \mathbf{U h}_{\text {tot }}\right)=\nabla \cdot(\lambda \nabla T)+\nabla \cdot(\mathbf{U} \cdot \tau)+\mathbf{U} \cdot S_{\mathrm{M}}+\mathbf{S}_{\mathrm{E}} \quad$ Eq. 13 $h_{\text {tot }}$ is the total enthalpy which can be related to the static enthalpy $h(T, p)$ by, $h_{\text {tot }}=h+\frac{1}{2} U^{2} \quad$ Eq. 14 
This form of the equation is written in terms of enthalpy. However there are many other forms that it can be take. Generally, $\nabla \cdot(\mathrm{U} \cdot \tau)$ the work due to viscous stress is negligible because internal heating by the viscosity of the fluid is negligible. The term $\mathrm{U} \cdot \mathrm{S}_{\mathrm{M}}$ is also neglected this is done to conserve momentum. Thus removing the neglected terms it can be rewritten as:

$\frac{\partial\left(\rho h_{\text {tot }}\right)}{\partial t}-\frac{\partial p}{\partial t}+\nabla \cdot\left(\rho U h_{\text {tot }}\right)=\nabla \cdot(\lambda \nabla T)+S_{E} \quad$ Eq. 15

The left side of the equation represents the work done by the movement of fluid. On the right side the first term represents energy change as a function of temperature $\mathrm{T}$ and the second term is a source that represents other potential sources of energy. The second term potentially could be zero.

\section{The Thermal Energy Equation}

The thermal energy equation is an alternative form of the total energy equation that can be used with low-speed flows. To begin mechanical energy $\mathrm{K}$ is defined as,

$K=\frac{1}{2} U^{2} \quad$ Eq. 16

Taking the dot product of this equation with the momentum equation yields:

$\frac{\partial(\rho K)}{\partial t}+\nabla \cdot(\rho \mathbf{U K})=-\mathbf{U} \cdot \nabla \mathbf{p}+\mathbf{U} \cdot(\boldsymbol{\nabla} \cdot \tau)+\mathbf{U} \cdot \mathbf{S}_{\mathbf{M}} \quad$ Eq. 17

Which when subtracted from the total energy equation yields the thermal energy equation:

$\frac{\partial(\rho h)}{\partial t}-\frac{\partial p}{\partial t}+\nabla \cdot(\rho U h)=\nabla \cdot(\lambda \nabla T)+U \cdot \nabla p+\tau: \nabla U+S_{E}$

Two different assumptions based on the fluid can be used to simplify the equation to the following final form: 
$\frac{\partial(\rho \mathbf{h})}{\partial t}+\nabla \cdot(\rho \mathbf{U h})=\nabla \cdot(\lambda \nabla T)+\tau: \nabla \mathbf{U}+S_{\mathrm{E}}$

If the fluid in question is a liquid in which variable-density effects are negligible then $\mathrm{h}$ can be interpreted as internal energy,

$$
\mathrm{e}=\mathrm{h}-\frac{\mathrm{P}}{\rho}
$$

This allows the previous equation to be rewritten as

$\frac{\partial(\rho e)}{\partial t}+\nabla \cdot(\rho U e)=\nabla \cdot(\lambda \nabla T)-p \nabla \cdot U+\tau: \nabla U+S_{E} \quad$ Eq. 20

Which is equivalent to the final form when e is interpreted as $h$ and $-p \nabla \cdot U$ is neglected

If the fluid is a compressible gas with low Mach flow then $\frac{\partial \rho}{\partial t}$ and $U \cdot \nabla p$ are neglected leading to the same final form. The thermal energy equation is useful for avoiding stability issues in the total energy equation. It cannot be used if proper acoustic behavior is required or in the case of high speed flow.

\section{Equations of State}

The transport equations must be augmented with constitutive equations of state for density and enthalpy in order for the equations to form a closed system. In general the equations take the form:

$\rho=\rho(p, T) \quad$ Eq. 21

$d h=\left.\frac{\partial h}{\partial T}\right|_{p} d T+\left.\frac{\partial h}{\partial p}\right|_{T} d p=c_{p} d T+\left.\frac{\partial h}{\partial p}\right|_{T} d p \quad$ Eq. 22

$c_{p}=c_{p}(p, T) \quad$ Eq. 23 


\section{Incompressible Equation of State}

This is the simplest case. Density is constant and $c_{p}$ at most a function of temperature.

$\rho=\rho_{\text {spec }} \quad$ Eq. 24

$d h=c_{p} d T+\frac{d p}{\rho} \quad$ Eq. 25

$\mathrm{c}_{\mathrm{p}}=\mathrm{c}_{\mathrm{p}}(\mathrm{T})$

\section{Ideal Gas Equation of State}

For an ideal gas, density is calculated from the ideal gas law and $c_{p}$ can be at a function of temperature.

$\rho=\frac{w P_{\text {abs }}}{\mathrm{R}_{0} \mathrm{~T}} \quad$ Eq. 26

$d h=c_{p} d T \quad E q .27$

$c_{p}=c_{p}(T) \quad$ Eq. 28

\section{Real Gas and Liquid Equations of State}

For real gases and liquids a slightly more complex approach is necessary when deriving equations of state. Cubic equations of state are the most convenient means for predicting real fluid behavior. Four versions are used in Ansys: the Standard Redlich Kwong, Aungier Redlich Kwong, Soave Redlich Kwong, and Peng Robinson.

\section{Redlich Kwong Models}

Redlich Kwong variants of the cubic equations of state take the form:

$\mathbf{p}=\frac{\mathbf{R T}}{\mathrm{v}-\mathbf{b}+\mathbf{c}}-\frac{\mathbf{a}(\mathbf{T})}{\mathrm{v}(\mathrm{v}+\mathbf{b})} \quad$ Eq. 29 
$v$ is the specific volume $v=1 / \rho$ and $a, b$, and $c$ are variable that vary from model to model.

\section{The Standard Redlich Kwong Model}

In the Standard Redlich Kwong Model $\mathrm{c}$ is set to zero and a is set to:

$a=a_{0}\left(\frac{T}{T_{c}}\right)^{-n} \quad$ Eq. 30

Where

$\mathrm{n}$ is 0.5

$\begin{array}{ll}\mathrm{a}_{0}=\frac{0.42747 \mathrm{R}^{2} \mathrm{~T}_{\mathrm{c}}{ }^{2}}{\mathrm{p}_{\mathrm{c}}} & \text { Eq. } 31 \\ \mathrm{~b}=\frac{0.08664 \mathrm{RT} T_{\mathrm{c}}}{\mathrm{p}_{\mathrm{c}}} & \text { Eq. } 32\end{array}$

\section{The Aungier Redlich Kwong Model}

The Aungier form differs by having a non-zero value for $\mathrm{c}$ in order to improve the value of isotherms near the critical point. The value of $n$ also differs based on the pure substance. The parameter $\mathrm{c}$ takes the form of:

$\mathbf{c}=\frac{\mathbf{R T}_{\mathrm{c}}}{\mathbf{p}_{\mathbf{c}}+\frac{\mathbf{a}_{0}}{\mathbf{v}_{\mathrm{c}}\left(\mathbf{v}_{\mathrm{c}}+\mathbf{b}\right)}}+\mathbf{b}-\mathbf{v}_{\mathbf{c}} \quad$ Eq. 33

The exponent $\mathrm{n}$ is replaced with a best fit polynomial in terms of the acentric factor $\omega$.

$\mathrm{n}=0.4986+1.1735 \omega+0.4754 \omega^{2} \quad$ Eq. 34

\section{The Soave Redlich Kwong Model}

The Soave Redlich Kwong Model applies to non-polar compounds and improves upon the original by generalizing the attraction term to depend on the acentric factor. This accounts for the fact that molecules are non-spherical and a range of 
vapor pressure data in the development of the temperature dependency of this parameter. $b$ takes the same form as the original and $c$ as well is the same. $a(T)$ however takes the form:

$\mathbf{a}(T)=a_{0}\left(1+n\left(1-\sqrt{\frac{T}{T_{c}}}\right)\right)^{2} \quad$ Eq. 35

Where

$a_{0}$ is just a from the standard model

$n=0.480+1.574 \omega-0.176 \omega^{2}$ Eq. 36

Peng Robinson Model

The Peng Robinson Model takes a similar form as the Redlich Kwong Model but differs slightly and is defined as:

$\mathbf{p}=\frac{\mathbf{R T}}{\mathbf{v}-\mathbf{b}}-\frac{\mathbf{a}(\mathbf{T})}{\mathbf{v}^{2}+2 \mathbf{b v}-\mathbf{b}^{2}} \quad$ Eq. 37

Where

$b=0.0778 \frac{R_{1}}{p_{c}} \quad$ Eq. 38

$\mathbf{a}(T)=a_{0}\left(1+n\left(1-\sqrt{\frac{T}{T_{c}}}\right)\right)^{2} \quad$ Eq. 39

$\mathrm{a}_{0}=0.45724 \frac{\mathrm{R}^{2} \mathrm{~T}_{\mathrm{c}}{ }^{2}}{\mathrm{p}_{\mathrm{c}}} \quad$ Eq. 40

$\mathrm{n}=0.37464+1.54226 \omega-0.26993 \omega^{2} \quad$ Eq. 41

General Equations of State

Equations of state can be user defined in CFX such that they are the following functions
$\boldsymbol{\rho}=\boldsymbol{\rho}(\mathbf{p}, \mathbf{T})$
Eq. 42 
$c_{p}=c_{p}(p, T) \quad$ Eq. 43

Noting that:

$\left.\frac{\partial \mathrm{h}}{\partial \mathrm{p}}\right|_{\mathrm{T}}=\mathrm{v}-\left.\mathrm{T} \frac{\partial \mathrm{v}}{\partial \mathrm{T}}\right|_{\mathrm{p}} \quad$ Eq. 44

In which $v=1 / \rho$. Solving for enthalpy yields:

$d h=c_{p} d T+\left[v-\left.T \frac{\partial v}{\partial T}\right|_{p}\right]_{T} d p \quad E q .45$

Noting that user defined expressions must be thermodynamically consistent

requires that all the mathematical properties for exact differentials be satisfied.

Therefore $d z(x, y)$ is an exact differential defined as:

$\operatorname{dz}(x, y)=M d x+N d y \quad$ Eq. 46

With,

$M=\left.\frac{\partial z}{\partial x}\right|_{y} \quad$ Eq. 47

$N=\left.\frac{\partial z}{\partial y}\right|_{x} \quad$ Eq. 48

Consistency requires that:

$\left.\frac{\partial M}{\partial y}\right|_{x}=\left.\frac{\partial N}{\partial x}\right|_{y} \quad$ Eq. 49

With this in mind, for a general equation of state to be consistent it must obey the following:

$\frac{\partial c_{p}}{\partial p}=\frac{\partial}{\partial T}\left(v-\left.T \frac{\partial v}{\partial T}\right|_{p}\right) \quad$ Eq. 50

Note that it is only valid to specify a general equation of state in which density is a function of T alone. 


\section{Conjugate Heat Transfer}

It is possible to create a solid region in which the equations for heat transfer are solved but those for flow are not. This is known as conjugate heat transfer and the solid region is known as a solid domain. The conservation of energy equation can be used to describe the transfer of heat due to the motion of the solid, conduction, and volumetric heat sources:

$\frac{\partial(\rho h)}{\partial t}+\nabla \cdot\left(\rho U_{s} h\right)=\nabla \cdot(\lambda \nabla T)+S_{E} E q .51$

Where $h$ is the enthalpy, $\rho$ is the density, $\lambda$ is the thermal conductivity of the solid, and $S_{E}$ is an optional volumetric heat source. $U_{S}$ represents the solid velocity which accounts for the motion of the solid with respect to a reference frame.

\subsubsection{Multicomponent Flow}

\section{Scalar Transport Equation}

For a multicomponent fluid the Governing equations are used to solve for the bulk fluid however additional equations must be solved in order to determine how each component of the fluid is transported within the bulk. Each component has an individual conservation of mass equation and after Reynolds-averaging the equation can be expressed in tensor notation as:

$\frac{\partial \widetilde{\rho}_{1}}{\partial t}+\frac{\partial\left({\widetilde{\rho_{1}}}_{\mathrm{U}_{\mathrm{j}}}\right)}{\partial \mathrm{x}_{\mathrm{j}}}=-\frac{\partial}{\partial \mathrm{x}_{\mathrm{j}}}\left(\rho_{\mathrm{i}}\left(\widetilde{\mathrm{U}}_{\mathrm{ij}}-\widetilde{\mathbf{U}}_{\mathrm{j}}\right)-\overline{\rho_{\mathrm{l}} " \mathrm{U}_{\mathrm{j}}^{\prime \prime}}\right)+S_{\mathrm{i}} \quad$ Eq. 52

Where:

$\tilde{\rho}_{\mathrm{i}}$ is the mass average density of the fluid component $\mathrm{i}$ in the mixture

$\widetilde{\mathrm{U}}_{\mathrm{j}}=\sum\left(\widetilde{\rho}_{\mathrm{i}} \widetilde{\mathrm{U}}_{\mathrm{ij}}\right) / \bar{\rho}$ is the mass average velocity field

$\widetilde{U}_{i j}$ is the mass average velocity of fluid component $\mathrm{i}$ 
$\rho_{\mathrm{i}}\left(\widetilde{\mathrm{U}}_{\mathrm{ij}}-\widetilde{\mathrm{U}}_{\mathrm{j}}\right)$ is the relative mass flux

$S_{i}$ is the source term for component $i$ which includes the effects of chemical reactions

If all the terms in the expanded equation are summed over all the components then the result is simply the standard continuity equation:

$\frac{\partial \bar{\rho}}{\partial t}+\frac{\partial\left(\bar{\rho} \widetilde{U}_{j}\right)}{\partial x_{j}}=0$

This is because the reaction rates $S_{i}$ must sum to zero.

The relative mass flux term accounts for differential motion of the individual components. Depending on how it is modeled it can describe the effects of concentration gradients, pressure gradients, external forces, or temperature gradients. The primary effect of motion among the individual components is the concentration gradient and modeling of this effect gives yields diffusion-like term given by:

$\rho_{\mathrm{i}}\left(\widetilde{U}_{\mathrm{ij}}-\widetilde{\mathbf{U}}_{\mathrm{j}}\right)=-\frac{\Gamma_{\mathrm{i}}}{\bar{\rho}} \frac{\partial \widetilde{\rho}_{\mathrm{i}}}{\partial \mathrm{x}_{\mathrm{j}}} \quad$ Eq. 54

The molecular diffusion coefficient, $\Gamma_{\mathrm{i}}$, is assumed to be equal to $\rho \mathrm{D}_{\mathrm{i}}$ where $\mathrm{D}_{\mathrm{i}}$ is the kinematic diffusivity. The mass fraction of component $i$ is then defined as: $\widetilde{\mathbf{Y}}_{\mathrm{i}}=\frac{\widetilde{\rho}_{\mathbf{i}}}{\bar{\rho}} \quad$ Eq. 55

Plugging the previous two equations in to the conservation of mass equation yields:

$\frac{\partial\left(\bar{\rho} \widetilde{Y}_{i}\right)}{\partial t}+\frac{\partial\left(\bar{\rho} \widetilde{U}_{j} \widetilde{Y}_{i}\right)}{\partial x_{j}}=\frac{\partial}{\partial x_{j}}\left(\Gamma_{i} \frac{\partial \widetilde{Y}_{i}}{\partial x_{j}}\right)-\frac{\partial}{\partial x_{j}}\left(\overline{\rho Y_{1}^{\prime \prime} U_{J}^{\prime \prime}}\right)+S_{i} \quad$ Eq. 56

The turbulent scalar fluxes are modeled using the eddy dissipation assumption: 
$-\overline{\rho Y_{1}^{\prime \prime} U_{j}^{\prime \prime}}=\frac{\mu_{t}}{s_{c_{t}}} \frac{\partial \widetilde{Y}_{i}}{\partial x_{j}} \quad$ Eq. 57

$\mathrm{S}_{\mathrm{c}_{\mathrm{t}}}$ is the turbulent Schmidt number. This can then be substituted into the equation and assuming that you have mass weighted averages of $Y_{i}$ the final equation is defined as:

$\frac{\partial\left(\bar{\rho} \widetilde{\mathbf{Y}}_{i}\right)}{\partial t}+\frac{\partial\left(\bar{\rho} \widetilde{\mathbf{U}}_{j} \widetilde{\mathbf{Y}}_{i}\right)}{\partial x_{j}}=\frac{\partial}{\partial x_{j}}\left(\Gamma_{i_{\text {eff }}} \frac{\partial \widetilde{\mathbf{Y}}_{i}}{\partial x_{j}}\right)+S_{i} \quad$ Eq. 58

Where:

$\Gamma_{i_{\text {eff }}}=\Gamma_{i}+\frac{\mu_{t}}{s_{c_{t}}} \quad$ Eq. 59

\section{Constraint Equation for Components}

The CFX-solver solves $\mathrm{N}_{\mathrm{C}}-1$ mass fraction equations for all but one component so the last component is known as the constraint component. The last mass fraction is determined by the equation:

$\sum_{\mathrm{i}=\mathrm{A}, \mathrm{B}, \mathrm{C}, \ldots}^{\mathrm{N}_{\mathrm{c}}}, \mathrm{Y}_{\mathrm{i}}=1 \quad$ Eq. 60

\section{Multicomponent Fluid Properties}

If there is a given volume $V$ of fluid mixture then $M_{i}$ is the mass of component $i$ present in the volume; $\rho_{i}=M_{i} / V$ is then the density of that component; $V_{i}$ is the partial volume of that component that would be taken up by that component of the same mass at the same local temperature and pressure as the mixture. The thermodynamic density of the component is an evaluation of the equation of state under the mixture temperature and pressure is expressed as $\left\langle\rho_{i}\right\rangle=M_{i} / V_{i}$. Since all partial volumes must add up to the total volume, then: 


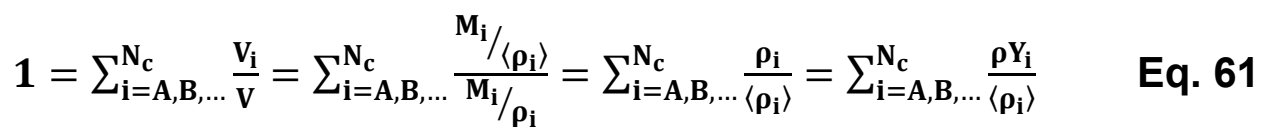

Or:

$\frac{1}{\rho}=\sum_{i=A, B, \ldots}^{N_{c}} \frac{Y_{i}}{\left\langle\rho_{i}\right\rangle} \quad$ Eq. 62

Thus the mixtures density can be calculated with knowledge of the mass

fractions and thermodynamic density of each component.

An arbitrary constitutive property may be calculated from:

$\alpha=\sum_{\mathrm{i}=\mathrm{A}, \mathrm{B}, \ldots}^{\mathrm{N}_{\mathrm{c}}} \mathbf{Y}_{\mathrm{i}} \boldsymbol{\alpha}_{\mathrm{i}} \quad$ Eq. 63

Where $\alpha$ can be the laminar viscosity $\mu$, the specific heat at constant volume $c_{v}$, the specific heat at constant pressure $c_{p}$, and the laminar thermal conductivity $\lambda$.

\section{Energy Equation}

Extending the conservation of energy equation to multicomponent fluids requires adding an additional diffusion term:

$\frac{\partial}{\partial x_{j}}\left[\sum_{i}^{N_{c}} \Gamma_{i} h_{i} \frac{\partial Y_{i}}{\partial x_{j}}\right] \quad$ Eq. 64

For turbulent flows this term is Reynolds-averaged giving:

$\frac{\partial}{\partial x_{j}}\left[\overline{\sum_{1}^{N_{c}}\left(\tilde{\Gamma}_{1}+\Gamma_{1} "\right)\left(\tilde{h}_{1}+h_{1} "\right) \frac{\partial\left(\widetilde{Y}_{1}+Y_{1} "\right)}{\partial x_{j}}}\right]$

Eq. 65

Though this expression contains several fluctuating terms it is not possible with current models to account for these effects individually thus only the mean effect is retained in the calculations. Inserting this extra term into the energy equation yields the following equation:

$\frac{\partial}{\partial t}(\rho H)-\frac{\partial P}{\partial t}+\frac{\partial}{\partial x_{j}}\left(\rho U_{j} H\right)=\frac{\partial}{\partial x_{j}}\left(\lambda \frac{\partial T}{\partial x_{j}}+\sum_{i}^{N_{c}} \Gamma_{i} h_{i} \frac{\partial Y_{i}}{\partial x_{j}}+\frac{\mu_{t}}{P_{r_{t}}} \frac{\partial h}{\partial x_{j}}\right)+S_{E} \quad$ Eq. 66 


\section{Multicomponent Energy Diffusion}

In the special case that all diffusivities are the same and equal to thermal conductivity divided by specific heat capacity:

\section{$\Gamma_{\mathrm{i}}=\Gamma=\frac{\lambda}{c_{\mathrm{p}}} \quad$ Eq. 67}

This holds when the Lewis number is unity for all components: $L e_{i}=\lambda /\left(c_{p} \Gamma_{i}\right)=1$ for turbulent flow assuming unity is usually just as good just as good as the common practice of using the unity Schmidt number. For $\mathrm{Le}_{\mathrm{i}}=1$ the energy equation simplifies exactly to the following:

$\frac{\partial}{\partial t}(\rho H)-\frac{\partial P}{\partial t}+\frac{\partial}{\partial x_{j}}\left(\rho U_{j} H\right)=\frac{\partial}{\partial x_{j}}\left[\left(\frac{\lambda}{c_{p}}+\frac{\mu_{t}}{P_{r_{t}}}\right) \frac{\partial h}{\partial x_{j}}\right]+S_{E} \quad$ Eq. 68

This equation has the advantage of having only a single diffusion term reducing the number of computations. When component dependent turbulent Schmidt numbers are specified then turbulent energy flux must be specified. This can be achieved by splitting turbulent fluctuation of enthalpy into its contributions from temperature, pressure, and component mass fractions:

\section{$\mathbf{h}^{\prime \prime} \approx \mathbf{C}_{\mathbf{p}} \mathbf{T}^{\prime \prime}+\frac{\partial \mathrm{h}}{\partial \mathbf{P}} \mathbf{P}^{\prime \prime}+\sum_{\mathrm{i}=\mathbf{1}}^{\mathrm{N}_{\mathrm{c}}} \mathbf{h}_{\mathbf{i}} \mathbf{Y}_{\mathrm{i}}^{\prime \prime} \quad$ Eq. 69}

Using this transformation the turbulent energy flux can then be modeled using the eddy diffusivity model with turbulent Prandtl number for the temperature fluctuations plus the sum of the secondary enthalpy transport terms:

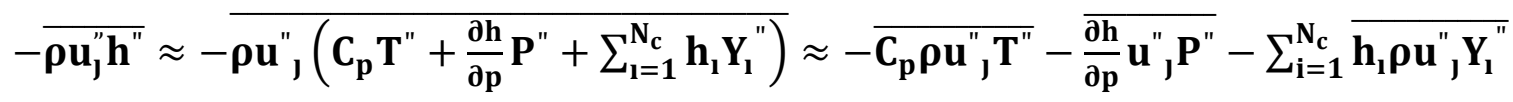

Eq. 70

$$
\approx \mathrm{C}_{\mathrm{p}} \frac{\mu_{\mathrm{t}}}{\mathrm{Pr}_{\mathrm{t}}} \cdot \frac{\partial \mathrm{T}}{\partial \mathrm{x}_{\mathrm{j}}}+\left(\frac{\partial \mathrm{h}}{\partial \mathrm{P}} \cdot \frac{\mu_{\mathrm{t}}}{\mathrm{Pr}_{\mathrm{t}}}\right) \frac{\partial \mathrm{P}}{\partial \mathrm{x}_{\mathrm{j}}}-\sum_{\mathrm{i}=1}^{\mathrm{N}_{\mathrm{c}}}\left(\mathrm{h}_{\mathrm{i}} \overline{\rho \mathrm{u}^{\prime \prime}, \mathrm{Y}_{1}^{\prime \prime}}\right) \quad \text { Eq. } 71
$$


Inserting the eddy diffusivity model for turbulent component mass fluxes yields the following model for the turbulent enthalpy flux:

$-\overline{\rho u_{j}^{\prime \prime} h^{\prime \prime}} \approx \mathbf{C}_{\mathbf{p}} \frac{\mu_{t}}{P_{r_{t}}} \cdot \frac{\partial T}{\partial x_{j}}+\left(\frac{\partial h}{\partial P} \cdot \frac{\mu_{t}}{P_{r_{t}}}\right) \frac{\partial P}{\partial x_{j}}+\sum_{i=1}^{N_{c}} h_{i}\left(\frac{\mu_{t}}{s_{c_{t}, i}} \cdot \frac{\partial Y_{i}}{\partial x_{j}}\right)$

Using these relations with the energy equation allows it to be rewritten for generalized turbulent component transport:

$\frac{\partial}{\partial t}(\rho H)-\frac{\partial P}{\partial t}+\frac{\partial}{\partial x_{j}}\left(\rho U_{j} H\right)=\frac{\partial}{\partial x_{j}}\left[\left(\lambda+C_{p} \frac{\mu_{t}}{P r_{t}}\right) \frac{\partial T}{\partial x_{j}}+\left(\frac{\partial h}{\partial P} \cdot \frac{\mu_{t}}{P r_{t}}\right) \frac{\partial P}{\partial x_{j}}+\sum_{i=1}^{N_{c}} h_{i}\left(\Gamma_{i} \frac{\partial Y_{i}}{\partial x_{j}}+\right.\right.$

$\left.\left.\overline{\rho u^{\prime \prime} Y_{1}^{\prime \prime}}\right)\right]+S_{E} \quad$ Eq. 73

If the eddy diffusivity assumption is being used to model the turbulent mass flux for each component then the equation becomes:

$\frac{\partial}{\partial t}(\rho H)-\frac{\partial P}{\partial t}+\frac{\partial}{\partial x_{j}}\left(\rho U_{j} H\right)=\frac{\partial}{\partial x_{j}}\left[\left(\lambda+C_{p} \frac{\mu_{t}}{P_{r_{t}}}\right) \frac{\partial T}{\partial x_{j}}+\left(\frac{\partial h}{\partial P} \cdot \frac{\mu_{t}}{P_{r_{t}}}\right) \frac{\partial P}{\partial x_{j}}+\sum_{i=1}^{N_{c}} \mathbf{h}_{i}\left(\Gamma_{i}+\right.\right.$

$\left.\left.\frac{\mu_{t}}{s_{c_{t, i}}}\right) \frac{\partial Y_{i}}{\partial x_{j}}\right]+S_{E} \quad$ Eq. 74

\subsubsection{Additional Variables}

Transport Equations for Additional Variables

The general form of the transport equation for an additional variable is:

$\frac{\partial(\rho \varphi)}{\partial t}+\nabla \cdot(\rho U \varphi)=\nabla \cdot\left(\rho D_{\phi} \nabla_{\varphi}\right)+S_{\varphi} \quad$ Eq. 75

Where:

$\mathrm{U}$ is the fluid velocity in a fluid or porous domain or $U_{s}$ in the case of solid motion velocity with $U_{s}$ is the solid velocity

$\rho$ is the mixture density in mass per unit volume

$\Phi$ is the conserved quantity per unit volume, or the concentration 
$\varphi=\Phi / \rho$ is the conserved quantity per unit mass

$S_{\varphi}$ is a volumetric source term, with units of conserved quantity per unit time

$D_{\Phi}$ is the kinematic diffusivity for the scalar

For turbulent flow, this equation is Reynolds-averaged and becomes:

$\frac{\partial(\rho \varphi)}{\partial t}+\nabla \cdot(\rho U \varphi)=\nabla \cdot\left(\left(\rho D_{\Phi}+\frac{\mu_{t}}{S_{c_{t}}}\right) \nabla \varphi\right)+S_{\varphi} \quad$ Eq. 76

Where:

$S_{c t}$ is the turbulence Schmidt number

$\mu_{t}$ is the turbulence viscosity

\section{Diffusive Transport Equations for Additional Variables}

The general form of the diffusive transport equation for an additional variable (non-reacting scalar) is:

$\frac{\partial(\rho \varphi)}{\partial t}=\nabla \cdot\left(\rho D_{\Phi} \nabla_{\varphi}\right)+S_{\varphi} \quad$ Eq. 77

The variables are all the same as in the regular transport equation.

\section{Poisson Equations for Additional Variables}

The general form of the Poisson equation for an additional variable (non-reacting scalar) is:

$\nabla \cdot\left(\rho D_{\Phi} \nabla_{\varphi}\right)+S_{\varphi}=0 \quad$ Eq. 78

The variables are also the same as the past two equations.

\section{Momentum Sources}

A momentum source is implemented as a force per unit volume acting on the fluid(s). Momentum sources can be used to model isotropic losses in porous regions, directional losses in porous regions among other processes. 


\section{Isotropic Loss Model}

The momentum loss through an isotropic porous region can be formulated using permeability and loss coefficients as follows:

$S_{M, x}=-\frac{\mu}{K_{\text {perm }}} U_{x}-K_{\text {loss }} \frac{\rho}{2}|\mathbf{U}| U_{x} \quad$ Eq. 79

$S_{M, y}=-\frac{\mu}{K_{\text {perm }}} U_{y}-K_{\text {loss }} \frac{\rho}{2}|U| U_{y} \quad$ Eq. 80

$S_{\mathrm{M}, \mathrm{z}}=-\frac{\mu}{K_{\text {perm }}} \mathbf{U}_{\mathrm{z}}-\mathrm{K}_{\text {loss }} \frac{\rho}{2}|\mathbf{U}| \mathbf{U}_{\mathrm{z}} \quad$ Eq. 81

Where $\mathrm{K}_{\text {perm }}$ is the permeability coefficient and $\mathrm{K}_{\text {loss }}$ is the quadratic loss coefficient. The linear component of this source represents the viscous losses and the quadratic term represents inertial losses. Alternatively the source may be formulated using linear and quadratic resistance coefficients substituting $C_{R 1}$ and $C_{R 2}$ for the others as follows:
$\mathrm{C}_{\mathrm{R} 1}=\frac{\mu}{\mathrm{K}_{\mathrm{perm}}}$
Eq. 82
$\mathrm{C}_{\mathrm{R} 2}=\mathrm{K}_{\text {loss }} \frac{\rho}{2}$
Eq. 83

\subsubsection{Directional Loss Model}

The momentum source through an anisotropic porous region may be modeled using the directional loss model. With this model the streamwise direction must be specified. If the streamwise coordinate system was $\left(x^{\prime}, y^{\prime}, z^{\prime}\right)$ such that the $x^{\prime}-$ axis is aligned with the streamwise direction and the y' and z' axes lie on the transverse plane then the momentum losses in these directions are:

$$
\begin{array}{ll}
S_{\mathrm{M}, \mathrm{x}^{\prime}}=-\frac{\mu}{K_{\text {perm }}^{S}} U_{\mathbf{x}^{\prime}}-K_{\text {loss }}^{S} \frac{\rho}{2}|\mathbf{U}| \mathbf{U}_{\mathbf{x}^{\prime}} & \text { Eq. } 84 \\
S_{\mathrm{M}, \mathrm{y}^{\prime}}=-\frac{\mu}{K_{\text {perm }}^{\mathrm{T}}} \mathbf{U}_{\mathbf{y}^{\prime}}-K_{\text {loss }}^{\mathrm{T}} \frac{\rho}{2}|\mathbf{U}| \mathbf{U}_{\mathbf{y}^{\prime}} & \text { Eq. } 85
\end{array}
$$


$\mathbf{S}_{\mathrm{M}, \mathrm{z}^{\prime}}=-\frac{\mu}{\mathbf{K}_{\mathrm{perm}}^{\mathrm{T}}} \mathbf{U}_{\mathrm{z}^{\prime}}-\mathbf{K}_{\text {loss }}^{\mathrm{T}} \frac{\rho}{2}|\mathbf{U}| \mathbf{U}_{\mathrm{z}^{\prime}} \quad$ Eq. 86

In this case there is an individual permeability and loss coefficient term for both the stream-wise and transverse directions. Just as in the isotropic model these may be expressed in terms of the linear and quadratic resistance coefficients.

\subsubsection{General Momentum Sources}

The general momentum source is available in the instance that there sources are not covered by the isotropic or directional loss models. A source for each direction is given by:

$S_{M, x}=S_{\text {spec, } x} i \quad$ Eq. 87

$S_{M, y}=S_{s p e c, y} j \quad$ Eq. 88

$S_{M, z}=S_{\text {spec,z }} k \quad$ Eq. 89

Where $\mathrm{S}_{\mathrm{spec}}$ are the specific momentum components. Additionally a representative scalar linearization coefficient based on the derivate

$\frac{\partial \overrightarrow{S_{m}}}{\partial \vec{U}} \quad$ Eq. 90

May be specified to provide robust convergence when a general momentum source is present.

\subsubsection{Immersed Solids}

An immersed solid is a rigid compact body capable of moving within a fluid domain. In order to capture the movement of the immersed body, the fluid volume is forced to move with the solid by applying a source term to the momentum equation.

$S_{x}=-\alpha \beta C\left(\mathbf{U}_{x}-\mathbf{U}_{\mathbf{x}}^{F}\right)$ 
$S_{x}=-\alpha \beta C\left(U_{x}-U_{x}^{F}\right)$

$S_{x}=-\alpha \beta C\left(\mathbf{U}_{x}-\mathbf{U}_{x}^{F}\right)$

Where $\mathbf{U}_{\mathbf{x}}, \mathbf{U}_{\mathbf{y}}, \mathbf{U}_{\mathbf{z}}$ are the three components of fluid velocity.

$\mathbf{U}_{\mathbf{x}}^{\mathrm{F}}, \mathbf{U}_{\mathbf{y}}^{\mathrm{F}}, \mathbf{U}_{\mathbf{z}}^{\mathrm{F}}$ are the components of forcing velocity due to the immersed solid.

$-\mathbf{C}$ is a large number, and is evaluated as the average of the three diagonal coefficients in the momentum equation.

$\boldsymbol{\alpha}$ is the Momentum Force Scaling Factor.

\subsection{CFD usage for emerging technologies in Heart Valve Research}

Driessen et al. (Driessen et al., 2007) employed a mathematical model to study the mechanical properties of a TEHV. Engelmayer et al. (Engelmayr et al., 2008) utilized GAMBIT® (v2.1) and FLUENT ® (v6.2) ( Ansys, Canonsburg, PA) to study flow behavior through a novel bioreactor for heart valve tissue engineering. Computational studies on the flow physics on and around the housed bioreactor specimens (Engelmayr et al., 2008) were subsequently evaluated further by Ramaswamy et al. (Ramaswamy et al., 2010). The commercially available software FLUENT $®$ (Ansys, Canonsburg, PA) was employed on a structured grid composed of 1.43 million hexahedral elements and 1.54 million nodes. They hypothesized that the oscillatory shear stresses that were created during specimen, valve-like bending were involved in engineered heart valve tissue formation (Ramaswamy et al., 2010).

Ramaswamy et al. (Ramaswamy et al., 2014) also developed a u-shaped novel bioreactor. Its design permitted the in vitro recreation of physiologically relevant shear stress magnitudes. 


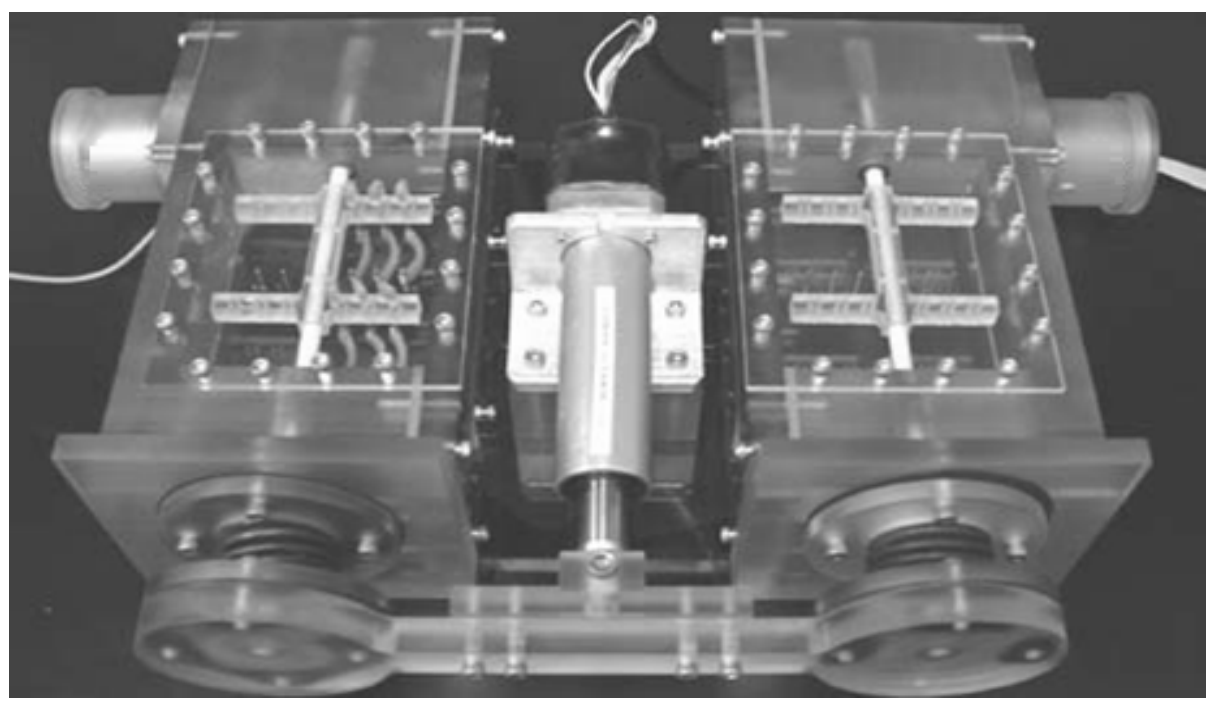

Figure 3. Custom made mechanical conditioning bioreactor for HVTE studies (Engelmayr et al., 2006). Modes: Flow, stretch, flexure.
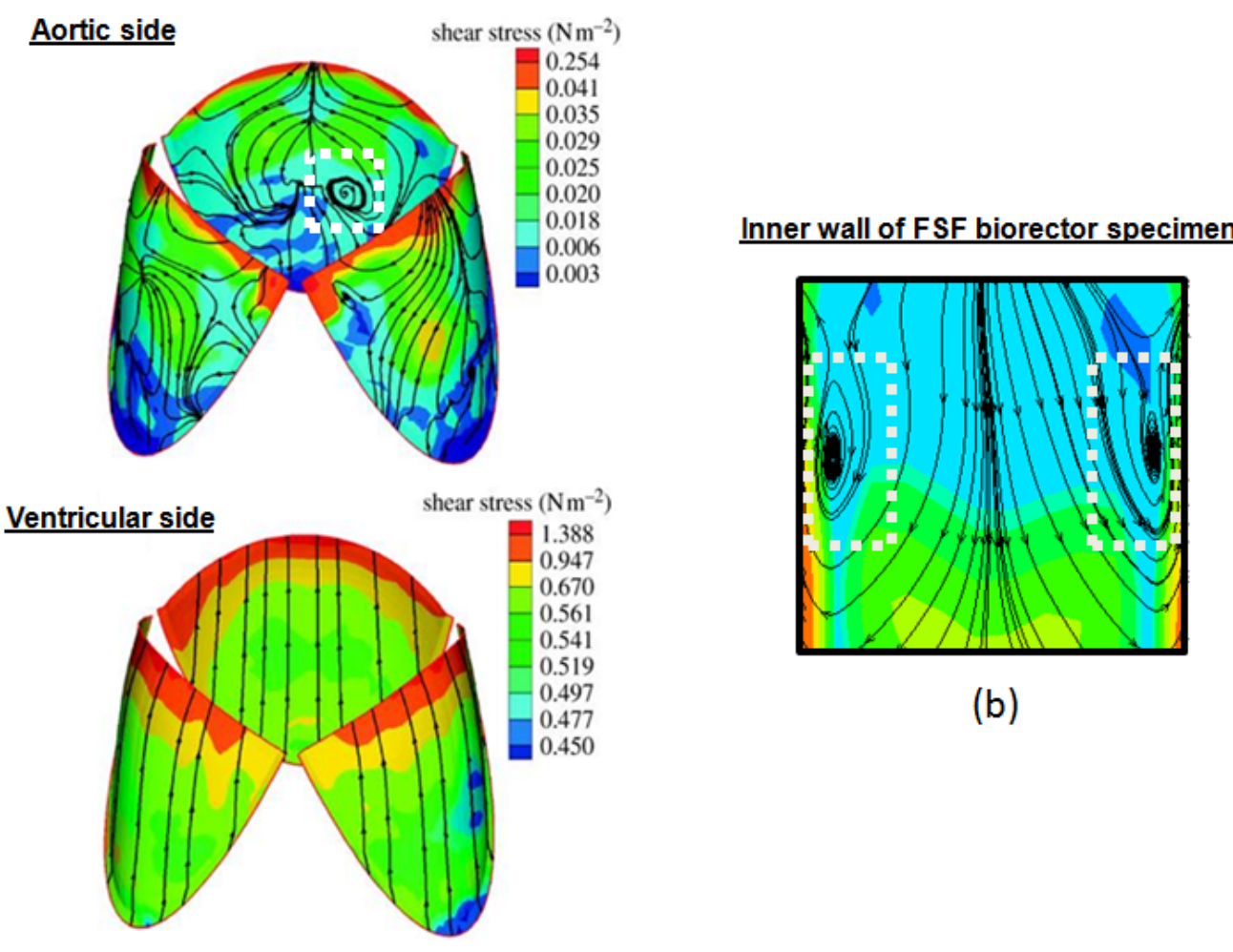

(b)

(a)

Figure 4. Computational fluid dynamic simulations on native heart valve (a) and rectangular (b) Geometries (Ramaswamy et al., 2010). 


\subsection{Recent Advances in CFD Applications for Heart Valve Research}

\subsubsection{Imaging to Meshing to CFD}

Blood flow physics that occur in the neighborhood of native or prosthetic valves can be a key indicator of the functionality of the valve (Sotiropoulos and Borazjani, 2009). It can reveal disease in native valves (Ryan et al., 2006;

Schlosshan et al., 2011) and performance issues in the case of prosthetic ones (Sotiropoulos and Borazjani, 2009). In order to develop a strong-accurate CFD model, the incorporation of these flow physics as boundary or initial conditions is paramount (Le and Sotiropoulos, 2013; Wong et al., 2012). Equally important is the usage of geometry structures that correctly depict valve and surrounding tissues in vivo (Le and Sotiropoulos, 2013). This represents a key challenge due to the light scattering nature of blood which excludes the usage of some imaging techniques (Sengupta et al., 2012a). It is also important to be able to compare computational results with experimental data, and this can be achieved only if the valvular flow physics can be sufficiently captured in vivo.

Doppler echocardiography and cardiac magnetic resonance (CMR) have typically been used to obtain mainly unidirectional velocity profiles (2D) (Cavalcante et al., 2012; Sengupta et al., 2012a). More advanced versions such as the 4D flow CMR can provide temporal and 3D spatial information (Sengupta et al., 2012a) with resolution in the order of $50 \mathrm{~ms}$ and $3 \mathrm{~mm} 3$, respectively (Sengupta et al., 2012a). Color Doppler echocardiography has been used to observe abnormal flow patterns and superimposed turbulence (Ryan et al., 2006; Schlosshan et al., 2011). It does so via application of an ultrasonic beam that can 
be used to compute axial velocities (Leopaldi et al., 2012; Ryan et al., 2006). It can also provide radial velocities, but the integration of both radial and axial velocities, necessary for obtaining the true flow field, is not usually obtained in routine clinical management (Sengupta et al., 2012a).

Particle image velocimetry (PIV) is a popular technique for the characterization of the flow field through heart valves and chambers (Sengupta et al., 2012b). It is capable of capturing a highly physiologically similar flow field by using tiny particles that light up in response to the presence of a laser light. PIV does not track the individual particle displacement; it tracks the alignments of the particles as a group (Lim et al., 1998). Imaging technologies such as computer tomography (CT) scans, ultrasound and MRI can help in the virtual geometry reconstruction of cardiovascular component such as heart valves, chambers, and vessels (De Bartolo et al., 2012; Lantz and Karlsson, 2012; Le and Sotiropoulos, 2013; Wong et al., 2012). For instance, CT consists of taking multiple 2D cross section images that can be later stacked together for $3 \mathrm{D}$ reconstruction (Sengupta et al., 2012b). Presently, the accuracy and acceptability of CFD models greatly rely on these types of imaging techniques, which are capable of acquiring extensive 2D data that can be later processed to reconstruct $3 \mathrm{D}$ structures (Le and Sotiropoulos, 2013).

The advancement in imaging technologies, computational capabilities, better understanding of electrophysiology of the heart and its interactions with neighboring tissues and organs have infused new hope for the development of a virtual model that can closely resemble that of the heart and its valves 
(Cavalcante et al., 2012; De Bartolo et al., 2012; Le and Sotiropoulos, 2013). Meshing geometries with high density elements and nodes can be promptly accomplished through super computers (Haj-Ali et al., 2008; Kleinstreuer, 2006; Salinas et al., 2014a; Salinas et al., 2014b). Geometry re-meshing can be triggered by setting tolerance levels for specific parameters such as element volume or element angle deformation thereby delaying negative volume sectors which will cause computational errors that will terminate the iteration process without yielding any results (Ansys, 2014). Parallel processing with state of the art processors makes the iterative solution process more efficient. It is a common practice to set the number of iterations in the thousands. It also helps to achieve academic and research standard convergence criteria for continuity and momentum equations (Ramaswamy et al., 2010; Salinas et al., 2014a; Salinas et al., 2014b). We outline a few of the most up-to-date efforts in heart valve computational fluid modeling that were able to leverage accurate geometric reconstructions derived from original medical imaging datasets. We include specific case studies where appropriate (labeled with a case number) when visual illustrations are also provided. Case \# 1: Le and Sotiropoulos (Le and Sotiropoulos, 2013) employed the curvilinear immersed boundary method and developed a FSI model of a left ventricle and a mechanical heart valve. The 3D reconstruction of the ventricle geometry was obtained by merging 2D MRI images from a healthy individual. The results were found to be in good agreement with clinical observations. This work revealed complex valve leaflet kinematics and emphasized the importance of patient specific simulations for 
heart valve prosthesis and other cardiac devices. In Figure 5, it can be seen that there was the formation of coherent structures at different time points during the cardiac cycle. Coherent structures were well developed zones within the flow that exhibited a characteristic flow pattern such as vortices. Studying the formation and transient life of such structures could help in understanding damage to blood cells as well as prosthetic valve dynamics.

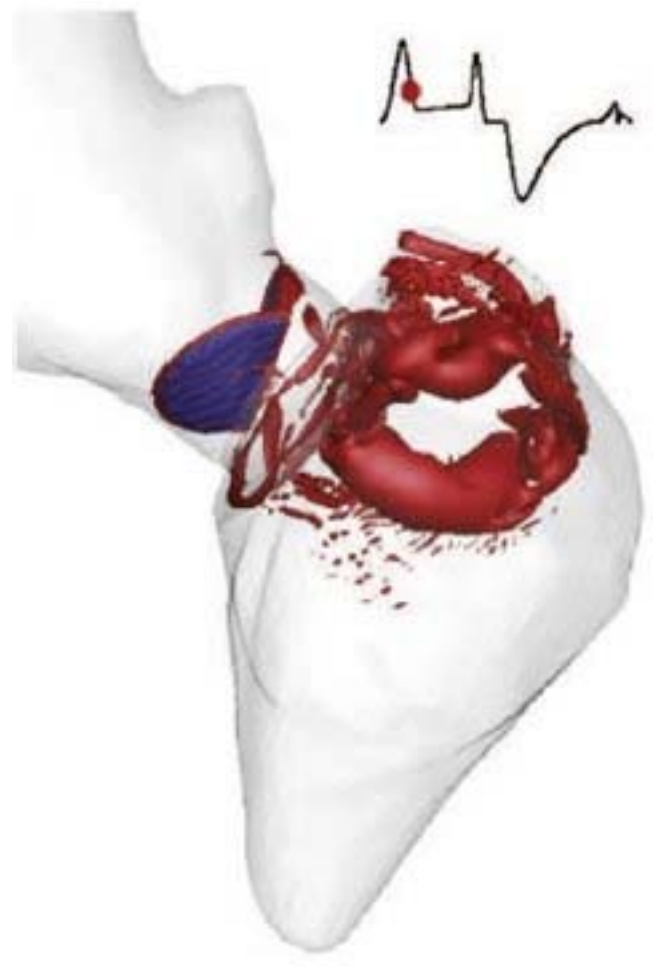

(a) E wave

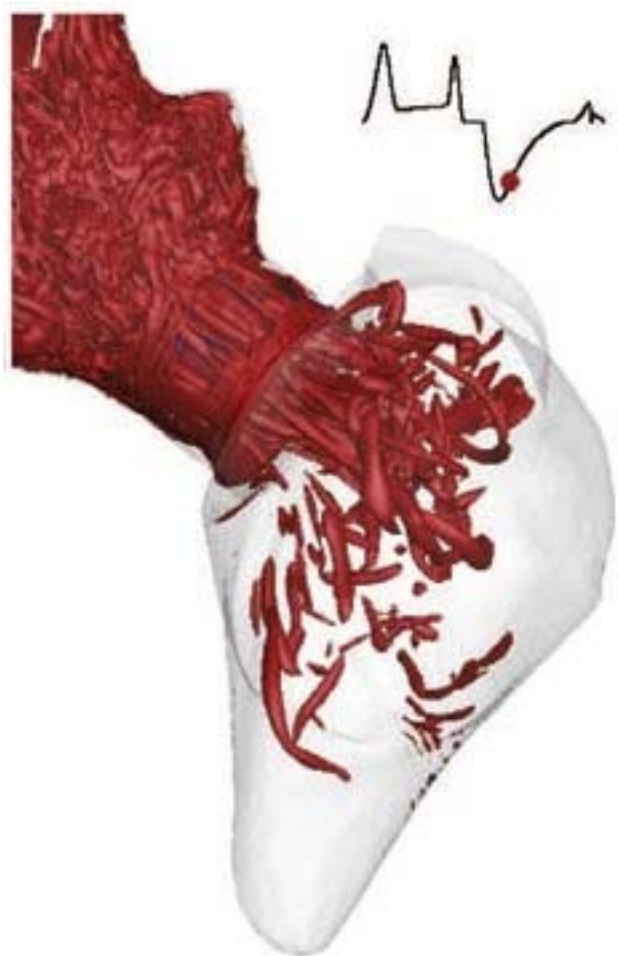

(b) End systole

Figure 5. The formation of coherent structures inside the left ventricular chamber during diastole in (a). The small scale structures are advected into the aorta during systole in (d). The red dot in the inset shows the time instance in the cardiac cycle. (From Le TB, Sotiropoulos F. Fluid-structure interaction of an aortic heart valve prosthesis driven by an animated anatomic left ventricle. $J$ Comput Phys. 2013(244):41-62. With permission from Elsevier)

Pellicioni et al. (Pelliccioni et al., 2007) utilized the Lattice-Boltzman (LB) method in a flow simulation past a mechanical valve in the aortic position. Other 
efforts that have utilized the same approach include the work of Krafczyk et al. (Krafczyk et al., 1998; Krafczyk et al., 2001). These efforts showed that the LB method was capable of predicting blood flow physics accurately.

Case \# 2: Shahriari et al. (Shahriari et al., 2012) developed a model for a bi-leaflet mechanical valve flow study. The model employed a smoothed particle hydrodynamics approach which is a mesh free approach. This study was the first of its kind to use this technique in the area of heart valve research and was originally developed for the astrophysics arena. Essentially, the fluid is represented by interacting particles that obey the Navier-Stokes equation using an interpolation function. The particles can move freely in the reference frame with a Lagragian configuration. The trajectory of the particles was used to correlate flow patterns with damage to blood components. In addition, large recirculation regions were found to be present in the dysfunctional BMHVs as shown in Figure 6. 

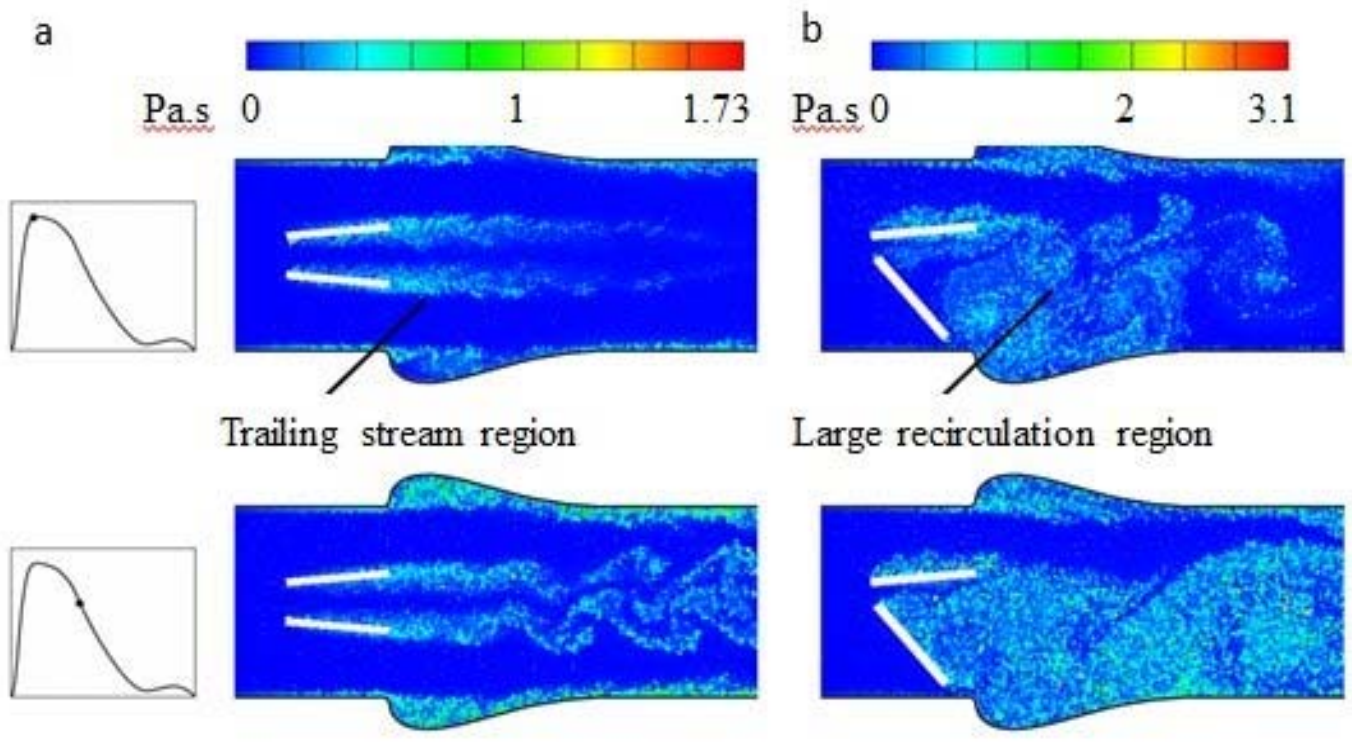

Large recirculation region
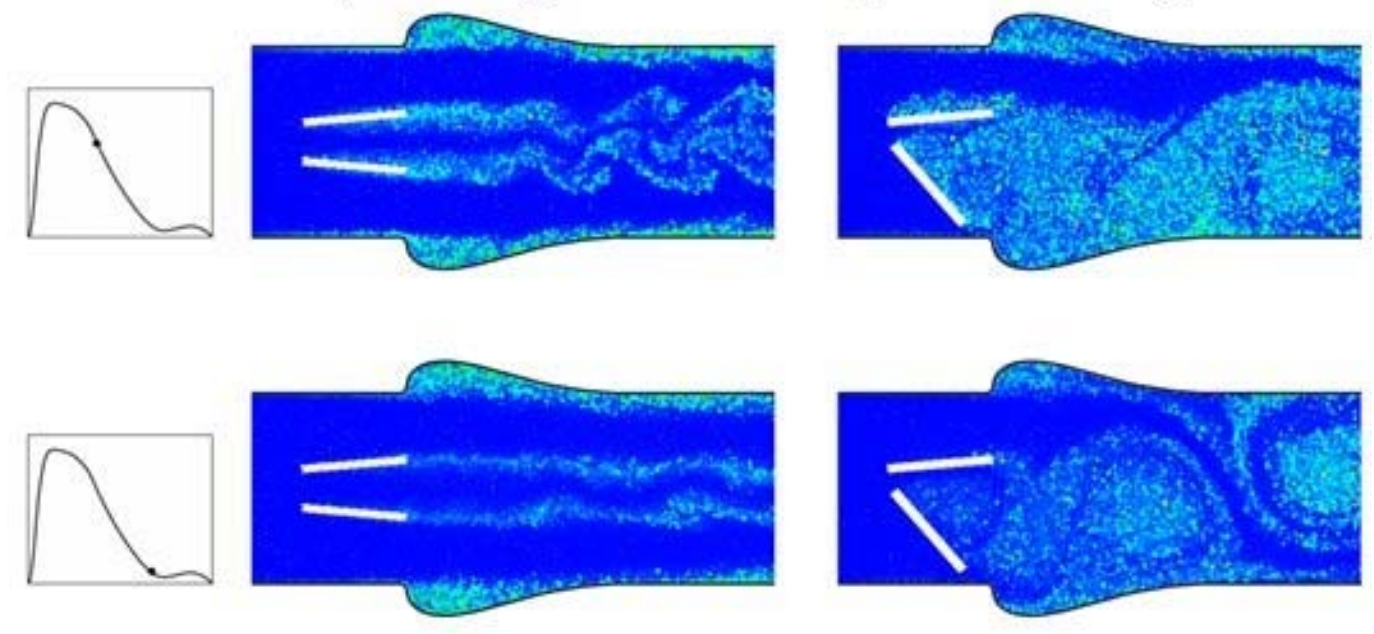

Figure 6. The patterns associated with accumulative shear stress applied on particles passing through: (a) a normal BMHV and (b) a dysfunctional BMHV.(From Shahriari S, Maleki H, Hassan I, Kadem L. Evaluation of shear stress accumulation on blood components in normal and dysfunctional bileaflet mechanical heart valves using smoothed particle hydrodynamics. J Biomech. 2012;45(15):2637-2644. With permission from Elsevier)

Case \# 3: Borazjani(Borazjani, 2013) developed a three-dimensional FSI model for both mechanical and bio-prosthetic valves. He used a Fung model for the soft tissue and validated his results with experimental and numerical heart valve tissue data. The mechanical results were validated using flow measurements from a mechanical heart valve. Figure 7, 8 and 9 show the comparison of shear stress, structure formation and the non-dimensional second Piola-Kirchhoff stress. It can be seen that shear stress acting on blood 
components was higher in the mechanical valve. Other efforts have shown a correlation between higher shear stress and thrombus nucleation(Banerjee, 2013; De Bartolo et al., 2012). The non-dimensional second Piola-Kirchhoff stress was used to identify the points of maximum stress which found to occur when flow pushed the valve toward the open position.
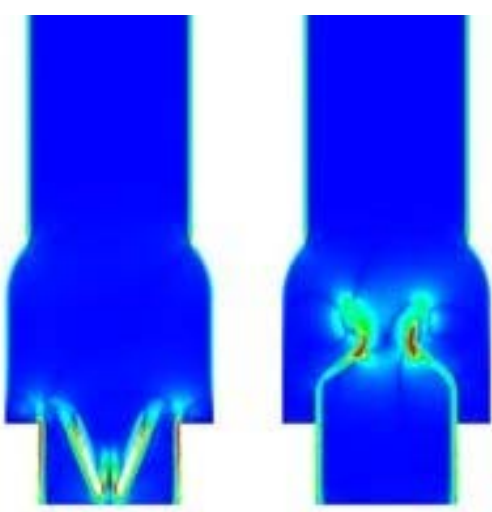

(a)

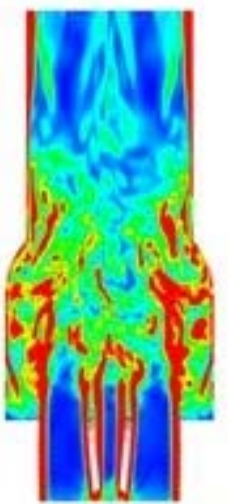

(c)

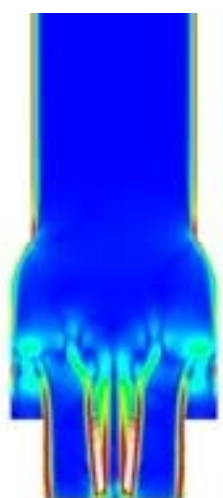

Shear $(\mathrm{Pa})$

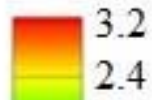

1.6

0.8

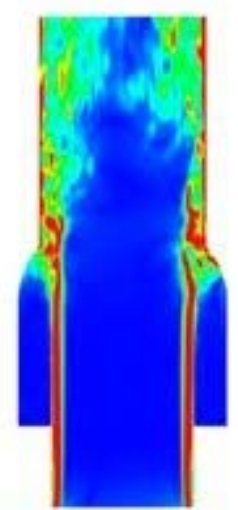

0

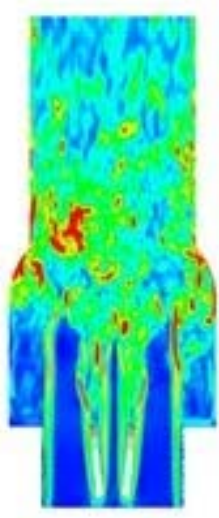

(d)

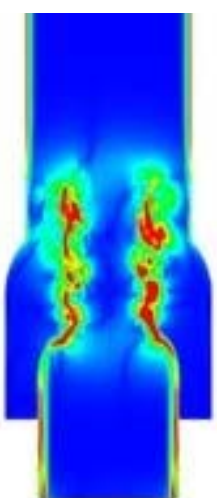

(b)

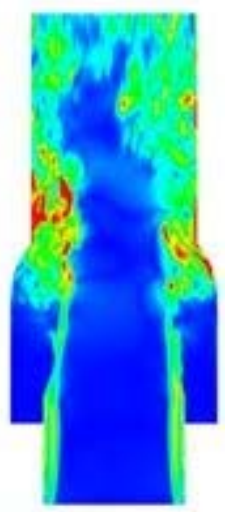

Figure 7. FSI simulations with peak $\operatorname{Re}=6000$ : Comparison of instantaneous local maximum shear sloc contours over the midplane of the valve for an MHV vs. BHV implanted in a straight aorta at time $t=$ (a) 52 , (b) 93 , (c) 186 , and (d) $334 \mathrm{~ms}$ within the cardiac cycle. (From Borazjani I. Fluid-structure interaction, immersed boundary-finite element method simulations of bio-prosthetic heart valves. Comput Methods Appl Mech Eng. 2013;257(0):103-116. With permission from Elsevier) 

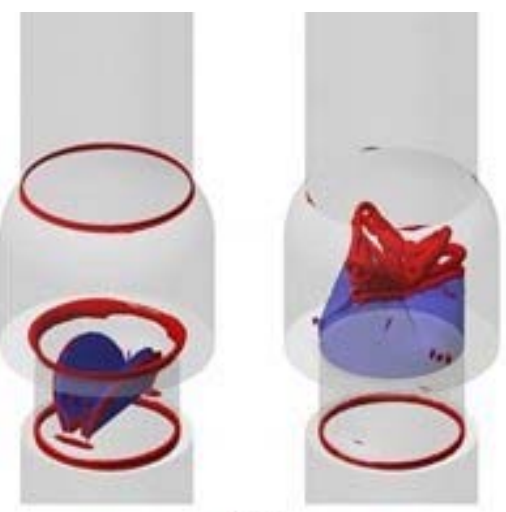

(a)
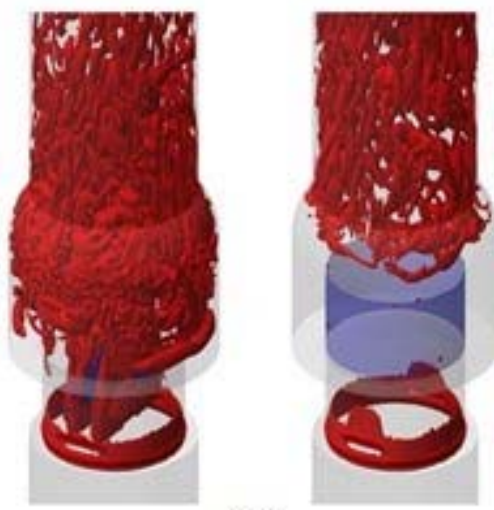

(c)
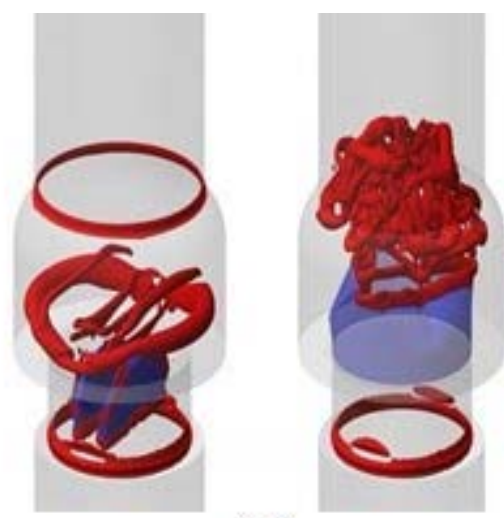

(b)
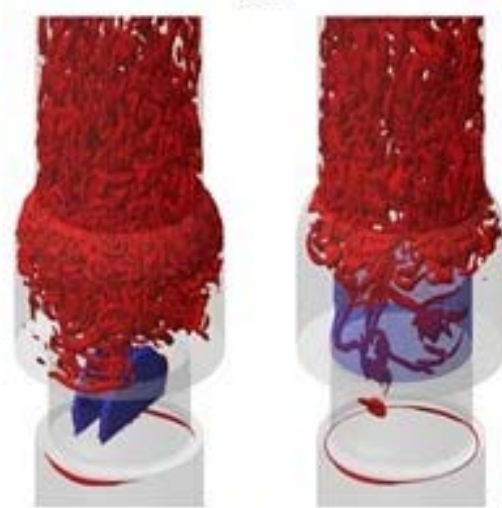

(d)

Figure 8. FSI simulations with peak $\operatorname{Re}=6000: 3 \mathrm{D}$ wake structure visualized by the iso-surfaces of q-criteria for a MHV vs. BHV implanted in a straight aorta at time $\mathrm{t}=$ (a) 52, (b) 93 , (c) 186, and (d) $334 \mathrm{~ms}$ within the cardiac cycle. (From Borazjani I. Fluid-structure interaction, immersed boundary-finite element method simulations of bio-prosthetic heart valves. Comput Methods Appl Mech Eng. 2013;257(0):103-116. With permission from Elsevier) 


\section{S Magnitude}

$\begin{array}{llll}0 & 1 & 2 & 3\end{array}$
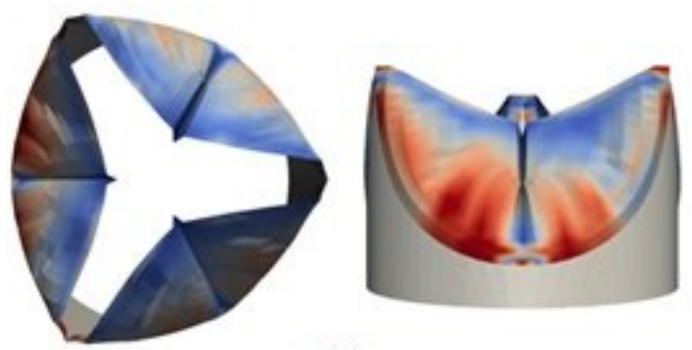

(a)

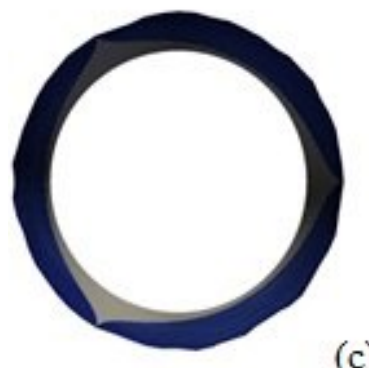

(c)
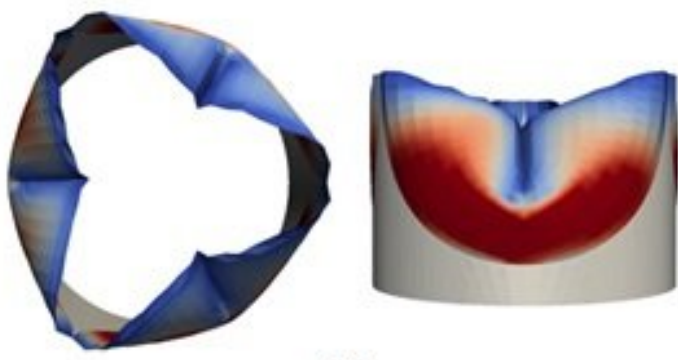

(b)

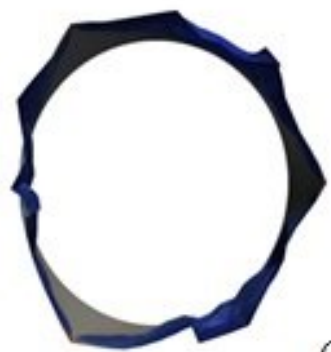

(d)

Figure 9. FSI simulations of BHV: The shape of the BHV and the magnitude of the non-dimensional second Piola-Kirchhoff stress $\mathrm{S}$ on the BHV at time instants $\mathrm{t}=$ (a) 52, (b) 93 , (c) 186, and (d) $334 \mathrm{~ms}$ within the cardiac cycle. (From Borazjani I. Fluid-structure interaction, immersed boundary-finite element method simulations of bio-prosthetic heart valves. Comput Methods Appl Mech Eng. 2013;257(0):103-116. With permission from Elsevier)

Case \# 4: We performed computational simulations of flow in a custom made bioreactor (Ramaswamy et al., 2014) capable of imparting physiologically relevant shear stresses onto rectangular scaffolds (Figure 10). The rationale for replicating native valve flow physics on rectangular surfaces was previously documented (Ramaswamy et al., 2010).

The movement of the samples was assumed to follow a parabolic profile during cyclic flexure deformation (Salinas et al., 2014a; Salinas et al., 2014b). In this study we wanted to observe the effect of flow pulsatility versus sample movement on oscillatory shear stresses (OSS). OSS has been shown to be 
important in the differentiation pathways of bone marrow derived stem cells (BMSCs) (Arnsdorf et al., 2009b; Arnsdorf et al., 2009a; Jacobs et al., 1998).

Our interests were in BMSC differentiation to support the heart valve phenotype, as it is a popular cell choice utilized in heart valve tissue engineering studies. We performed a quasi-static analysis on steady flow simulations at different sample bending positions. Pulsatile simulations were performed for fixed sample positions as described in Salinas et al.(Salinas et al., 2014a; Salinas et al., 2014b). All the simulations were carried out using CFX ${ }^{\circledR}$ (Ansys, Canonsburg, PA) software with parameters described in Figure 11.

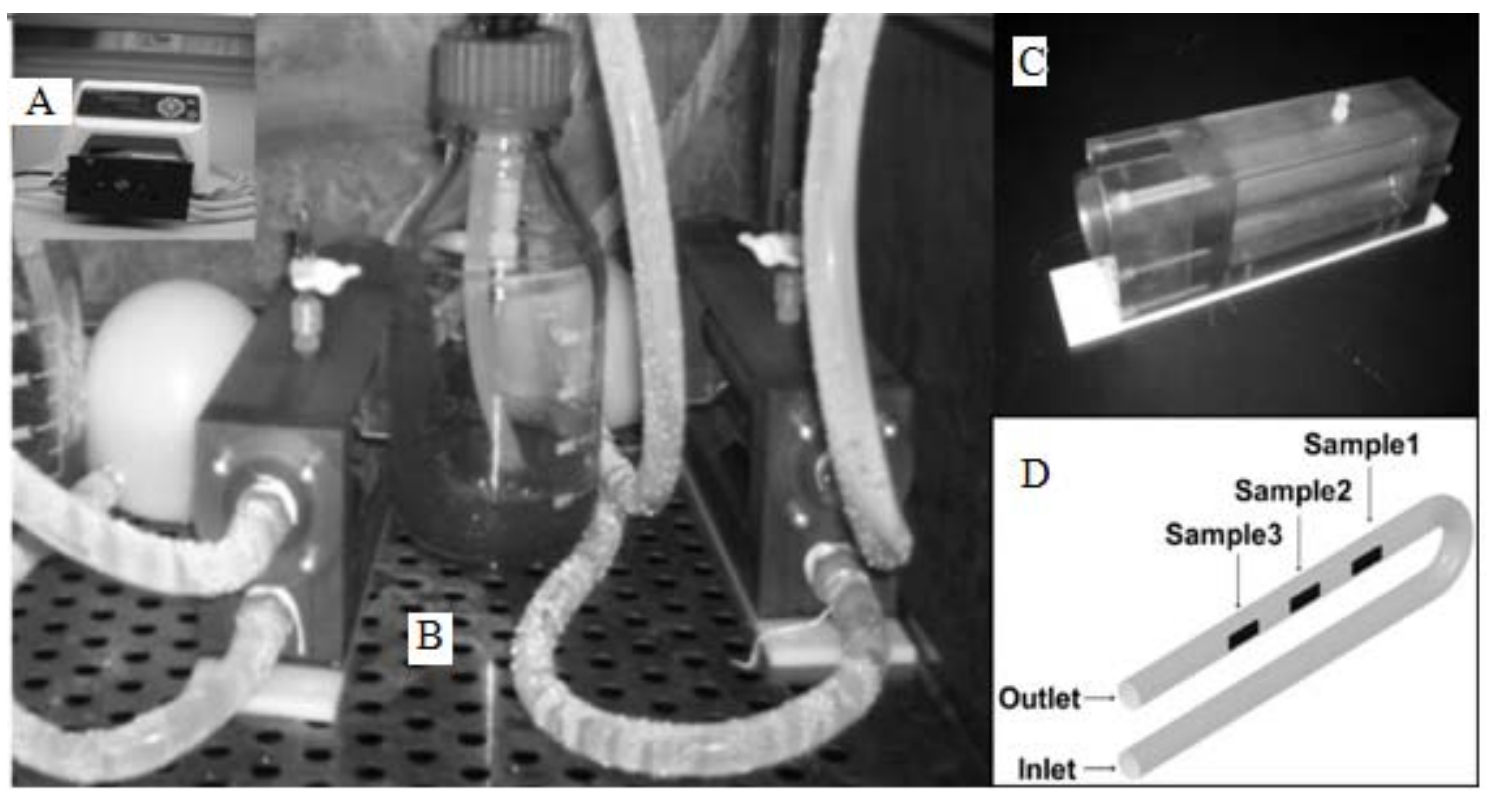

Figure 10. (A) Peristaltic pump used to induce flow through the FSF bioreactor. (B) FSF bioreactor set up. (C) Single FSF bioreactor chamber. (D) Bioreactor fluid domain showing the position of the three rectangular specimens. 


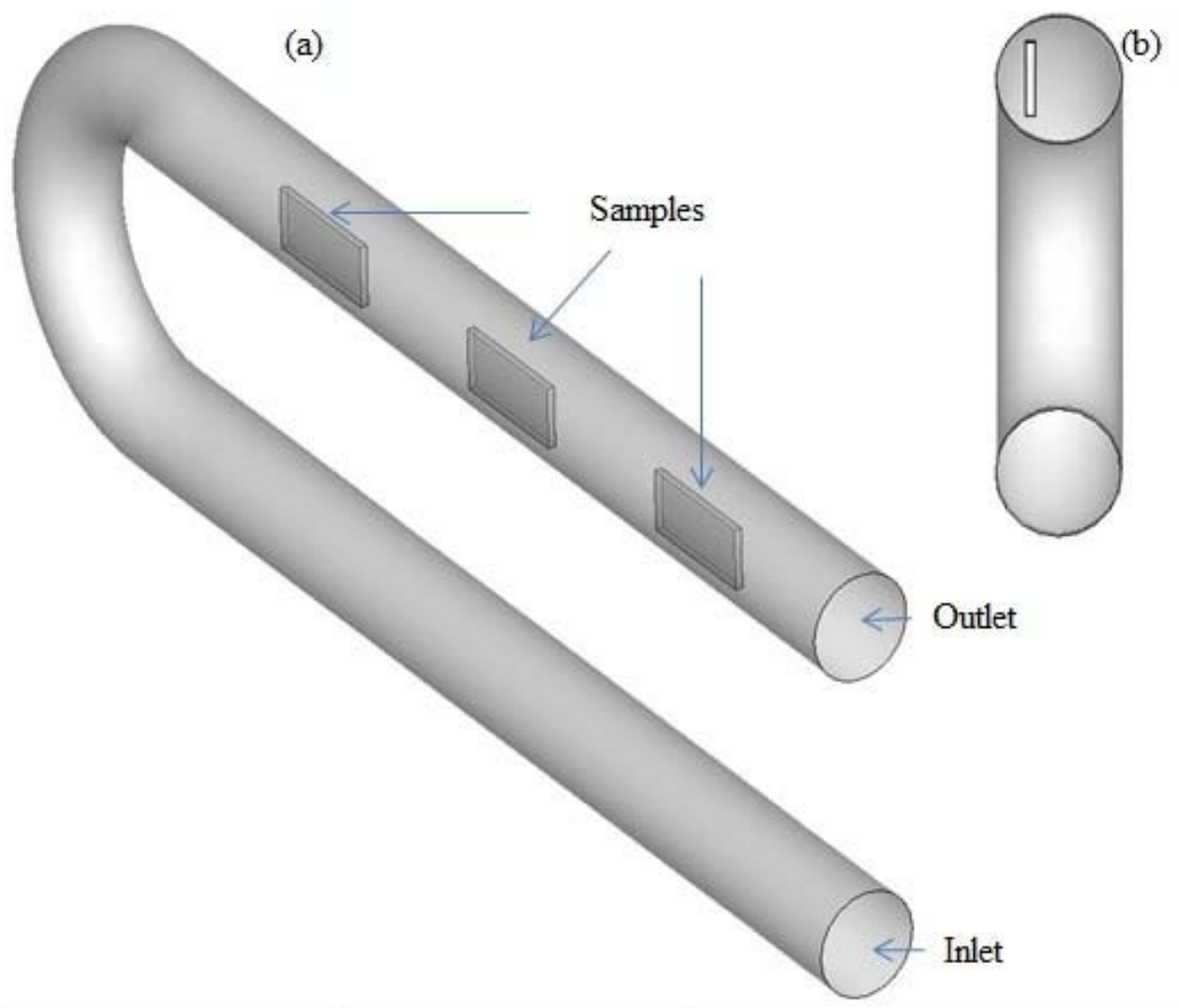

\begin{tabular}{||l|l|l||}
\hline \multicolumn{1}{||}{ CONDITION } & \multicolumn{1}{|c||}{ STEADY } & \multicolumn{1}{||}{ PULSA TILE } \\
\hline Flow & Laminar Flow & Laminar Flow \\
\hline Walls & $\begin{array}{l}\text { Walls-No slip } \\
\text { conditions }\end{array}$ & Walls-No slip conditions \\
\hline Inlet & $\begin{array}{l}\text { Plug velocity } \\
0.02 \mathrm{~m} / \mathrm{s}\end{array}$ & Velocity waveform ${ }^{6}$ \\
\hline Outlet & $\begin{array}{l}\text { Outlet-Opening } \\
\text { pressure }=0 \mathrm{~Pa}\end{array}$ & Outlet-Opening pressure $=0 \mathrm{~Pa}$ \\
\hline Convergence & $5 \times 10^{-2}$ & $5 \times 10^{-2}$ \\
\hline
\end{tabular}

Figure 11: (a) U-shaped bioreactor showing the three samples. (b) Front view of the bioreactor inlet, outlet and sample position. The Table indicates the parameters for steady and pulsatile simulations. 


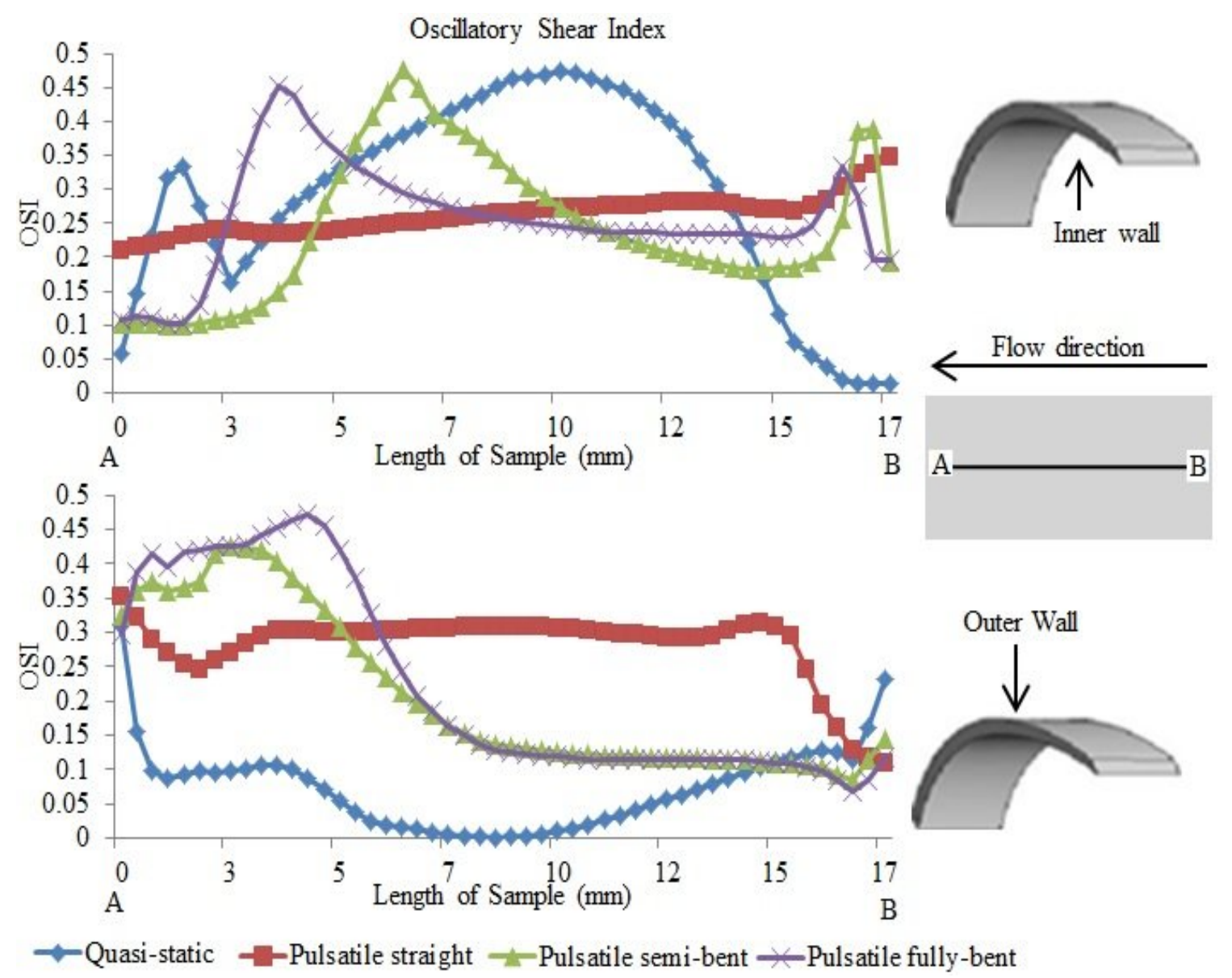

Figure 12: OSI distribution along the centerline of samples for (a): Inner wall and (b) Outer wall.Notice how the OSI variability was lowest along the inner and outer walls of straight samples $(\mathrm{N}=3)$ subjected to pulsatile flow. The average OSI in the inner surface was found to be $0.2627,0.2121,0.2396$, and 0.1009 for straight, semi-bent, fully-bent, and quasi-static, respectively. In that same order, the outer wall values were $0.2689,0.1859,0.2159$ and 0.2485 (Salinas et al., 2014a; Salinas et al., 2014b).

An oscillatory shear index (He and Ku, 1996) was used to quantify the OSS along the center line of the specimens. OSI was overall found to be the most uniform along both walls (inner and outer) for straight specimens that underwent pulsatile flow (Figure 12). In addition, mean OSI distribution was highest along both the inner and outer walls when specimens $(\mathrm{N}=3)$ were held fixed in a straight configuration (average specimen OSI $=0.26$, inner wall; 
average specimen $\mathrm{OSI}=0.27$, outer wall). Thus, these types of analyses illustrate an example of deriving insights from computational efforts without building complex CFD models (e.g. FSI), in aiding the design of experiments. In this case, the models guided the planning of maximizing OSS levels in BMSCseeded scaffolds uniformly. Subsequent evaluation of OSS-induced mechanobiology, could thus be accomplished in a clear-cut fashion, purely through a physiological pulsatile waveform, without the need for moving the specimens (Salinas et al., 2014a; Salinas et al., 2014b).

\subsection{Conclusions and Future Outlook}

The advent of new technologies such as tissue engineered heart valves and percutaneously-deployed valves are areas that are still relatively understudied using CFD. In this regard, several insights may be gained even from very straightforward modeling approaches (e.g. laminar flow, static models) provided that accurate depiction of the geometric features can be reproduced. On the other hand, the culmination of CFD research over the last several years has enabled streamlining of more advanced methodologies such as FSI-based approaches, to the extent that research groups that utilize their in-house developed code are able to simulate very complex dynamic states of heart valves in a highly efficient fashion. While these approaches are also available in commercial CFD packages, direct control of localized deformation states is still tedious. In the years to come, it is expected that these packages will begin to offer more flexibility in the prescription of mesh movement, thereby allowing for more direct control of the simulations by the end-user. 
Irrespective of whether the usage of an advanced CFD techniques become commonplace, or if several insights on the flow physics can be uncovered in emerging heart valve therapeutics using CFD, we expect that the findings resulting from CFD simulations in the heart valve research area will now, perhaps more than ever before, play a central role in the research or clinical process. This can be by way of, for example, enhancing the design features of a tissue engineered heart valve or a prosthetic valve, or alternatively, towards the evaluation of in vivo valve functionality, thereby guiding the surgeon in selecting the next course of treatment for the patient.

\section{CHAPTER 4: COMPUTER SIMULATIONS OF VALVE LIKE DEFORMATIONS}

\subsection{Introduction}

Tissue engineered heart valves (TEHVs) could potentially serve as a viable solution to congenital valve disease among pediatric patients who currently have extremely limited options (Fong et al., 2006; Kadner et al., 2002; Martinez et al., 2013; Schmidt et al., 2006). The primary appeal of TEHVs lies in their potential to integrate and grow with the patient, but in addition, TEHVs could potentially overcome current valve replacement barriers such as hemolysis, immune rejection, calcification, anticoagulation therapies and the need for multiple invasive surgeries (Chen et al., 2013; Hmiel, 2012; Leong et al., 2013; Munnelly et al., 2012; Nora et al., 2012; Syedain, 2009a).

As with native heart valves, the mechanical environment that TEHVs are exposed to will direct the nature of tissue remodeling that will occur. Studies have 
examined the remodeling responses of TEHVs using a variety of cell sources, both in vivo as well as in vitro in heart valve bioreactors, i.e., devices that impart valve-relevant mechanical stimuli (some combination of cyclic flexure, flow and cyclic stretch) to developing tissues. Many bioreactor-based studies have associated higher cell activity and tissue formation with fluid induced shear stresses (Kadner et al., 2002; Karim et al., 2006). A landmark study conducted by Engelmayr et al. (Engelmayr et al., 2006) utilized a bioreactor to condition rectangular polyglycolic acid /poly L-lactic acid (PGA/PLLA) non-woven fibrous scaffolds seeded with bone marrow stem cells (BMSCs). They reported that the combination of cyclic flexure and steady flow (flex-flow) greatly augmented engineered collagen formation when compared to steady flow or cyclic flexurealone. Interestingly, studies elsewhere described that oscillatory flow conditions mechanically regulate BMSCs (Arnsdorf et al., 2009b; Arnsdorf et al., 2009a; Li et al., 2004; Vermot et al., 2009). Indeed, oscillatory shear stress (OSS) has been shown to mediate BMSC proliferation and differentiation, (Arnsdorf et al., 2009b; Arnsdorf et al., 2009a). Specifically, Arnsdorf et al. (Arnsdorf et al., 2009b; Arnsdorf et al., 2009a) reported that OSS has the potential to promote osteogenic differentiation of BMSCs by activating key molecular pathways; meanwhile, Li et al. (Li et al., 2004) reported an increase in BMSC proliferation in addition to increases in intracellular $\mathrm{Ca}^{2+}$ signaling. Particularly relevant to heart valves, OSS was also found to directly regulate the gene klf2a which is required for normal valve development (Vermot et al., 2009). 
As a mechanical conditioning metric, steady flow on its own will serve to impart shear stresses, while cyclic flexure-alone will impart OSS on the surfaces of engineered tissue. It is only in flex-flow states that $\underline{\text { both }}$ shear stress magnitude and OSS will be augmented. Thus in the current study, our primary objective was to computationally quantify specimen surface: (1) shear stress magnitudes and (2) OSS present under, i) No flow, no flexure (control group) ii) steady flow-alone, iii) cyclic flexure-alone and iv) flex-flow states, and to subsequently co-relate them to previous experimental studies on engineered collagen production (Engelmayr et al., 2006) under these four corresponding culturing environments.

\subsection{Methods}

\subsubsection{Overview}

Computational Fluid dynamic (CFD) simulations of a flow-stretch-flexure (FSF) bioreactor available in our laboratory were performed. The physical description of the FSF bioreactor has been provided in detail previously (Boronyak et al., 2009; Ramaswamy et al., 2009a; Ramaswamy et al., 2013; Ramaswamy et al., 2008). The FSF bioreactor modeled in the present study is distinct from, but based on, the first FSF bioreactor, originally developed and reported by Engelmayr et al. (Engelmayr et al., 2008; Engelmayr et al., 2006). Briefly, the new device (Figure 13) can induce FSF stresses at the physiological range for up to three rectangular scaffolds per conditioning chamber without using viscosity additives. The complete set up comprises of four chambers. The sample movement is generated by an external actuator, and the flow is induced 
through a peristaltic pump. As mentioned, the individual and combined effects of steady flow and sample cyclic flexure on the regional flow physics of rectangular scaffolds were simulated in four cases.

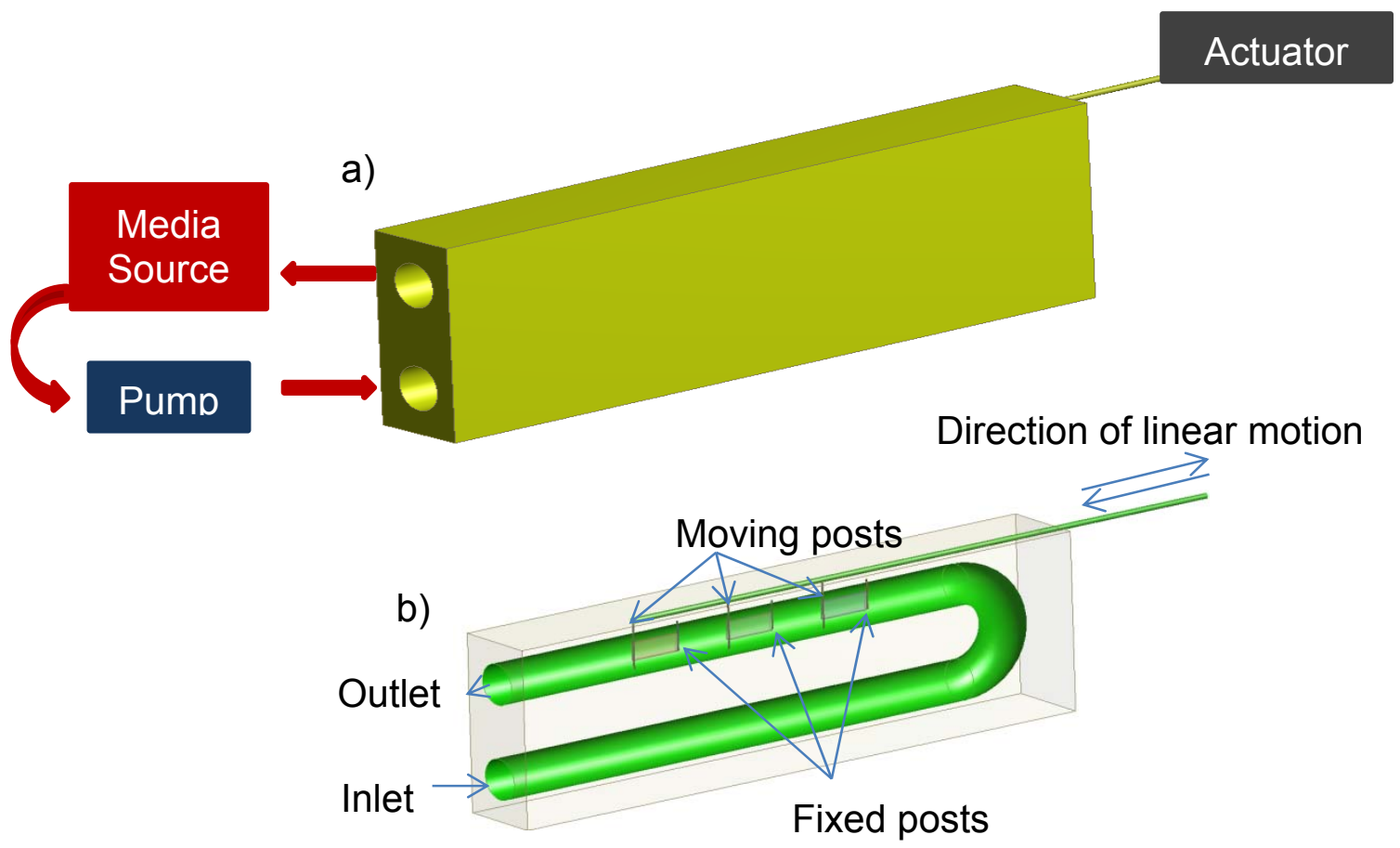

Figure 13: a) Shows a single bioreactor chamber connected to media source. The media is pumped into the bioreactor by a peristaltic pump (Boronyak et al., 2009; Ramaswamy et al., 2009a; Ramaswamy et al., 2009b; Ramaswamy et al., 2010; Ramaswamy et al., 2011; Ramaswamy et al., 2014). An external actuator attached to one end of the samples provides movement. b) Shows an inside look of the bioreactor. The chamber can hold three specimens. Each sample has one end fixed while the other is allowed to move linearly by means of the actuator.

\subsubsection{Mesh Construction, Simulation Set-up}

A 3D virtual geometry of the bioreactor's fluid domain was created (Design Modeler, Ansys Inc., Canonsburg, PA). The domain sample dimensions were 17 X $6.5 \times 1(\mathrm{~mm})$. A quadrilateral-element, structured mesh was subsequently constructed (Ansys Meshing, Ansys Inc., Canonsburg, PA). Since we were 
looking at the flow patterns-alone, the samples were suppressed after meshing and only the mesh for the fluid domain was imported into the CFD software for simulations (CFX 14.5 version, Ansys Inc, Canonsburg, PA). All simulations performed in this study imposed a Newtonian, viscous model, with laminar flow conditions and the following material parameters: the physical properties for bioreactor media were used with density $=1.01 \mathrm{~g} / \mathrm{cm}^{3}$ and dynamic Viscosity $=$ $1.27 \mathrm{cp}$. A zero relative-pressure condition was also applied at the outlet. Finally, a no slip boundary condition was prescribed to the bioreactor walls and also to the samples, for the cases where they did not move (no flow, no flexure and steady flow-alone). In all the CFD simulation runs, the models were analyzed once a convergence criteria of $1 \times 10^{-9}$ for the momentum and continuity equations were achieved. Simulations were conducted in a Hewlett Packard work station with intel(R) Xeon(R) CPU, x5550@2.67GHz (2 processors) with 16.0 GB installed memory and 64-bit windows 7 operating system. 


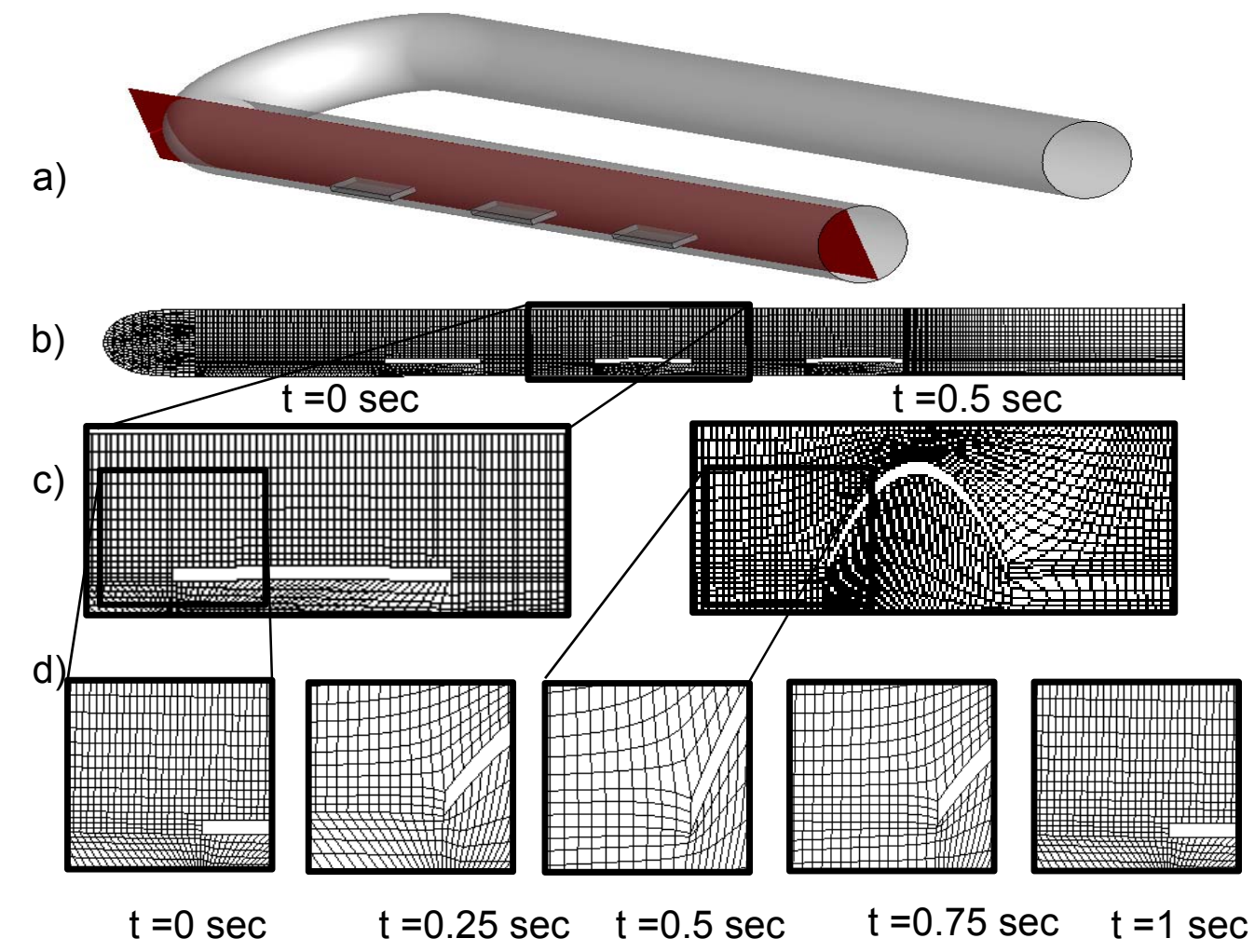

Figure 14: a) Depicts the entire fluid domain of the bioreactor chamber. Note the three scaffolds in a straight configuration and the plane (in red) at $x=0.075 \mathrm{~m}$ corresponding to the longitudinal center line of the samples. b) Cross section ( $x$ $=0.0175 \mathrm{~m}$ ) of the structured mesh (which consisted of 441,370 elements and 472,728 nodes) showing the distribution of quadrilateral elements and nodes. Note that the element density is higher around the samples and lower as it approaches the outlet. c) A close-up of the mesh around a single sample. The elements around the vicinity of the samples were aligned to conform to the flexing shape of the specimens. d) Close-up of the sample edge during $t=0 \mathrm{~s}, t$ $=0.25 \mathrm{~s}, \mathrm{t}=0.5 \mathrm{~s}, \mathrm{t}=0.75 \mathrm{~s}, \mathrm{t}=1 \mathrm{~s}$.

\subsubsection{Mesh Independence}

A similar simulation of the steady flow-alone case has been described in previous efforts (Salinas et al., 2014a; Salinas et al., 2014b). In that investigation, a relatively fine tetrahedral mesh consisting of $3.1 \times 10^{6}$ elements and $5.5 \times 10^{5}$ nodes was employed. The results revealed that the sample area-averaged shear stress was $0.031 \mathrm{~Pa}$. We continued optimizing our structured mesh density until 
a mesh consisting of 441,370 quadrilateral elements and 472,728 nodes (Figure 14) computed a corresponding average shear stress value that was found to be within $2 \%$ variability. This mesh was subsequently employed for all succeeding CFD simulations.

\subsubsection{Simulations involving non-moving samples (no flow, no flexure and steady flow-alone)}

In the control group, the samples were non-moving and an inlet flow velocity of negligible quantity was prescribed $\left(1 \times 10^{-7} \mathrm{~m} / \mathrm{s}\right)$. For the steady flow case, the samples were still held stationary and an inlet plug velocity profile of $0.022 \mathrm{~m} / \mathrm{s}$ was prescribed. Peak flow velocities in previous FSF bioreactor studies (Engelmayr et al., 2008) were determined to be in the order of $0.08 \mathrm{~m} / \mathrm{s}$. In the current computational study, we chose to prescribe an inlet velocity of $0.022 \mathrm{~m} / \mathrm{s}$ which represents a time-averaged blood velocity in the pulmonary artery (Lotz et al., 2002) and therefore is of particular physiological relevance to tissue engineered pulmonary valves. This prescribed velocity can readily be faciltiated by the flow source (Boronyak et al., 2009; Ramaswamy et al., 2009a; Ramaswamy et al., 2009b; Ramaswamy et al., 2010; Ramaswamy et al., 2011; Ramaswamy et al., 2014) of the FSF bioreactor modeled in this study. Three fixed specimen configurations were simulated in the steady flow-alone case: fully straight, semi-bent, and fully bent. These snapshot configurations corresponded to the initial, mid and final specimen shapes during the bending event. 


\subsubsection{Simulations involving moving samples (cyclic flexure-alone and flex- flow)}

In the CFD simulations for cyclic flexure-alone, the samples were allowed to bend, but the inlet velocity was set to zero. In the flex-flow simulation, the samples were flexed and the inlet plug velocity profile was set to $0.022 \mathrm{~m} / \mathrm{s}$. The set up and simulation of the flex-flow case is described in detail in (Ramaswamy et al., 2014). In brief, the samples were assumed to bend as a parabola. The construct deformed from a straight to bent configuration in $0.5 \mathrm{~s}$, and then from a fully bent to straight state in the next $0.5 \mathrm{~s}$. At a time step of $2 \mathrm{~ms}$, the samples were re-meshed to represent the change in sample shape while its movement was incorporated using an Arbitrary Lagrange Eularian (ALE) approach. Specifically, the ALE formulation uses a grid velocity which is coupled to the flow equations via the space conservation law. Details concerning the ALE formulation has been reported previously (Ansys, 2014; Lai and Przekwas, 1994; Ramaswamy et al., 2004). In brief, the continuity equation and the fully, time dependent, 3-D Navier-Stokes equations (inertial frame of reference) for an incompressible viscous fluid are:

$$
\begin{gathered}
\nabla \cdot \mathbf{V}=0 \quad \text { Eq. } 91 \\
\partial \mathrm{V} / \partial \mathrm{t}+(\mathbf{V} \cdot \nabla) \mathbf{V}=-1 / \rho(\nabla p)+v \nabla^{2} \mathbf{V} \quad \text { Eq. } 92
\end{gathered}
$$

Where, $\mathrm{V}$ is the velocity vector, $\mathrm{p}$ is the pressure, $\mathrm{t}$ is the time, $\rho$ is the fluid density, and $v$ is the kinematic viscosity. To permit motion, the ALE formulation 
introduces a grid velocity $\left(V_{g}\right)$ in equations 1 and 2 to impose an additional geometric constraint which is expressed in integral form as:

$$
\mathrm{d} / \mathrm{dt} \int_{\forall} \mathrm{d} \forall=\int_{\mathrm{s}} \mathrm{V}_{\mathrm{g}} \cdot \mathrm{ds}
$$

Note that $V_{g}$ is known a priori which in our case was identified as we have previously reported by us (Salinas et al., 2012; Salinas et al., 2014b), as follows: The FSF bioreactor specimens were assumed to take on a parabolic shape and fit to the equation of an arc, given by:

$$
\int_{-8.5}^{b} \sqrt{1+C x^{2}} d x=17 \mathrm{~mm} \quad \text { Eq. } 94
$$

Where $b$ is the new position of the moving post as it moves to the stationary post and $\mathrm{C}$ is the nonlinear parabolic constant. The specimen length was fixed at 17 mm. The constant, "C" was computed numerically (Mathcad® v.15, PTC, Needham, MA) over the 250 time steps. The continuity and momentum equations with $V_{g}$ incorporated can be re-expressed as:

$$
\begin{array}{r}
d / d t \int_{\forall} \rho d \forall+\int_{s} \rho\left(V-V_{g}\right) \cdot d s=0 \\
d / d t \int_{\forall} \rho V d \forall+\int_{s} \rho V\left(V-V_{g}\right) \cdot d s=\int_{s} \sigma_{i j} \cdot d s
\end{array}
$$

Where, $\forall$ is the arbitrarily moving control volume, $S$ is the surface of $\forall$, and $\sigma_{i j}$ is the second order stress tensor. With $V_{g}$ now known, we wrote a script interfaced to the main CFD software (CFX, Ansys Inc.), such that a numerical solution could be computed at a given temporal position, for the moving mesh.

In addition to satisfying the convergence criteria $\left(1 \times 10^{-9}\right)$ for the momentum and continuity equations, the final solution convergence was accomplished once the mesh displacement equations also satisfied the 
prescribed residual value of $1 \times 10^{-9}$. We note that for the dynamic simulations cyclic independence was achieved in the second cycle from which the results were analyzed, since a less than $5 \%$ difference was found when comparing the average specimen shear stress between the $2^{\text {nd }}$ and $3^{\text {rd }}$ cycles.

\subsubsection{Validation Studies}

\subsubsection{CFD Simulation of a moving indentation in a 2-D Channel}

Pedley and Stephanoff (Pedley and Stephanoff, 1985) conducted an in vitro experiment aimed at understanding the incompressible fluid flow through collapsible tubes such as arteries and veins. In their experiment, A $100 \mathrm{~mm} x$ $100 \mathrm{~mm}$ plate was pushed into a Poiseulle laminar flow, creating vortices. The total length and height of the channel was $410 \mathrm{~mm}$, and $10 \mathrm{~mm}$, respectively. Vortex characteristics were governed by the Strouhal and Reynolds numbers in addition to the degree of indentation.

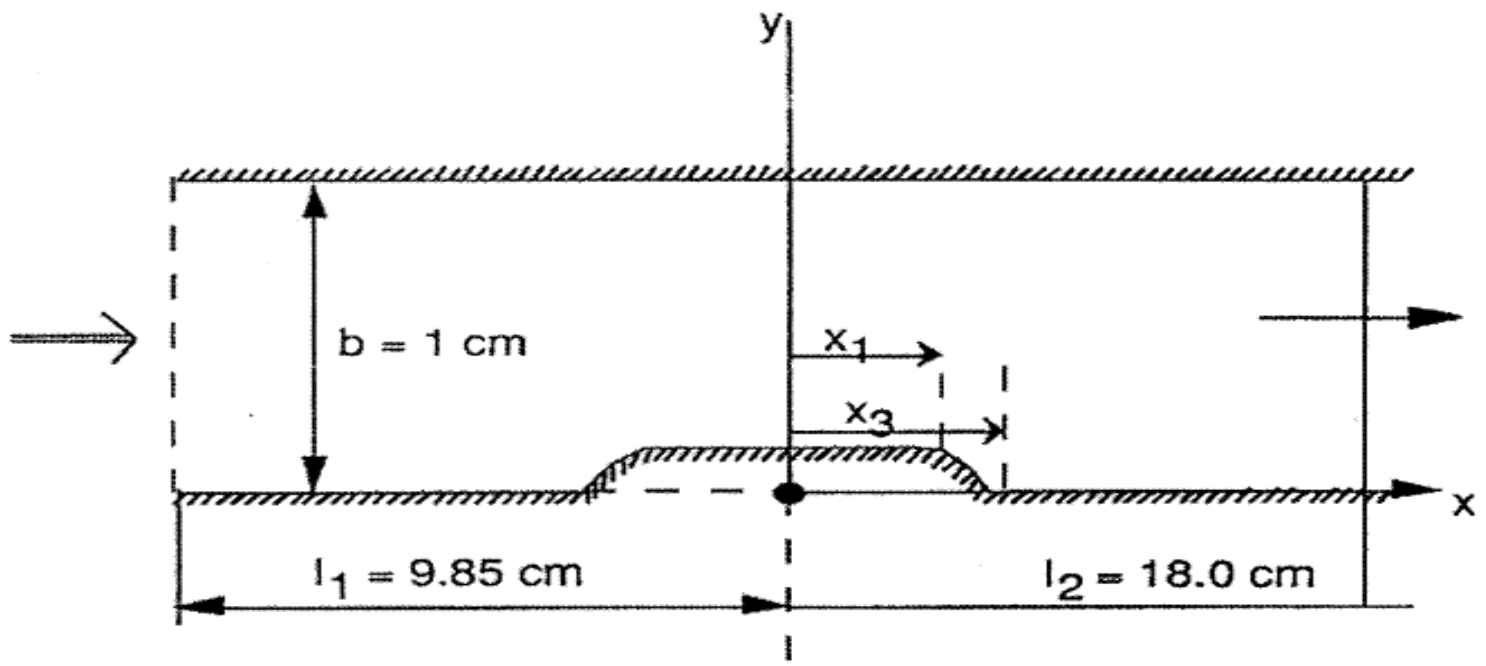

Figure 15. Schematic representation of the 2-D channel used in the experiment. Source: Lai Y.G., and Przekwas A.J., (1994). A Finite-Volume Method for Fluid Flow Simulations with Moving Boundaries. Computational Fluid Dynamics, Volume 2., pp. 19-40. 
The motion of the wall is governed by the following 2-D Cartesian equations:

$Y(x)=h \quad$ for $0<x<x_{1} \quad$ Eq. 97

$Y(x)=0.5 h\left\{1-\right.$ th $\left.\left[a\left(x-x_{2}\right)\right]\right\} \quad$ for $x_{1}<x<x_{3} \quad$ Eq. 98

$\begin{array}{lll}Y(x)=0 & \text { for } x>x_{3} & \text { Eq. } 99\end{array}$

Where $\mathrm{a}=4.14 \mathrm{~cm}$;

$h=0.5 h \max \left[1-\cos \left(2 \pi t^{*}\right)\right] \quad$ Eq. 100

$\mathrm{t}^{*}=\mathrm{t}-\mathrm{to} / \mathrm{T}, \mathrm{hmax}=0.38, \mathrm{~T}=5.33(\mathrm{~s}) \quad$ Eq. 101

The Reynolds number $(\mathrm{Re})$ based on the inlet bulk velocity $(U)$ and channel width (b) was $\operatorname{Re}=507$. The Struohal number (St) was $S t=b / U T=0.037$.

Here we demonstrated that the CFD engine from CFX ${ }^{\circledR}$ (Ansys, Canonsburg, PA) can calculate the flow physics shown in the visualization experiments (Pedley and Stephanoff, 1985). A fully structured mesh of the channel was created comprising of 20,000 elements as seen in Figure 16. The mesh after the deformation can be observed in Figure 17. In the simulations, the inlet velocity was $65.789 \mathrm{~mm} / \mathrm{sec}$, density was set to $1000 \mathrm{~kg} / \mathrm{m}^{3}$, viscosity was $1.079 \times 10^{-3}$ Pa.s, and the time step used was $0.01 \mathrm{sec}$. Using the equations that govern the plate displacement, we implemented a moving boundary simulation. They reported the results as a function of normalized time where 0 was the beginning of the cycle and 1 was the end of the cycle. One cycle was equal to 4 seconds. The time steps specified was $t^{*}=0.01$. The simulation was run during four cycles to allow for periodicity. 


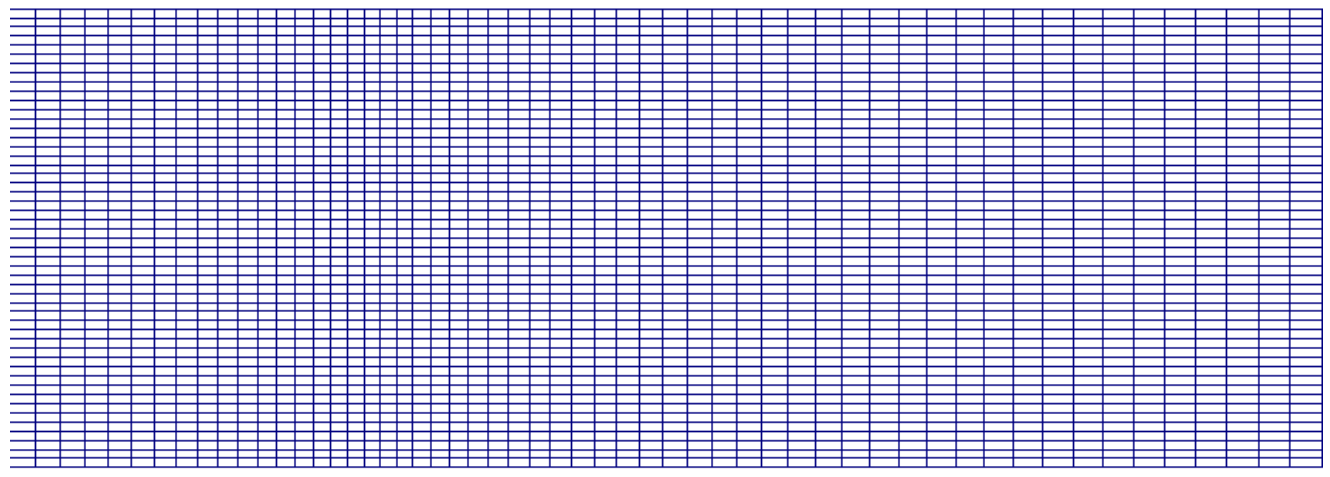

Figure 16. CFD Mesh - 20,000 Hexagonal Elements. 0 seconds (undeformed).

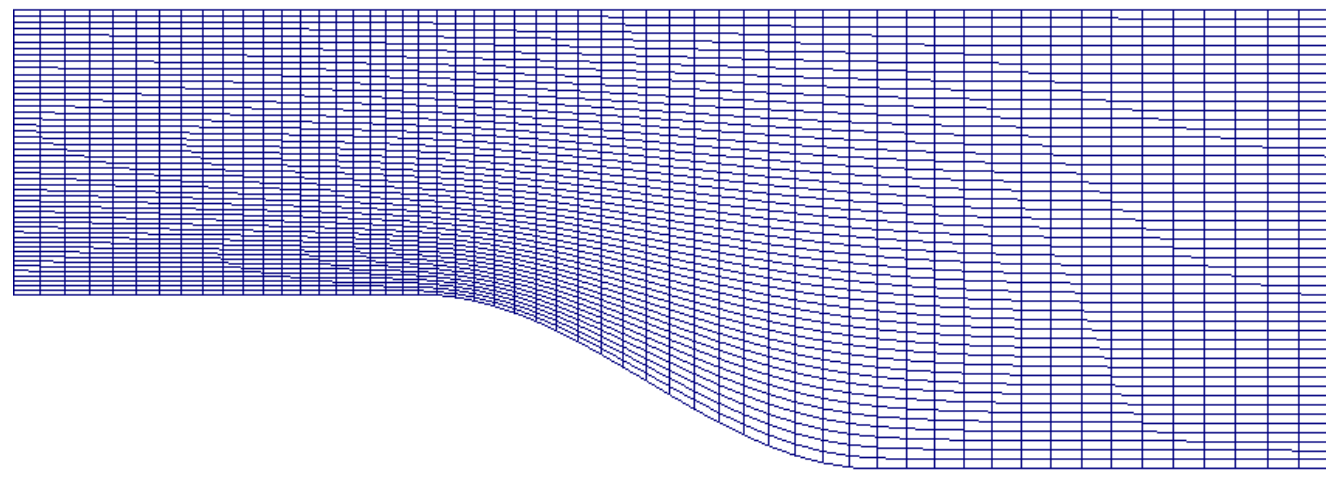

Figure 17. CFD Mesh - 20,000 Hexagonal Elements.

Pedley and Stepanoff (Pedley and Stephanoff, 1985) observed that the flow was unidirectional for the first 0.34 normalized time. At 0 the experiment showed a single recirculation region. Existence of multiple eddies where reported in following times as shown in Figure 18. CFD velocity vector plots are shown in Figure 19 where it can be seen that $\mathrm{CFX}^{\circledR}$ (Ansys, Canonsburg, PA) was able to precisely replicate the flow vortices observed during the flows visualization 
experiments performed by Pedley and Stephanoff (Pedley and Stephanoff, 1985).

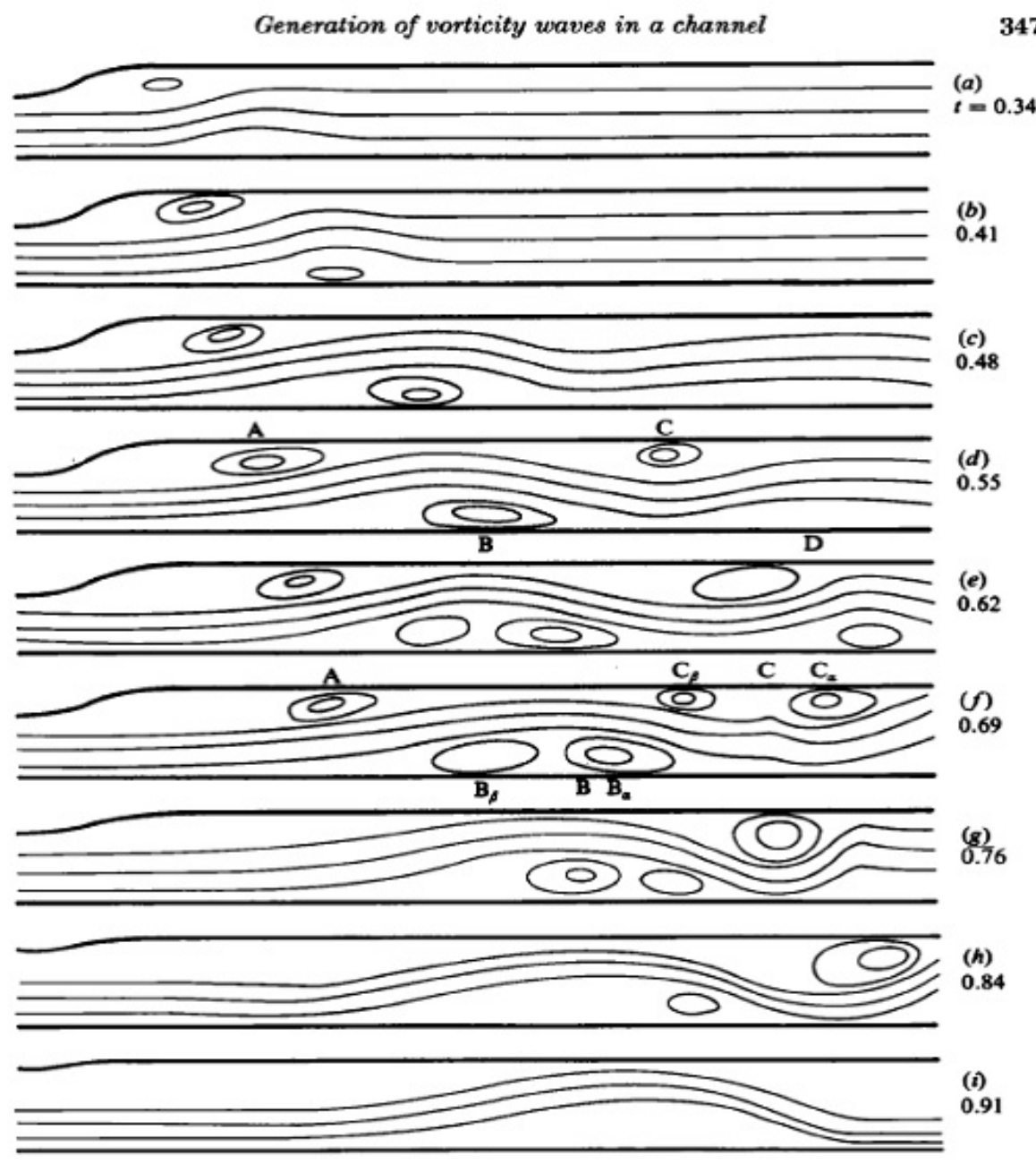

Figure 18. Simplified sketch of the flow patterns observed by (Pedley and Stephanoff, 1985). Source: Pedley T.J. and Stephanoff K.D., (1985), Flow along a Channel with a Time-Dependent Indentation in One Wall: the Generation of Vorticitv Waves. Journalof Fluid Mechanics, Volume 160, pp.337-367. 


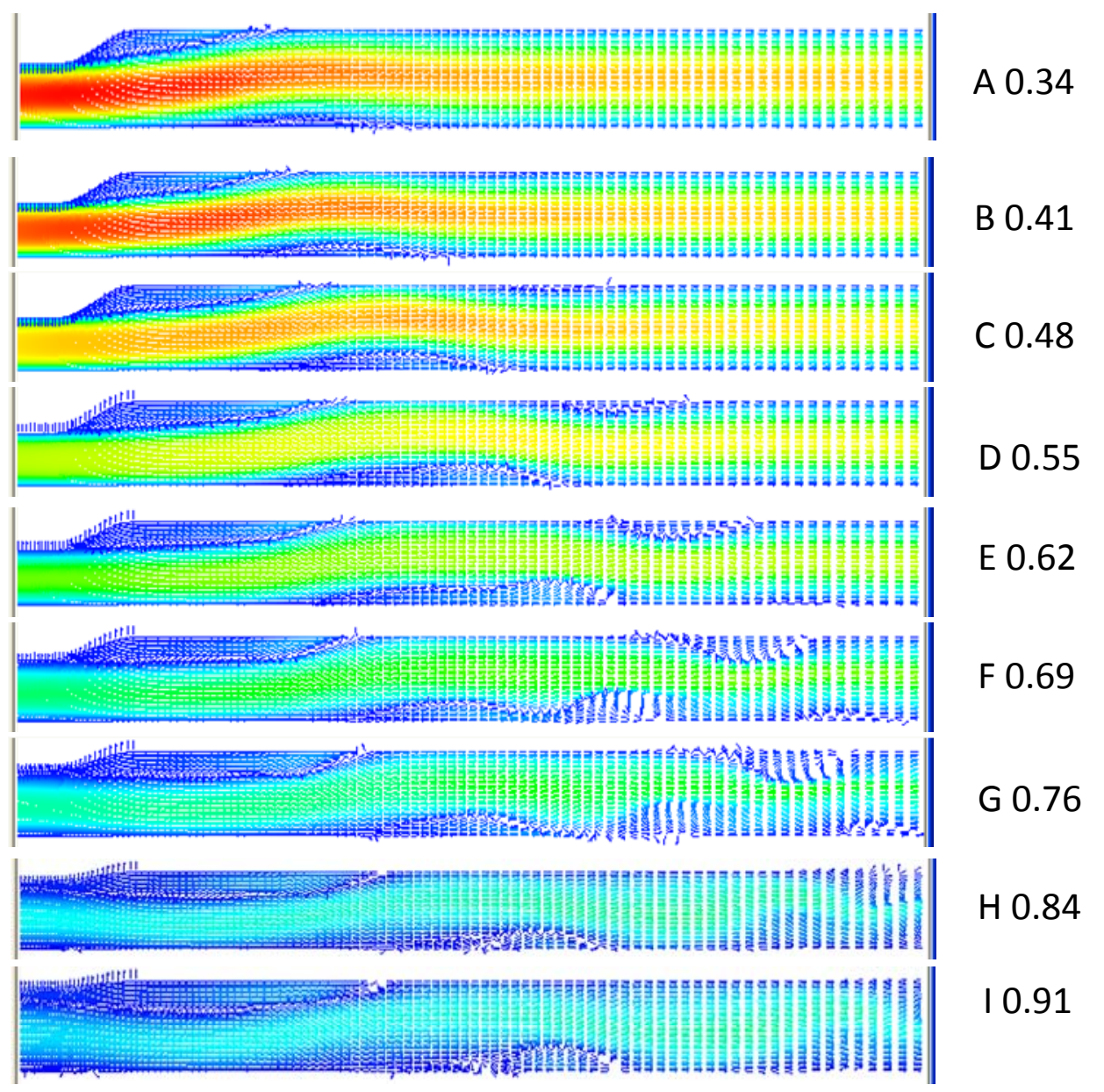

Figure 19. CFD replication of flow experiments performed by (Pedley and Stephanoff, 1985).

\subsubsection{CFD validation of specimen dynamic simulations}

We compared our shear stress results (time step $=2 \mathrm{~ms}$ ) for our flex-flow simulations with a similar dynamic simulation (time step $=0.5 \mathrm{~ms}$ ) conducted by Ramaswamy et al. (Ramaswamy et al., 2014) which employed a custom-written code based on the immersed boundary method and utilized a sample with dimensions of $1 \mathrm{~mm} \times 7 \mathrm{~mm} \times 25 \mathrm{~mm}$. In this study, our sample utilized dimensions of $1 \mathrm{~mm} \times 6.5 \mathrm{~mm} \times 17 \mathrm{~mm}$. Our results for shear stress were found 
to be within $10 \%$ of the previously reported shear stress and thus in good agreement.

The justification of a $2 \mathrm{~ms}$ time step in the current study can be further explained as follows: previously, we conducted a time independence study (Salinas et al., 2014a; Salinas et al., 2014b) of CFD simulations involving the FSF bioreactor using time steps of $0.5 \mathrm{msec}, 1 \mathrm{msec}, 2 \mathrm{msec}$, and $4 \mathrm{msec}$. The results showed no significant differences in the shear stress profiles ( $\sim$ less than $2 \%$ variability). In light of this fact, we applied a 2 msec time step for capturing the flow physics of the FSF, u-shaped bioreactor. We observe that in addition, there are several works that have previously reported CFD results obtained from simulations run using FSF-type bioreactor geometries, implementing a 2 msec time step (Salinas and Ramaswamy, 2014; Salinas et al., 2014b; Salinas et al., 2014a; Ramaswamy et al., 2014; Boronyak et al., 2009; Ramaswamy et al., 2008; Engelmayr et al., 2008).

We further note that a useful parameter that can aid in determining the most appropriate time step is the the courant number (CFL). It is given by:

$$
C=\Delta t \sum_{i=1}^{n} \frac{u_{x_{i}}}{\Delta x_{i}} \leq C_{\max }
$$

Where the dimensionless number is called the Courant number, $u$ is the magnitude of the velocity (length/time); $\Delta t$ is the time step (time); $\Delta x$ is the length interval (length). A CFL number that has a value of less than one is important in order to achieve convergence of the finite difference approximation. The courant number (CFL) in all of our simulations was below 0.95 and thus in 
compliance with acceptable convergence of the governing equations in the CFD studies we performed.

Going back to the study that utilized the IBM to resolve the flow field in the FSF, u-shaped bioreactor (Ramaswamy et al., 2014) we strongly believe that the $10 \%$ difference in shear stresses that was found compared to our corresponding simulation was mainly due to the differences in sample dimensions and the time steps between the two investigations. Nonetheless, we state that the simulations

performed by (Ramaswamy et al., 2014) were highly precise while comparatively, the ones conducted in this study were still within a reasonable degree of accuracy (10\%) and could thus be used to evaluate relative flow physics among the different bioreactor CFD simulations conducted (e.g. combined cyclic specimen flexure and steady flow versus cyclic flexure-alone).

\subsection{Results}

The fluid-induced, time averaged axial shear stresses were plotted on the inner and outer surfaces for all the simulation cases (Figure 20). The values were found to be higher on the outer wall for all cases except the control (no flow, no flexure) wherein they were essentially the same on both walls. The highest shear stress values were observed to occur at the vertex of the samples. This finding is consistent with the previous report by (Engelmayr et al., 2008). In addition, the prediction of amplified wall shear stresses at the vertex of the samples was indicated in (Engelmayr et al., 2006) as a potential mediator of tissue formation. 
Also, the flex-flow simulations showed augmented shear stresses relative to steady flow-alone or specimen cyclic flexure-alone.

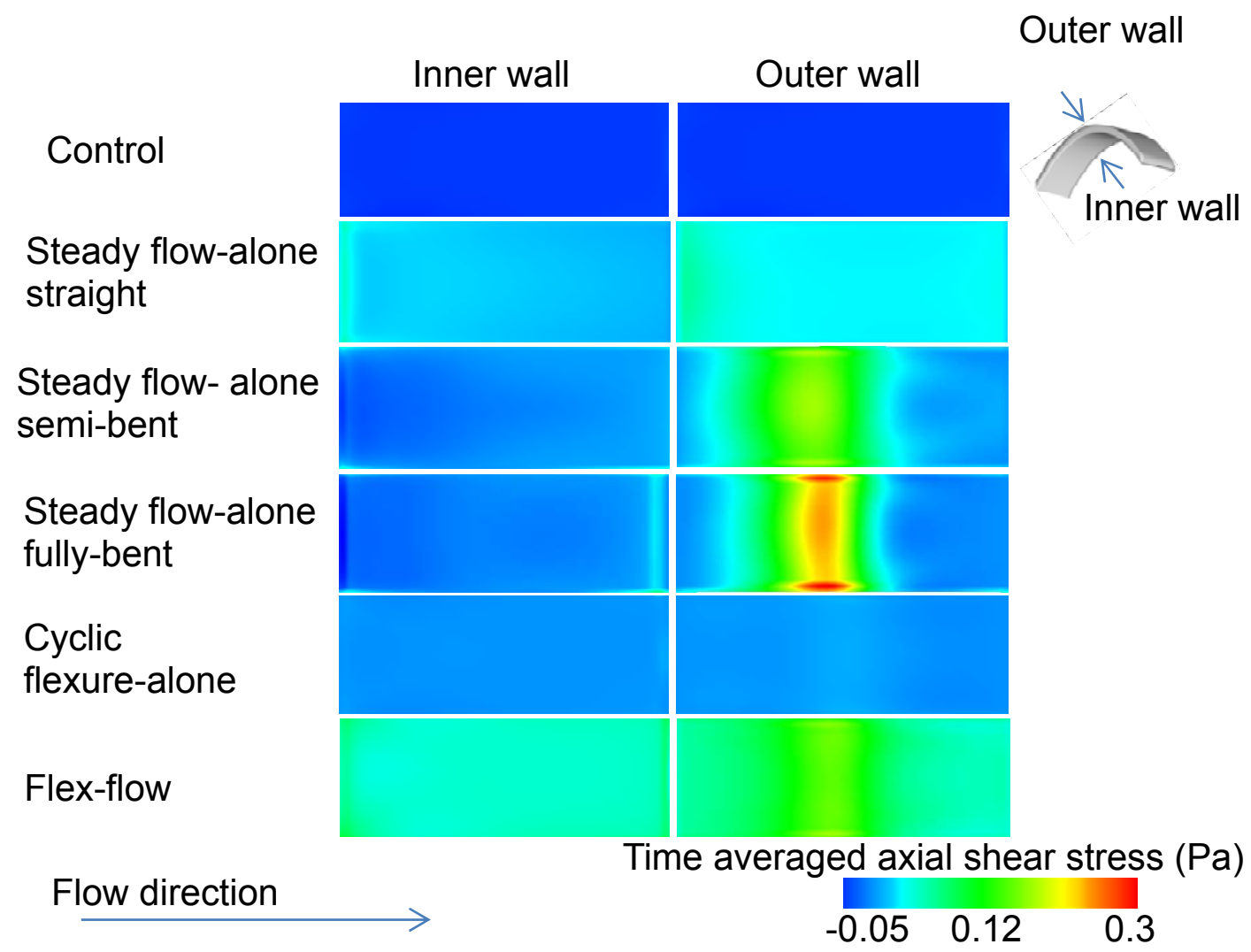

Figure 20: Fluid-induced, time-averaged axial shear stresses over one cycle on specimen inner and outer walls for the following six cases: i) no flow and no flexure ii) steady flow-alone (straight sample), iii) steady flow-alone (semi-bent sample), iv) steady flow-alone (fully-bent sample), v) cyclic flexure-alone and vi) flex-flow states. Shear stresses were found to be higher along the outer wall except in the case of no flow and no flexure. The highest shear stresses were observed around the vertex of the sample. In comparing the two dynamic cases (v) versus (vi), the flex-flow state displayed much higher shear stress values.

To observe the velocity profiles at a section surrounding the vicinity of the samples, a longitudinal cross-sectional plane $(x=0.0175 \mathrm{~m})$ at the center line of 
the specimens was applied (Figure 21). The results showed non-existent to modest levels of flow separation in the steady flow-alone cases; when the samples were straight the flow was fully unidirectional in the forward flow direction (Figure 21a). On the other hand, a few vortices proximal to the inner specimen wall were observed in the semi-bent and fully bent configurations.

Substantially higher flow reversal regions were observed in the case involving cyclic flexure-alone (Figure 21b). Complete recirculation zones occurred simultaneously with the initiation of sample bending $(t=0 \mathrm{~s})$. Loss of uniformity in the size and pattern of vortices in the flow field increased at subsequent time steps. In addition, the velocity streamlines were different during the bending $(t=0.25 \mathrm{~s})$ and unbending $(t=0.75 \mathrm{~s})$ events, even though the sample configurations at these time instances were identical. Meanwhile, in the flex-flow case (Figure 21c), the number of flow vortices increased with the specimen flexure states but was not as pronounced as those observed in comparison to cyclic flexure-alone (Figure 21b). On the other hand, in the flexflow environment, there was greater variability in the axial velocity distribution. 


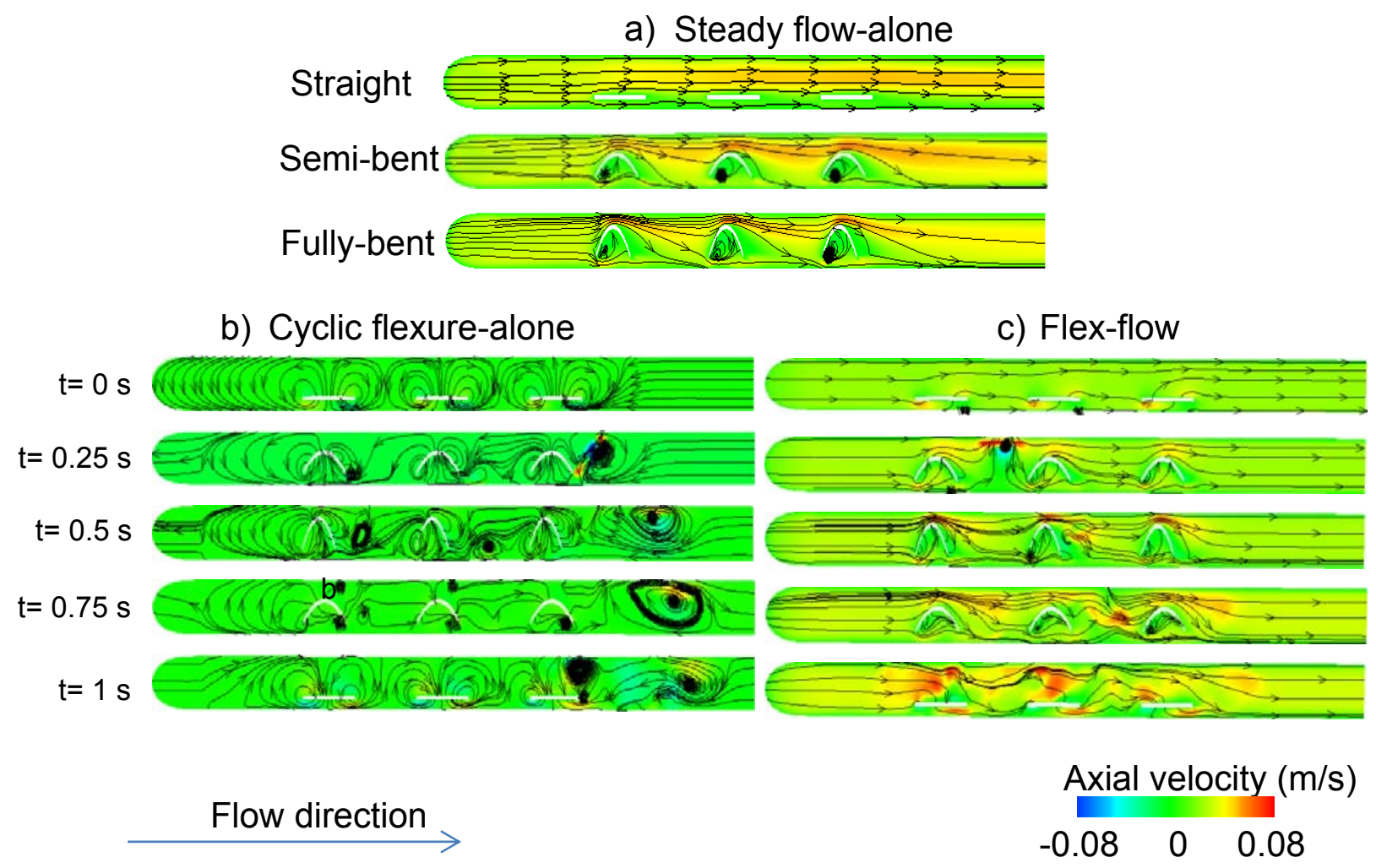

Figure 21: Contour of velocity magnitudes and velocity streamlines in the plane $(x=17.5 \mathrm{~mm})$ surrounding the specimens for the following cases: a) steady flowalone (straight sample), b) cyclic flexure-alone and c) flex-flow states. The inlet velocity for a) and c) was set to $0.022 \mathrm{~m} / \mathrm{s}$ while for $b$ ) it was set to $0 \mathrm{~m} / \mathrm{s}$. A large degree of vortices formed in cyclic flexure-alone case, but the magnitude of velocity field was clearly lower when compared to steady flow-alone and flex-flow cases. It was also noted that the flow physics during the specimen flexing and straightening events (in both $b$ ) and c) at the instance when the specimen shape was identical, i.e., at $t=0.25$ and $0.75 \mathrm{~s}$ respectively were not the same.

In order to quantify the extent of directionality changes in the temporal shear stress experienced by the samples, i.e., OSS under cyclic flexure-alone and flex-flow conditioning, the oscillatory shear index (OSI) was calculated. As previously defined by $\mathrm{He}$ and $\mathrm{Ku}(\mathrm{He}$ and $\mathrm{Ku}, 1996)$ the equation for the OSI is given by: 
OSI $=\frac{1}{2}\left(1-\frac{\operatorname{abs}\left(\int_{0}^{T} \tau d t\right)}{\int_{0}^{T} a b s(\tau) d t} \quad\right.$ Eq. 102

Where, $\operatorname{abs}\left(\int_{0}^{\mathrm{T}} \tau \mathrm{dt}\right)$ is the magnitude of the time-averaged shear stress while $\int_{0}^{\mathrm{T}} \operatorname{abs}(\tau) \mathrm{dt}$ is the time-averaged shear stress magnitude. One can observe that: $0.0 \leq \mathrm{OSI} \leq 0.5$, where an $\mathrm{OSI}=0.0$ represents unidirectional flow while OSI $=$ 0.5 signifies complete oscillatory flow.

Consistent with the axial velocity plots (Figure 21), the OSI distribution was found to be much higher when exposed to cyclic flexure-alone, relative to flex-flow conditions (Figure 22a). The area averaged OSI on the specimen inner walls $(n=3)$ was 0.433 and 0.230 for cyclic flexure-alone and flex-flow cases respectively while the corresponding values for the outer wall $(n=3)$ was found to be 0.313 and 0.076 . For the dynamic simulations, as a means to represent the coupled effects of shear stress magnitude and OSS, we defined the product of a specimen's OSI distribution and its corresponding time averaged shear stress magnitude (TSSM), multiplied by a factor of two, as the "OSI-scaled shear stress magnitude" (OSI- $\mid \overrightarrow{|\tau|})$, i.e.,:

\section{OSI- $\overrightarrow{|\tau|}=2$ *OSI * TSSM $\quad$ Eq. 103}

The OSI- $\overrightarrow{|\tau|}$ distribution was in general, relatively higher on the specimen surfaces (Figure 22b) under flex-flow states compared to cyclic flexure-alone.

The area averaged OSI- $\overrightarrow{|\tau|}$ for the inner walls were $0.01 \mathrm{~Pa}$ (cyclic flexure-alone) and 0.066 Pa (flex-flow) while for the outer walls these were 0.016 Pa (cyclic flexure-alone) and 0.028 Pa (flex-flow). 


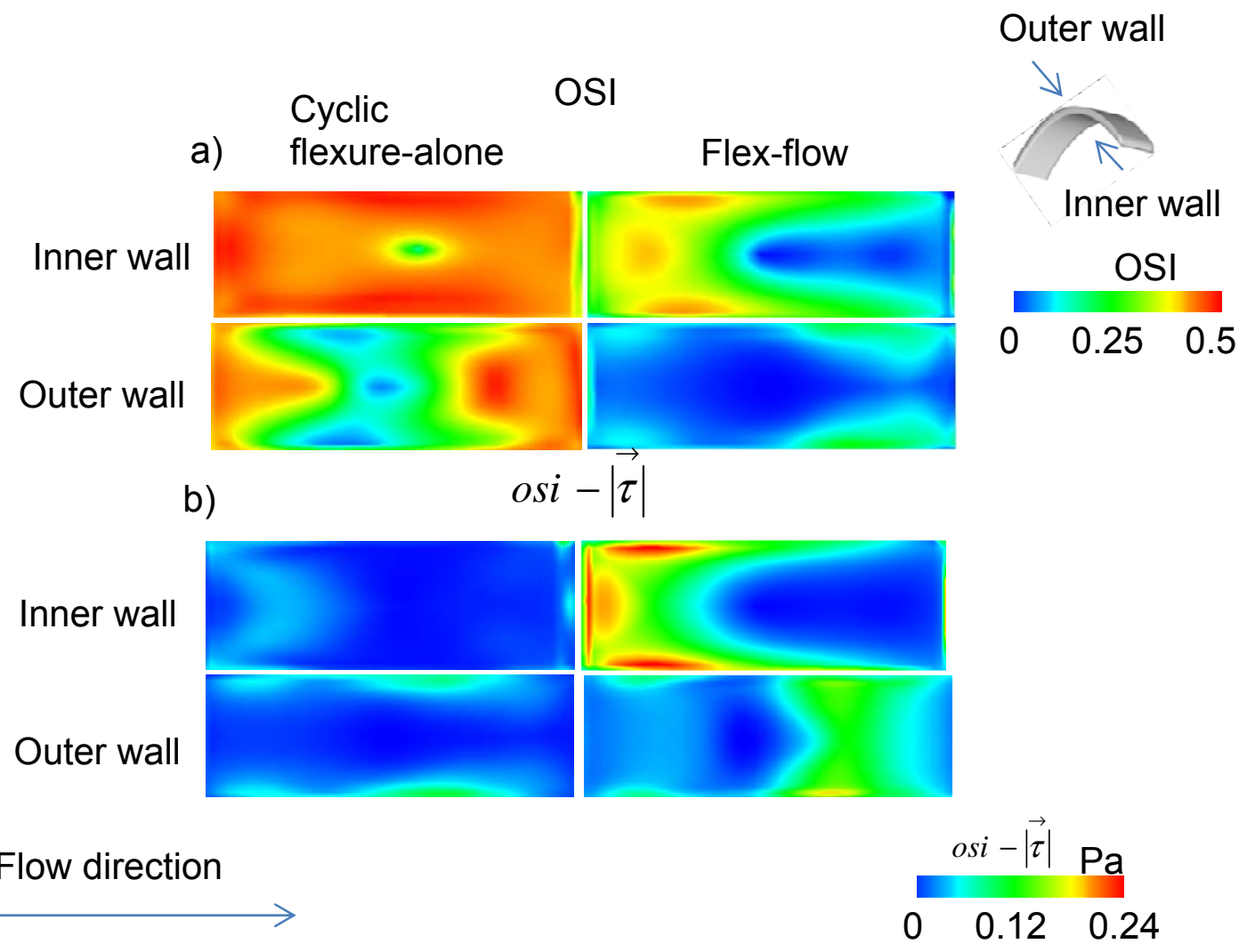

Figure 22: a) OSI on the inner and outer specimen surface for the dynamic cyclic flexure-alone and flex-flow cases. The node averaged OSI along the inner walls were 0.433 (cyclic flexure-alone) and 0.230 (flex-flow) while for the outer walls the OSI was 0.313 (cyclic flexure-alone) and 0.076 (flex-flow). b) OSI-scaled shear stress magnitude (osi-| $\overrightarrow{\boldsymbol{\tau} \mid})$ on the inner and outer specimen surface for the dynamic cyclic flexure-alone and flex-flow cases. The node averaged osi-| $|\overrightarrow{\boldsymbol{\tau}}|$ along the inner walls were $0.01 \mathrm{~Pa}$ (cyclic flexure-alone) and $0.066 \mathrm{~Pa}$ (flex-flow) while for the outer walls the osi-| $|\overrightarrow{\boldsymbol{\tau}}|$ was $0.016 \mathrm{~Pa}$ (cyclic flexure-alone) and 0.028 $\mathrm{Pa}$ (flex-flow). Effectively the osi- $|\overrightarrow{\boldsymbol{\tau}}|$ metric served to combine both the time averaged and oscillatory shear stress components, demonstrating that its magnitude was much lower in cyclic flexure-alone states compared to flex-flow states, even though the OSI of the former was substantially higher compared to the latter.

Previously, Engelmayr et al. (Engelmayr et al., 2006) defined a percent increase in collagen content relative to the average collagen content of the no 
flow, no flexure (control group), steady flow-alone, cyclic flexure-alone and flexflow groups. When plotting the TSSM, OSI and OSI-|ح| metrics as a function of these previously reported collagen content amounts, only OSI- $\overrightarrow{|\tau|}$ was found to be in direct proportion to the substantially augmented engineered collagen that had formed when cyclic flexure and steady flow conditions were combined (Figure 23). In a similar fashion, we quantified the net $\%$ increase in the mean TSSM, OSI and OSI-| $\overrightarrow{\tau \mid}$ parameters that resulted from flex-flow mechanical conditioning in comparison to the sum of the corresponding parameter amounts resulting from cyclic flexure-alone and steady flow-alone conditioning, as follows:

$\%$ increase $=$

$\frac{\left(\text { Parameter }_{\text {Flex-Flow }}\right)-\left(\text { Parameter }_{\text {Cyclic Flexure-alone }}+\text { Parameter }_{\text {Steady Flow-alone })}\right.}{\left(\text { Parameter }_{\text {Cyclic Flexure-alone }}+\text { Parameter }_{\text {Steady Flow-alone }}\right)} \times 100 \quad$ Eq. 104

where "Parameter" Refers to TSSM, OSI or OSI-||. The \% increases for TSSM, OSI and OSI-|ح| were found to be, $27.65 \%,-37.19 \%$, and $207.14 \%$ respectively. 
a)

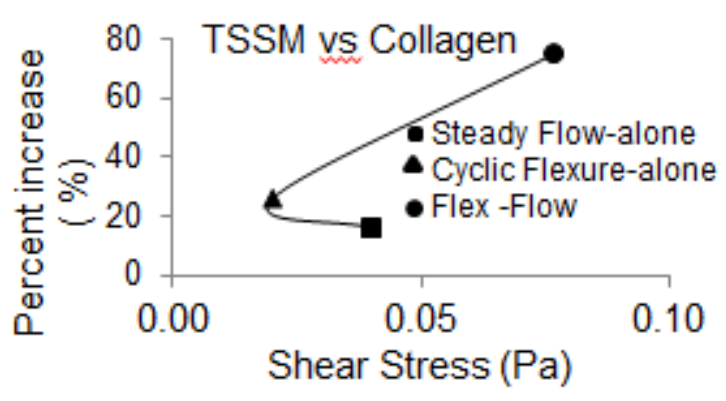

b)

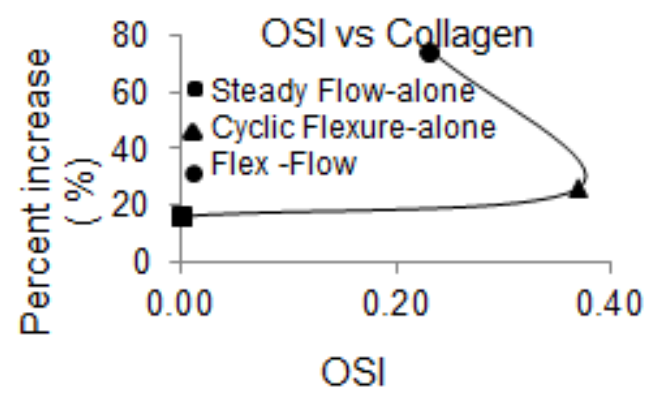

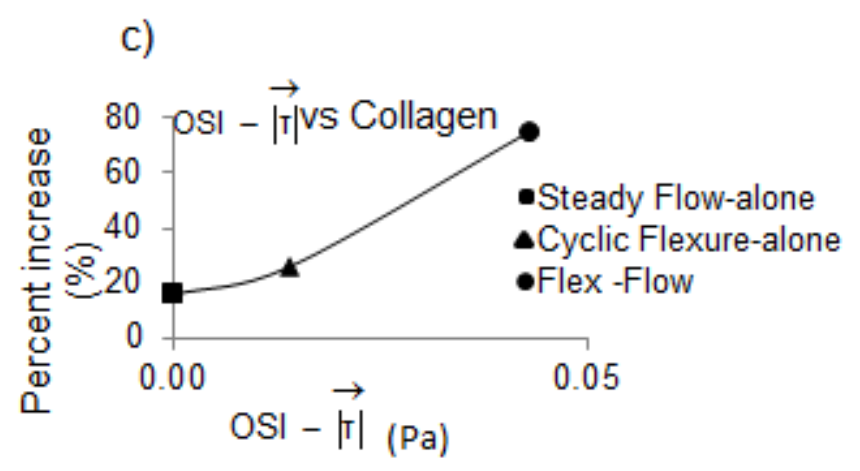

Figure 23: For the following three cases of 1) steady flow-alone (straight sample), 2) cyclic flexure-alone and 3) flex-flow states, the specimen area mean: a) TSSM, b) OSI and c) OSI-| $\overrightarrow{\boldsymbol{\tau}} \mid$ was plotted vs. the percentage (\%) collagen increase. In a) b) and c), the \% increase in collagen was observed relative to the average of the sum of no flexure and no flow, steady flow-alone (straight sample) and cyclic flexure-alone, as previously reported by (Engelmayr et al., 2006) Only the OSI-| $\overrightarrow{\boldsymbol{\tau}} \mid$ parameter demonstrated a convincing correlation to the collagen production found in flex-flow states (Engelmayr et al., 2006), which resulted in significantly higher engineered tissue collagen content.

\subsection{Discussion}

Shear stress is known to be an important promoter of engineered tissue formation (Engelmayr et al., 2008; Engelmayr et al., 2006; Karim et al., 2006). In addition to the magnitude of these stresses, studies have independently suggested that temporal changes in the directionality of shear stress, i.e., OSS is also critical for promoting tissue formation and phenotype (Li et al., 2004; 
Ramaswamy et al., 2010). For example, the importance of OSS has directly been associated with the regulation of the KLF2-a gene which is essential for normal valvulogenesis (Vermot et al., 2009).

In this study we sought to computationally simulate the localized flow physics surrounding engineered tissue specimens that could have ultimately been responsible for the significant increases in collagen tissue production reported in previous heart valve tissue engineering investigations, under the combination of steady flow and sample cyclic flexure (Engelmayr et al., 2006). Thus, in this investigation, we simulated, no flow-no flexure (control), steady flow-alone, cyclic flexure-alone and flex-flow conditions of samples that underwent mechanical conditioning in a bioreactor; to incorporate the bending motion of the samples, the cyclic flexure-alone and flex-flow simulations both required moving boundary computational simulations which we implemented using the ALE formulation.

Previously Engelmayr et al. (Engelmayr et al., 2008) predicted that the peak wall shear stress magnitudes on rectangular-shaped specimens occurred at the upstream, convex edge of the flexed samples and approached $1 \mathrm{~Pa}$, while the corresponding average value was $0.182 \mathrm{~Pa}$. In this study, the peak wall shear stress magnitude was found to be $0.30 \mathrm{~Pa}$, occurring at the same spatial location on the specimens (specimen vertex along the outer wall, steady flow-alone, fullybent case; Figure 20), while the average wall shear stress magnitude was 0.05 $\mathrm{Pa}$. As observed in both current and previous investigations (Engelmayr et al., 2008) on conditioned rectangular-shaped, flexed specimens, the peak specimen 
shear stress magnitudes were roughly five to six times higher than the average. However, the shear stress magnitudes in the current study were much lower than those reported by Engelmayr et al. (Engelmayr et al., 2008) primarily because of the relatively lower inlet velocity boundary condition that was prescribed $(0.022$ $\mathrm{m} / \mathrm{s}$ here versus 0.08 by (Engelmayr et al., 2008). Our inlet velocity was based on the time-averaged flow conditions of the human pulmonary artery (Lotz et al., 2002).

The major novelty of our study is that we identified a new biomechanical parameter in the form of a scaled OSI (OSI-|ح|) that correlates with the previously reported observation (Engelmayr et al., 2006) that, greatly augmented engineered collagen formation occurred solely when steady flow and cyclic flexure were combined, i.e. the flex-flow state. On the other hand, neither TSSM nor OSI alone could convincingly explain the large increases in collagen content derived from this flex-flow activity.

Clearly OSI-|च| yielded a much higher value, roughly 7.5 times larger than the percent increase in TSSM alone (Figure 23a). On the other hand, any correlation with OSS proved to be incorrect since the combined flex-flow states suggested less engineered collagen content relative to the cyclic flexure-alone case (Figure 23b). We believe that the reason for this was because, while specimen cyclic flexure-alone is highly effective at generating OSS (Fig 17a), it is obviously not accompanied by appreciable shear stress magnitudes, which are typically facilitated by a flow source, such as a pump. Specifically, the case of cyclic flexure-alone comprises of relatively low magnitudes of fluid shear stress 
arising only from the motion of the cyclically flexing specimens in an effectively static fluid bath. Clearly, this is an example of a mechanical conditioning environment of relevance to heart valve tissue engineering where the OSS is high but the shear stresses are low, which previously led to insignificant levels of engineered tissue content, relative to no flow, no flexure static controls (Engelmayr et al., 2006). This observation demonstrates yet again that the coexistence of both metrics, i.e., OSS and TSSM are important for engineered collagen formation.

The \% increase reported by Engelmayr et al. (Engelmayr et al., 2006) in engineered collagen production under flex-flow states compared to the average collagen content of the no flow, no flexure, steady flow-alone and cyclic flexurealone conditions was $75 \%$ whereas the corresponding increase for the sum of independent cyclic flexure-alone and steady flow-alone was only $42 \%$. Similarly, the OSI- $\overrightarrow{\tau \mid}$ quantity under flex-flow states was $0.044 \mathrm{~Pa}$ while the corresponding value for the sum of independent cyclic flexure-alone and steady flow-alone was 0.014 Pa. In other words, significant increases in engineered collagen content under flex-flow states correlated with an appreciable, corresponding increase in the OSI-|ح| parameter (Figure 23c).

The goal of the OSI-|ح| parameter is to emphasize that BOTH the timeaveraged shear stress magnitude (TSSM) and OSI are important in promoting engineered heart valve tissue formation as was previously observed in the experiments of Engelmayr et al. (Engelmayr et al., 2006). As a result we define 
OSI- $\overrightarrow{|\tau|}$ as a single index that can capture the necessity for both OSI and TSSM metrics to be present during heart valve tissue engineering experiments. A factor of two is multiplied in the definition of OSI-|च| for the following reason: When the OSI is maximum, i.e., OSI $=0.5$, the OSI-| $\overrightarrow{\tau \mid}$ simply reduces to TSSM. On the other hand, as one would observe during steady flow-alone or cyclic flexurealone mechanical conditions, OSI-| $\overrightarrow{|\tau|}$ would be zero and negligible respectively. We note again that the findings of Engelmayr et al. (Engelmayr et al., 2006) suggested that neither steady flow-alone or cyclic flexure-alone showed any significant augmentation in engineered collagen formation compared to static tissue culture (no flow-no flexure, control group). Therefore indeed, in these cases, we would expect that the biomechanical conditioning "effect" of OSI-| $\overrightarrow{\tau \mid}$ to be zero or negligible. Thus, we have in a simple way shown that neither TSSM nor OSI alone can convincingly explain significant increases in collagen production that Engelmayr et al. (Engelmayr et al., 2006) reported under a combination of flex-flow states. On the other hand, the combination of these two parameters, characterized by OSI-|T| does capture the synergy of the flex-flow state (in the context of promoting engineered collagen production activity). In the flex-flow case the average specimen OSI was not a maximum of 0.5 but was instead found to be $\sim 0.23$. Thus, the OSI-|ح| parameter served to reduce the mechanical conditioning "effect" of TSSM, by a factor of 0.46 (i.e., $2{ }^{*} \mathrm{OSI}$ ), in the flex-flow case. 
We note however that OSI-| $\overrightarrow{\tau \mid}$ needs to be interpreted in a relative rather than an absolute context, for example in comparing flex-flow versus steady flowalone or versus cyclic flexure-alone. Certainly the magnitude of TSSM and OSI can have independent effects on the amount of engineered tissue formation. However, this amount when obtained from a steady flow-alone (TSSM-only) or cyclic flexure-alone (OSI-only) experiment will always be small relative to a corresponding experiment conducted under flex-flow states (both TSSM and OSI) which utilizes the same magnitude of TSSM and OSI as when they were used independently. In addition, in this first approximation study, the OSI-|ح| metric also assumed that both OSI and TSSM are equally important, although this remains to be proven. Finally, since the Engelmayr et al. (Engelmayr et al., 2006) study was based on bone marrow stem cells (BMSCs) the interpretation of OSI$\overrightarrow{|\tau|}$ can for the time-being only be used for this cell type since the response of other cell types to mechanical stimuli and subsequent rates of engineered collagen synthesis may be different. We believe nonetheless that this distinction, i.e., OSI- $\overrightarrow{|\tau|}$ is important (as opposed to simply TSSM alone or OSS alone). This is because OSS can be generated without a flow source and purely through specimen movement, while non-moving specimens exposed to a steady flow source that generate physiological-levels of shear stresses are not exposed to oscillatory effects; yet the simultaneous presence of shear stress magnitude and temporal changes to the directionality in flow seem to be required to promote engineered heart valve tissue formation. 
In conclusion, we present a modification of the traditional OSI parameter first defined by $\mathrm{He}$ and $\mathrm{Ku}(\mathrm{He}$ and $\mathrm{Ku}, 1996)$, by multiplying it to its spatially corresponding time-averaged, fluid-induced shear stress quantity (TSSM) and by a factor of two. In doing so, we were able to demonstrate that the combination of both parameters $(\mathrm{OSI}-\overrightarrow{|\tau|})$ correlated strongly with the greatly augmented engineered collagen production reported from previous in vitro experiments (Engelmayr et al., 2006), under flex-flow conditioning regimens. We wish to emphasize however, that a limitation of our study was that the OSI-| $\overrightarrow{\tau \mid}$ metric we defined assumed that both OSI and TSSM are equally important, but this may or may not be the case and remains to be determined. Our principal goal behind OSI- $\overrightarrow{|\tau|}$ was to demonstrate that $\underline{\text { both }}$ the magnitude and directionality (i.e., oscillations) of the fluid-induced shear stresses are critical in augmenting tissue formation, rather than to arrive at a precise metric that can be used to quantify the relative contributions of OSI and TSSM. Indeed as stated earlier, our definition of OSI- $\overrightarrow{|\tau|}$ is by no means an ideal metric and remains at best, a crude approximation. Nonetheless, in this particular study OSI-| $\overrightarrow{\tau \mid}$ did correlate with increased collagen formation. However given that there is a dependence on the biological response of different cell types to mechanical environments, the correlation could be altered if cells other than BMSCs are used in the tissue engineering experiments. 


\section{CHAPTER 5: MASS TRANSPORT MODEL}

The most severe forms of congenital heart disease have an incidence rate of $\sim 20,000$ live births/year (Hoffman, 1995). A large number of these cases present with problems associated with the heart valves. Moreover, an estimated 104,000 valve procedures are performed in the US (Roger et al., 2011) alone annually and this number continues to grow each year. Today, replacement of a defective valve with a prosthetic heart valve is commonplace in adult and pediatric patients. However, despite recent advances in medical technology, distinct limitations remain in the current treatment of critical, congenital heart valve disease. Even though prosthetic valves are currently used to replace defective valves, no currently available treatment option provides a permanent solution. Among the pediatric population, the situation is further aggravated by the inability of the valve substitute to permit somatic growth thus necessitating the child to undergo multiple invasive operations. From a research standpoint, functional, organ-level, tissue engineered heart valve (TEHV) replacement which would permit somatic growth and would not pose any immune risks was thought to represent the Holy Grail in treating valve disease. For example, extensive work in the heart valve tissue engineering area has been conducted by the Mayer and Hoerstrup groups (Hoerstrup et al., 2000a; Hoerstrup et al., 2000b; Hoerstrup et al., 1998; Hoerstrup et al., 1999; Sodian et al., 2000a; Sodian et al., 1999;

Sodian et al., 2000b; Sodian et al., 2000c). They showed that when implanted in the pulmonary artery position, TEHVs functioned (observed for four months) in growing lambs, exhibiting durability, growth, and lack of thrombogenicity 
(Hoerstrup et al., 2000a; Sutherland et al., 2005). Yet, after more than a decade of intensive TEHV investigations which included clinical studies (Dohmen et al., 2011), identification of a specific protocol for optimized construct development which can subsequently be clinically translated remains elusive. While potential cell sources and scaffold materials for heart valve tissue engineering have been identified (Chen and Wu, 2005; Fong et al., 2006; Frank et al., ; Hilfiker et al., 2011; Kadner et al., 2002; Leor et al., 2005; Lichtenberg et al., 2006; Martinez et al., 2013; Neuenschwander and P. Hoerstrup, 2004; Perry et al., 2003; Schmidt et al., 2006; Schmidt et al., 2010; Siepe et al., 2008; Simon et al., 2006; Weber et al., 2011a; Weber et al., 2011b), the biomechanical conditions needed to promote the heart valve phenotype still require further investigation. In addition, cellular responses to the applied mechanical stresses will undoubtedly depend on the cell type(s) being utilized; adult progenitor cells, such as bone marrow derived stem cells (BMSCs) are deemed likely candidates for utility in heart valve tissue engineering owing to their pluripotency, ease of access and lack of ethical concerns. Given that valvulogenesis is closely tied to its surrounding hemodynamics, it has been generally accepted that this biomechanical environment to the extent possible, be recapitulated during engineered heart valve tissue culture. However, the specifics such as the manner in which mechanical stresses need to be applied, the necessity to deform the growing construct similar to a native valve and the duration of the culture process remain uncertain. 
To further understand in vivo native heart valve stress environments, several efforts have focused on the innate stresses that native heart valves are subjected to, namely, fluid-induced shear stresses and the cyclic tissue stretch and flexure events that occur during valve opening and closure. Specifically, custom-made heart valve bioreactors have been used to study the individual or combined effects of these stresses on engineered tissue formation (Engelmayr et al., 2003; Engelmayr et al., 2008; Ramaswamy et al., 2014; Ruel and Lachance, 2009), while computer simulations have focused on the quantification of stress states on valvular tissues (Engelmayr et al., 2008; Ramaswamy et al., 2014; Salinas et al., 2014a; Salinas et al., 2014b; Salinas and Ramaswamy, 2014; Stella et al., 2007). Some of the key mechanobiological findings were as follows: Engelmayr et al (Engelmayr et al., 2006) showed that BMSCs cultured under conditions of flow and scaffold flexure produced more collagen than conditions of flow or cyclic flexure-alone. Vermot et al. (Vermot et al., 2009) reported that oscillatory shear stresses (OSS) directly regulated the gene klf2a which is required for normal valve development. We previously showed (Salinas and Ramaswamy, 2014) that robust collagen production from the combination of cyclic flexure and steady flow states (flex-flow) (Engelmayr et al., 2006) was a direct result of an appreciable shear stress magnitude tied to a high degree of flow oscillations, i.e., OSS. Moreover we also demonstrated that these types of OSS environments can be recreated under pulsatile flow conditions wherein the samples are fixed, i.e., do not move (Salinas et al., 2014a; Salinas et al., 2014b). 
The specific biomechanical environments used in the culture of engineered heart valve tissues will contribute towards the transport of nutrients and removal of metabolic waste products from the growing constructs (Brown et al., 2007; Chen et al., 2014; Ramaswamy et al., 2014; Salinas and Ramaswamy, 2014; Syedain, 2009b). Such transport is critical to the survival of cells, their phenotypic stability and subsequently the robustness of extracellular matrix that is produced. Indeed it has been shown that without adequate, homogeneous delivery of nutrients within cultured tissues, cell and matrix content will be adversely affected (Wang et al., 2008; Wang et al., 2011), with an overall decrease in the rate of its production.

Although mass transport in tissue culture involves multiple biochemical species, studies focused on other tissue types, such as skin, cartilage, oculartissues, and blood vessels have clearly established the importance of adequate oxygen (Abraham et al., 2013; Ai and Vafai, 2006; Chung and Vafai, ; Chung and Vafai, 2013; de Monte et al., 2013; Denny and Walsh, ; Ethier and Moore, 1997; Gill et al., 2011; Johnson et al., 2009; Kaazempur-Mofrad et al., 2005; Karner and Perktold, 2000; Khakpour and Vafai, 2008; Kumar et al., 2004; Liu et al., 2011; Prosi et al., 2005; Stangeby and Ethier, 2002; Tarbell et al., 1988; Wang et al., 2009) and glucose delivery. (Boubriak et al., 2006; Converti et al., 1996; Day et al., 2013; Gao and Xu, 2011; Koutny, 2011a; Koutny, 2011b; Martineau, 2012; Mokhbi Soukane et al., 2007).

Biochemical species is supplied to living tissues viatwo mechanisms: the first is convective mass transport while the second is through diffusive processes 
(Stangeby and Ethier, 2002; Yang and Vafai, 2006). Convective nutrient delivery is associated with pressure-driven transmural flow while the diffusive process is initiated by species-specific concentration gradients (Ai and Vafai, 2006; Ethier and Moore, 1997). These gradients are caused by nutrient consumption and production of metabolic wastes by the cells embedded within the tissues (Chung and Vafai, ). Mass transport models thus far have focused primarily on studying the distribution of oxygen in heart valve tissues due to convection and diffusion (Brown et al., 2007; Chen et al., 2014; Liu et al., 2011; Wang et al., 2008; Wang et al., 2011). However, they were limited to simplified diffusion models or did not account for oxygen consumption rates by cells. Moreover heart valve tissue engineering protocols typically employ a mechanical conditioning protocol wherein a flow and/or specimen movement (e.g. cyclic flexure) component is present (Engelmayr et al., 2006; Ramaswamy et al., 2010) the convectivediffusive processes that occur in these settings have thus far not been identified, but may play a critical role in maintaining construct viability for subsequent implantation.

From a biomechanics standpoint, we recently identified (Salinas and Ramaswamy, 2014) that measurable quantities of fluid-induced stresses accompanied by an oscillatory component were strongly correlated to the significantly higher collagen content found in constructs that were subjected to the combination of steady flow and specimen cyclic flexure (flex-flow) (Engelmayr et al., 2006). However, since these samples were moving, the enhanced tissue production may not have been due to only biomechanical effects; rather, the 
evolution of de novo tissues in dynamic culture systems involving conditions such as flex-flow may also be modulated by the efficiency of the mass transport mechanism(s). Therefore in this study, we built on our previous computational fluid dynamics (CFD) models (Salinas and Ramaswamy, 2014) to include mass transport components, specifically in the delivery of glucose and oxygen to the growing 3-dimensional (3D) engineered tissues. We correlated these findings with literature in vitro experiments (Engelmayr et al., 2006).

\subsection{Methods}

\subsubsection{Geometry, Mesh, and Simulation Set-up}

We modeled the FSF bioreactor previously described in (Ramaswamy et al., 2014; Salinas and Ramaswamy, 2014). We created a high density mesh of the fluid domain of the bioreactor as previously reported in (Salinas and Ramaswamy, 2014) with additional $4.5 \times 10^{4}$ tetrahedral elements in the sample region as seen in Figure 24 and imported it into the CFD solver.(CFX version, Ansys Inc., Canonsburg, PA). We replicated the simulations from (Salinas and Ramaswamy, 2014) where we analyzed cases of flow-alone, sample cyclic flexure-alone, flow and flexure, and a control group. The inlet boundary condition was set to a velocity of $0.02 \mathrm{~m} / \mathrm{s}$ for the cases of flex-flow and flowalone, and it was set to a velocity of zero for cases of cyclic flexure-alone and control. No slip conditions were prescribed to the bioreactor walls while a porous fluid-interface was set to the surface of the samples. All simulations were run utilizing a Newtonian, viscous model, with laminar flow conditions with the following fluid material properties: density $=1.01 \mathrm{~g} / \mathrm{cm}^{3}$ and dynamic viscosity $=$ 
$1.27 \mathrm{cp}$ which were previously measured for regular cell culture basal media (Ramaswamy et al., 2014). The outlet of the bioreactor was set to a zero relativepressure boundary condition. The housed bioreactor specimens were assumed to have a constant, homogeneous porosity that were saturated with cell culture medium. The cell density prescribed was $17 \times 10^{6} \mathrm{cells} / \mathrm{cm}^{2}$ (Engelmayr et al., 2006). The rectangular sample dimensions ( $L X W X t)$ were as follows: $17 \mathrm{~mm} X$ $6.5 \mathrm{~mm} \times 1 \mathrm{~mm}$ equal to a total sample surface area of $2.68 \mathrm{~cm}^{2}$. This represented a total number of cells of $4.556 \times 10^{7}$ in the surfaces on the rectangular samples. The results were analyzed after a convergence criteria of $1 \times 10^{-9}$ set for each of the momentum, continuity, mesh displacement, and mass transport equations were satisfied. Simulations were conducted in a Hewlett Packard work station with intel(R) Xeon(R) CPU, x5550@ 2.67GHz (2 processors) with 16.0 GB installed memory and 64-bit windows 7 operating system. 


\begin{tabular}{ll}
\hline Walls & No slip conditions \\
\hline Inlet & Plug velocity $0.02 \mathrm{~m} / \mathrm{s}$ \\
Outlet & Outlet-Relative Pressure = 0 Pa \\
Samples & Porous-fluid Interface \\
\hline
\end{tabular}

Table 2. Boundary conditions used for the CFD transport model

a)

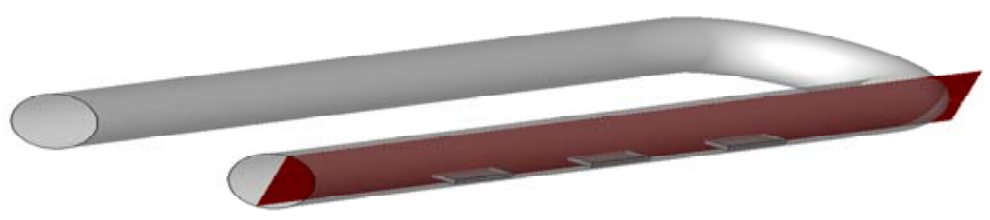

b)

c)

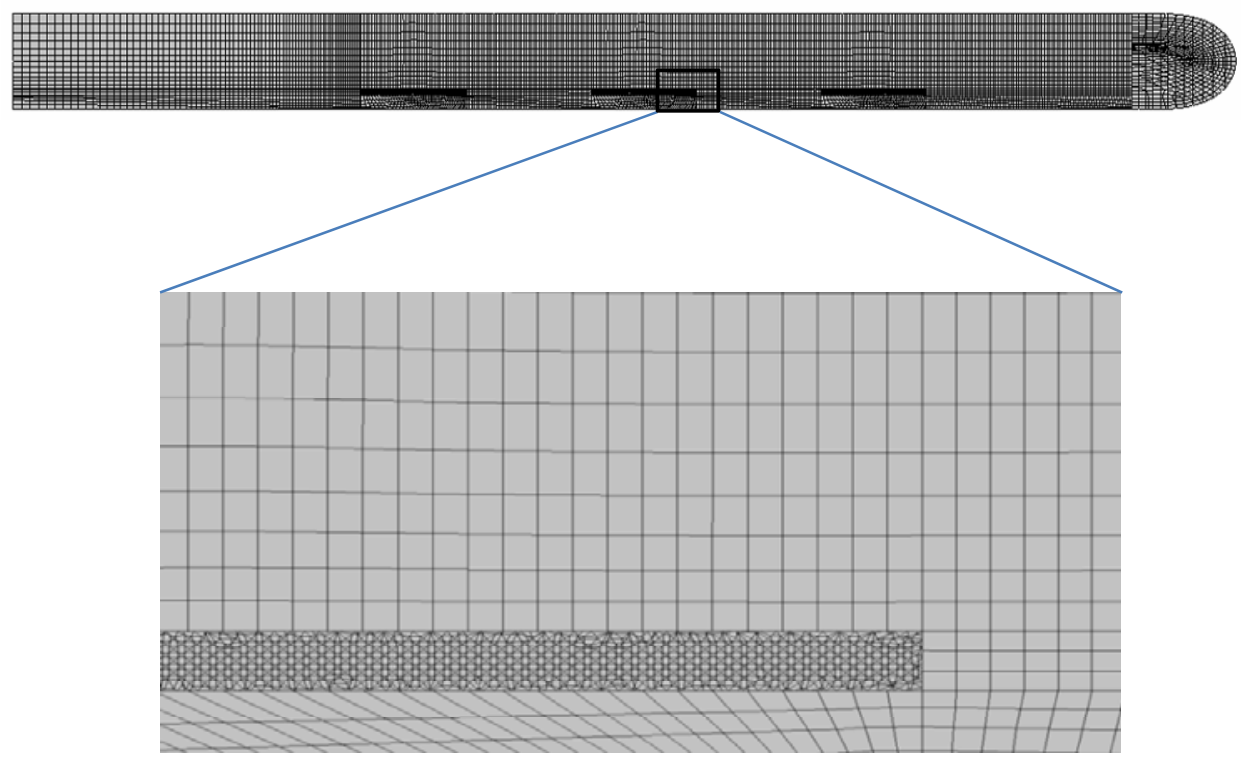

Figure 24: a) Depiction of the entire fluid domain of the bioreactor chamber. Note the three scaffolds in a straight configuration and the plane (in red) at $x=$ $0.0175 \mathrm{~m}$ corresponding to the longitudinal center line of the samples. b) Cross section $(x=0.0175 \mathrm{~m})$ of the mesh. Note that the element density is higher inside the samples in order to better capture mass transport events. c) A close-up of the mesh around a single sample. 


\subsection{Convective-diffusive model}

Here, we built on this system by coupling the momentum transport (Salinas and Ramaswamy, 2014) to a convective and diffusion mass transport model which can provide information on glucose and oxygen-species distribution within the housed bioreactor specimens over time and accounts for physiologically valid glucose and oxygen cell consumption rates. The model is characterized by the following equations:

$\Phi_{\mathrm{f}} \frac{\delta c^{\beta}}{\delta \mathrm{t}}+\left(\mathrm{v}_{\mathrm{f}} \gamma \mathrm{C}^{\beta}-\mathrm{D}^{\beta *} \nabla \mathrm{C}^{\beta}\right)=\mathbf{q}^{\beta}$

$\mathbf{D}^{\beta *}=\frac{\mathbf{v}_{\mathbf{f}}}{\left(2-\mathbf{v}_{\mathrm{f}}\right)^{2}} D^{\boldsymbol{\beta}}$ Mackie and Meares Relation $\quad \mathbf{K}_{\mathrm{m}}^{\beta} \ll \mathbf{C}^{\boldsymbol{\beta}}$

$\mathbf{q}^{\boldsymbol{\beta}}=\frac{\mathbf{v}^{\beta} \boldsymbol{\rho}^{\text {cell }}}{\mathbf{K}_{\mathrm{m}}^{\beta}+\mathbf{C}^{\beta}} \mathbf{C}^{\boldsymbol{\beta}} \quad$ Michaelis-Menten Relation $\quad \mathbf{q}^{\boldsymbol{\beta}}=-\mathbf{V}^{\boldsymbol{\beta}} \boldsymbol{\rho}^{\text {cell }}$

Where $C^{\beta}$ is the concentration of the solute in the medium; $\Phi_{\mathrm{f}}$ is the fluid volume fraction; $\gamma$ is the sieving coefficient in a porous material; $q^{\beta}$ is the source term for generation, consumption or degradation of solute mass; $\mathrm{v}_{\mathrm{f}}$ is the velocity of the fluid phase; $D^{\beta}$ is the effective diffusive coefficient in the porous medium; $\rho$ cell is the density of cell seeded in the scaffold; $\vee \beta$ is the maximum uptake rate, $K_{m}^{\beta}$ is the concentration at half velocity. The diffusivity in the fluid was prescribed to be $5.40 \times 10^{-6} \mathrm{~cm}^{2} / \mathrm{s}$ for glucose and $5.40 \times 10^{-6} \mathrm{~cm}^{2} / \mathrm{s}$ for oxygen (Das, 2007).

Meanwhile, the diffusivity in the scaffold was set to be $1.08 \times 10^{-6} \mathrm{~cm}^{2} / \mathrm{s}$ and $0.60 \times 10^{-5} \mathrm{~cm}^{2} / \mathrm{s}$ for glucose and oxygen respectively (Das, 2007). Finally, the glucose consumption rate was set to $3.83 \times 10^{-16}$ mole/cell.s, while oxygen was assumed to be consumed at a rate of $3.75 \times 10^{-17}$ mole/cell.s (Das, 2007). 


\begin{tabular}{lll}
\hline Property & Glucose & Oxygen \\
\hline Diffusivity in Fluid $\mathrm{cm}^{2} / \mathrm{s}$ & $5.40 \times 10^{-6}$ & $3.00 \times 10^{-5}$ \\
Diffusivity in scafffold $\mathrm{cm}^{2} / \mathrm{s}$ & $1.08 \times 10^{-6}$ & $0.60 \times 10^{-5}$ \\
\hline Metabolism rate in cell mole/cell.s & $3.83 \times 10^{-16}$ & $3.75 \times 10^{-17}$ \\
Metabolism rate in cell grams/cell.s & $6.90 \times 10^{-14}$ & $6.00 \times 10^{-16}$ \\
\hline Total Metabolism rate in sample g/s & $-3 \times 10^{-06}$ & $-2.7335 \times 10^{-08}$ \\
\hline
\end{tabular}

Table 3. Parameters used in the mass transport model.

\subsection{Porosity and Mass Fraction}

In order to determine the porosity of the PLA/PGA scaffold we first measured its dry mass $\left(\mathrm{W}_{\mathrm{S}}\right)$. Then, the scaffold was submerged in water. After that we recorded the weight of the water and the scaffold $\left(\mathrm{W}_{\mathrm{G}}\right)$. The weight of the residual water $\left(\mathrm{W}_{\mathrm{GI}}\right)$ was also recorded after the scaffold was removed from the container.

The void volume was given by $V_{V}=\frac{W_{G}-2 W_{S}-W_{G l}}{\rho_{W}}$ and the total volume was $V_{T}=\frac{W_{G}-W_{S}-W_{G l}}{\rho_{w}}$. Where $\rho_{w}$ is the density of water; the porosity of the sample was given by $\frac{V_{V}}{V_{T}} * 100$. The porosity of the scaffold before any cell culture experiments was $\sim 95 \%$. After the cell culture experiments the scaffold showed a porosity of $75 \%$. Glucose and Oxygen mass fractions in media were computed based on the glucose concentration in cell culture media (DMEM, fisher sci, pittbsurgh, PA) and from the ideal gas law respecitvely. The mass fraction of glucose was found to be 0.0045 , whereas for oxygen it was 0.00013 . 


\subsection{Results}

As observed in Figures 25-29, the flow physics surrounding the engineered tissue constructs resembled those previously described in (Salinas and Ramaswamy, 2014). Briefly, flow was unidirectional during steady flow conditions acting on straight specimens and minor flow changes were observed on cases of steady flow and sample semi bent and fully bent configurations. Flow vortices were observed at the beginning of the bending cycle in cases of cyclic flexure-alone and they showed displacement and size changes at subsequent time steps. Similar events where observed during conditions of flow and sample flexure but with lower magnitude of events.

\section{Flex-flow}

$$
\begin{aligned}
& t=0 \mathrm{~s} \\
& t=0.25 \mathrm{~s} \\
& t=0.5 \mathrm{~s} \\
& t=0.75 \mathrm{~s} \\
& t=1 \mathrm{~s}
\end{aligned}
$$
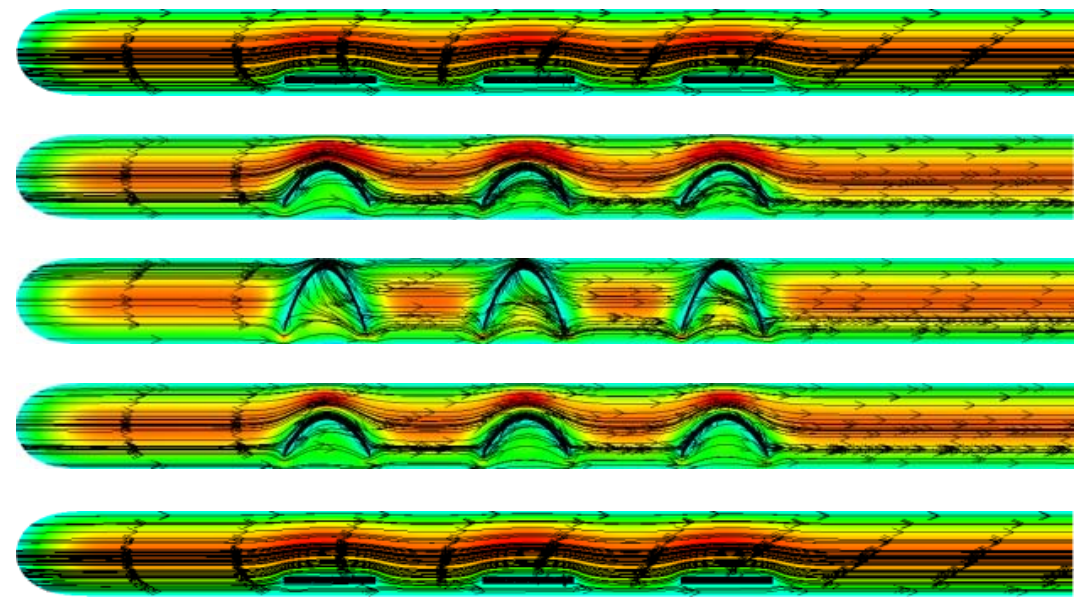

Flow direction

Axial velocity $(\mathrm{m} / \mathrm{s})$

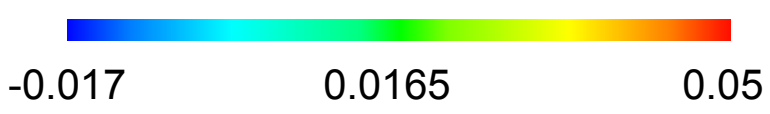

Figure 25. Contour of velocity magnitudes and velocity streamlines in the plane $(x=17.5 \mathrm{~mm})$ surrounding the specimens during flex-flow states 
Cyclic flexure-alone

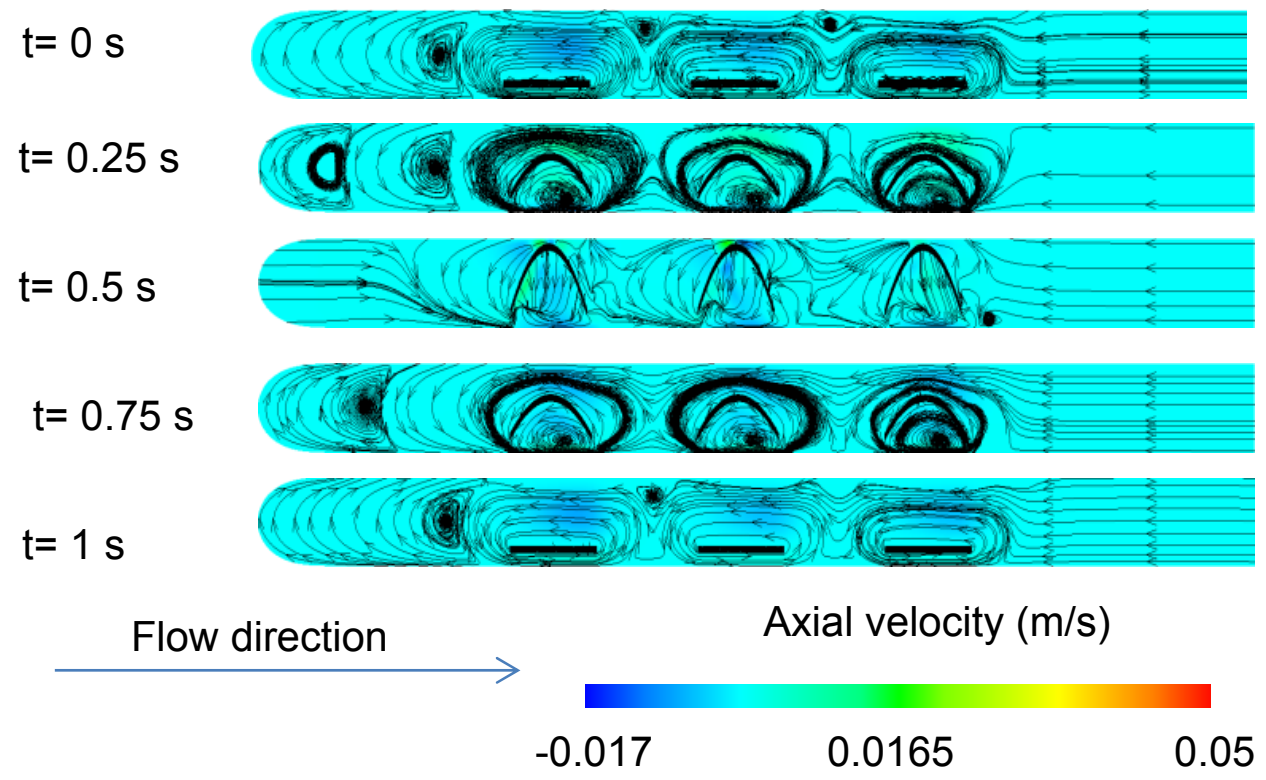

Figure 26. Contour of velocity magnitudes and velocity streamlines in the plane $(x=17.5 \mathrm{~mm})$ surrounding the specimens during cyclic flexure-alone states.

Steady flow-alone straight

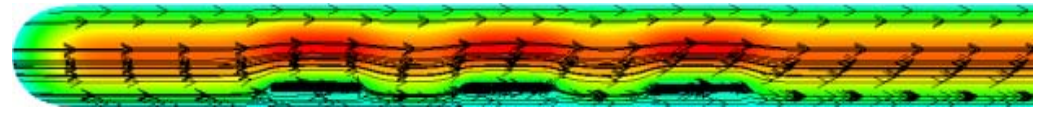

Steady flow-alone semi-bent

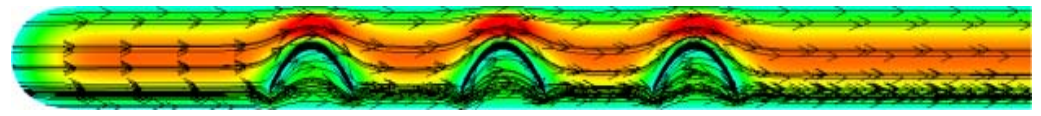

Steady flow-alone fully-bent
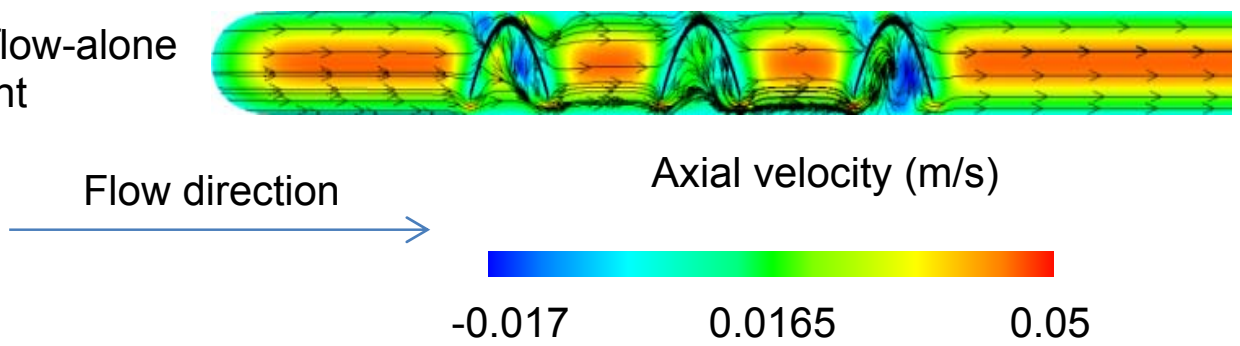

Figure 27. Contour of velocity magnitudes and velocity streamlines in the plane $(x=17.5 \mathrm{~mm})$ surrounding the specimens during steady flow-alone states. 


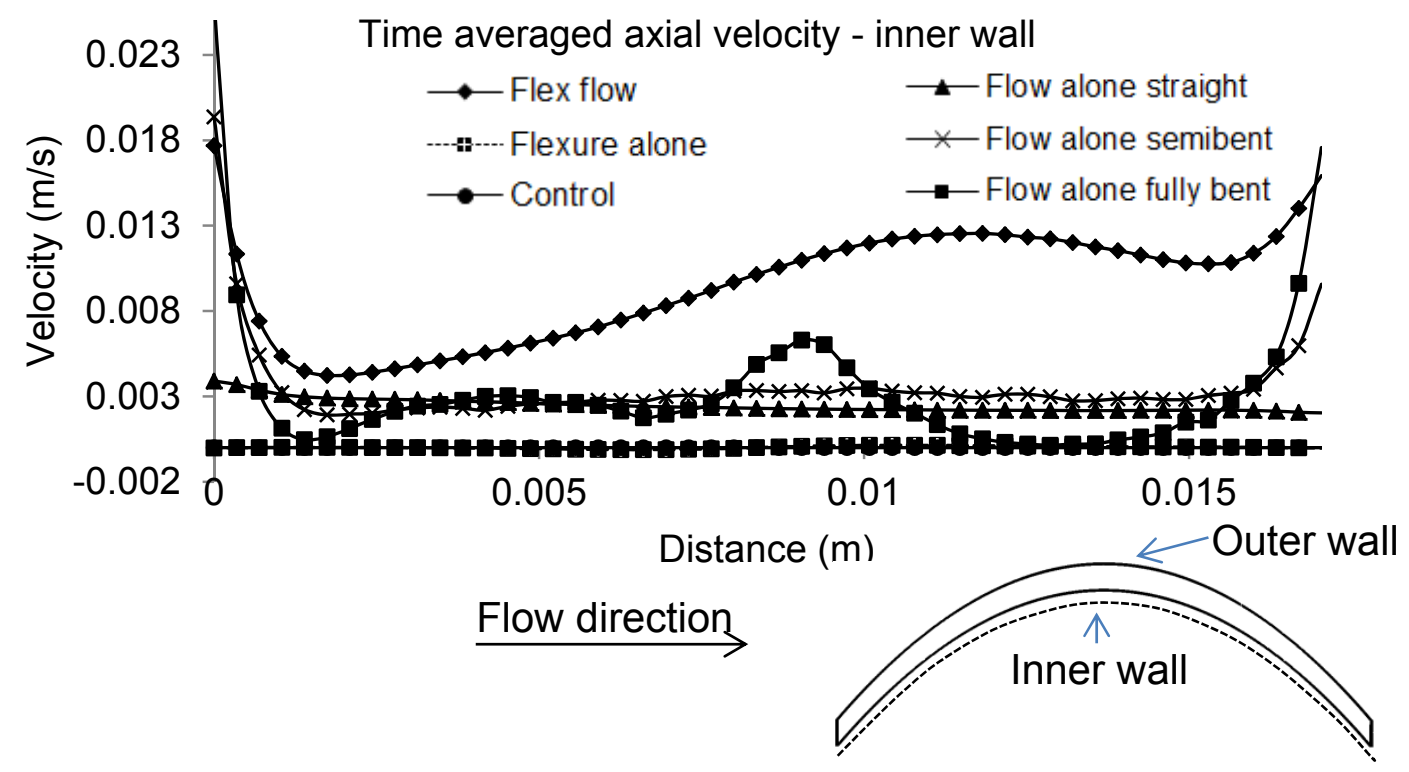

Figure 28. Time averaged axial velocities (plane $x=-0.0175 \mathrm{~m}$ ) from line at $0.00015 \mathrm{~m}$ below the inner wall.

Time averaged axial velocity - outer wall

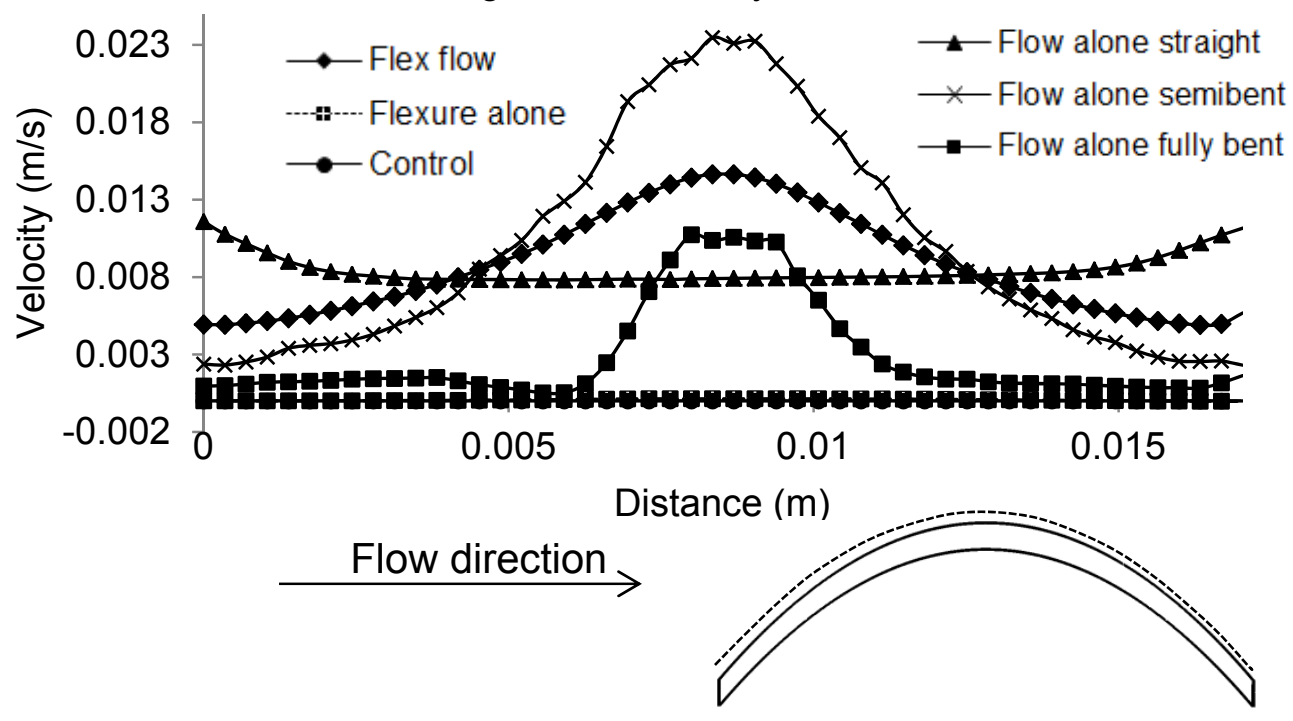

Figure 29. Time averaged axial velocities (plane $x=-0.0175 \mathrm{~m}$ ) from line at $0.00015 \mathrm{~m}$ above the outer wall. 
Normalized mass fractions of glucose and oxygen were high at high porosities as seen in Figures 30-31. This is the norm for samples with porosity greater than forty percent. Approximately at forty percent porosity the mass fractions begin to decrease, and it is significantly lower at low sample porosities such as ten or five percent. There is no presence of mass fraction in samples that are fully solid (no porosity).

At $75 \%$ porosity levels, there was no significant glucose mass fraction differences among cases of flex-flow, cyclic flexure-alone, flow-alone, and control. It can be observed in Figure 32 that the glucose mass fraction ranged from 0.967 to 0.991 ; analysis of variances shows no differences among groups at a significant level of $p=0.05$. The oxygen mass fraction ranged from 0.978 to 0.999 as shown in Figure 33. Analysis of variances shows no differences among groups at a significant level of $p=0.05$.

Normalized mass fraction contour along the center of the sample are shown in Figure 34. It can be seen that in the case of glucose, there is good uniformity in mass distribution along the center of the sample matrix. There is highest presence of glucose in the flex-flow case, followed by the cyclic flexurealone case. Next were the flow cases which showed very similar mass profiles among them. The lowest presence of mass was seen in the control group. Similar scenarios were observed for the normalized mass transport of oxygen as seen in Figure 35.

At sample porosities of $5 \%$, there was significant glucose mass fraction differences among cases of flex-flow, cyclic flexure-alone, flow-alone, and 
control. It can be observed in Figure 36 that the glucose mass fraction ranges from 0.144 to 0.002 ; analysis of variances revealed that all groups are different from each other at a significant level of $p=0.05$ with the exception that the flowalone groups did not show significant differences among them. The oxygen mass fraction ranges from 0.224 to 0.003 as seen in Figure 37 . Analysis of variances revealed that all groups are different from each other at a significant level of $p=0.05$ with the exception that the flow-alone groups did not show significant differences among them.

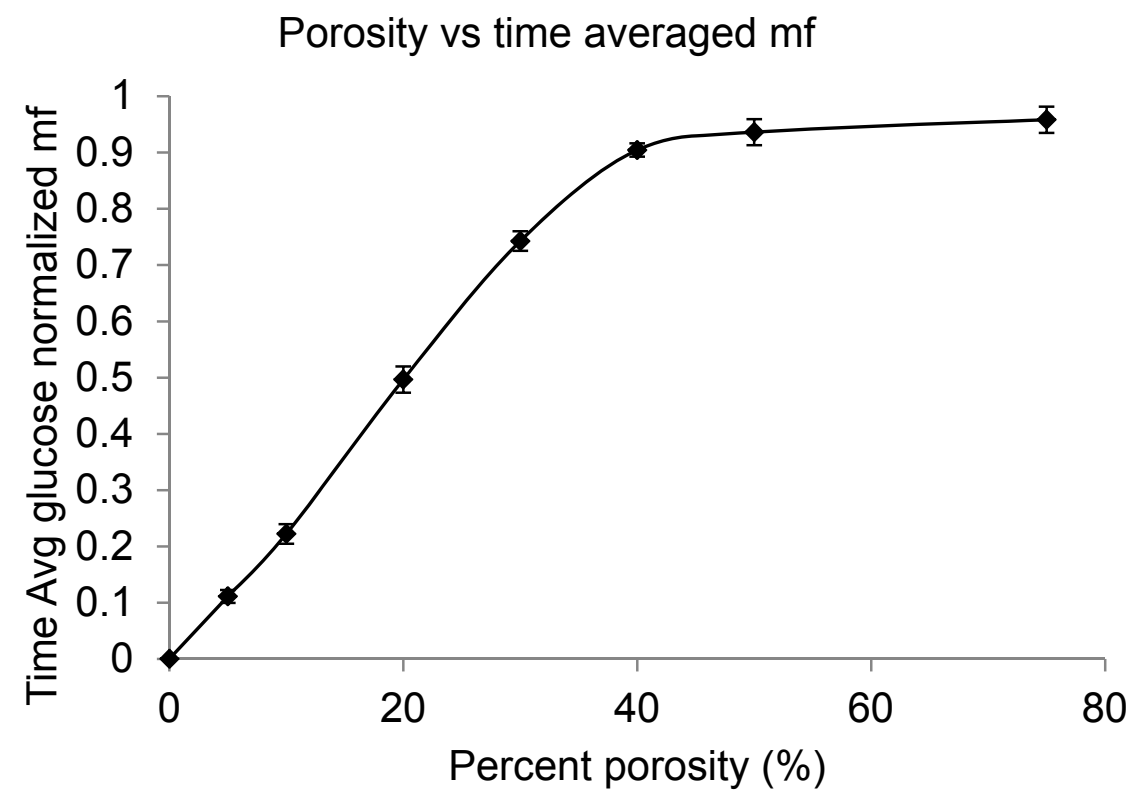

Figure 30. Time averaged normalized mass fraction for glucose at different porosity percentages. 


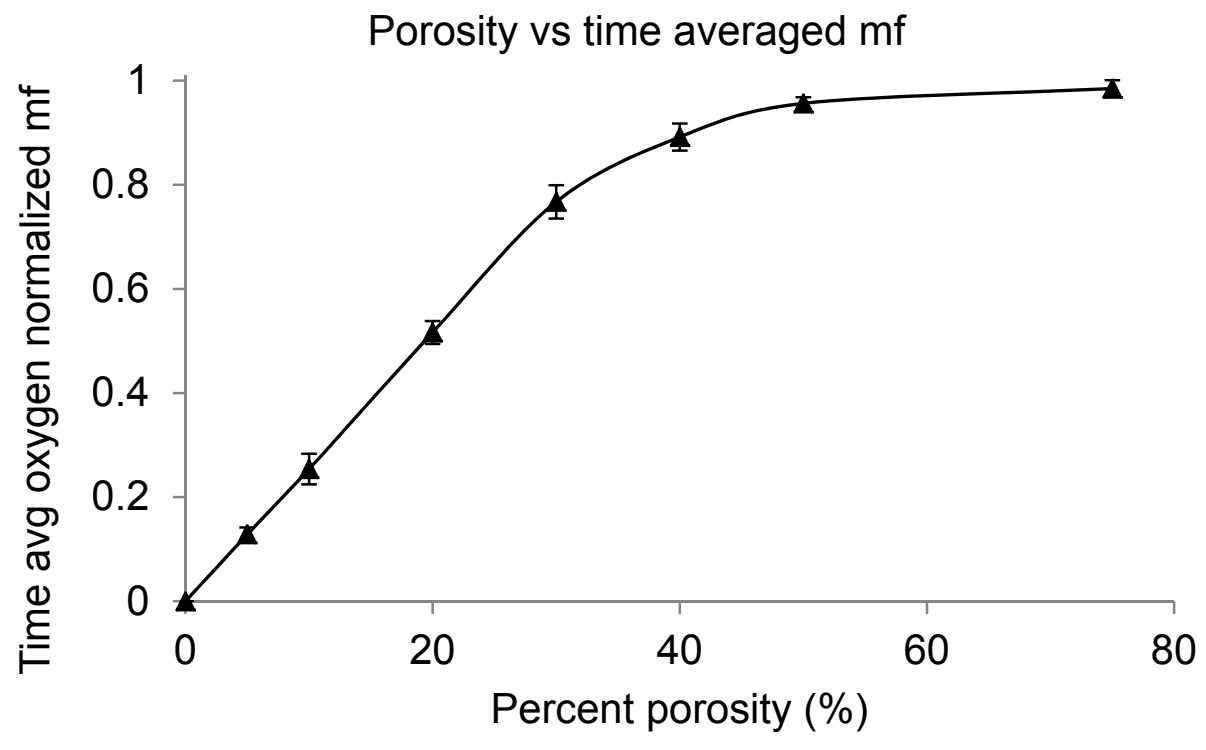

Figure 31. Time averaged normalized mass fraction for oxygen at different porosity percentages.

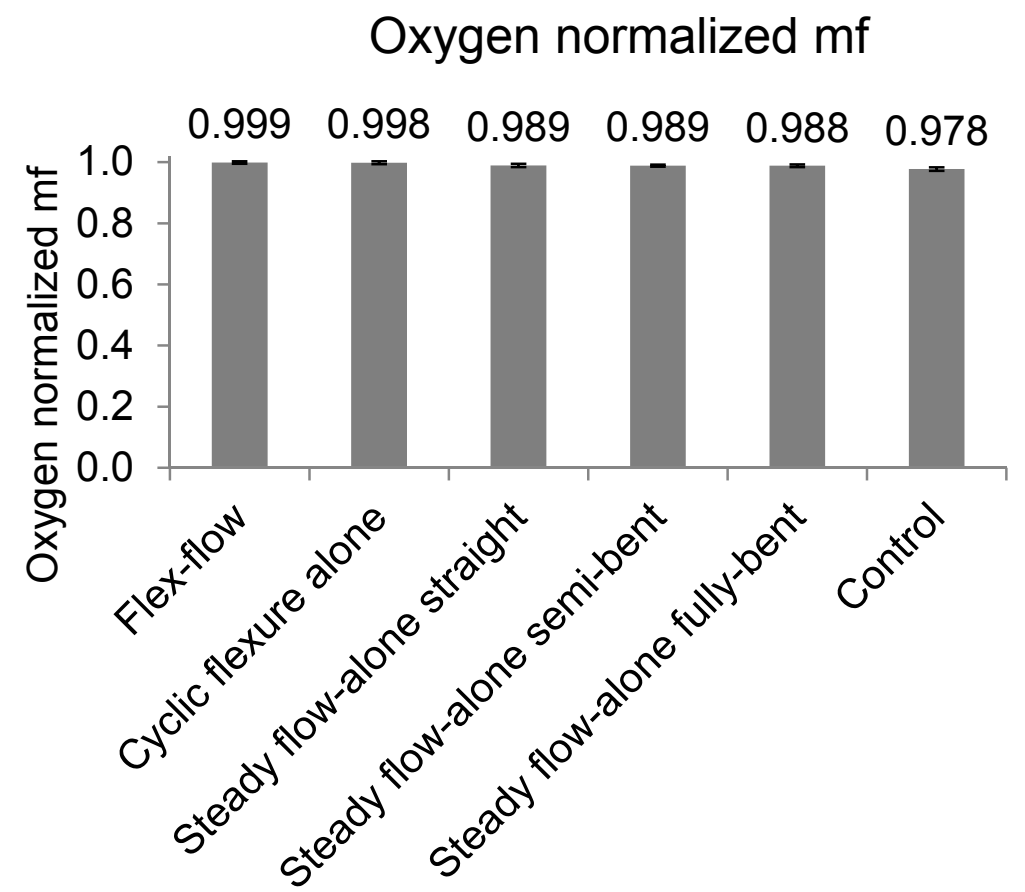

Figure 32 . At $75 \%$ porosity: Area and time averaged normalized mass fraction at sample longitudinal wall at $x=-0.0175 m$ for glucose. 


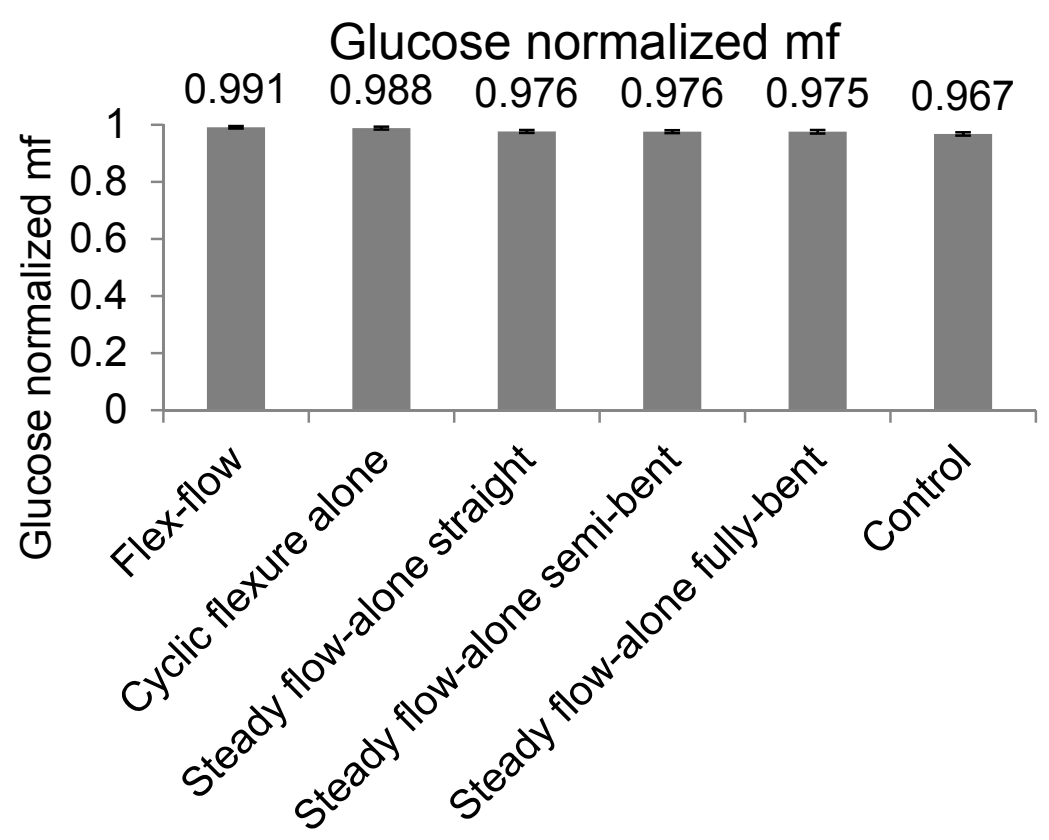

Figure 33 . At $75 \%$ porosity: Area and time averaged normalized mass fraction at sample longitudinal wall at $x=-0.0175 m$ for oxygen.

Center wall

Flex-flow

Cyclic flexure-alone

Steady flow-alone straight

Steady flow-alone semi-bent

Steady flow-alone fully-bent

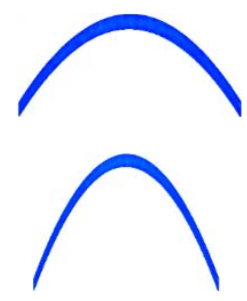

Control

Figure 34 . At $5 \%$ porosity: Time averaged normalized glucose mf (sample longitudinal wall at $x=-0.0175 \mathrm{~m}$ ) for cases of flex-flow, cyclic flexure-alone, steady flow-alone and control.
Center wall (plane at $\mathrm{x}=-0.175 \mathrm{~m})$

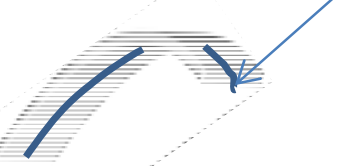


Flex-flow

Cyclic flexure-alone

Steady flow-alone straight

Steady flow-alone semi-bent

Steady flow-alone fully-bent

\section{Center wall}
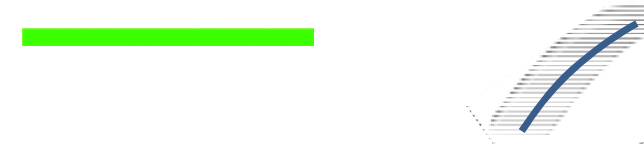

0.5 $\begin{array}{ll}0 & 0.25\end{array}$
Flow direction

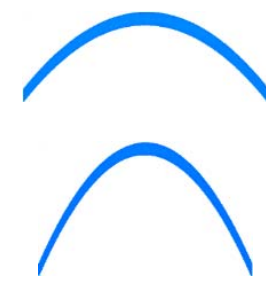

Time averaged normalized oxygen mass fraction

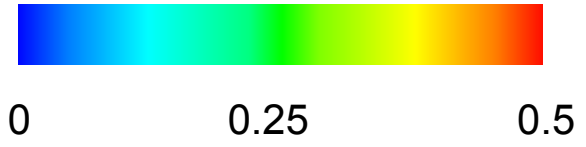

Control

Figure 35. Time averaged normalized oxygen $\mathrm{mf}$ (sample longitudinal wall at $\mathrm{x}=$ $-0.0175 \mathrm{~m}$ ) for cases of flex-flow, cyclic flexure-alone, flow-alone and control.

Glucose normalized mf

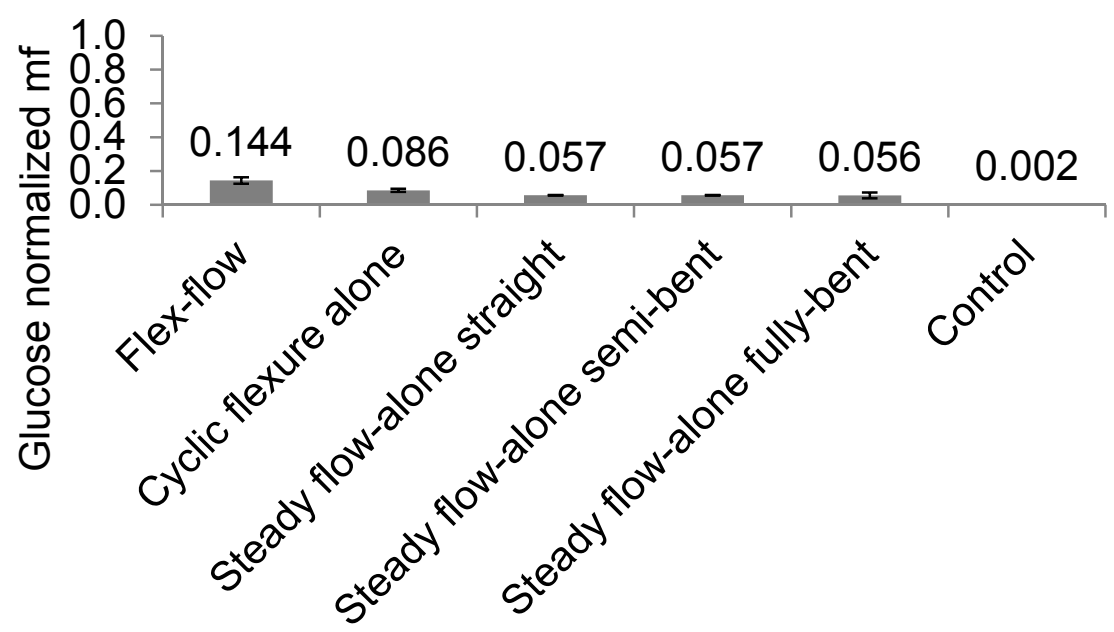

Figure 36. At 5\% porosity: Area and time averaged normalized mass fraction at sample longitudinal wall at $x=-0.0175 m$ for glucose . 


\section{Oxygen normalized $\mathrm{mf}$}

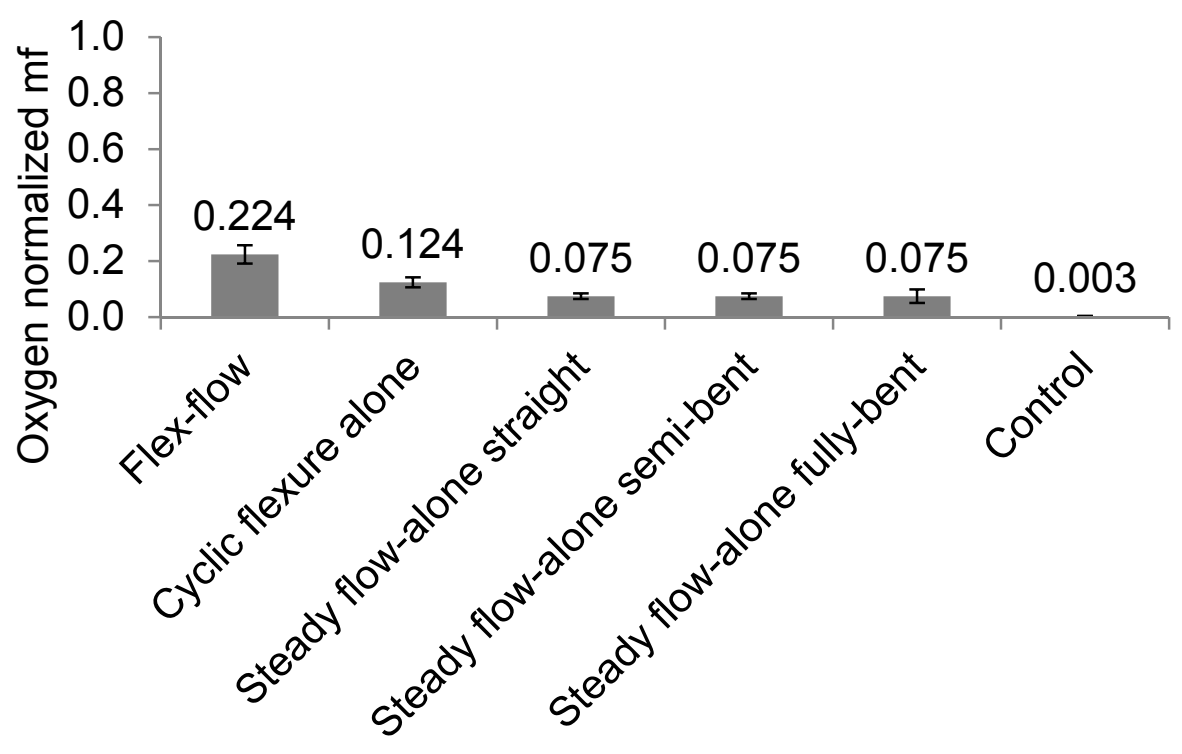

Figure 37 . At $5 \%$ porosity: Area and time averaged normalized mass fraction at sample longitudinal wall at $x=-0.0175 m$ for oxygen.

ANOVA
\begin{tabular}{|l|r|r|r|r|r|}
\hline & \multicolumn{1}{c|}{ Sum of } & df & Mean Square & F & Sig. \\
\hline Squares & .001 & 5 & .000 & 2.298 & .110 \\
Within Groups & .001 & 12 & .000 & & \\
Total & .002 & 17 & & & \\
\hline
\end{tabular}

Table 4. At 75\% porosity: Anova results for normalized glucose.

ANOVA

Normalized oxygen

\begin{tabular}{|l|r|r|r|r|r|}
\hline & \multicolumn{1}{|c|}{$\begin{array}{c}\text { Sum of } \\
\text { Squares }\end{array}$} & df & Mean Square & F & Sig. \\
\hline Between Groups & .001 & 5 & .000 & 1.379 & .299 \\
Within Groups & .001 & 12 & .000 & & \\
Total & .002 & 17 & & & \\
\hline
\end{tabular}

Table 5. At 75\% porosity: Anova results for normalized glucose. 


Normalized glucose
\begin{tabular}{|l|r|r|r|r|r|}
\hline & \multicolumn{1}{c|}{ Sum of } \\
& Squares & df & Mean Square & F & Sig. \\
\hline Between Groups & .032 & 5 & .006 & 642.116 & .000 \\
Within Groups & .000 & 12 & .000 & & \\
Total & .033 & 17 & & & \\
\hline
\end{tabular}

Table 6 . At 5\% porosity: Anova results for normalized glucose.

\section{Multiple Comparisons}

Dependent Variable: Normalized glucose

Tukey HSD

\begin{tabular}{|c|c|c|c|c|}
\hline (I) Case & (J) Case & $\begin{array}{c}\text { Mean } \\
\text { Difference (I-J) }\end{array}$ & Std. Error & Sig. \\
\hline \multirow{6}{*}{ flex-flow } & Cyclic flexure & .0583826663 & .0025959427 & .000 \\
\hline & steady flo & $.0868963553^{*}$ & .0025959427 & .000 \\
\hline & steady flow-alone semi-bent & $.0870845327^{*}$ & .0025959427 & .000 \\
\hline & steady flow-alone fully-bent & $.0879335993^{x}$ & .0025959427 & .000 \\
\hline & control & $.1419857773^{x}$ & .0025959427 & .000 \\
\hline & flex- & $-.0583826663^{x}$ & .0025959427 & .000 \\
\hline \multirow{5}{*}{$\begin{array}{l}\text { Cyclic flexure- } \\
\text { alone }\end{array}$} & steady flow-a & $.0285136890^{*}$ & .0025959427 & .000 \\
\hline & steady flow-alone semi-bent & $.0287018663^{*}$ & .0025959427 & .000 \\
\hline & steady flow-alone fully-bent & $.0295509330^{*}$ & .0025959427 & .000 \\
\hline & control & .0836031110 & .0025959427 & .000 \\
\hline & flex-flow & -.0868963553 & .0025959427 & .000 \\
\hline \multirow{4}{*}{$\begin{array}{l}\text { steady flow-alone } \\
\text { straight }\end{array}$} & Cyclic flexur & $-.0285136890^{*}$ & .0025959427 & .000 \\
\hline & steady flow & .0001881773 & .0025959427 & 1.000 \\
\hline & steady flow-alone fully-bent & .0010372440 & .0025959427 & .998 \\
\hline & $\begin{array}{l}\text { control } \\
\text { flex-flow }\end{array}$ & $\begin{aligned} .0550894220 \\
-.0870845327\end{aligned}$ & $\begin{array}{l}.0025959427 \\
.0025959427\end{array}$ & $\begin{array}{l}.000 \\
000\end{array}$ \\
\hline \multirow{5}{*}{$\begin{array}{l}\text { steady flow-alone } \\
\text { semi-bent }\end{array}$} & Cyclic flexu & $-.0287018663^{*}$ & .0025959427 & .000 \\
\hline & steady flow & -.0001881773 & .0025959427 & 1.000 \\
\hline & lone fully-bent & .0008490667 & 59427 & .999 \\
\hline & control & $.0549012447^{*}$ & 59427 & .000 \\
\hline & flex-flow & -.0879335993 & .0025959427 & .000 \\
\hline \multirow{5}{*}{$\begin{array}{l}\text { steady flow-alone } \\
\text { fully-bent }\end{array}$} & Cyclic flexu & $-.0295509330^{*}$ & .0025959427 & .000 \\
\hline & flon & -.0010372440 & .0025 & .998 \\
\hline & eady flow & 190667 & .0025959427 & .999 \\
\hline & & $\begin{array}{r}.0540521 / 80 \\
-.1419857773\end{array}$ & $\begin{array}{l}.0025959427 \\
.0025959427\end{array}$ & .000 \\
\hline & Cyclic flexure-alone & $-.0836031110^{*}$ & .0025959427 & .000 \\
\hline \multirow[t]{3}{*}{ control } & steady flow-alone str & $-.0550894220^{\pi}$ & .0025959427 & .000 \\
\hline & steady flow-alone semi-b & $-.0549012447^{x}$ & .0025959427 & .000 \\
\hline & steady flow-alone fully-bent & $-.0540521780^{x}$ & .0025959427 & .000 \\
\hline
\end{tabular}

Table 7. At $5 \%$ porosity: Multiple comparisons using Tukey's test for normalized glucose. 
ANOVA

Normalized oxygen

\begin{tabular}{|l|r|r|r|r|r|}
\hline & \multicolumn{1}{|c|}{$\begin{array}{c}\text { Sum of } \\
\text { Squares }\end{array}$} & df & Mean Square & F & Sig. \\
\hline Between Groups & .082 & 5 & .016 & 55.448 & .000 \\
Within Groups & .004 & 12 & .000 & & \\
Total & .085 & 17 & & & \\
\hline
\end{tabular}

Table 8. At 5\% porosity: Anova results for normalized oxygen.

\section{Multiple Comparisons}

Dependent Variable: Normalized oxygen

Tukey HSD

\begin{tabular}{|c|c|c|c|c|}
\hline (I) Case & (J) Case & $\begin{array}{c}\text { Mean } \\
\text { Difference (I- } \\
\mathrm{J})\end{array}$ & Std. Error & Sig. \\
\hline \multirow{6}{*}{ flex-flow } & Cyclic flexure-alone & $.1000000000^{*}$ & .0140037338 & .000 \\
\hline & steady flow-alone straight & $.1494511033^{*}$ & .0140037338 & .000 \\
\hline & steady flow-alone semi-bent & $.1494511033^{*}$ & .0140037338 & .000 \\
\hline & steady flow -alone fully-bent & $.1494511033^{*}$ & .0140037338 & .000 \\
\hline & control & $.2209281770^{*}$ & .0140037338 & .000 \\
\hline & flex-flow & $-.1000000000^{*}$ & .0140037338 & .000 \\
\hline \multirow{5}{*}{$\begin{array}{l}\text { Cyclic flexure- } \\
\text { alone }\end{array}$} & steady flow-alone straight & $.0494511033^{*}$ & .0140037338 & .037 \\
\hline & steady flow-alone semi-bent & $.0494511033^{*}$ & .0140037338 & .037 \\
\hline & steady flow-alone fully-bent & $.0494511033^{*}$ & .0140037338 & .037 \\
\hline & control & $1209281770^{*}$ & .0140037338 & .000 \\
\hline & flex-flow & $-.1494511033^{*}$ & .0140037338 & .000 \\
\hline \multirow{5}{*}{$\begin{array}{l}\text { steady flow-alone } \\
\text { straight }\end{array}$} & Cyclic flexure-alone & $-.0494511033^{*}$ & .0140037338 & .037 \\
\hline & steady flow-alone semi-bent & .0000000000 & .0140037338 & 1.000 \\
\hline & steady flow-alone fully-bent & .0000000000 & .0140037338 & 1.000 \\
\hline & control & $.0714770737^{*}$ & .0140037338 & .003 \\
\hline & flex-flow & $-.1494511033^{*}$ & .0140037338 & .000 \\
\hline \multirow{4}{*}{$\begin{array}{l}\text { steady flow-alone } \\
\text { semi-bent }\end{array}$} & Cyclic flexure-alone & $-.0494511033^{*}$ & .0140037338 & .037 \\
\hline & steady flow -alone straight & .0000000000 & .0140037338 & 1.000 \\
\hline & steady flow-alone fully-bent & .0000000000 & .0140037338 & 1.000 \\
\hline & control & $.0714770737^{*}$ & .01400 & .003 \\
\hline steady flow -alone & flex-flow & $-.1494511033^{*}$ & .0140037338 & .000 \\
\hline
\end{tabular}




\begin{tabular}{|ll|r|r|r|} 
fully-bent & Cyclic flexure-alone & $-.0494511033^{*}$ & .0140037338 & .037 \\
& steady flow-alone straight & .0000000000 & .0140037338 & 1.000 \\
& steady flow-alone semi-bent & .0000000000 & .0140037338 & 1.000 \\
& control & $.0714770737^{*}$ & .0140037338 & .003 \\
& flex-flow & $-.2209281770^{*}$ & .0140037338 & .000 \\
& Cyclic flexure-alone & $-.1209281770^{*}$ & .0140037338 & .000 \\
control & steady flow -alone straight & $-.0714770737^{*}$ & .0140037338 & .003 \\
& steady flow-alone semi-bent & $-.0714770737^{*}$ & .0140037338 & .003 \\
& steady flow-alone fully-bent & $-.0714770737^{*}$ & .0140037338 & .003 \\
\hline
\end{tabular}

Table 9. At 5\% porosity: Multiple comparisons using Tukey's test for normalized oxygen.

\subsection{Discussion}

Previously, we computationally investigated the effects of sample movement on the flow physics surrounding tissue engineered constructs (Salinas and Ramaswamy, 2014). We virtually replicated the experimental set up of a particularly relevant in vitro heart valve tissue engineering experiment conducted by Engelmayr et al. (Engelmayr et al., 2006) where rectangular PLA/PGA nonwoven, fibrous scaffolds seeded with BMSCs where mechanically conditioned in a FSF bioreactor. They showed that cyclic specimen flexure combined with a steady flow source greatly augmented engineered collagen production when compared to cases of cyclic flexure-alone or steady flow-alone. Our previous computational findings (Salinas and Ramaswamy, 2014) led us to note that a combination of shear stress magnitude and oscillatory shear stress directly correlated with collagen augmentation while each parameter on its own could not explain greater tissue formation. In addition, sample flexure may have enhanced nutrient delivery of glucose and oxygen through the walls of engineered 
constructs and into the cells and tissues thereby adding in the process of tissue production.

In this study, we sought to computationally determine if, in addition to OSI$\overrightarrow{|\tau|}$ (Salinas and Ramaswamy, 2014), mass transport of glucose and oxygen played a role in augmented tissue formation reported in the in vitro flex-flow case (Engelmayr et al., 2006). We incorporated a convective-diffusive mass transport model (Das, 2007; Sengers et al., 2005) to our previously validated CFD simulations where we looked at cases of flex-flow, cyclic flexure-alone, flowalone and controls. We elected this mass transport model because it provided with mass concentration of glucose and oxygen species resulting from convective and diffusive activity over time anywhere in the bioreactor and it used physiologically valid cell consumption rates of both species.

Tissue culture and mathematical based studies have clearly established the importance of adequate oxygen (Kumar et al., 2004; Wang et al., 2008) and glucose delivery (Boubriak et al., 2006; Converti et al., 1996; Mokhbi Soukane et al., 2007) for adequate cell activity and tissue formation and noted that any unmet demand of nutrients can lead to low cell proliferation, and low collagen production particularly at the center of scaffolds with low porosities (Ishaug-Riley et al., 1998; Patnaik et al., 2013).

Some studies have reported that scaffolds used for tissue engineering experiments have porosity as low as $73 \%$ (Yu et al., 2009) after cell and tissue culture. During an in-house in vitro experiment, we determined that the porosity of the 50:50 PLA/PGA scaffold was $95 \%$ before culture and $75 \%$ porous after 
tissue culture experiments. Our simulations showed that there is adequate delivery of both glucose and oxygen at high specimen porosities ranging from forty percent porosity and higher (Figure 30-31). We confirmed this observation by performing an anova test which showed no difference in the mass fraction of glucose and oxygen during cases of flow, flexure and both combined. These results suggest that sample cyclic flexure does not enhance nutrient delivery to the center of engineered tissue constructs because diffusivity effects dominate when scaffolds have high porosities, which is usually the norm for tissue engineering studies that started tissue culture experiments with a highly porous specimen (Yu et al., 2009). We note however that at low sample porosities (5\%), our simulations do show that sample movement enhanced nutrient delivery as shown in figures 34-37 and validated by an anova study (Table 8-9). Although low porosities are unlikely to be seen in tissue engineering studies, we acknowledge that in some cases such as using decellularized scaffolds with high ECM content low porosities can be observed and sample movement should be highly considered in order to enhance nutrient transport. In addition, studies using scaffolds with high thickness such as whole organ development may also benefit from scaffold bending modes.

\section{CHAPTER 6: PRELIMINARY EXPERIMENTAL OBSERVATIONS FOR OSI-| $\vec{\tau} \mid$}

\subsection{Introduction}

We have computationally shown by an initial approximation given as OSI-

$\overrightarrow{|\tau|}$ that both OSI and TSSM combined correlated with significantly higher 
engineered collagen production during modes of sample cyclic flexure combined with a steady flow source. The OSI-| $\overrightarrow{\tau \mid}$ was defined as twice the product of the TSSM and the OSI. In previously resolved simulations (Salinas et al., 2014a; Salinas et al., 2014b), we noted that oscillatory shear environments can be maximized by a pulsatile flow source passing by straight static specimens. We believe that the OSI- $\overrightarrow{|\tau|}$ parameter is also present in significant magnitude when flat specimens are subjected to physiologically valid pulsatile flow conditions and that OSI- $\overrightarrow{|\tau|}$ is responsible for inducing higher cell proliferation, gene expression and tissue formation. In this study, we performed 2D in vitro cell culture experiments where we subjected BMSCs seeded in straight surfaces to conditions of physiologically valid pulsatile flow conditions and steady state flow conditions. We conducted cell proliferation and gene expression assays and determined if pulsatility environments enhanced these traits. In addition, we performed a CFD simulation that replicated this in vitro experiment in order to delineate and quantify OSI, TSSM, and OSI-| $\overrightarrow{|\tau|}$ parameters. Finally, we wanted to correlate these parameters with results from the cell proliferation and gene expression assays.

\subsection{Methodology for Cell Culture experiments}

6.2.1 Cell Differentiation: BMSCs were cultivated in T-75 flasks in a $37^{\circ} \mathrm{C}$ incubator until $100 \%$ confluence was obtained, and were subsequently divided to obtain a cell density of $17 \times 10^{6} \mathrm{cell} / \mathrm{s} / \mathrm{cm}^{2}$ and cultured for an additional week. The no flow (control) group ( $\mathrm{n}=4$ ) was cultured in the Bioflux 200 system (Fluxion 
Biosciences, San Francisco, CA) system without any flow sources. The steady flow group ( $n=4$ ) was cultured in the Bioflux 200 system and exposed to a steady flow source capable of inducing an averaged shear stress of $1.5 \mathrm{dyn} / \mathrm{cm}^{2}$. The pulsatile flow group was cultured in the Bioflux 200 system and exposed to a pulsatile shear stress obtained from a physiological pulmonary artery waveform (Lotz et al., 2002).

6.2.2 Culture Media: The culture media contained DMEM High glucose (Invitrogen, Carlsbald, CA), 10\% fetal bovine serum (ATCC, Manasas, VA), 1\% antibiotic/antimycotic, 1\% HEPES (Invitrogen, Carlsbald, CA), $2 \mathrm{ng} / \mathrm{mL}$ of basic fibroblast growth factor (bFGF, Invitrogen, Carlsbald, CA), $82 \mu \mathrm{g} / \mathrm{mL}$ Ascorbic Acid (Sigma-Aldrich, St. Louis, MO). Media changes were performed every 24 hours for a period of one week. The amount of media added to each well for the 24-microfluidic well plate in the bioflux system was $3 \mathrm{ml}$ for the 96-well plate was $200 \mu \mathrm{L}$ for the controlgroup. The media contained $82 \mu \mathrm{g} / \mathrm{mL}$ of Ascorbic acid.

6.2.3 Bioflux 200 System: The Bioflux 200 system comprises of a pneumatic pump, a 24-microfluidic well plate, a 24-well plate interface, and a user operated software. It permits mechanical conditioning of rectangular channels seeded with cells by inducing steady or pulsatile flow conditions The Bioflux 200 is connected to a microscope with a CCD camera allowing the user to take photographs of the cultured cells under flow. This system is efficient for stem cell applications, such as cell differentiation and gene expression. 


\subsubsection{Assays:}

Cell Differentiation-RNA Isolation and Reverse Transcription: SMCs were washed with PBS and detached from the T-75 $\mathrm{cm}^{2}$ flask (Fisher Scientific, PA) by 0.25\%Trypsin-EDTA (ATCC, Manasas, VA). Then, the detached cells were collected in a $15 \mathrm{ml}$ conical tube (Fisher Scientific, PA). For the RNA isolation procedure, the cells were transferred to a $1.5 \mathrm{ml}$ RNAase free tube. Purification of mRNA was performed through the SV Total RNA Isolation System (Promega, Madison, $\mathrm{WI})$. We conducted Reverse transcription reaction on $1 \mu \mathrm{g}$ of total mRNA with GoScript ${ }^{\mathrm{TM}}$ Reverse Transcription System (Promega, Madison, WI). The detection process was achieved through a Step One Real-Time PCR System (Applied Biosystems, Carlsbad, CA). The cycling parameters were 950C, 5 seconds; 600C, 45 seconds; 950C, 15 seconds.

\subsubsection{CFD Simulations}

Using Ansys ${ }^{\mathrm{TM}}$ commercial software (Ansys Inc, Canonsburg, PA), the fluid domain of the rectangular bioflux channel was constructed. The structured mesh was composed of one million elements. Physical properties for bioreactor media were used with density $=1.01 \mathrm{~g} \mathrm{~cm}^{3}$ and dynamic Viscosity $=1.27$ centipoise. At the inlet, a physiologically valid velocity waveform (Lotz et al., 2002) was imposed (Figure 38). The outlet was set to 0 relative pressure and all walls were set to no-slip conditions. Simulations were run in a Hewlett Packard $^{\mathrm{TM}}$ work station with intel(R) Xeon(R) CPU, x5550@ $2.67 \mathrm{GHz}$ (2processors) with 16.0 GB installed memory and 64-bit windows 7 operating system. Independence tests were performed on mesh, time step, and 
periodicity for steady and pulsatile simulations accordingly. We monitored the area averaged wall shear stress on the surface, and a $5 \%$ error acceptance criterion in comparison with highly resolved simulations was used. All simulations converged to $10^{-9}$. We computed oscillations in the flow by using the OSI parameter described by $\mathrm{He}$ and $\mathrm{Ku}(\mathrm{He}$ and $\mathrm{Ku}, 1996)$ and the OSI-| $\overrightarrow{\tau \mid}$ parameter as described by (Salinas and Ramaswamy, 2014).

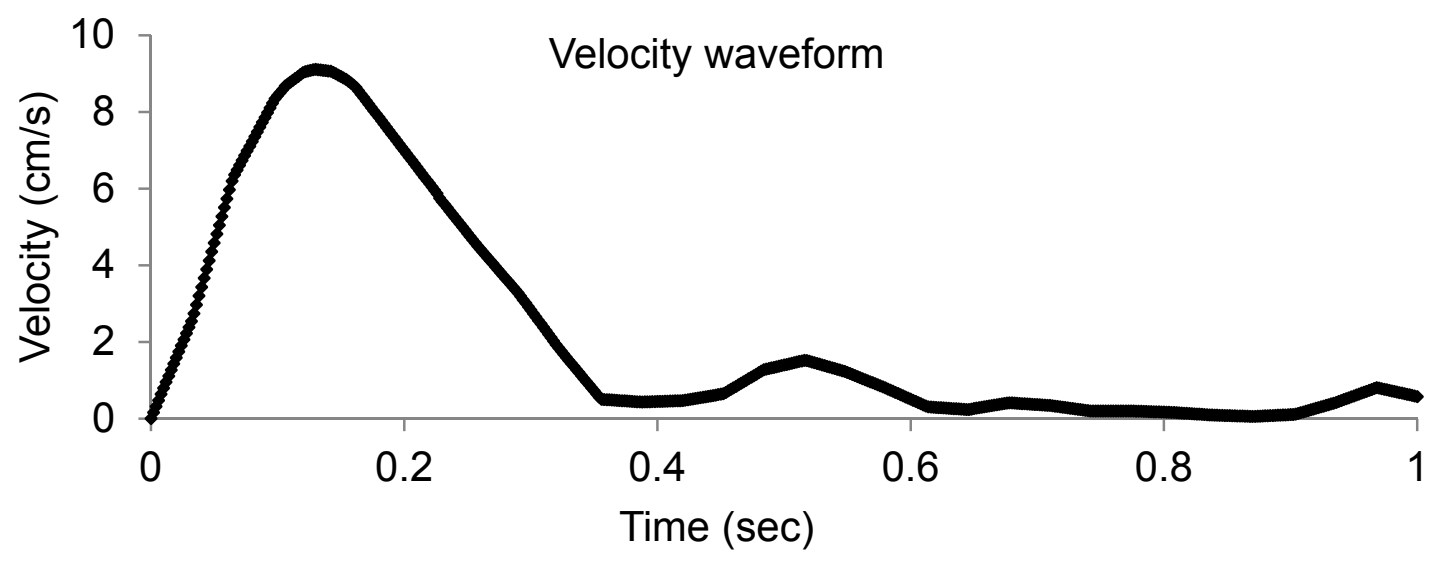

Figure 38. Velocity waveform with a velocity average of $0.199386 \mathrm{~cm} / \mathrm{s}$. It was used as the inlet velocity for the transient pulsatile simulations at 2 millisecond intervals.

\subsubsection{Statistics}

We performed analysis of variance tests in order to determine if the significant differences between control, steady flow and pulsatile flow groups. We used Tukey's test with a significance of $p=0.05$. Normality distributions and equal variance were assumed. 


\subsection{Results}

\subsubsection{In vitro Experiments}

In Figures 39-56 it we showed a section of the channel where the cells are attached to the walls. During cell seeding, the shape of the cells was mainly rounded and began to elongate in the following days. In the case of the steady flow, cells showed a slight formation in the flow axial direction. This event was also seen in the pulsatile flow group, but with less intensity than the steady flow case. Both steady and pulsatile groups showed higher number of cells attached to the walls when compared to the control group. Cells counts for each group were conducted and reported in Figure 57. Indeed, we observed that there were more cells in the pulsatile flow group followed by the steady flow case, and the control group, respectively. We performed an anova study which determined that there are significant differences between the means of these groups as shown in Table 10. At a significance of $p=0.05$, Tukey's test revealed that there are no significant differences between the three groups when compared to each other seen in table 11.
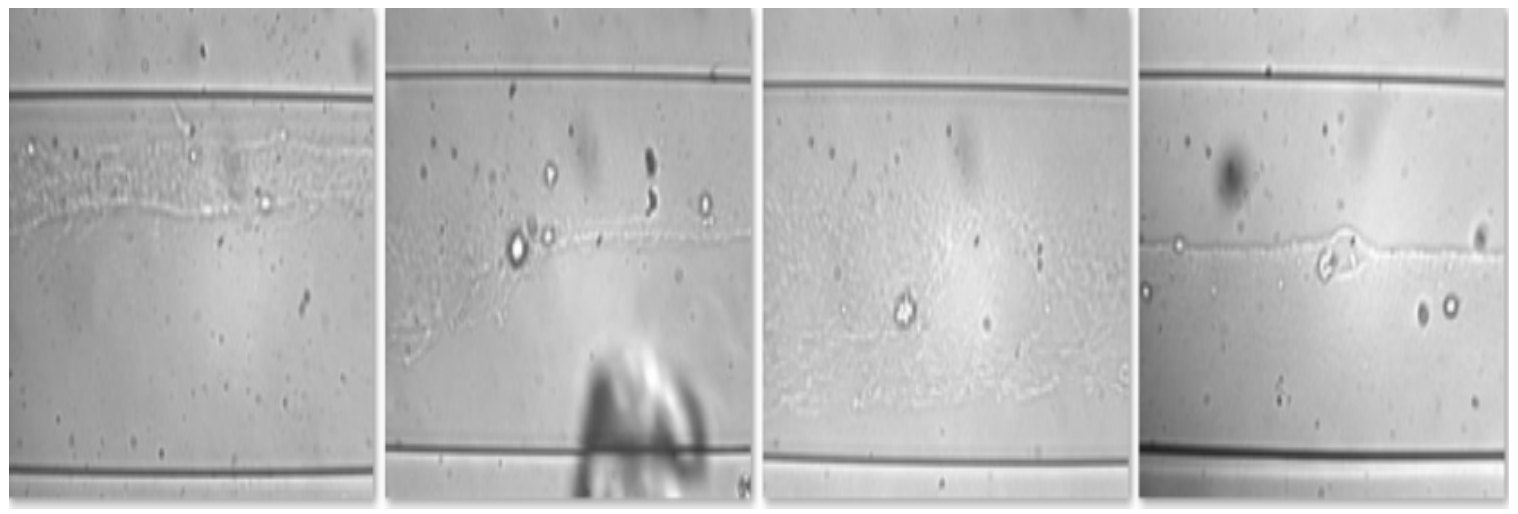

Figure 39. Control group. Cell attached to the walls of the bioflux 200 system day 1. 

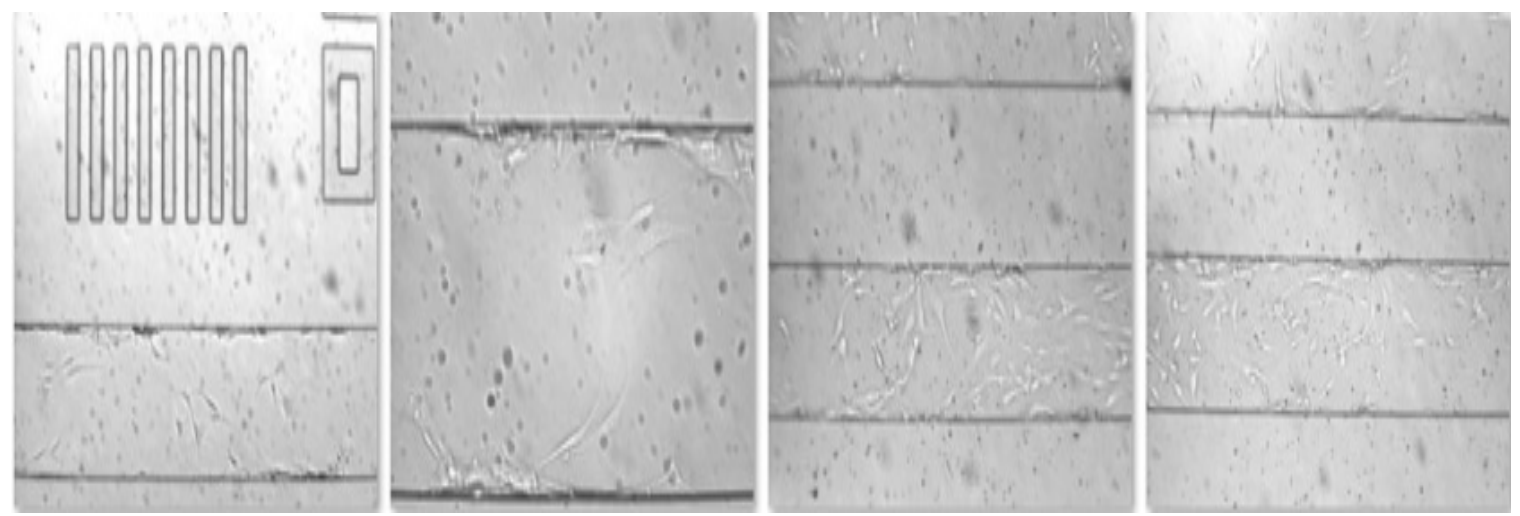

Figure 40. Control group. Cell attached to the walls of the bioflux 200 system day 5.
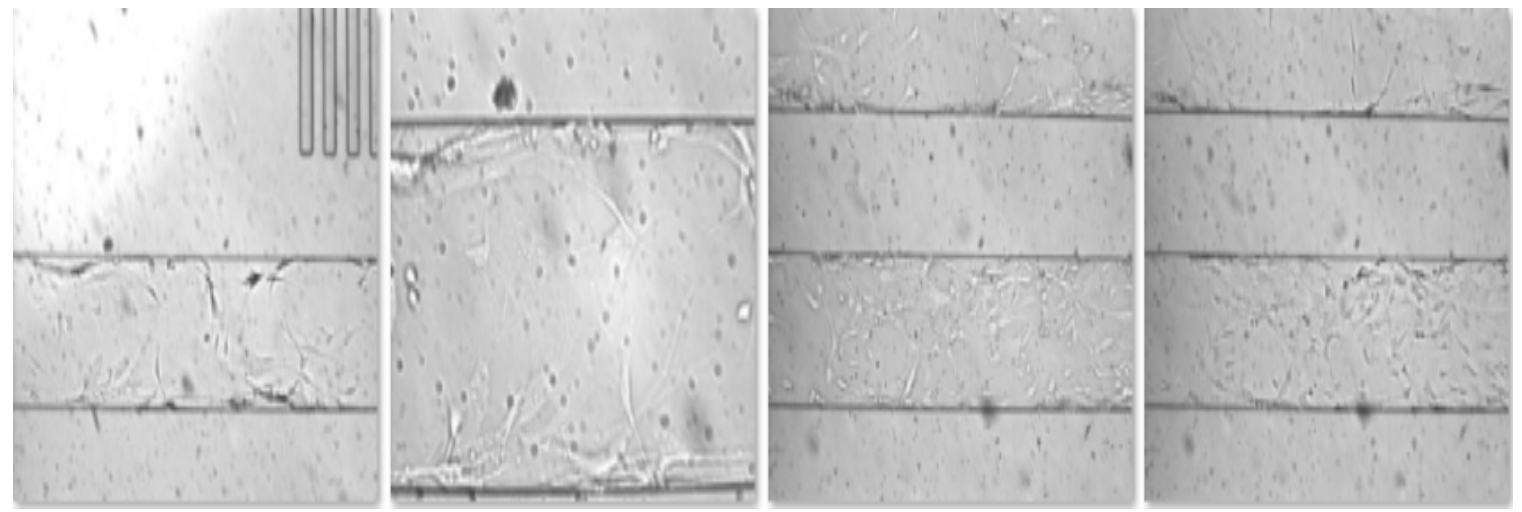

Figure 41. Control group. Cell attached to the walls of the bioflux 200 system day 6.
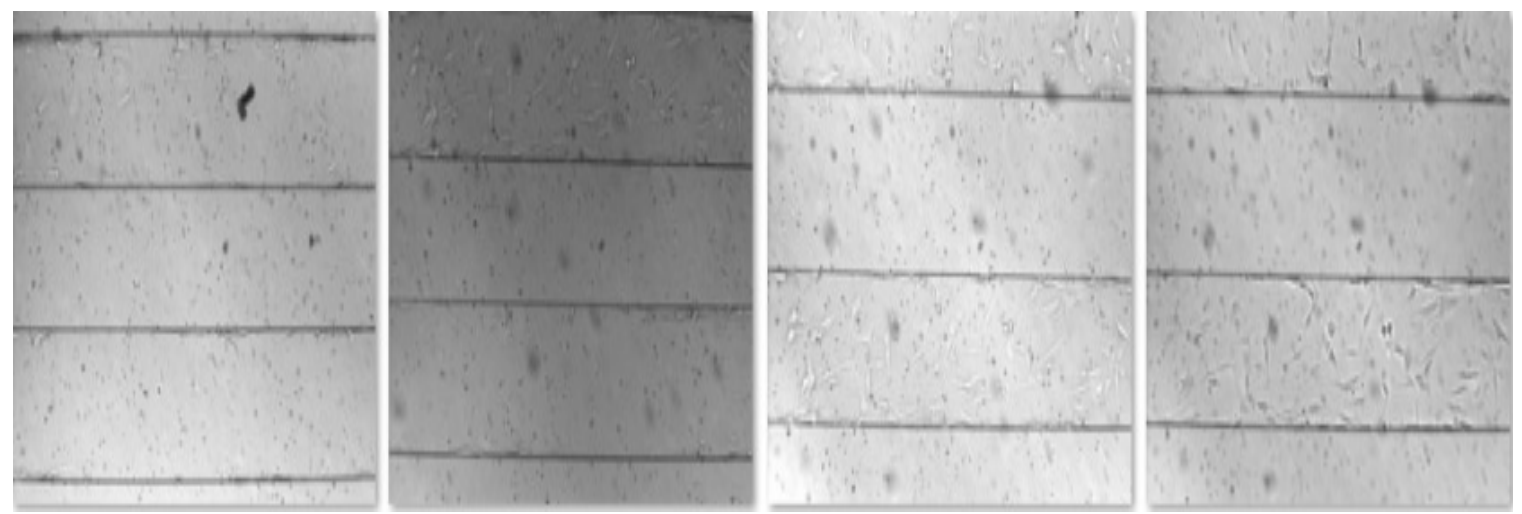

Figure 42. Control group. Cell attached to the walls of the bioflux 200 system day 7. 

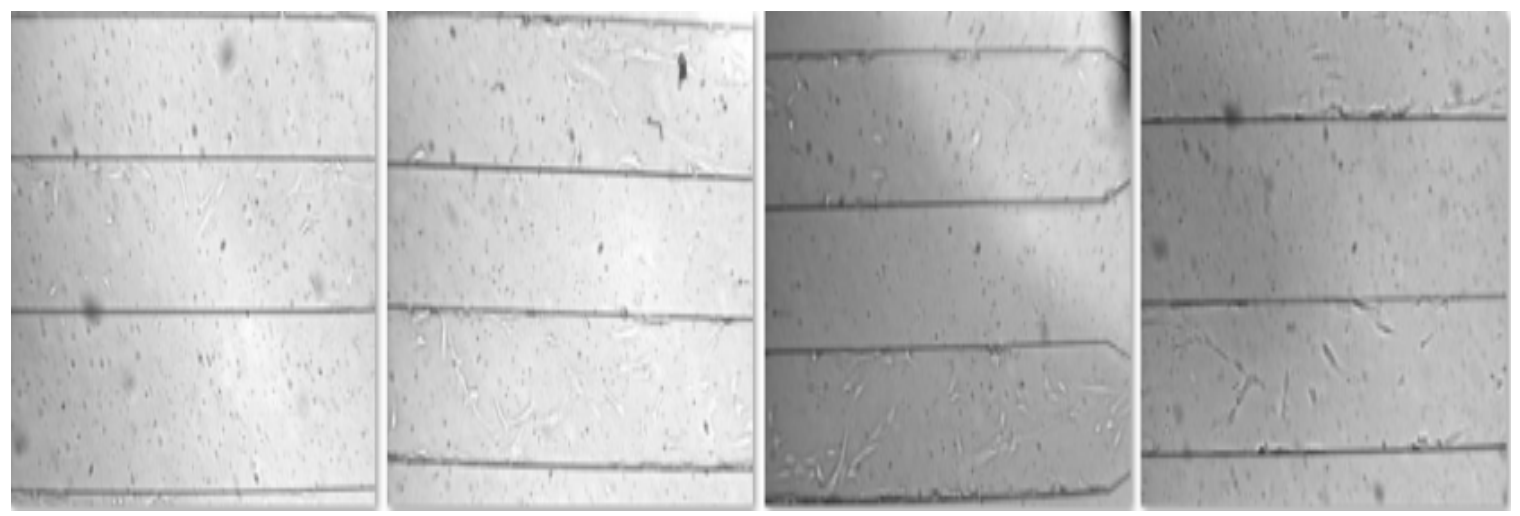

Figure 43. Steady flow group. Cell attached to the walls of the bioflux 200 system day 1.
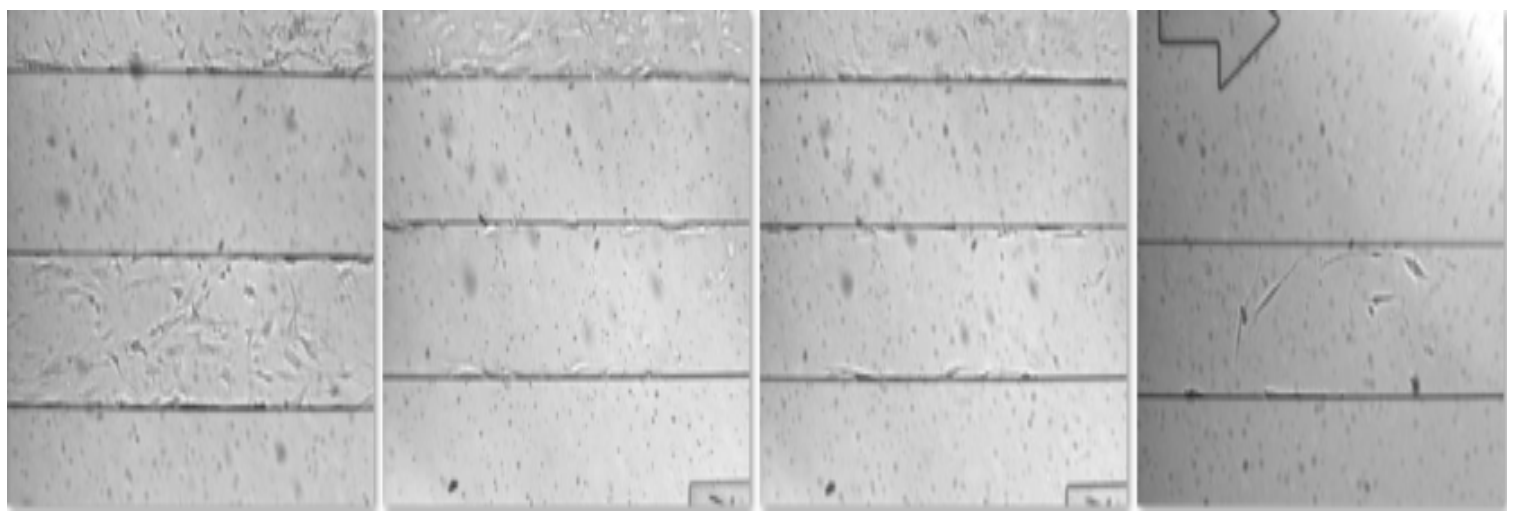

Figure 44. Steady flow group. Cell attached to the walls of the bioflux 200 system day 2.
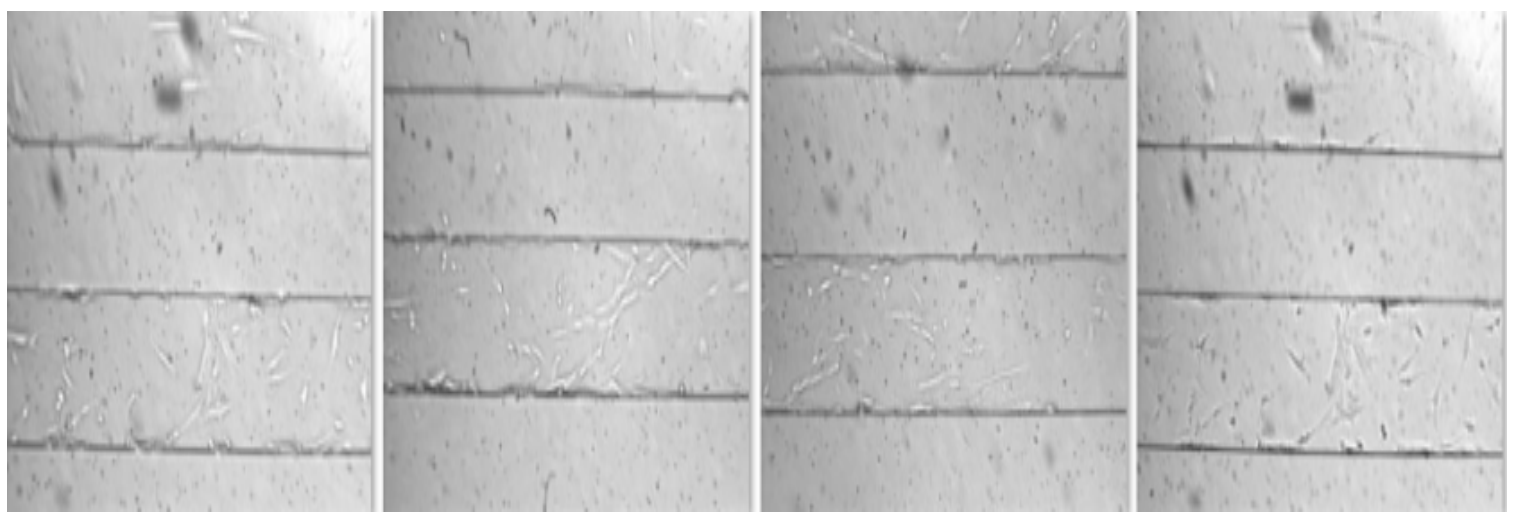

Figure 45. Steady flow group. Cell attached to the walls of the bioflux 200 system day 3. 

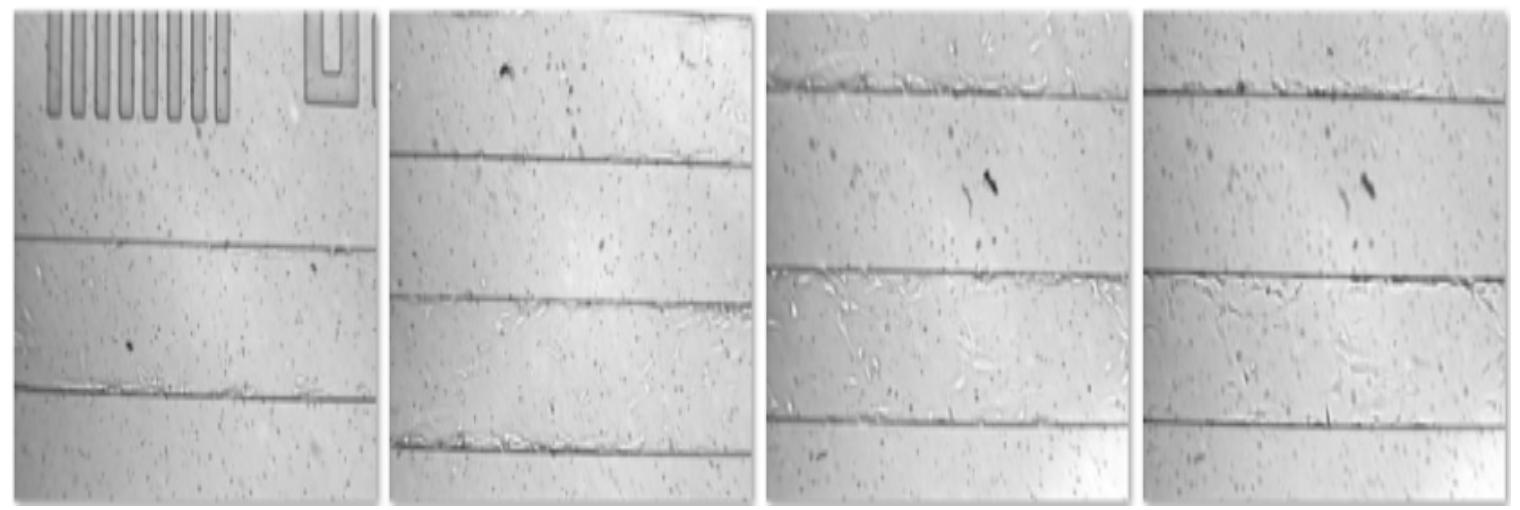

Figure 46. Steady flow group. Cell attached to the walls of the bioflux 200 system day 4 .
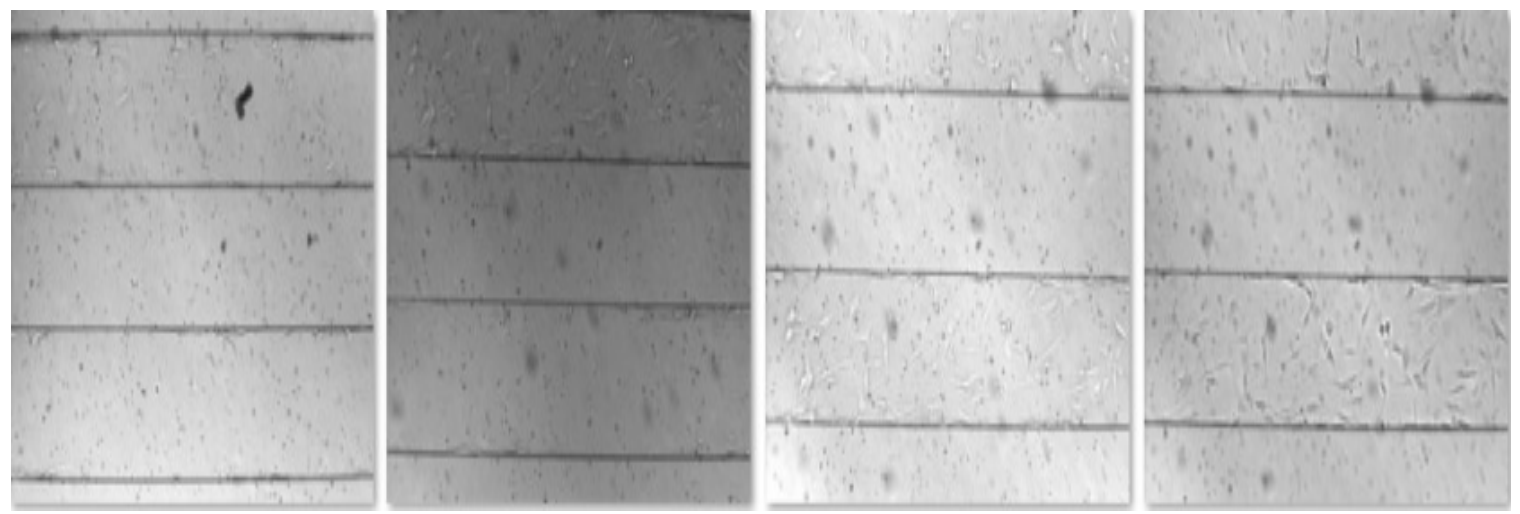

Figure 47. Steady flow group. Cell attached to the walls of the bioflux 200 system day 5 .
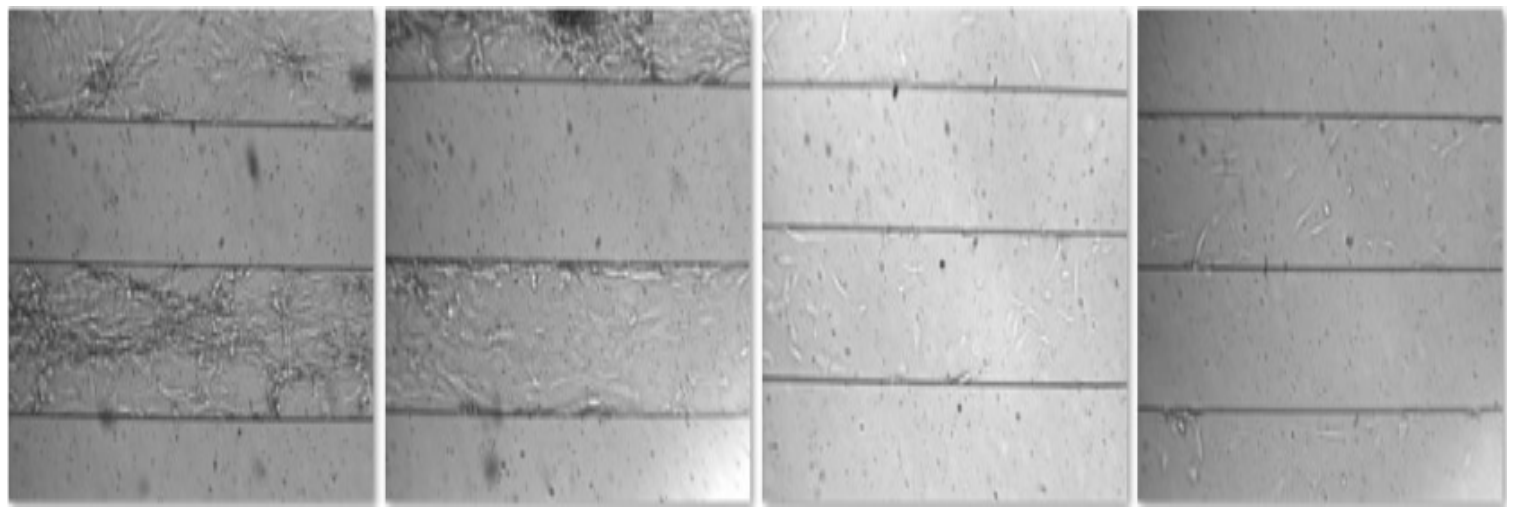

Figure 48 Steady flow group. Cell attached to the walls of the bioflux 200 system day 6. 

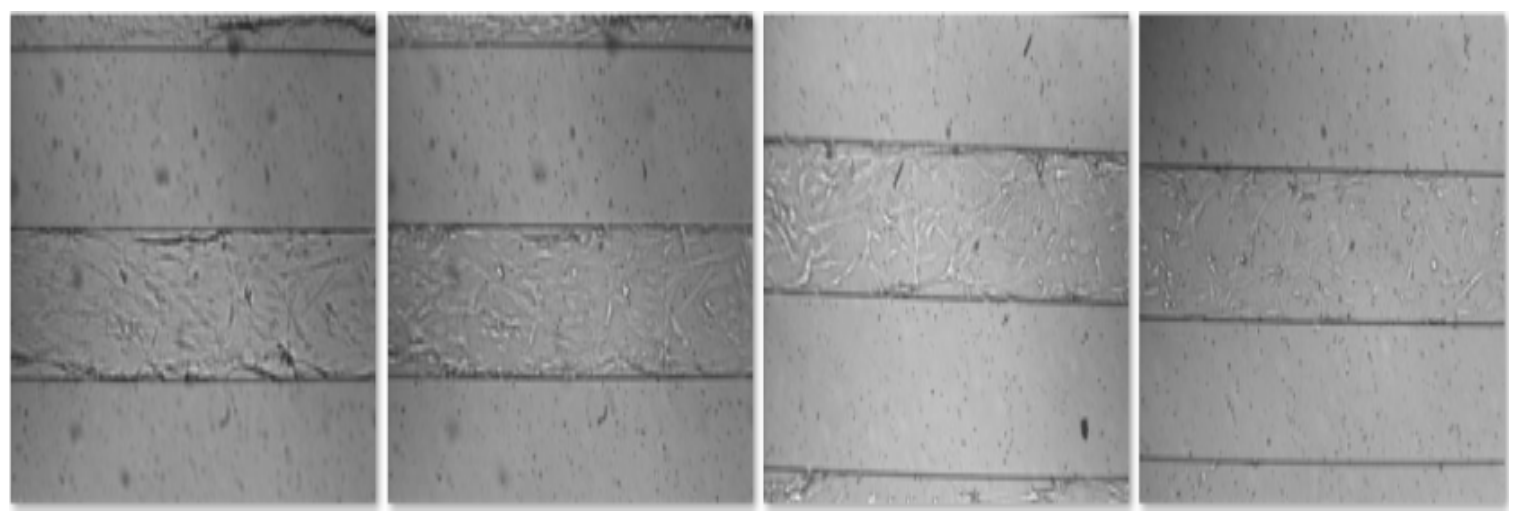

Figure 49. Steady flow group. Cell attached to the walls of the bioflux 200 system day 7 .
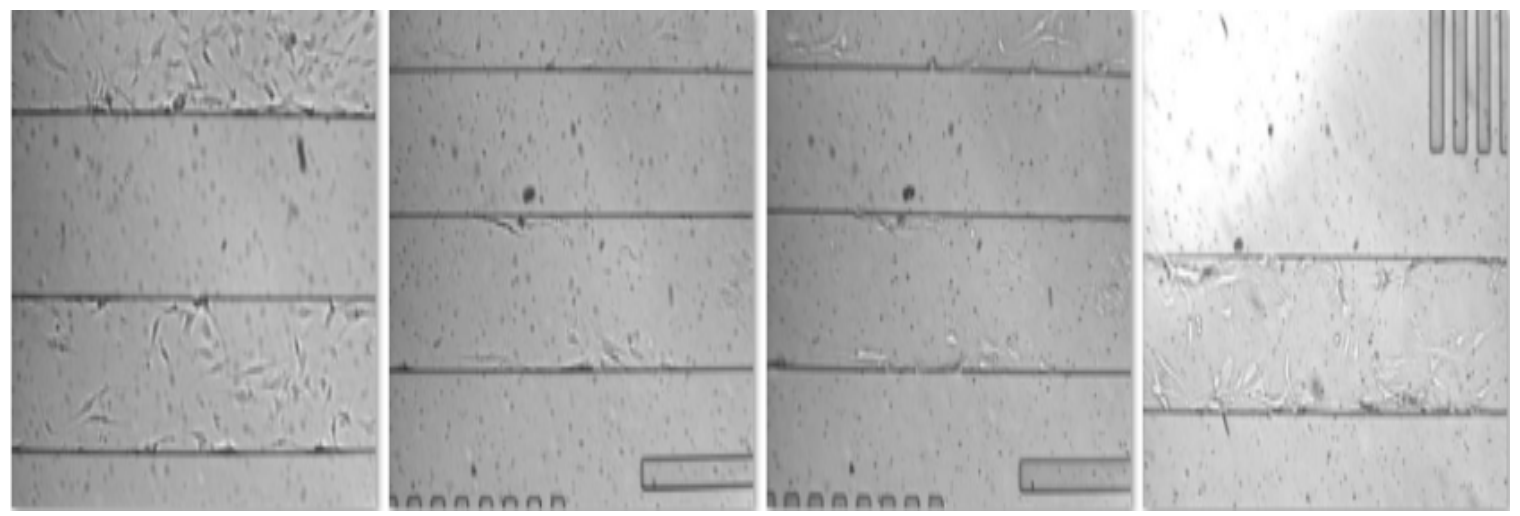

Figure 50. Pulsatile flow group. Cell attached to the walls of the bioflux 200 system day 1.
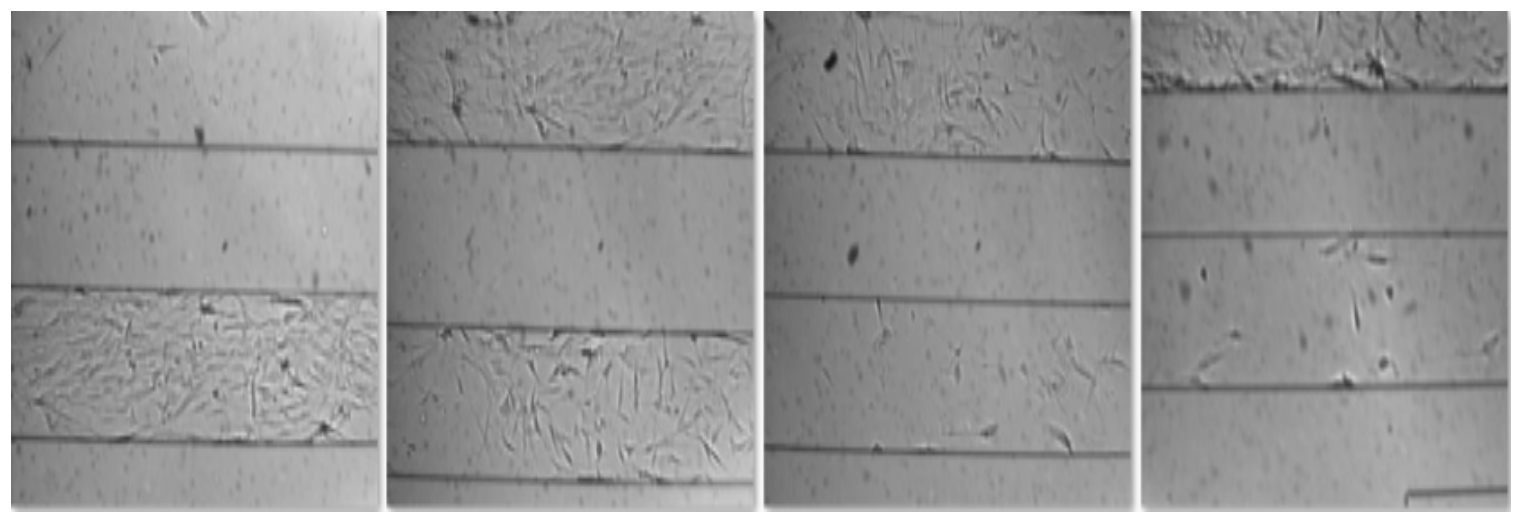

Figure 51. Pulsatile flow group. Cell attached to the walls of the bioflux 200 system day 2. 

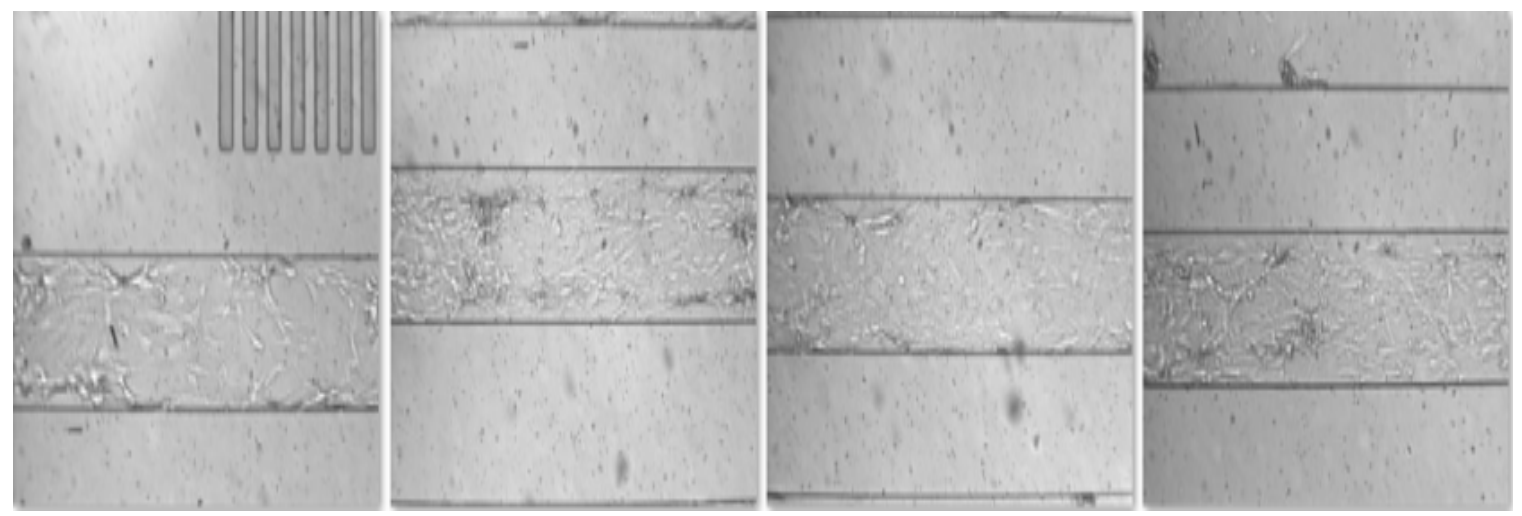

Figure 52. Pulsatile flow group. Cell attached to the walls of the bioflux 200 system day 3 .
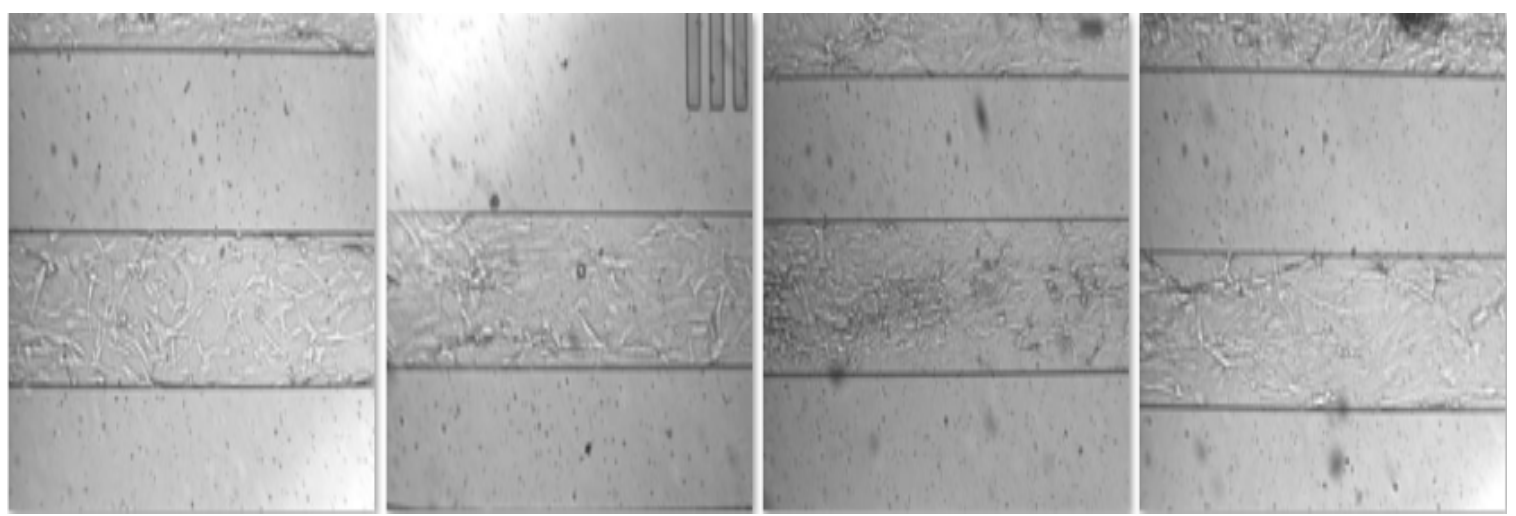

Figure 53. Pulsatile flow group. Cell attached to the walls of the bioflux 200 system day 4.
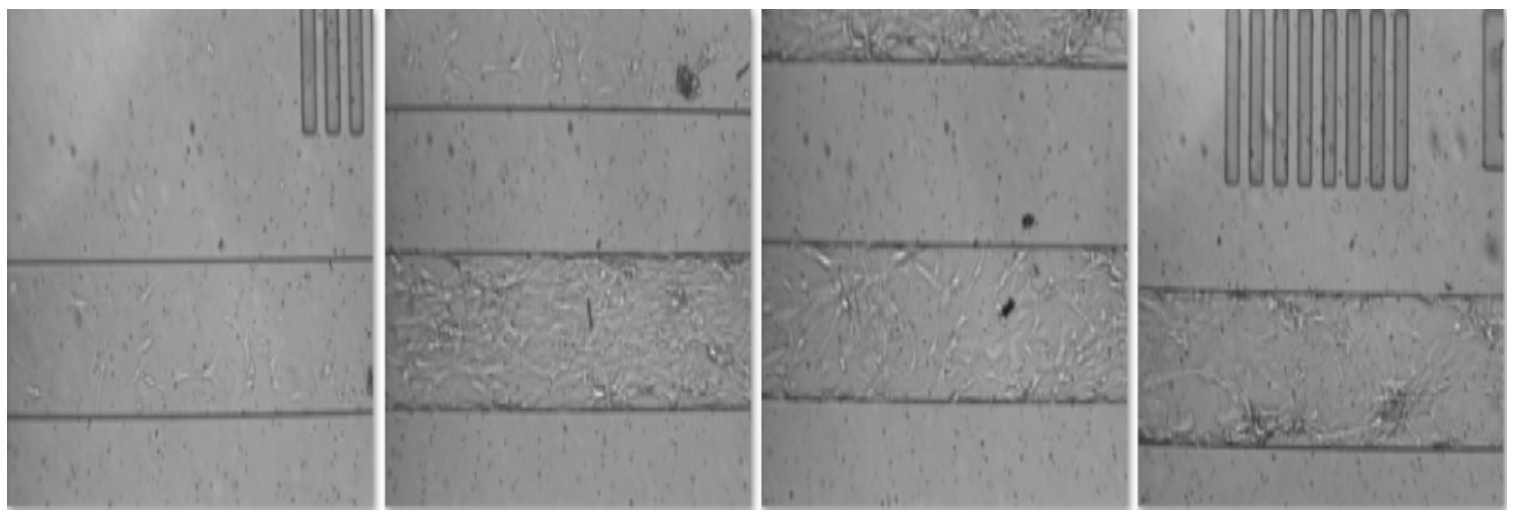

Figure 54. Pulsatile flow group. Cell attached to the walls of the bioflux 200 system day 5 . 

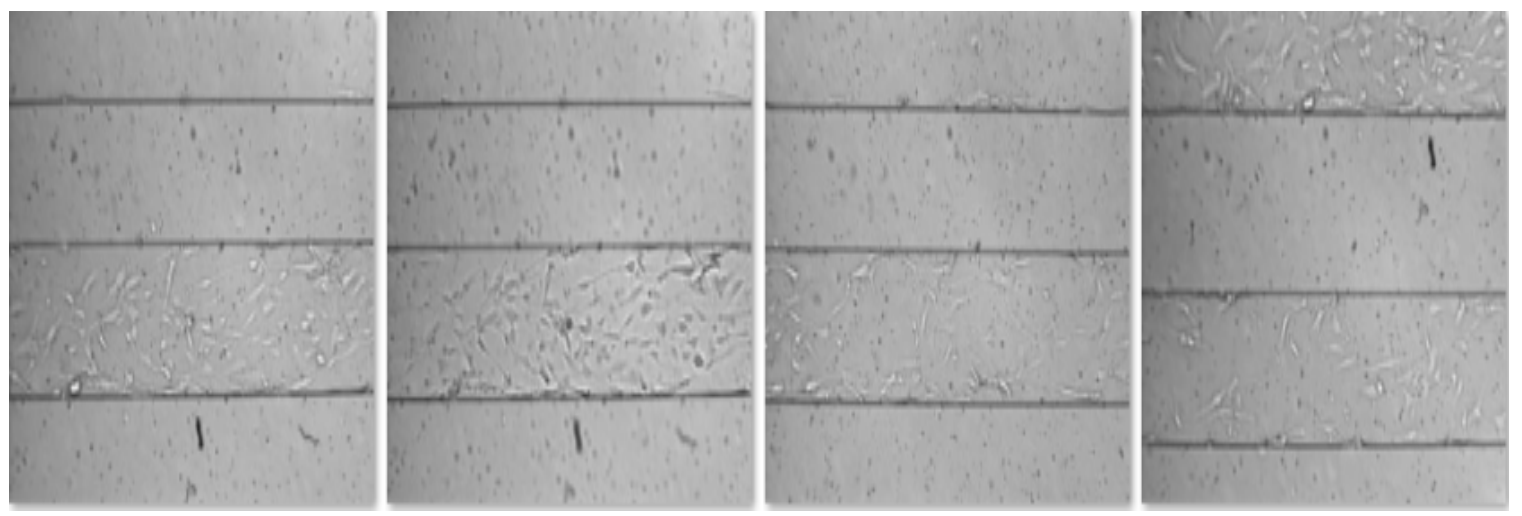

Figure 55. Pulsatile flow group. Cell attached to the walls of the bioflux 200 system day 6 .
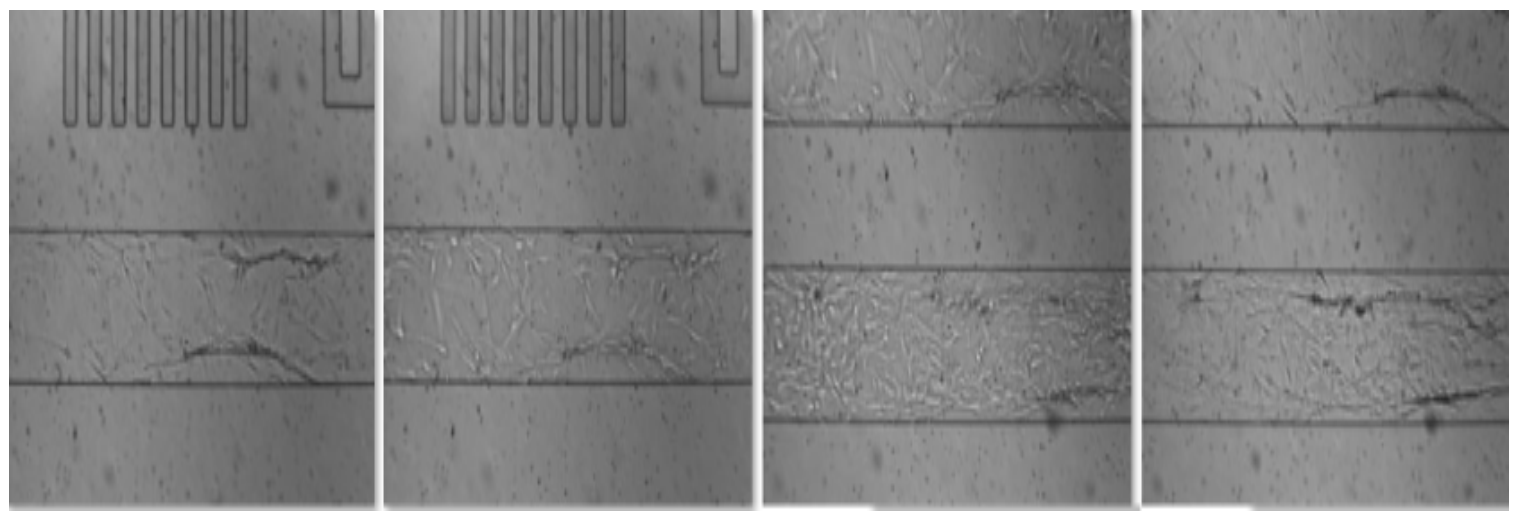

Figure 56. Pulsatile flow group. Cell attached to the walls of the bioflux 200 system day 7 .

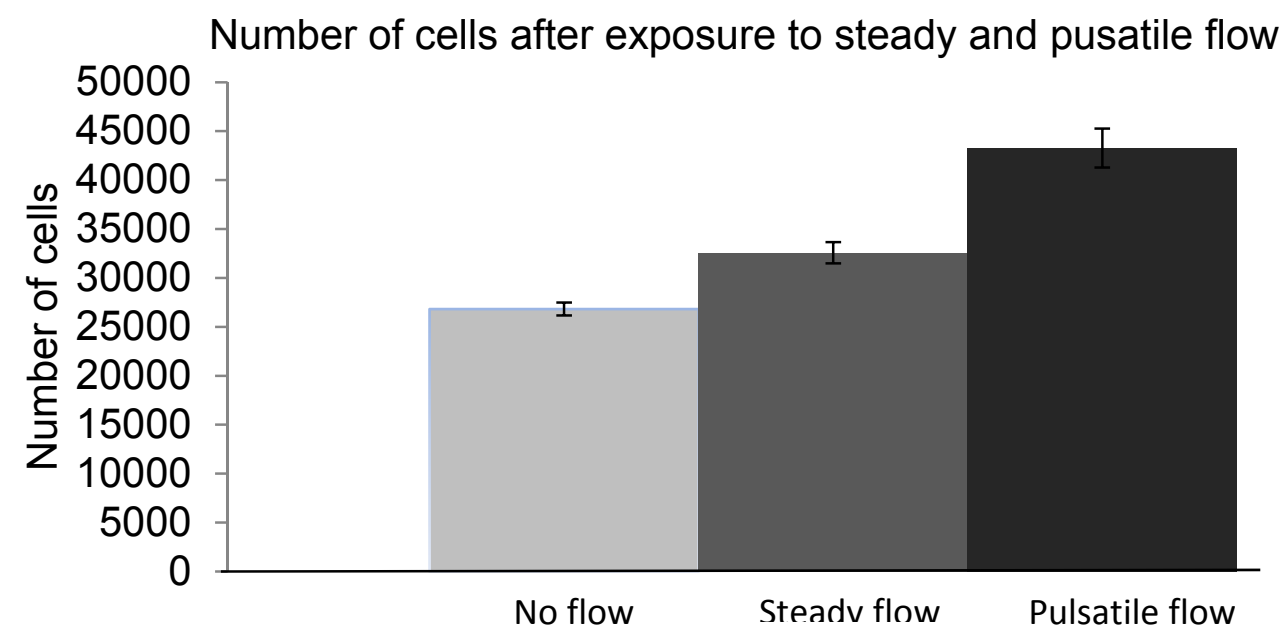

Figure 57. Bar chart showing the number of cells during control, steady, and pusatile flow experiments. 
ANOVA

Cell Count

\begin{tabular}{|l|r|r|r|r|r|}
\hline & Sum of Squares & df & Mean Square & F & Sig. \\
\hline Between Groups & 656472616.667 & 2 & 328236308.333 & 44.188 & .000 \\
Within Groups & 66853725.000 & 9 & 7428191.667 & & \\
Total & 723326341.667 & 11 & & & \\
\hline
\end{tabular}

Table 10. Anova results for cell count from control, steady and pulsatile flow conditions

Dependent Variable: Cell Count

Multiple Comparisons

Tukey HSD

\begin{tabular}{|c|c|c|c|c|c|c|}
\hline \multirow[t]{2}{*}{ (I) Flow } & \multirow[t]{2}{*}{ (J) Flow } & \multirow{2}{*}{$\begin{array}{c}\text { Mean Difference } \\
(\mathrm{I}-\mathrm{J})\end{array}$} & \multirow[t]{2}{*}{ Std. Error } & \multirow[t]{2}{*}{ Sig. } & \multicolumn{2}{|c|}{ 95\% Confidence Interval } \\
\hline & & & & & Lower Bound & $\begin{array}{l}\text { Upper } \\
\text { Bound }\end{array}$ \\
\hline \multirow{2}{*}{ No flow } & Steady flow & -5457.50000 & 1927.19896 & .047 & -10838.2503 & -76.7497 \\
\hline & Pulsatile flow & $-17690.00000^{x}$ & 1927.19896 & .000 & -23070.7503 & -12309.2497 \\
\hline \multirow{2}{*}{ Steady flow } & No flow & 5457.50000 & 1927.19896 & .047 & 76.7497 & 10838.2503 \\
\hline & Pulsatile flow & -12232.50000 & $\begin{array}{l}1927.19896 \\
192719896\end{array}$ & $\begin{array}{l}.000 \\
000\end{array}$ & $\begin{array}{r}-17613.2503 \\
123092497\end{array}$ & $\begin{array}{r}-6851.7497 \\
230707503\end{array}$ \\
\hline Pulsatile flow & Steady flow & $12232.50000^{*}$ & & .000 & & 17613.2503 \\
\hline
\end{tabular}

Table 11. Post hoc tests. Multiple comparisons using Tukey's test for cell counts from control, steady and pulsatile flow conditions.

Figures 58-60 indicate gene expression of BMSCs when exposed to

control, steady flow and pulsatile flow environments, respectively. The graph was normalized to GAPDH. In the control group there was strong expression of GAPDH and B-integrin genes. Also, there was weak expression of PECAN-1 and VE-Cadherin genes. In the steady flow group there was strong expression of GAPDH and B-integrin PECAN-1 and VE-Cadherin genes. In the pulsatile flow group there was strong expression of GAPDH and B-integrin, Thy-1, and Tie-1 genes. Also, there was weak expression of PECAN-1, VE-Cadherin, and FLK-1 genes.

Previously computed OSI contours (Rath et al., 2014) are shown in figure 61a. In can be seen that there was high oscillatory shear environments acting on the walls of the channels seeded with BMSCs. The area averaged OSI was 0.49 . 
In this study we also reported on the TSSM that the walls were exposed to, and it is shown in Figure 61b. The area averaged TSSM was $197 \mathrm{~Pa}$. Finally, we computed the OSI-| $\overrightarrow{|\tau|}$, shown in Figure 61c. The area averaged OSI-| $\overrightarrow{\tau \mid}$ was 195 Pa. Due to the high OSI values, the OSI- $\overrightarrow{|\tau|}$ values approximate to the time averaged shear stresses per our definition of OSI- $\overrightarrow{|\tau|}$ (Salinas and Ramaswamy, 2014), which was our original intent when defining it in this manner.

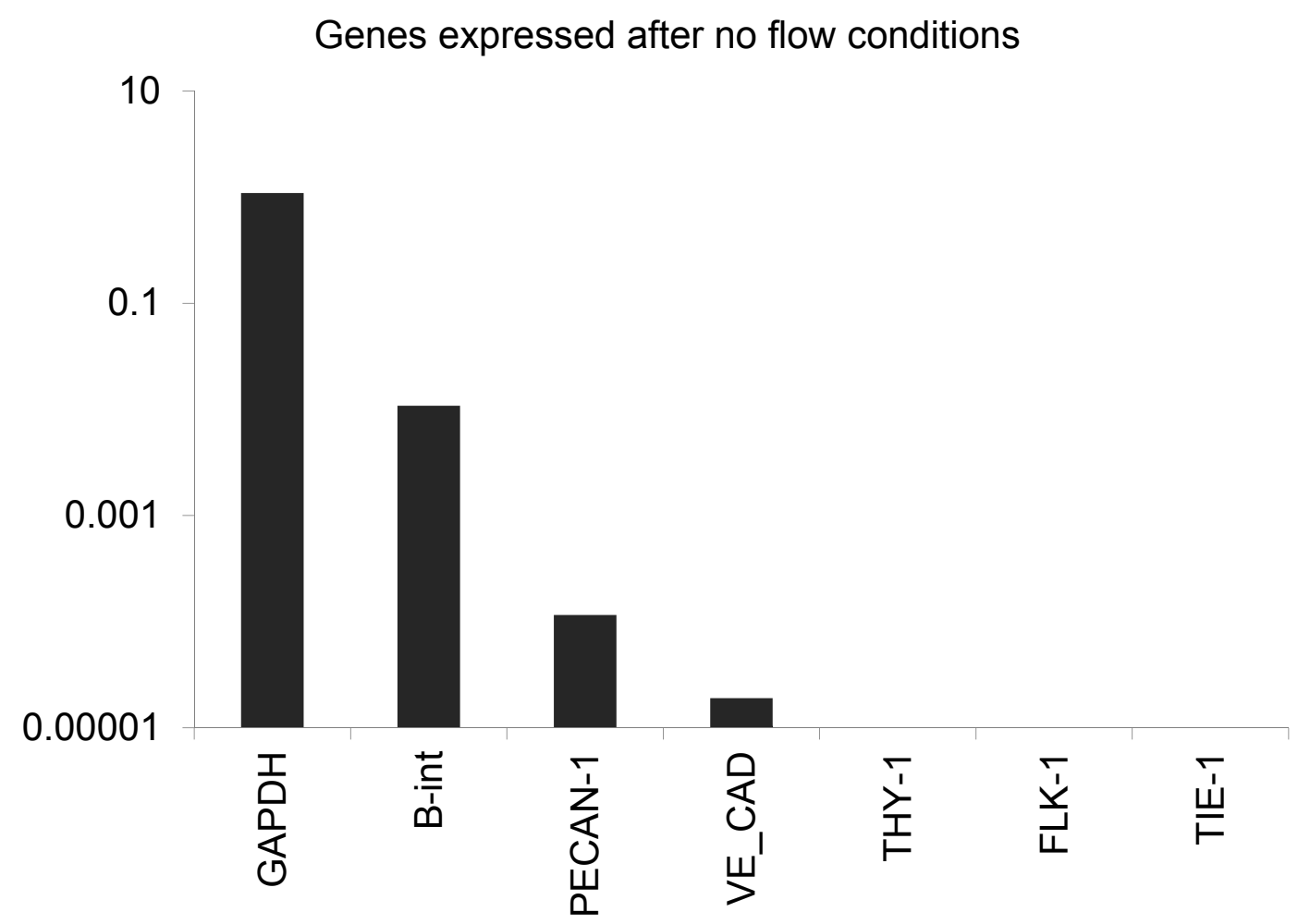

Figure 58. Genes expressed after control conditions. 


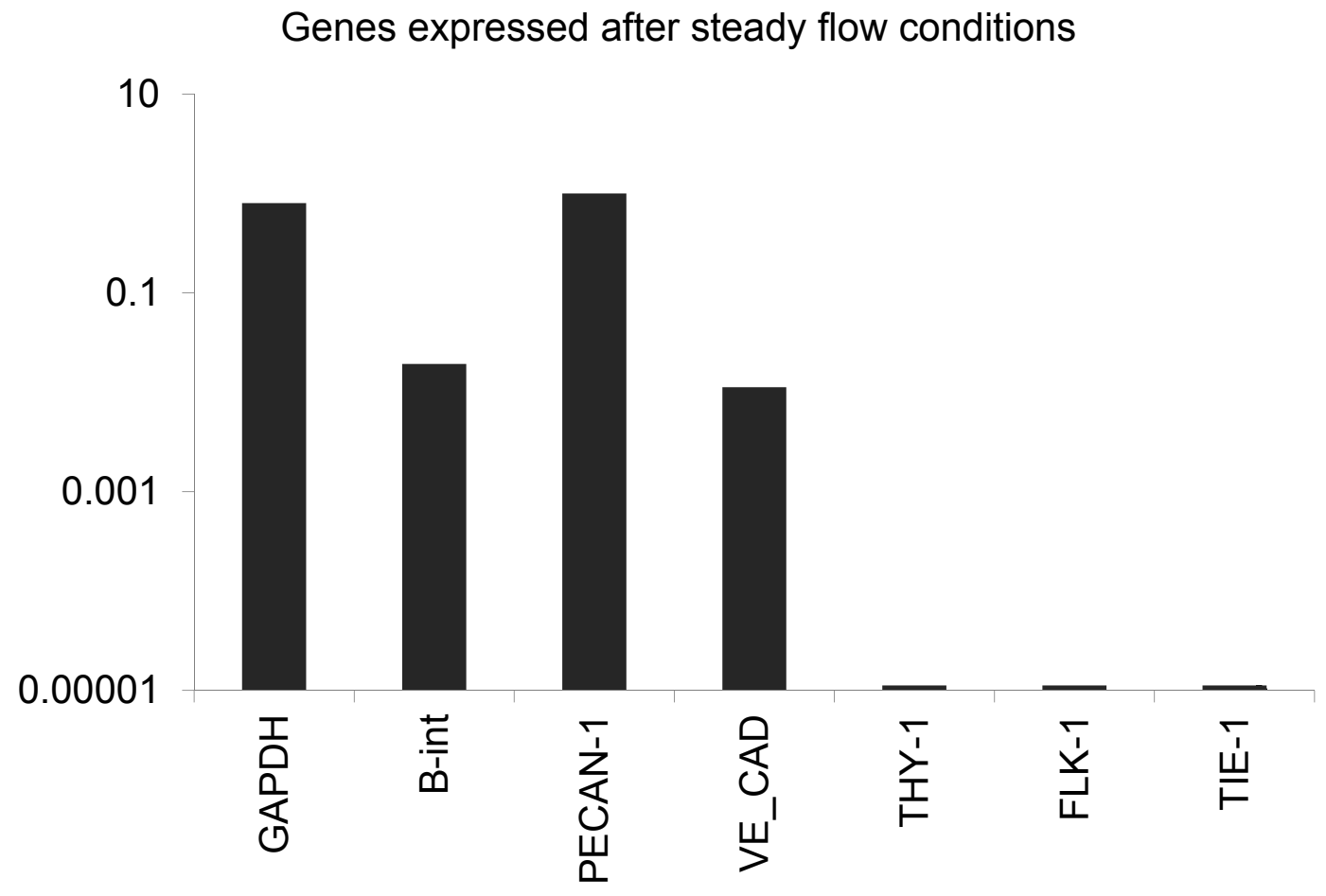

Figure 59. Genes expressed after steady flow conditions.

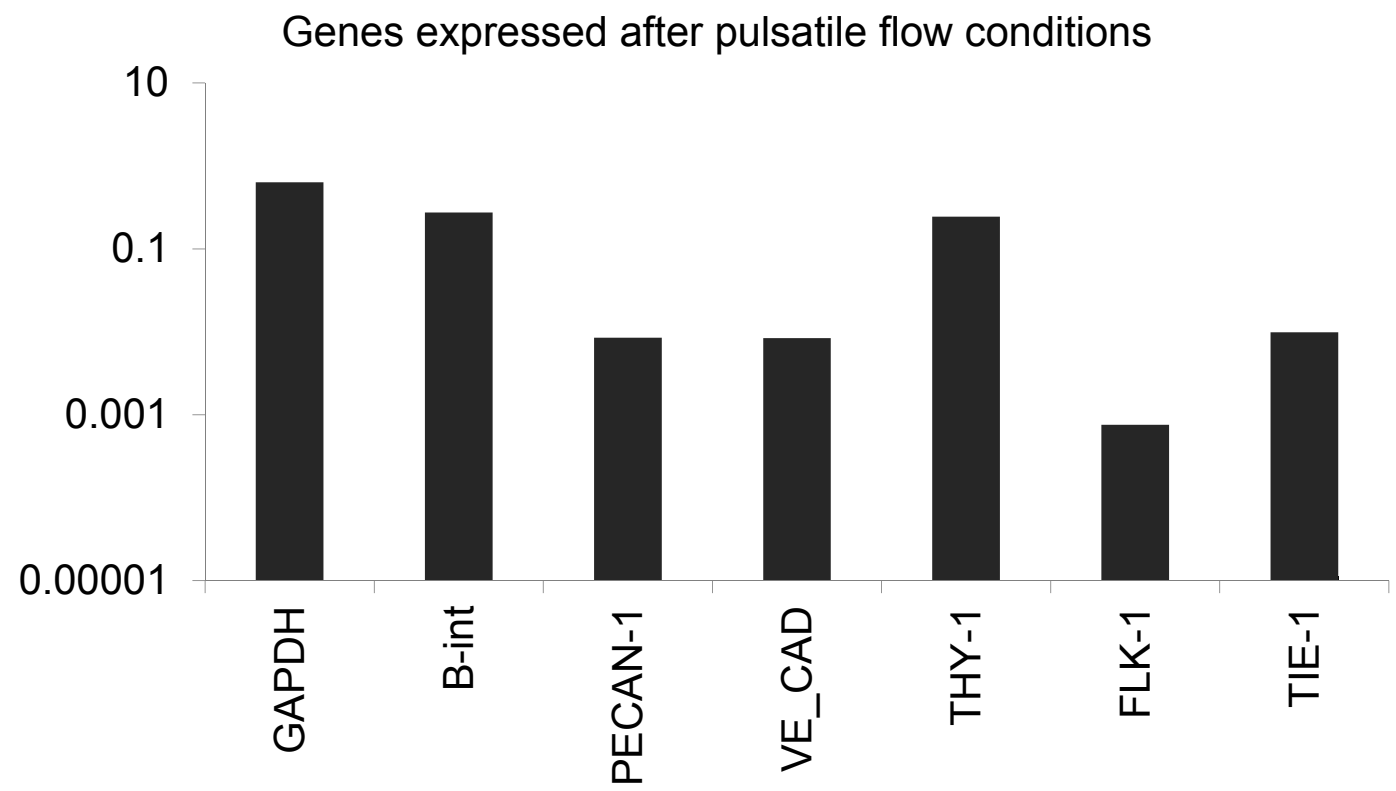

Figure 60. Genes expressed after pulsatile flow conditions. 
a)

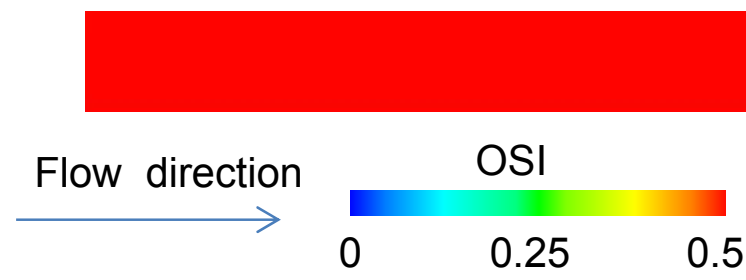

b)

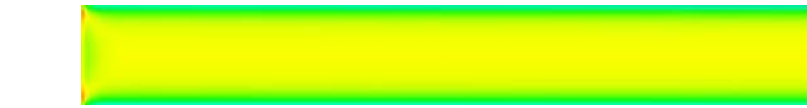

Flow direction Time averaged shear stress $(\mathrm{Pa})$

$60 \quad 170 \quad 280$

c)
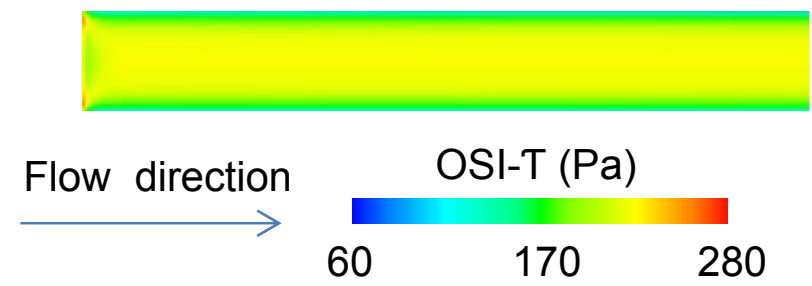

Figure 61. Contours of a) OSI (Rath et al., 2014) , b) Time averaged shear stress, and c) OSI- $\overrightarrow{|\boldsymbol{\tau}|}$ of the bioflux 200 rectangular channel seeded with BMSCs.

\subsection{Discussion}

OSI- $\overrightarrow{|\tau|}$ computationally obtained by (Salinas and Ramaswamy, 2014) has been linked to promote engineered collagen formation by BMSCs in vitro heart valve tissue engineering experiments (Engelmayr et al., 2006). However, OSI-| $\overrightarrow{|\tau|}$ has not been associated to increase BMSCs proliferation or gene expression. In this study we decided to conduct a preliminary in vitro experiment where BMSCs where exposed to cell culture environments where OSI-| $\overrightarrow{\tau \mid}$ can be maximized within physiologically valid ranges. Previously, we reported that oscillatory shear environments can be maximized by using a physiologically derived pulsatile flow 
source acing on straight static specimens (Salinas et al., 2014a; Salinas et al., 2014b). We noted that this set up should also have high OSI-| $\overrightarrow{\tau \mid}$ values, which encouraged us to seed BMSCs in perfectly flat rectangular cell culture channels in a bioflux200 system (Fluxion Biosciences, San Francisco, CA) and expose them to pulsatile flow.

We computationally simulated the experimental set up in Ansys CFX (Ansys, Canonsburg, PA) and found that indeed OSI- $\overrightarrow{|\tau|}$ strongly acted on the surfaces of the BMSCs seeded channels as seen in Figure 60c. Given the high degree of oscillations in the wall shear direction (Figure 61a), the OSI- $\overrightarrow{|\tau|}$ closely resembled the TSSM values (Figure 61b). We note that in the control and steady flow cases, there was no presence of OSI-| $\overrightarrow{\tau \mid}$ values given that they are not time dependent scenarios. OSI- $\overrightarrow{|\tau|}$ was only present in the pulsatile case. There was significantly $(p<0.05)$ higher number of cells in the pulsatile case when compared to the control and steady flow cases (Table 11). In addition, there was significant $(p>0.05)$ differences between the control and steady group. This finding suggests that while shear stress along does mildly influence cell proliferation when acting alone, when accompanied by oscillatory shear environments there is enhanced BMSCs proliferation.

We considered genetic markers for smooth muscle cells (SMCs) particularly integrin, beta1, THY-1, FZD2, and MLC1F. We also studied endothelial cell (ECs) genes comprising PECAN-1, FLK1, and VE-cadherin. Gene expression assays revealed that all groups had strong presence of GAPDH 
B-int, PECAN1, and VE-cadherin genes with the exception that the control group showed weak presence of Pecan-1 and VE-cadherin as seen in Figure 58-60. In addition, the pulsatile case group showed strong presence of Thy-1 and Tie-1 genes and significant presence of Flk-1 gene when compared to control and steady groups which did not account for these genes. In brief, B-int (integrin beta1) is involved in cell adhesion, tissue repair, and embryogenesis; THY-1 regulates adhesion, migration, and fibrosis cell- cell, and cell-matrix interactions. PECAM1 participates in leukocyte migration, angiogenesis, and integrin activation. FLK1 is a signaling protein for vascular endothelial growth factor; TIE1 regulates cell adhesion molecules. Lastly, VE-cadherin participates in angiogenesis (Martinez et al., 2013).

Importantly, Thy-1 has been shown to be present in undifferentiated stem cells (Martinez et al., 2013). Thus as a preliminary experimental finding, we have found here through increased Thy-1 expression from oscillatory flow conditions, that BMSCs remain in a less differentiated state. This has important implications from a heart valve tissue engineering stand point as a TEHV could benefit from the plasticity of cell lineages during in vitro studies using heart valve like geometries or after in vivo implantation. Further studies to confirm BMSC-based gene expression outcomes under different flow states are ongoing by other investigators in our laboratories. 


\section{CHAPTER 7: CONCLUSIONS, LIMITATIONS, FUTURE WORK}

In this study we developed moving boundary CFD simulations to study the effects of movement of engineered tissue constructs on the flow physics and mass transport. In addition, we correlated the computational results with engineered collagen formation, cell proliferation and cell phenotype from literature and in-house in vitro experiments. We presented a modification of the traditional OSI parameter (He and Ku, J. Biomech Eng., 1996) given by: OSI-| $\overrightarrow{\tau \mid}$ $=2{ }^{*} \mathrm{OSI}{ }^{*} \int_{0}^{T} a b s(\tau) d t$ and demonstrated that the OSI- $\overrightarrow{|\tau|}$ correlated strongly with the greatly augmented engineered collagen production reported from previous in vitro experiments (Engelmayr et al., 2006), under flex-flow conditioning regimens.

We presented a convective-diffusive mass transport model to study sample movement effects on mass transport of glucose and oxygen. We found that sample flexure of $75 \%$ porous scaffolds did not enhance mass transport of glucose and oxygen, but sample flexure of $5 \%$ porous scaffolds did enhance mass transport of glucose and oxygen. We noted that in tissue engineered studies, sample porosity ranges from $92-0.73 \%$ porosity (Yu et al. Int J. Heat \&Mass transfer, 2009). This suggests that mass transport did not enhance tissue production reported in (Engelmayr et al., 2006). We reported that mass transport of glucose and oxygen is independent of specimen shape as seen by the flowalone cases with straight, semi-bent and fully bent sample geometries. We note that in this particular study, the initial definition of OSI-| $\overrightarrow{|\tau|}$ (Salinas and 
Ramaswamy, J. Biomech, 2014) was the only fluid induced parameter that correlated with augmented tissue formation by BMSCs. We computationally showed that OSI- $\mid \overrightarrow{\tau \mid}$ environments can be maximized during conditions of high oscillatory shear caused by physiologically valid pulsatile flow conditions (Figure 61). In an effort to observe to effect of OSI-| $\overrightarrow{|\tau|}$ on cell proliferation and cell phenotype, we conducted an in vitro experiment where BMSCs implanted on cell culture channels in a bioflux200 where exposed physiologically valid pulsatile conditions. We showed that OSI- $\overrightarrow{|\tau|}$ environments up-regulated cell differentiation and cell phenotype. We thus recommend that heart valve tissue engineering protocols utilize physiologically valid pulsatile flow sources to mechanically condition, by means of OSI- $\overrightarrow{|\tau|}$ environments, BMSCs implanted in rectangular cell/tissue culture environments as it has been shown to play a significant role in tissue formation, cell proliferation and cell phenotype.

In this study, our mass transport model considered only two species: glucose and oxygen. In addition, it only considered diffusivity effects in cell culture media and scaffold matrix. Furthermore we did not consider any stretch modes. Stretching of engineered tissue constructs may mediate BMSCs activity. Our in vitro study was conducted in a 2-D set up and did not account for collagen formation.

Future studies in our laboratories will be aimed at addressing the main limitations described above. The mass transport model will be modified in order to include cell specific permeability and account for more species including for 
example lactate and important growth factors. The 3-D in-house cell and tissue culture studies will be performed using an FSF bioreactor containing BMSCs seeded porous scaffolds fixed in a straight configuration. A physiologically valid pulsatile flow source will be used to mechanically condition the engineered constructs and the results of BMSC gene expression will be correlated to shear stress and shear stress variants (e.g. OSI and OSI-| $\overrightarrow{|\tau|}$ ).

As an initial effort, we have conducted the CFD simulations based on a square pulsatile velocity waveform (Figure 62) that yielded $850 \mathrm{ml} / \mathrm{min}$ flow rate used in similar bioreactor studies (Ramaswamy et al., 2014). We calculated the TSSM, OSI and OSI-| $\overrightarrow{\tau \mid}$ values for the three rectangular specimens, which are shown in Figure 63. The area averaged values for the inner wall $(n=3)$ were 0.15 $\pm 0.011 \mathrm{~Pa}, 0.45 \pm 0.023,0.14 \pm 0.015 \mathrm{~Pa}$ for TSSM, OSI and OSI-|च| respectively. In the case of the outer wall, the area averaged values were $0.12 \pm$ $0.012 \mathrm{~Pa}, 0.29 \pm 0.019,0.073 \pm 0.002 \mathrm{~Pa}$ for TSSM, OSI and OSI-| $\overrightarrow{\tau \mid}$ respectively. Clearly, these CFD results (Figure 63a) show that relatively large magnitudes of OSI- $\overrightarrow{|\tau|}$ can be created through flow pulsatility on fixed, nonmoving specimens. In other words, any effect by specimen movement to improve engineered tissue formation and gene expression outcomes from BMSCs, via altered mass transport mechanisms will be eliminated. Future bioreactor experiments in our laboratories, in-line with these CFD simulations will delineate if OSI- $\overrightarrow{|\tau|}$ is the sole regulator of enhanced BMSCs gene expression and tissue formation in the context of the heart valve phenotype. 


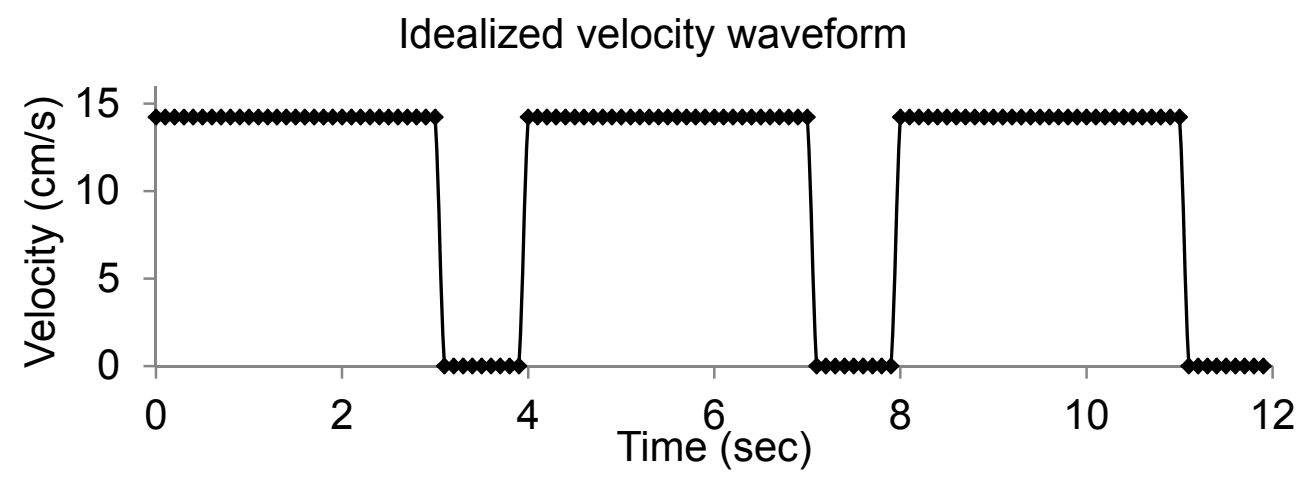

Figure 62. Idealized velocity waveform.

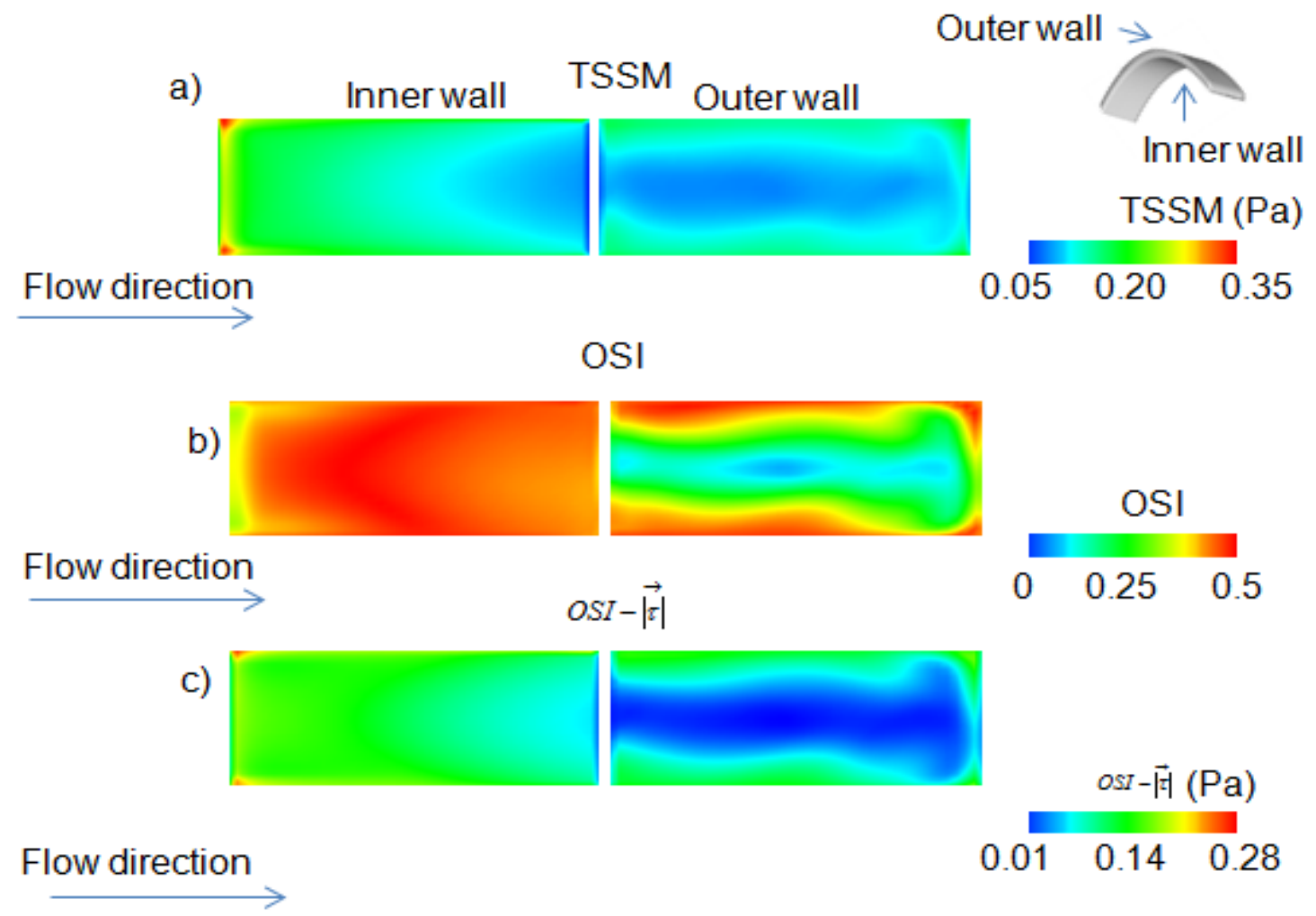

Figure 63. Contours of the inner and outer walls of the fixed rectangular specimens for a) TSSM; b) OSI; c) osi- $|\overrightarrow{\boldsymbol{\tau}}|$. 


\section{REFERENCES}

Abraham, J. P., Gorman, J. M., Sparrow, E. M., Stark, J. R., Kohler, R. E., 2013. A mass transfer model of temporal drug deposition in artery walls. International Journal of Heat and Mass Transfer 58, 632-638.

Ai, L., Vafai, K., 2006. A coupling model for macromolecule transport in a stenosed arterial wall. International Journal of Heat and Mass Transfer 49, 15681591.

Anastasiadis, K., Kambouroglou, D., Spanos, P., 2004. The use of Valve Homografts and Autografts in Adult Cardiac Surgery. Hellenic Journal of Cardiology, 36-41.

Ansys, 2014. Ansys cfx Solver theory guide. Release 15.0.

Arcidiacono, G., Corvi, A., Severi, T., 2005. Functional analysis of bioprosthetic heart valves. Journal of Biomechanics 38, 1483-1490.

Arnsdorf, E. J., Tummala, P., Jacobs, C. R., 2009a. Non-Canonical Wnt Signaling and N-Cadherin Related b-Catenin Signaling Play a Role in Mechanically Induced Osteogenic Cell Fate. PLos One 4, e5388.

Arnsdorf, E., Tummala, P., Kwon, R., Jacobs, C., 2009b. Mechanically induced osteogenic differentiation-the role of RhoA, ROCKII and cytoskeletal dynamics. Journal of Cell Science 122, 546-553.

Athanasiou, T., Cherian, A., Ross, D., 2004. The Ross II Procedure: Pulmonary Autograft in the Mitral Position. The Annals of Thoracic Surgery 78, 1489-1495.

Baig, K., Punjabi, P., 2008. Heart valve surgery. Surgery (Oxford) 26, 491-495.

Banerjee, R., 2013. Fatigue analysis of arteries using finite element method. M.S. North Dakota State University, United States -- North Dakota.

Bernard, I., 2008. Mitral stenosis still a concern in heart valve diseases. Archives of Cardiovascular Diseases 101, 597-599.

Borazjani, I., 2013. Fluid-structure interaction, immersed boundary-finite element method simulations of bio-prosthetic heart valves. Computer Methods in Applied Mechanics and Engineering 257, 103-116. 
Boronyak, S., Smelko, A., Sacks, M. S., Ramaswamy, S., 2009. Design of a Flow-Stretch-Flexure Bioreactor for Physiologic Conditioning of Engineered Tissue. In Biomedical Engineering Society Annual Fall Meeting, Pittsburgh, PA.

Boubriak, O. A., Urban, J. P. G., Cui, Z. F., 2006. Monitoring of lactate and glucose levels in engineered cartilage construct by microdialysis. Journal of Membrane Science 273, 77-83.

Brown, D. A., MacLellan, W. R., Laks, H., Dunn, J. C., Wu, B. M., Beygui, R. E., 2007. Analysis of oxygen transport in a diffusion-limited model of engineered heart tissue. Biotechnology and Bioengineering 97, 962-975.

Brown, J. W., Ruzmetov, M., Turrentine, M. W., Rodefeld, M. D., 2004. Mitral valve replacement with the pulmonary autograft: Ross II procedure with kabanni modification. Seminars in Thoracic and Cardiovascular Surgery: Pediatric Cardiac Surgery Annual 7, 107-114.

Butcher, J. T., Mahler, G. J., Hockaday, L. A., 2011. Aortic valve disease and treatment: The need for naturally engineered solutions. Advanced Drug Delivery Reviews 63, 242-268.

Cavalcante, J. L., Rodriguez, L. L., Kapadia, S., Tuzcu, E. M., Stewart, W. J., 2012. Role of Echocardiography in Percutaneous Mitral Valve Interventions. JACC: Cardiovascular Imaging 5, 733-746.

Chandran, K. B., Yoganathan, A. P., Rittgers, S. E., 2007. Heart Valves. CRC Press, Atlanta, pp. 333.

Chen, S., Xu, L., Liu, Y., Li, Q., Wang, D., Wang, X., Liu, T., 2013. Donkey Pericardium as an Alternative Bioprosthetic Heart Valve Material. Artificial Organs 37, 248-255.

Chen, G., Wu, Q., 2005. The application of polyhydroxyalkanoates as tissue engineering materials. Biomaterials 26, 6565-6578.

Chen, J., Gutmark, E., Mylavarapu, G., Backeljauw, P. F., Gutmark-Little, I., 2014. Numerical investigation of mass transport through patient-specific deformed aortae. Journal of Biomechanics 47, 544-552.

Chung, S., Vafai, K., 2013. Low-density lipoprotein transport within a multilayered arterial wall-Effect of the atherosclerotic plaque/stenosis. Journal of Biomechanics 46, 574-585. 
Chung, S., Vafai, K., Mechanobiology of low-density lipoprotein transport within an arterial wall-Impact of hyperthermia and coupling effects. Journal of Biomechanics.

Converti, A., Casagrande, M., De Giovanni, M., Rovatti, M., Del Borghi, M., 1996. Evaluation of glucose diffusion coefficient through cell layers for the kinetic study of an immobilized cell bioreactor. Chemical Engineering Science 51, 1023-1026.

Dal Pan, F., Donzella, G., Fucci, C., Schreiber, M., 2005. Structural effects of an innovative surgical technique to repair heart valve defects. Journal of Biomechanics 38, 2460-2471.

Das, D. B., 2007. Multiscale simulation of nutrient transport in hollow fibre membrane bioreactor for growing bone tissue: Sub-cellular scale and beyond. Chemical Engineering Science 62, 3627-3639.

David, T., Hsu, C. H., 1996a. Dynamic analysis and geometry models for the design of bi-leaflet prosthetic mechanical heart valves. Medical Engineering \& Physics 18, 463-476.

David, T., Hsu, C. H., 1996b. The integrated design of mechanical bi-leaflet prosthetic heart valves. Medical Engineering \& Physics 18, 452-462.

Day, P. E., Cleal, J. K., Lofthouse, E. M., Hanson, M. A., Lewis, R. M., 2013. What factors determine placental glucose transfer kinetics? Placenta 34, 953958.

De Bartolo, L., Leindlein, A., Hofmann, D., Bader, A., de Grey, A., Curcio, E., Drioli, E., 2012. Bio-hybrid organs and tissues for patient therapy: A future vision for 2030. Chemical Engineering and Processing: Process Intensification 51, 7987.

de Monte, F., Pontrelli, G., Becker, S., 2013. Chapter 3 - Drug Release in Biological Tissues. Elsevier, Boston, pp. 59-118.

Denny, W. J., Walsh, M. T., Numerical modelling of mass transport in an arterial wall with anisotropic transport properties. Journal of Biomechanics.

Di Martino, E., Pietrabissa, R., Mantero, S., 1997. The computational approach applied to the design and structural verification of a trileaflet polymeric heart valve. Advances in Engineering Software 28, 341-346.

Dohmen, P. M., Lembcke, A., Holinski, S., Pruss, A., Konertz, W., 2011. Ten years of clinical results with a tissue-engineered pulmonary valve. The Annals of Thoracic Surgery 92, 1308-1314. 
Driessen, N. J. B., Mol, A., Bouten, C. V. C., Baaijens, F. P. T., 2007. Modeling the mechanics of tissue-engineered human heart valve leaflets. Journal of Biomechanics 40, 325-334.

El-Hamamsy, I., Chester, A. H., Yacoub, M. H., 2010. Cellular regulation of the structure and function of aortic valves. Journal of Advanced Research 1, 5-12.

Elkayam, U., Bitar, F., 2005. Valvular Heart Disease and Pregnancy: Part II: Prosthetic Valves. Journal of the American College of Cardiology 46, 403-410.

Engelmayr, G. C., Hildebranda, D. K., Sutherland, F. W., Mayer, J. E., Sacksa, M. S., 2003. A novel bioreactor for the dynamic flexural stimulation of tissue engineered heart valve biomaterials. Biomaterials 24, 2523-2532.

Engelmayr, G. C., Soletti, L., Vigmostad, S. C., Budilarto, S. G., Federspiel, W. J., Chandran, K. B., Vorp, D. A., Sacks, M. S., 2008. A Novel Flex-Stretch-Flow Bioreactor for the Study of Engineered Heart Valve Tissue Mechanobiology. Annals of Biomedical Engineering 36, 1-13.

Engelmayr, G. C., Sales, V. L., Mayer, J. E., Sacks, M. S., 2006. Cyclic flexure and laminar flow synergistically accelerate mesenchymal stem cell-mediated engineered tissue formation: Implications for engineered heart valve tissues. Biomaterials 27, 6083-6095.

Ethier, C. R., Moore, J. A., 1997. Oxygen mass transfer calculations in large arteries. Journal of Biomechanics Engineering 119, 469-475.

Fong, P., Shin'oka, T., Lopez-Soler, R. I., Breuer, C., 2006. The use of polymer based scaffolds in tissue-engineered heart valves. Progress in Pediatric Cardiology 21, 193-199.

Frank, B. S., Toth, P. B., Wells, W. K., McFall, C. R., Cromwell, M. L., Hilbert, S. L., Lofland, G. K., Hopkins, R. A., Determining Cell Seeding Dosages for Tissue Engineering Human Pulmonary Valves. Journal of Surgical Research.

Frederick J., S., 2006. New frontiers in the pathology and therapy of heart valve disease: 2006 Society for Cardiovascular Pathology, Distinguished Achievement Award Lecture, United States-Canadian Academy of Pathology, Atlanta, GA, February 12, 2006. Cardiovascular Pathology 15, 271-279.

Gamez, A., Castillo, J. C., Bonilla, J. L., Anguita, M. P., 2011. Infective endocarditis after the Ross procedure. International Journal of Cardiology 147, e53-e54. 
Gandaglia, A., Bagno, A., Naso, F., Spina, M., Gerosa, G., 2011. Cells, scaffolds and bioreactors for tissue-engineered heart valves: a journey from basic concepts to contemporary developmental innovations. European Journal of Cardio-Thoracic Surgery 39, 523-531.

Gao, Z., Xu, C. W., 2011. Glucose metabolism induces mono-ubiquitination of histone H2B in mammalian cells. Biochemical and Biophysical Research Communications 404, 428-433.

Ge, L., Fellow, P., Leo, H. L., Student, P. D., Sotiropoulos, F., Yoganathan, A. P., 2005. Flow in a mechanical bileaflet heart valve at laminar and near-peak systole flow rates: Cfd simulations and experiments. J Biomech Eng 127, 782.

Ge, L., Jones, C., Sotiropoulos, F., Healy, T. M., Yoganathan, A. P., 2003. Numerical simulation of flow in mechanical heart valves: grid resolution and the assumption of flow symmetry. Journal of Biomechanical EngineeringTransactions of the Asme 125, 709-718.

Ghanbari, H., Viatge, H., Kidane, A. G., Burriesci, G., Tavakoli, M., Seifalian, A. M., 2009. Polymeric heart valves: new materials, emerging hopes. Trends in Biotechnology 27, 359-367.

Gill, J. S., Salafia, C. M., Grebenkov, D., Vvedensky, D. D., 2011. Modeling oxygen transport in human placental terminal villi. Journal of Theoretical Biology 291, 33-41.

Gilmanov, A., Sotiropoulos, F., 2005. A hybrid Cartesian/immersed boundary method for simulating flows with 3D, geometrically complex, moving bodies. Journal of Computational Physics 207, 457-492.

Haj-Ali, R., Dasi, L. P., Kim, H., Choi, J., Leo, H. W., Yoganathan, A. P., 2008. Structural simulations of prosthetic tri-leaflet aortic heart valves. Journal of Biomechanics 41, 1510-1519.

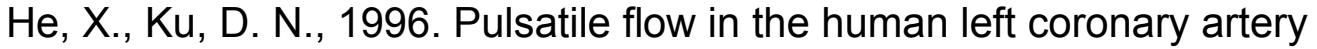
bifurcation: average conditions. Journal of Biomechanics Engineering 118, 74-82.

Hilfiker, A., Kasper, C., Hass, R., Haverich, A., 2011. Mesenchymal stem cells and progenitor cells in connective tissue engineering and regenerative medicine: is there a future for transplantation? Archives of Surgery 396, 489-497.

Hmiel, C. D., 2012. Strain-induced strengthening of bioprosthetic tissue in physiologically super-saturated calcium phosphate solution. M.S. State University of New York at Buffalo, United States -- New York. 
Hoerstrup, S. P., Sodian, R., Daebritz, S., Wang, J., Bacha, E. A., Martin, D. P., Moran, A. M., Guleserian, K. J., Sperling, J. S., Kaushal, S., Vacanti, J., Schoen, F. J., Mayer, J. E., 2000a. Functional Living Trileaflet Heart Valves Grown In Vitro. 102, 44-49.

Hoerstrup, S. P., Sodian, R., Sperling, J. S., Vacanti, J. P., Mayer, J. E., 2000 b. New Pulsatile Bioreactor for In Vitro Formation of Tissue Engineered Heart Valves. Tissue Engineering 6, 75-79.

Hoerstrup, S. P., Zund, G., Schoeberlein, A., Ye, Q., Vogt, P. R., Turina, M. I., 1998. Fluorescence activated cell sorting: a reliable method in tissue engineering of a bioprosthetic heart valve. The Annals of Thoracic Surgery 66, 1653-1657.

Hoerstrup, S. P., Zund, G., Ye, Q., Schoeberlein, A., Schmid, A. C., Turina, M. I., 1999. Tissue engineering of a bioprosthetic heart valve: stimulation of extracellular matrix assessed by hydroxyproline assay. ASAIO Journal (American Society for Artificial Internal Organs : 1992) 45, 397-402.

Hoffman, J. I., 1995. Incidence of congenital heart disease: I. Postnatal incidence. Pediatric Cardiology 16, 103-113.

Hong, T., Choeng-Ryul, C., Chang-Nyung, K., 2009. Characteristics of hemodynamics in a bileafletmechanical heart valve using an implicit FSI method. Engineering and Technology 49, 679-684.

Hose, D. R., Narracott, A. J., Penrose, J. M. T., Baguley, D., Jones, I. P., Lawford, P. V., 2006. Fundamental mechanics of aortic heart valve closure. Journal of Biomechanics 39, 958-967.

Huang, C. Y., Pelaez, D., Dominguez-Bendala, J., Garcia-Godoy, F., Cheung, H. S., 2009. Plasticity of stem cells derived from adult periodontal ligament.

Regenerative Medicine 4, 809-821.

Ishaug-Riley, S. L., Crane-Kruger, G. M., Yaszemski, M. J., Mikos, A. G., 1998. Three-dimensional culture of rat calvarial osteoblasts in porous biodegradable polymers. Biomaterials 19, 1405-1412.

lyengar, S. S., Pontefract, D. E., Barlow, C. W., 2004. Heart valve surgery. Surgery (Oxford) 22, 135-138.

Jacobs, C. R., Yellowley, C. E., Davis, B. R., Zhou, Z., Cimbala, J. M., Donahue, H. J., 1998. Differential effect of steady versus oscillating flow on bone cells. Journal of Biomechanics 31, 969-976. 
Jaggers, J., Harrison, J. K., Bashore, T. M., Davis, R. D., Glower, D. D., Ungerleider, R. M., 1998. The Ross Procedure: Shorter Hospital Stay, Decreased Morbidity, and Cost Effective. The Annals of Thoracic Surgery 65, 1553-1558.

Johnson, A. S., Fisher, R. J., Weir, G. C., Colton, C. K., 2009. Oxygen consumption and diffusion in assemblages of respiring spheres: Performance enhancement of a bioartificial pancreas. Chemical Engineering Science 64, 4470-4487.

Kaazempur-Mofrad, M. R., Wada, S., Myers, J. G., Ethier, C. R., 2005. Mass transport and fluid flow in stenotic arteries: Axisymmetric and asymmetric models. International Journal of Heat and Mass Transfer 48, 4510-4517.

Kadner, A., Hoerstrup, S. P., Zund, G., Eid, K., Maurus, C., Melnitchouk, S., Grunenfelder, J., Turina, M. I., 2002. A new source for cardiovascular tissue engineering: human bone marrow stromal cells. European Journal of CardioThoracic Surgery 21, 1055-1060.

Karim, N., Golz, K., Bader, A., 2006. The Cardiovascular Tissue-Reactor: A Novel Device for the Engineering of Heart Valves. International for Artificial Organs 30, 809-814.

Karner, G., Perktold, K., 2000. Effect of endothelial injury and increased blood pressure on albumin accumulation in the arterial wall: a numerical study. Journal of Biomechanics 33, 709-715.

Khakpour, M., Vafai, K., 2008. Critical assessment of arterial transport models. International Journal of Heat and Mass Transfer 51, 807-822.

Khwaja, S., Nigro, J. J., Starnes, V. A., 2005. The Ross procedure is an ideal aortic valve replacement operation for the teen patient. Seminars in Thoracic and Cardiovascular Surgery: Pediatric Cardiac Surgery Annual 8, 173-175.

Kidane, A. G., Burriesci, G., Edirisinghe, M., Ghanbari, H., Bonhoeffer, P., Seifalian, A. M., 2009. A novel nanocomposite polymer for development of synthetic heart valve leaflets. Acta Biomaterialia 5, 2409-2417.

Kim, D., Choi, H., 2006. Immersed boundary method for flow around an arbitrarily moving body. Journal of Computational Physics 212, 662-680.

King, M., David, T., Fisher, J., 1997. Three-dimensional study of the effect of two leaflet opening angles on the time-dependent flow through a bileaflet mechanical heart valve. Journal of Medical Engineering and Physics 19, 235-241. 
King, M. J., Corden, J., David, T., Fisher, J., 1996. A three-dimensional, timedependent analysis of flow through a bileaflet mechanical heart valve:

Comparison of experimental and numerical results. Journal of Biomechanics 29, 609-618.

Kiris, C., Kwak, D., Rogers, S., Chang, I., 1997. Computational approach for probing the flow through artificial heart devices. Journal of Biomechanical Engineering 119, 452-460.

Kleinstreuer, C., 2006. Biofluid dymamics: Principles and applications. CRC Press, Boca Raton, FL, pp. 472-478.

Koutny, T., 2011a. Estimating reaction delay for glucose level prediction. Medical Hypotheses 77, 1034-1037.

Koutny, T., 2011b. Estimating reaction delay for glucose level prediction. Medical Hypotheses 77, 1034-1037.

Krafczyk, M., Cerrolaza, M., Schulz, M., Rank, E., 1998. Analysis of 3D transient bloodflow passing through and artificial aortic valve by Lattice-Boltzmann methods. Journal of Biomechanics 31, 453-462.

Krafczyk, M., Tolke, J., Rank, E., Schulz, M., 2001. Two-dimensional simulation of fluid-structure interaction using lattice-Boltzmann methods. Computers and Structures 79, 2031-2037.

Krause, E., 1985. Computational fluid dynamics: Its present status and future direction. Computers \& Fluids 13, 239-269.

Kukreja, N., Onuma, Y., Daemen, J., Serruys, P. W., 2008. The future of drugeluting stents. Pharmacological Research 57, 171-180.

Kumar, R., Stepanek, F., Mantalaris, A., 2004. An Oxygen Transport Model for Human Bone Marrow Microcirculation. Food and Bioproducts Processing 82, 105-116.

Lai, Y. G., Przekwas, A. J., 1994. A finite-volume method for fluid flow simulations with moving boundaries. Computational Fluid Dynamics 2, 19-40.

Lantz, J., Karlsson, M., 2012. Large eddy simulation of LDL surface concentration in a subject specific human aorta. Journal of Biomechanics 45, 537-542. 
Le, T. B., Sotiropoulos, F., 2013. Fluid-structure interaction of an aortic heart valve prosthesis driven by an animated anatomic left ventricle. Journal of Computational Physics, 41-62.

Lei, M., Van-Steenhoven, A., Van-Campen, D., 1992. Experimental and numerical analyses of the steady flow field around an aortic Bjork-Shiley standard valve prostheses. Journal of Biomechanics 3, 213-222.

Leong, J., Munnelly, A., Liberio, B., Cochrane, L., Vyavahare, N., 2013. Neomycin and carbodiimide crosslinking as an alternative to glutaraldehyde for enhanced durability of bioprosthetic heart valves. Journal of Biomaterials Applications 27, 948-960.

Leopaldi, A. M., Vismara, R., Lemma, M., Valerio, L., Cervo, M., Mangini, A., Contino, M., Redaelli, A., Antona, C., Fiore, G. B., 2012. In vitro hemodynamics and valve imaging in passive beating hearts. Journal of Biomechanics 45, 11331139.

Leor, J., Amsalem, Y., Cohen, S., 2005. Cells, scaffolds, and molecules for myocardial tissue engineering. Pharmacology \& Therapeutics 105, 151-163.

Li, Y. J., Batra, N. N., You, L., Meier, S. C., Coe, I. A., Yellowley, C. E., Jacobs, C. R., 2004. Oscillatory fluid flow affects human marrow stromal cell proliferation and differentiation. Journal of Orthopaedic Research 22, 1283-1289.

Lichtenberg, A., Breymann, T., Cebotari, S., Haverich, A., 2006. Cell seeded tissue engineered cardiac valves based on allograft and xenograft scaffolds. Progress in Pediatric Cardiology 21, 211-217.

Lim, W. L., Chew, Y. T., Chew, T. C., Low, H. T., 1998. Steady flow dynamics of prosthetic aortic heart valves: a comparative evaluation with PIV techniques. Journal of Biomechanics 31, 411-421.

Liu, X., Fan, Y., Deng, X., Zhan, F., 2011. Effect of non-Newtonian and pulsatile blood flow on mass transport in the human aorta. Journal of Biomechanics 44 , 1123-1131.

Imanov, A., Sotiropoulos, F., 2005. A hybrid Cartesian/immersed boundary method for simulating flows with 3D, geometrically complex, moving bodies. Journal of Computational Physics 207, 457-492.

Lorna, S., 2010. Surgery for valve disease in young people-beyond morbidity and mortality. International Journal of Cardiology 145, 411-412. 
Lotz, J., Meier, C., Leppert, A., Galanski, M., 2002. Cardiovascular Flow Measurement with Phase Contrast MR Imaging: Basic Facts and Implementation. RadioGraphics 22, 651-671.

Martineau, L. C., 2012. Large enhancement of skeletal muscle cell glucose uptake and suppression of hepatocyte glucose-6-phosphatase activity by weak uncouplers of oxidative phosphorylation. Biochimica Et Biophysica Acta (BBA) General Subjects 1820, 133-150.

Martinez, C., Rath, S., Van Gulden, S., Pelaez, D., Alfonso, A., Fernandez, N., Kos, L., Cheung, H., Ramaswamy, S., 2013. Periodontal Ligament Cells Cultured under Steady Flow Environments Demonstrate Potential for Use in Heart Valve Tissue Engineering. Tissue Engineering 19, 458-466.

McQueen, D. M., Peskin, C. S., 1985. Computer-assisted design of butterfly bileaflet valves for the mitral position. Scand J Thorac Cardiovasc Surg 19, 139148.

Mohammadi, H., Mequanint, K., 2011. Prosthetic aortic heart valves: Modeling and design. Medical Engineering \& Physics 33, 131-147.

Mokhbi Soukane, D., Shirazi-Adl, A., Urban, J. P. G., 2007. Computation of coupled diffusion of oxygen, glucose and lactic acid in an intervertebral disc. Journal of Biomechanics 40, 2645-2654.

Munnelly, A. E., Cochrane, L., Leong, J., Vyavahare, N. R., 2012. Porcine vena cava as an alternative to bovine pericardium in bioprosthetic percutaneous heart valves. Biomaterials 33, 1-8.

Neuenschwander, S., P. Hoerstrup, S., 2004. Heart valve tissue engineering. Transplant Immunology 12, 359-365.

Nguyen, M. C., Lim, Y. L., 2007. Benefits of drug-eluting stents in coronary heart disease treatment with emphasis on the diabetic subgroup. Heart \& Lung, Circulation 16, 7-9.

Nobili, M., Morbiducci, U., Ponzini, R., Del Gaudio, C., Balducci, A., Grigioni, M., Maria Montevecchi, F., Redaelli, A., 2008. Numerical simulation of the dynamics of a bileaflet prosthetic heart valve using a fluid-structure interaction approach. Journal of Biomechanics 41, 2539-2550.

Nora, F., Sergio, G., Cristina, R., Constantin, C., Guillaume, S., 2012. FE analysis applied for validation of a biostable aortic valve replacement device: stent and leaflet material selection. International Journal of Biomedical Engineering and Technology 9, 378-394. 
Onate, E., Idelsohn, S. R., Del Pin, F., Aubry, R., 2004. The particle finite element method. An overview. International Journal of Computational Methods, 307.

Oshima, M., Torii, R., Kobayashi, T., Taniguchi, N., Takagi, K., 2001. Finite element simulation of blood flow in the cerebral artery. Computer Methods in Applied Mechanics and Engineering 191, 661-671.

Patnaik, S. S., Wang, B., Weed, B., Wertheim, J. A., Liao, J., 2013.

Decellularized Scaffolds: Concepts, Methodologies, and Applications in Cardiac Tissue Engineering and Whole-Organ Regeneration.

Pedley, T. J., Stephanoff, K. D., 1985. Flow along a channel with a timedependent indentation in one wall: the generation of vorticity waves. Journal of Fluid Mechanics 160, 337-367.

Pelliccioni, O., Cerrolaza, M., Herrera, M., 2007. Lattice Boltzmann dynamic simulation of a mechanical heart valve device. Mathematics and Computers in Simulation 75, 1-14.

Perry, T. E., Kaushal, S., Sutherland, F. W. H., Guleserian, K. J., Bischoff, J., Sacks, M., Mayer, J. E., 2003. Bone marrow as a cell source for tissue engineering heart valves. The Annals of Thoracic Surgery 75, 761-767.

Peskin, C., 1972. Flow patterns around heart valves: a numerical method. Journal of Computational Physics 10, 252-271.

Peskin, C. S., McQueen, D. M., 1980. Modeling prosthetic heart valves for numerical analysis of blood flow in the heart. Journal of Computational Physics $37,113-132$.

Pretorius, V., Jones, A., Taylor, D., Coe, Y., Ross, D. B., 2008. Percutaneous valved stent repair of a failed homograft: Implications for the Ross procedure. Canadian Journal of Cardiology 24, S54-S55.

Prosi, M., Zunino, P., Perktold, K., Quarteroni, A., 2005. Mathematical and numerical models for transfer of low-density lipoproteins through the arterial walls: a new methodology for the model set up with applications to the study of disturbed lumenal flow. Journal of Biomechanics 38, 903-917.

Ramaswamy, S. D., Vigmostad, S. C., Wahle, A., Lai, Y. G., Olszewski, M. E., Braddy, K. C., Brennan, T. M., Rossen, J. D., Sonka, M., Chandran, K. B., 2004. Fluid dynamic analysis in a human left anterior descending coronary artery with arterial motion. Annals of Biomedical Engineering 32, 1628-1641. 
Ramaswamy, S., Boronyak, S., Le, T., Sotiropoulos, F., Holmes, A., Sacks, M. S., 2014. A Novel Bioreactor for Mechanobiological Studies of Engineered Heart Valve Tissue Formation Under Physiological Flow Conditions. Journal of Biomechanical Engineering.

Ramaswamy, S., Boronyak, S., Schmidt, D., Sacks, M. S., 2009a. Design of a Novel Curved Tube Flow-Stretch-Flexure Bioreactor for Mechanistic Studies in Heart Valve Tissue Engineering. In Society for Biomaterials, 2009 Annual Meeting and Exposition, San Antonio, Texas.

Ramaswamy, S., Boronyak, S. M., Engelmayr, G. C., Schmidt, D. E., El-Kurdi, M., Sacks, M. S., 2011. Flow-Stretch-Flexure Bioreactor. 12/959,906.

Ramaswamy, S., Gottlieb, D., Engelmayr Jr., G. C., Aikawa, E., Schmidt, D. E., Gaitan-Leon, D. M., Sales, V. L., Mayer Jr., J. E., Sacks, M. S., 2010. The role of organ level conditioning on the promotion of engineered heart valve tissue development in-vitro using mesenchymal stem cells. Biomaterials 31, 1114-1125.

Ramaswamy, S., Salinas, M., Carrol, R., Landaburo, K., Ryans, X., Crespo, C., Rivero, A., Al-Mousily, F., DeGroff, C., Bleiweis, M., Yamaguchi, H., 2013. Protocol for Relative Hydrodynamic Assesment of tri-leaflet polymer valves. Journal of Visualization Experiments 17, e50335.

Ramaswamy, S., Schmidt, D., Boronyak, S., Sacks, M. S., 2009b. Flow Patterns Under Combined Flexural States for Engineered Heart Valve Tissue Development. In Biomedical Engineering Society Annual Fall Meeting. Pittsburgh, PA.

Ramaswamy, S., Schmidt, D., Kassab, G., 2012. Biomechanics of Heart Valves. Nova Science Publishers, Inc., New York, pp. 175-194.

Ramaswamy, S., Boronyak, S., Sacks, M. S., 2008. Design and Evaluation of a New Flow-Stretch-Flexure Bioreactor for Mechanical Conditioning of Engineered Heart Valve Tissues in the Physiological Range. In The TERMIS-NA 2008 Annual Conference \& Exposition, San Diego, CA.

Rath, S., Salinas, M., Ramaswamy, S., 2014. Marrow Stem Cell differentiation for Valvulogenesis via Oscillatory Flow and Nicotine Agonists: Unusual Suspects? Journal of Long Term Effects of Medical Implants.

Ratner, B. D., Huffman, A. S., Schoen, F. J., Lemons, J. E., An introduction to materials in medicine. pp. 629-637.

Roger, V. L., Go, A. S., Lloyd-Jones, D. M., Adams, R. J., Berry, J. D., Brown, T. M., Carnethon, M. R., Dai, S., de Simone, G., Ford, E. S., Fox, C. S., Fullerton, 
H. J., Gillespie, C., Greenlund, K. J., Hailpern, S. M., Heit, J. A., Ho, P. M., Howard, V. J., Kissela, B. M., Kittner, S. J., Lackland, D. T., Lichtman, J. H., Lisabeth, L. D., Makuc, D. M., Marcus, G. M., Marelli, A., Matchar, D. B., McDermott, M. M., Meigs, J. B., Moy, C. S., Mozaffarian, D., Mussolino, M. E., Nichol, G., Paynter, N. P., Rosamond, W. D., Sorlie, P. D., Stafford, R. S., Turan, T. N., Turner, M. B., Wong, N. D., Wylie-Rosett, J., American Heart Association Statistics Committee and Stroke Statistics Subcommittee, 2011. Heart disease and stroke statistics--2011 update: a report from the American Heart Association. Circulation 123, e18-e209.

Ruel, J., Lachance, G., 2009. A New Bioreactor for the Development of TissueEngineered Heart Valves. Annals of Biomedical Engineering 37, 674-681.

Rus, C., Mesa, D., Concha, M., Casares, J., Suárez de Lezo, J., Delgado, M., Franco, M., Romo, E., Ruiz, M., Vallés, F., 2004. Short-Term Results With the Ross Procedure. Does the Etiology of Aortic Valvulopathy Affect the Outcome? Revista Española De Cardiología (English Edition) 57, 531-537.

Ryan, L. P., Salgo, I. S., Gorman, R. C., Gorman III, J. H., 2006. The Emerging Role of Three-Dimensional Echocardiography in Mitral Valve Repair. Seminars in Thoracic and Cardiovascular Surgery 18, 126-134.

Sacks, M. S., Schoen, F. J., Mayer, J. E., 2009a. Bioengineering Challenges for Heart Valve Tissue Engineering. Annual Review of Biomedical Engineering 11, 289-313.

Sacks, M. S., David Merryman, W., Schmidt, D. E., 2009b. On the biomechanics of heart valve function. Journal of Biomechanics 42, 1804-1824.

Salinas, M., Schmidt, D. E., Libera, M., Lange, R. R., Ramaswamy, S., 2014a. Oscillatory shear stress created by fluid pulsatility versus flexed specimen configurations. Computer Methods in Biomechanics and Biomedical Engineering $17,728-739$.

Salinas, M., Schmidt, D. E., Libera, M., Lange, R. R., Ramaswamy, S., 2014b. Erratum in. Computer Methods in Biomechanics and Biomedical Engineering $17,932$.

Salinas, M., Schmidt, D. E., Libera, M., Lange, R. R., Ramaswamy, S., 2012. Oscillatory shear stress created by fluid pulsatility versus flexed specimen configurations. Computer Methods in Biomechanics and Biomedical Engineering.

Salinas, M., Ramaswamy, S., 2014. Computational simulations predict a key role for oscillatory fluid shear stress in de novo valvular tissue formation. Journal of Biomechanics Accepted. 
Sarioglu, T., Erek, E., Yalçınbas, Y. K., Salihoğlu, E., Sarioglu, A., Tekin, S., 2003. Pericardial collar modification for Ross procedure. Cardiovascular Surgery 11, 229-230.

Schlosshan, D., Aggarwal, G., Mathur, G., Allan, R., Cranney, G., 2011. RealTime 3D Transesophageal Echocardiography for the Evaluation of Rheumatic Mitral Stenosis. JACC: Cardiovascular Imaging 4, 580-588.

Schmidt, D., Mol, A., Odermatt, B., Neuenschwander, S., Breymann, C., Gössi, M., Genoni, M., Zund, G., Hoerstrup, S. P., 2006. Engineering of biologically active living heart valve leaflets using human umbilical cord-derived progenitor cells. Tissue Engineering 12, 3223-3232.

Schmidt, D., Dijkman, P. E., Driessen-Mol, A., Stenger, R., Mariani, C., Puolakka, A., Rissanen, M., Deichmann, T., Odermatt, B., Weber, B., Emmert, M. Y., Zund, G., Baaijens, F. P. T., Hoerstrup, S. P., 2010. Minimally-Invasive Implantation of Living Tissue Engineered Heart Valves: A Comprehensive Approach From Autologous Vascular Cells to Stem Cells. Journal of the American College of Cardiology 56, 510-520.

Schoen, F. J., 2008. Evolving Concepts Of Cardiac Valve Dynamics The Continuum Of Development, Functional Structure, Pathobiology And Tissue Engineering. Circulation 118, 1864-1880.

Sengers, B. G., Van Donkelaar, C. C., Oomens, C. W., Baaijens, F. P., 2005. Computational Study of Culture Conditions and Nutrient Supply in Cartilage Tissue Engineering. Biotechnology Progress 21, 1252-1261.

Sengupta, P. P., Pedrizzetti, G., Kilner, P. J., Kheradvar, A., Ebbers, T., Tonti, G., Fraser, A. G., Narula, J., 2012a. Emerging Trends in CV Flow Visualization. JACC: Cardiovascular Imaging 5, 305-316.

Sengupta, P. P., Pedrizzetti, G., Kilner, P. J., Kheradvar, A., Ebbers, T., Tonti, G., Fraser, A. G., Narula, J., 2012b. Emerging Trends in CV Flow Visualization. JACC: Cardiovascular Imaging 5, 305-316.

Sha, P., 2010. Tricuspid and Pulmonary Valve Disease Evaluation and Management. Revista Española De Cardiología (English Edition) 63, 1349-1365.

Shahbudin H., R., 2010. Choice of Prosthetic Heart Valve in Adults: An Update. Journal of the American College of Cardiology 55, 2413-2426.

Shahriari, S., Maleki, H., Hassan, I., Kadem, L., 2012. Evaluation of shear stress accumulation on blood components in normal and dysfunctional bileaflet 
mechanical heart valves using smoothed particle hydrodynamics. Journal of Biomechanics 45, 2637-2644.

Shim, E., Chang, K., 1994. Three-dimensional vortex flow past a tilting disc valve using a segregated finite element scheme. Computer Dynamics Journal 3, 205.

Siepe, M., Akhyari, P., Lichtenberg, A., Schlensak, C., Beyersdorf, F., 2008. Stem cells used for cardiovascular tissue engineering. European Journal of Cardio-Thoracic Surgery 34, 242-247.

Silverthorn, U., 2005. Human Physiology an Integrated Approach. Pearson/Benjamin Cummings, Michigan, pp. 311-362.

Simon, P., Kasimir, M., Rieder, E., Weigel, G., 2006. Tissue Engineering of heart valves-Immunologic and inflammatory challenges of the allograft scaffold. Progress in Pediatric Cardiology 21, 161-165.

Siu, S. C., Silversides, C. K., 2010. Bicuspid Aortic Valve Disease. Journal of the American College of Cardiology 55, 2789-2800.

Slater, M., Shen, I., Welke, K., Komanapalli, C., Ungerleider, R., 2005. Modification to the Ross procedure to prevent autograft dilatation. Seminars in Thoracic and Cardiovascular Surgery: Pediatric Cardiac Surgery Annual 8, 181184.

Sodian, R., Hoerstrup, S. P., Sperling, J. S., Daebritz, S. H., Martin, D. P., Schoen, F. J., Vacanti, J. P., Mayer, J. E., 2000a. Tissue engineering of heart valves: in vitro experience. Annals of Thoracic Surgery 70, 140-144.

Sodian, R., Hoerstrup, S. P., Sperling, J. S., Daebritz, S., Martin, D. P., Moran, A. M., Kim, B. S., Schoen, F. J., Vacanti, J. P., Mayer, J. E.,Jr, 2000b. Early in vivo experience with tissue-engineered trileaflet heart valves. Circulation 102, III22-9.

Sodian, R., Sperling, J. S., Martin, D. P., Egozy, A., Stock, U., Mayer, J. E.,Jr, Vacanti, J. P., 2000c. Fabrication of a trileaflet heart valve scaffold from a polyhydroxyalkanoate biopolyester for use in tissue engineering. Tissue Engineering 6, 183-188.

Sodian, R., Sperling, J. S., Martin, D. P., Stock, U., Mayer, J. E.,Jr, Vacanti, J. P., 1999. Tissue engineering of a trileaflet heart valve-early in vitro experiences with a combined polymer. Tissue Engineering 5, 489-494.

Sotiropoulos, F., Borazjani, I., 2009. A review of state-of-the-art numerical methods for simulating flow through mechanical heart valves. Medical and Biological Engineering and Computing 47, 245-256. 
Stangeby, D., Ethier, C., 2002. Coupled computational analysis of arterial Idl transport-effects of hypertension. Computer Methods in Biomechanics and Biomedical Engineering 5, 233-241.

Stella, J. A., Liao, J., Sacks, M. S., 2007. Time-dependent biaxial mechanical behavior of the aortic heart valve leaflet. Journal of Biomechanics 40, 3169-3177.

Stelzer, P., 2011. The Ross Procedure: State of the Art 2011. Seminars in Thoracic and Cardiovascular Surgery 23, 115-123.

Storti, M. A., Nigro, N. M., Paz, R. R., Dalcínm, L. D., 2008. Dynamic boundary conditions in computational fluid dynamics. Computer Methods in Applied Mechanics and Engineering 197, 1219-1232.

Sutherland, F. W., Perry, T. E., Yu, Y., Sherwood, M. C., Rabkin, E., Masuda, Y., Garcia, A. G., McLellan, D. L., Engelmayr, G. C., Sacks, M. S., Schoen, F. J., Mayer, J. E., 2005. From Stem Cells to Viable Autologous Semilunar Heart Valve. Circulation, 2783-2791.

Syedain, Z. H., 2009a. Development of a completely biological tissue engineered heart valve. Ph.D. University of Minnesota, United States -- Minnesota.

Syedain, Z. H., 2009b. Development of a completely biological tissue engineered heart valve. Ph.D. University of Minnesota, United States -- Minnesota.

Tai, C. H., Liew, K. M., Zhao, Y., 2007. Numerical simulation of 3D fluid-structure interaction flow using an immersed object method with overlapping grids. Computers \& Structures $85,749-762$.

Talwar, S., Rajesh, M. R., Subramanian, A., Saxena, A., Kumar, A. S., 2005. Mitral valve repair in children with rheumatic heart disease. The Journal of Thoracic and Cardiovascular Surgery 129, 875-879.

Tarbell, J. M., Lever, M. J., Caro, C. G., 1988. The effect of varying albumin concentration of the hydraulic conductivity of the rabbit common carotid artery. Microvascular Research 35, 204-220.

Ungerleider, R. M., Ootaki, Y., Shen, I., Welke, K. F., 2010. Modified Ross Procedure to Prevent Autograft Dilatation. The Annals of Thoracic Surgery 90, 1035-1037.

Vermot, J., Forouhar, A., Liebling, M., Wu, D., Plummer, D., Gharib, M., Fraser, E., 2009. Reversing Blood Flows Act through klf2a to Ensure Normal Valvulogenesis in the Developing Heart. 7, e1000246. 
Vierendeels, J., Dumont, K., Verdonck, P. R., 2008. A partitioned strongly coupled fluid-structure interaction method to model heart valve dynamics. Journal of Computational and Applied Mathematics 215, 602-609.

Vyavahare, P., Chen, P., Joshi, P., Lee, P., Hirsch, P., Levy, P., Schoen, M., Levy, M., 1997. Current Progress in Anticalcif ication for Bioprosthetic and Polymeric Heart Valves. Cardiovascular Pathology 6, 219-229.

Wang, L., Korossis, S., Fisher, J., Ingham, E., Jin, Z., 2011. Prediction of oxygen distribution in aortic valve leaflet considering diffusion and convection. The Journal of Heart Valve Disease 20, 442-448.

Wang, L., Korossis, S., Ingham, E., Fisher, J., Jin, Z., 2008. Computational simulation of oxygen diffusion in aortic valve leaflet for tissue engineering applications. The Journal of Heart Valve Disease 17, 700-709.

Wang, L., Wilshaw, S. P., Korossis, S., Fisher, J., Jin, Z., Ingham, E., 2009. Factors influencing the oxygen consumption rate of aortic valve interstitial cells: application to tissue engineering. Tissue Engineering.Part C, Methods 15, 355363.

Waterbolk, T. W., Hoendermis, E. S., den Hamer, I. J., Ebels, T., 2006.

Pulmonary valve replacement with a mechanical prosthesis: Promising results of 28 procedures in patients with congenital heart disease. European Journal of Cardio-Thoracic Surgery 30, 28-32.

Weber, B., Zeisberger, S. M., Hoerstrup, S. P., 2011a. Prenatally harvested cells for cardiovascular tissue engineering: Fabrication of autologous implants prior to birth. Placenta 32, Supplement 4, S316-S319.

Weber, B., Schoenauer, R., Papadopulos, F., Modregger, P., Peter, S., Stampanoni, M., Mauri, A., Mazza, E., Gorelik, J., Agarkova, I., Frese, L., Breymann, C., Kretschmar, O., Hoerstrup, S. P., 2011b. Engineering of living autologous human umbilical cord cell-based septal occluder membranes using composite PGA-P4HB matrices. Biomaterials 32, 9630-9641.

Westhoff-Bleck, M., Girke, S., Breymann, T., Lotz, J., Pertschy, S., Tutarel, O., Roentgen, P., Bertram, H., Wessel, A., Schieffer, B., Meyer, G. P., 2011. Pulmonary valve replacement in chronic pulmonary regurgitation in adults with congenital heart disease: Impact of preoperative QRS-duration and NT-proBNP levels on postoperative right ventricular function. International Journal of Cardiology 151, 303-306.

Wong, K. K. L., Sun, Z., Tu, J., Worthley, S. G., Mazumdar, J., Abbott, D., 2012. Medical image diagnostics based on computer-aided flow analysis using 
magnetic resonance images. Computerized Medical Imaging and Graphics 36, 527-541.

Yang, N., Vafai, K., 2008. Low-density lipoprotein (LDL) transport in an artery - A simplified analytical solution. International Journal of Heat and Mass Transfer 51, 497-505.

Yang, N., Vafai, K., 2006. Modeling of low-density lipoprotein (LDL) transport in the artery - effects of hypertension. International Journal of Heat and Mass Transfer 49, 850-867.

Yu, P., Lee, T. S., Zeng, Y., Low, H. T., 2009. Fluid dynamics and oxygen transport in a micro-bioreactor with a tissue engineering scaffold. International Journal of Heat and Mass Transfer 52, 316-327.

Zhang, L. T., Gay, M., 2007. Immersed finite element method for fluid-structure interactions. Journal of Fluids and Structures 23, 839-857.

Zilla, P., Brink, J., Human, P., Bezuidenhout, D., 2008. Prosthetic heart valves: Catering for the few. Biomaterials 29, 385-406. 
VITA

MANUEL SALINAS

Born, Ambato, Ecuador

2003-2006

A.A., General Engineering

Broward College

Davie, Florida

2006-2009

B.S., Biomedical Engineering

Florida International University

Miami, Florida

2010

NIH-MBRS-RISE Fellowship (Five years)

2011

Outstanding Masters Student From

College of Engineering and Computing

2009-2011

M.S., Biomedical Engineering

Florida International University

Miami, Florida

2012

NASA Florida Space Grant Consortium Ambassador

2013

Computational Fluid Dynamics

University of Miami

Coral Gables, Florida

2013

Elected AGILE Fellow

2014

Founder - President

Of Computer Simulations Society FIU

2014

Inducted in the Academy of Leaders-FIU

2012-2014

Ph.D., Biomedical Engineering

Florida International University

Miami, Florida 


\section{PUBLICATIONS AND PRESENTATIONS}

Salinas M, Ramaswamy S. Computational Simulations Predict a Key Role for Oscillatory Fluid Shear Stress in De Novo Valvular Tissue Formation. Journal of Biomechanics. Accepted August 2014.

Salinas M, Schmidt DE, Libera M, Lange RR, Ramaswamy S. Oscillatory shear stress created by fluid pulsatility versus flexed specimen configurations. Journal of Computer Methods in Biomechanics and Biomedical Engineering. 2014; 17(7):728-39.

Rath S, Salinas M, Ramaswamy S. Marrow Stem Cell differentiation for Valvulogenesis via Oscillatory Flow and Nicotine Agonists: Unusual Suspects? Journal of Long Term Effects of Medical Implants. (In press) 2014.

Ramaswamy S, Salinas M, Carrol R, Landaburo K, Ryans X, Crespo C, Rivero A, Al-Mousily F, DeGroff C, Bleiweis M, Yamaguchi H: Protocol for Relative Hydrodynamic Assessment of Tri-leaflet Polymer Valves. Journal of Visualized Experiments. 2013; (80)e50335.

Salinas M, Ramaswamy S. Computational Fluid Modeling of Heart Valves. Computational Bioengineering. CRC press. (In press) 2014.

Salinas M, Ramaswamy S. (POSTER) Nutrient Transport During Engineered Heart Valve Tissue Exposure to Steady Flow. Biomedical Engineering Society (BMES) 2014 Annual meeting. San Antonio, TX, October 22-25, 2014.

Salinas M, Ramaswamy S. (POSTER) Oscillatory Fluid-Induced Shear Stresses in Moving Engineered Valvular Tissues. World Congress for Biomechanics. Boston July 6-11, 2014.

Salinas M, Tanet D, Ramaswamy S. (TALK) Mechanical Evaluation of stents for stent graft aortic evaluation. Southern Biomedical Engineering Conference. Miami May1-3, 2013.

Salinas M, Schmidt D, Lange R, Libera M, Ramaswamy S. (POSTER) Computational Prediction of Fluid Induced Stress States in Dynamically Conditioned Engineered Heart Valve Tissues. American Society of Mechanical Engineers (ASME) Summer Bioengineering Conference. Fajardo, Puerto Rico, June 20-23, 2012.

Salinas M, Lange R, Ramaswamy S. (TALK) Heart Valve Tissue Engineering: Implications of Specimen Dynamics. Biomedical and Comparative Immunology Symposium. Miami, FL, March 3-4, 2011. 